\title{
Energy Optimization for Virtualized Network Environments
}

by

Ebrahim Ghazisaeedi, M.Sc.

A thesis submitted to the

Faculty of Graduate and Postdoctoral Affairs

in partial fulfillment of the requirements for the degree of

Doctor of Philosophy in Electrical and Computer Engineering
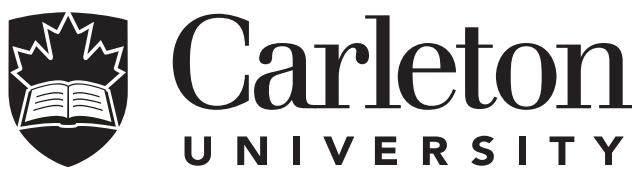

U N I V E R S I T Y

Ottawa-Carleton Institute for Electrical and Computer Engineering

Department of Systems and Computer Engineering

Carleton University

Ottawa, Ontario

August, 2015

(C) Copyright

Ebrahim Ghazisaeedi, 2015 


\section{Abstract}

Information and Communication Technology (ICT) has been estimated to consume $10 \%$ of the total energy consumption in industrial countries. According to the latest measurements, this amount is rapidly increasing by $6 \%$ annually. With the evolved new business model in which Service Providers (SPs) are separated from Infrastructure Providers (InPs), Virtualized Network Environments (VNEs) have been regarded as a promising technology for flexibly utilizing shared communication network resources. VNEs also play a fundamental role toward virtualizing data centers. In this thesis, we suggest different feasible solutions to optimize the energy consumption in a VNE. In this regard, first, we review the corresponding literature in regard to the architecture of a VNE, its performance modelling, several power models, and also existing energy-saving solutions for VNEs. We approach the objective of optimizing the energy consumption in a VNE by defining and solving two main problems. The first problem optimizes the energy consumption in a VNE during the off-peak period. This is feasible by reconfiguring the mapping of already embedded virtual networks for the off-peak time. This is planned in two smaller and simpler sub-problems with increasing the complexity and higher energy-saving levels. Our solutions enable the providers to adjust the level of the reconfiguration and accordingly control the possible traffic disruptions. In the second problem, we propose a novel energy-efficient embedding method that maps heterogeneous MapReduce-based virtual networks onto a heterogeneous data center physical network, energy-wise. We introduce a new incast problem that specifically may happen in Virtualized Data Centers (VDCs). The proposed embedding process also controls the incast queueing delay. 
To my parents 


\section{Acknowledgments}

I would like to express my special appreciation and thanks to my supervisor Professor Changcheng Huang, he has been a tremendous mentor for me. I would like to thank him for encouraging my research and for allowing me to grow as a research scientist. His advice on both research as well as on my career have been priceless.

I would like to thank Professors Pin-Han Ho, Yiqiang Zhao, Richard Yu, and Hussein Mouftah for their careful review and contributions to the thesis.

A special thanks to my family. Words cannot express how grateful I am to my mother, father, and my sister, for all of the sacrifices that they have made on my behalf. Their prayer for me was what sustained me thus far. I would also like to thank all of my friends who supported me in writing, and incented me to strive towards my goal. 


\section{Contents}

Abstract $\quad$ ii

Acknowledgments $\quad$ iv

Table of Contents $\quad$ v

List of Tables viii

List of Figures $\quad$ ix

Acronyms $\quad$ xii

Terms and Definitions $\quad$ xv

1 Introduction $\quad 1$

1.1 Motivation . . . . . . . . . . . . . . . . . . 1

1.2 Objective and Scope . . . . . . . . . . . . . . . . . . 3

1.3 Contributions . . . . . . . . . . . . . . . 5

1.4 Publications . . . . . . . . . . . . . . . . . . 8

1.5 Organization of Thesis . . . . . . . . . . . . . . . . 9 9

2 Literature Review $\quad 10$

2.1 Structure of Virtualized Network Environments . . . . . . . . . . . . 11

2.2 Modelling and Analysis of VNEs . . . . . . . . . . . . . . . . . . 14

2.2.1 VNE Embedding Problems . . . . . . . . . . . . . . . . . . . . 14

2.2.2 Network Performance in VNEs . . . . . . . . . . . . . . . 33

2.2.3 Performance Modelling of Virtualized Servers . . . . . . . . . 42

2.3 Energy-Saving for VNEs . . . . . . . . . . . . . . . . . 45

2.3.1 Node Power Models . . . . . . . . . . . . . . . . . . 46 
2.3.2 Link Power Models . . . . . . . . . . . . . . . . . . . . . . 52

2.3.3 Existing Energy-Saving Solutions for VNEs _ . . . . . . . 57

3 Off-Peak Energy Optimization for Links in a VNE 61

3.1 Introduction . . . . . . . . . . . . . . . . . . 61

3.2 Power Models . . . . . . . . . . . . . . . . . . . . . . . 65

3.3 Integer Linear Programs . . . . . . . . . . . . . . . 66

3.3.1 The Network Model . . . . . . . . . . . . . . . . . . . . . 67

3.3.2 Programs based on Fixed Link Power Model . . . . . . . . . . 68

3.3.3 Programs based on Semi-Proportional Link Power Model . . . 77

3.4 The Heuristic Algorithm . . . . . . . . . . . . . . . . . . . . 78

3.5 Evaluation . . . . . . . . . . . . . . . . . . . . . 81

$3.5 .1 \quad \operatorname{ILPs} \ldots \ldots \ldots \ldots \ldots \ldots$

3.5 .2 The Heuristic . . . . . . . . . . . . . . . . . . . . 86

3.6 Summary . . . . . . . . . . . . . . . . . . . . . 94

4 Off-Peak Energy Optimization for Nodes and Links in a VNE 95

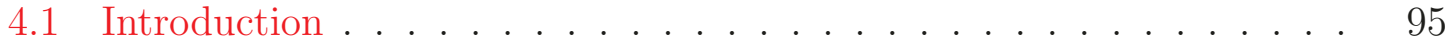

4.2 Power Models . . . . . . . . . . . . . . . . . . . . . . . . . . . . 98

4.3 Integer Linear Programs . . . . . . . . . . . . . . . . . . . . 99

4.3 .1 The Network Model . . . . . . . . . . . . . . . . . . . . . . 101

4.3.2 Programs Based on Fixed Power Model . . . . . . . . . . . 102

4.3.3 Programs Based on Semi-Proportional Power Model . . . . . . 119

4.4 The Heuristic Algorithm . . . . . . . . . . . . . . . . . . . . 120

4.5 Evaluation . . . . . . . . . . . . . . . . . . . . . . . . . 124

$4.5 .1 \quad \operatorname{ILPs} \ldots \ldots \ldots \ldots \ldots \ldots \ldots \ldots$

4.5 .2 The Heuristic . . . . . . . . . . . . . . . . . . . . . . 134

4.6 Summary . . . . . . . . . . . . . . . . . . . . . . . . . . . . . . . 141

5 GreenMap: Green Mapping of Heterogeneous MapReduce-based Virtual Networks onto a Data Center Network and Controlling Incast Queueing Delay 142

5.1 Introduction . . . . . . . . . . . . . . . . . . . . . . . . . 142

5.2 The Network Model . . . . . . . . . . . . . . . . . . . . . . . . . 146

5.3 Power Models . . . . . . . . . . . . . . . . . . . . . . . . . . . . 148 
5.4 The Mixed Integer Disciplined Convex Program . . . . . . . . . . . . 149

5.5 The Heuristic Algorithm . . . . . . . . . . . . . . . . . 157

5.6 Evaluation . . . . . . . . . . . . . . . . . . . . . . . 169

5.6 .1 The MIDCP . . . . . . . . . . . . . . . . 171

5.6 .2 The Heuristic . . . . . . . . . . . . . . . . . . . . . 175

5.7 Summary . . . . . . . . . . . . . . . . . 180

6 Conclusion and Future Works 181

6.1 Off-Peak Energy Optimization for a VNE . . . . . . . . . . . . . . . . 181

6.2 GreenMap . . . . . . . . . . . . . . . . . . 183

List of References $\quad 185$ 


\section{List of Tables}

2.1 Node and link attributes in VNEs . . . . . . . . . . . . 20

2.2 Parameters of physical link power consumption . . . . . . . . 53

4.1 Re-allocation combinations for virtual nodes of $l_{n}^{a_{m}, b_{m}}$, and the required off-peak traffic to be rerouted . . . . . . . . . . . . 107 


\section{List of Figures}

2.1 A Virtualized Network Environment . . . . . . . . . . . . . 11

2.2 A VNE embedding process . . . . . . . . . . . . . . . . . . 12

$2.3 \mathrm{M} / \mathrm{M} / 1$ queue model for non-virtualized severs . . . . . . . . . . . . 42

2.4 Queue model for virtual servers $[1] \ldots \ldots \ldots$

2.5 Two dimensional Markov-Chain model for two virtualized severs on a single physical server $[1] \ldots \ldots \ldots$. . . . . . . . . . . . . 4 45

2.6 Three types of power models . . . . . . . . . . . . . . . 47

3.1 Example: An embedded virtual link onto a substrate network . . . 68

3.2 Example: Generated random topologies . . . . . . . . . . . . . 82

3.3 Off-peak link energy optimization by global link reconfiguration vs. local link reconfiguration (splittable traffic) . . . . . . . . . . 83

3.4 (a) The total link power consumption of off-peak link energy optimization by global link reconfiguration. (b) The total link power consumption of off-peak link energy optimization by local link reconfiguration for splittable traffic. (c) The total link power consumption of off-peak link energy optimization by local link reconfiguration for non-splittable traffic . . . . . . . . . . . . . . . . . .

3.5 The total saved power with off-peak link energy optimization by local link reconfiguration for splittable and non-splittable traffic . . . . . 86

3.6 The BILP vs. the heuristic for off-peak link energy optimization by local link reconfiguration (non-splittable traffic) . . . . . . . 87

3.7 The total link power consumption of the heuristic for off-peak link energy optimization by local link reconfiguration . . . . . . . . 88

3.8 The link reconfiguration heuristic for the different numbers of involved

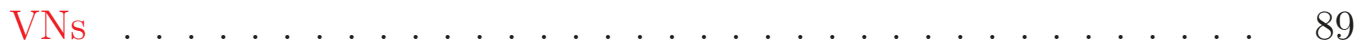

3.9 The effect of changing the stress rate threshold on the heuristic's outcome 90

3.10 The link reconfiguration heuristic vs. our previous algorithm . . . . 91 
3.11 The mean link utilization over different configurations . . . . . . . . . 91

3.12 The total link power consumption before and after applying the heuristic on the GÉANT simulation setup . . . . . . . . . . . . . . . . . . 92

3.13 The run time for different methods . . . . . . . . . . . . . . . 93

4.1 The total node and link power consumption based on the off-peak ratio by the MILPs for ONL-LNLs-F and ONL-LNLs-SP . . . . . . . . . . 127

4.2 The total saved power in the network, the total saved power in physical nodes, and the total saved power in physical links based on the off-peak ratio by the MILP for ONL-LNLs-F . . . . . . . . . . . . . . . . . . . 128

4.3 The total node and link power consumption based on the off-peak ratio by the MILPs for ONL-LLs-F and ONL-LLs-SP . . . . . . . . . . . . 128

4.4 The total node and link power consumption based on the off-peak ratio by the BILPs for ONL-LLns-F and ONL-LLns-SP . . . . . . . . . . . 129

4.5 The total saved power in physical nodes and links based on the off-peak ratio by the MILPs for ONL-LNLs-F and ONL-LLs-F . . . . . . . . .

4.6 The total saved power in physical nodes and links based on the off-peak ratio by the ILPs for ONL-LLs-F and ONL-LLns-F . . . . . . . . . . 131

4.7 The total saved power in physical nodes and links based on the off-peak ratio by the MILPs for ONL-LLs-F and ONL-LLs-SP . . . . . . . . . 131

4.8 The total saved power in physical nodes and links based on the number of involved virtual networks by the MILP for ONL-LLs-F . . . . . . .

4.9 The total saved power in physical nodes and links based on the number of virtual nodes per VN by the MILP for ONL-LLs-F . . . . . . . . . 133

4.10 The total node and link power consumption based on the off-peak ratio before and after applying the heuristic with different values of $K$, for ONL-LLns-F . . . . . . . . . . . . . . . . . . . . . 135

4.11 The total saved power in physical nodes and links based on the offpeak ratio by the BILP and the heuristic with different values of $K$, for ONL-LLns-F . . . . . . . . . . . . . . . . . .

4.12 The total saved power in physical nodes and links based on the number of involved virtual networks by the heuristic with $K=5$, for ONLLLns-F . . . . . . . . . . . . . . . . . . . . . .

4.13 The total saved power in physical nodes and links based on the number of virtual nodes per VN by the heuristic with $K=5$ for ONL-LLns-F 
4.14 The impact of different $\tilde{s_{2}}$ thresholds on the total saved power in physical nodes and links by the heuristic when $K=5$ for ONL-LLns-F . . 138

4.15 Comparison between the suggested heuristic in this chapter and previously proposed heuristic in Chapter 3 . . . . . . . . . . . . . . . . . 139

4.16 The mean link utilization based on the off-peak ratio over different

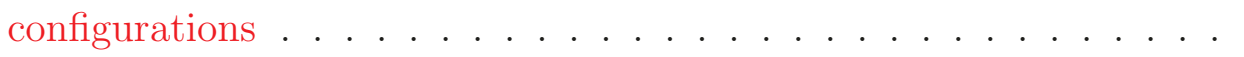

4.17 Required run time based on the different number of virtual nodes per VN, for the BILP and the heuristic with $K=5$, for ONL-LLns-F . . 140

5.1 Example: A MapReduce-based VN's topology . . . . . . . . . . . . . 147

5.2 The heuristic's decision making process . . . . . . . . . . . . 163

5.3 Topology of the data center network in a small simulation setup . . . 169

5.4 The total power consumption based on different numbers of virtual nodes per VN for the state-of-the-art algorithm, the MIDCP for GreenMap, and the heuristic for GreenMap . . . . . . . . . . . . . .

5.5 The mean incast queueing delay based on different CPU demands of a computation-based virtual node for the MIDCP, as well as the chosen maximum tolerable queueing delay $\hat{D}$ for a virtual link . . . . . . . . 173

5.6 The total VDC's power consumption based on different CPU demands of a computation-based virtual node for the MIDCP, and the MIDCP when the incast constraints are relaxed . . . . . . . . . . . . . . . . . 174

5.7 The total VDC's power consumption based on different numbers of virtual nodes per VN for the state-of-the-art algorithm, and the heuristic for GreenMap . . . . . . . . . . . . . . . . . . . . . .

5.8 The mean inast queuing delay based on different numbers of virtual nodes per VN for the heuristic for GreenMap, as well as the maximum tolerable queuing delay $\hat{D}$ for a virtual link . . . . . . . . . . . . . . . 177

5.9 The mean acceptance ratio based on different values of $\hat{D}$ for the heuristic for GreenMap . . . . . . . . . . . . . . . . . . 178

5.10 The mean acceptance ratio based on different mean traffic rates of a mapper virtual node for the heuristic for GreenMap . . . . . . . . . .

5.11 The mean incast queuing delay based on different mean traffic rates of a mapper virtual node for the heuristic for GreenMap . . . . . . . . . 179 


\section{Acronyms}

$\begin{array}{ll}\text { BILP } & \text { Binary Integer Linear Program } \\ \text { BT } & \text { British Telecom } \\ \text { CPU } & \text { Central Processing Unit } \\ \text { GeSI } & \text { Global e-Sustainability Initiative } \\ \text { ICT } & \text { Information and Communication Technology } \\ \text { IP } & \text { Internet Protocol } \\ \text { ISP } & \text { Internet Service Provider } \\ \text { InP } & \text { Infrastructure Provider } \\ \text { ILP } & \text { Integer Linear Program } \\ \text { LSP } & \text { Label-Switched Path } \\ \text { MPLS } & \text { Multi Protocol Label Switching } \\ \text { MTU } & \text { Maximum Transmission Unit } \\ \text { MIB } & \text { Management Information Base } \\ \text { MIDCP } & \text { Mixed Integer Disciplined Convex Program } \\ \text { MMPP } & \text { Markov Modulated Poisson Process } \\ \text { MILP } & \text { Mixed Integer Linear Program } \\ \text { NF } & \text { Non-functional } \\ \text { ON } & \text { Overlay Networks } \\ \text { OL-GLs-F } & \text { Off-peak Link energy optimization by Global Link reconfiguration, } \\ & \text { Splittable traffic, Fixed power model }\end{array}$


OL-GLs-SP Off-peak Link energy optimization by Global Link reconfiguration, Splittable traffic, Semi-Proportional power model

OL-LLs-F Off-peak Link energy optimization by Local Link reconfiguration, Splittable traffic, Fixed power model

OL-LLs-SP Off-peak Link energy optimization by Local Link reconfiguration, Splittable traffic, Semi-Proportional power model

OL-LLns-F Off-peak Link energy optimization by Local Link reconfiguration, Non-Splittable traffic, Fixed power model

OL-LLns-SP Off-peak Link energy optimization by Local Link reconfiguration, Non-Splittable traffic, Semi-Proportional power model

ONL-LNLs-F Off-peak Node and Link energy optimization by Local Node and Link reconfiguration, Splittable traffic, Fixed power model

ONL-LNLs-SP Off-peak Node and Link energy optimization by Local Node and Link reconfiguration, Splittable traffic, Semi-Proportional power model

ONL-LLs-F Off-peak Node and Link energy optimization by Local Link reconfiguration, Splittable traffic, Fixed power model

ONL-LLs-SP Off-peak Node and Link energy optimization by Local Link reconfiguration, Splittable traffic, Semi-Proportional power model

ONL-LLns-F Off-peak Node and Link energy optimization by Local Link reconfiguration, Non-Splittable traffic, Fixed power model

ONL-LLns-SP Off-peak Node and Link energy optimization by Local Link reconfiguration, Non-Splittable traffic, Semi-Proportional power model

QoS Quality of Service

RTT Round Trip Time

RTO Retransmission Timeout

SP Service Provider

SLO Service Level Objective

SLA Service Level Agreement

SDN Software-defined Networking

TCP Transmission Control Protocol

ToR Top-of-Rack 


$\begin{array}{ll}\text { UDP } & \text { User Datagram Protocol } \\ \text { VDC } & \text { Virtualized Data Center } \\ \text { VNE } & \text { Virtualized Network Environment } \\ \text { VN } & \text { Virtual Network } \\ \text { VM } & \text { Virtual Machine } \\ \text { VLAN } & \text { Virtual Local Area Network } \\ \text { VPN } & \text { Virtual Private Network } \\ \text { WDM } & \text { Wavelength-Division Multiplexing }\end{array}$




\section{Terms and Definitions}

\begin{tabular}{|c|c|}
\hline$A()$. & A node/link's attribute \\
\hline$\check{A}()$. & Attribute of an allocated virtual resource \\
\hline$\alpha()$. & Status of a node/link \\
\hline$\alpha(.,)$. & $\begin{array}{l}\text { A binary variable denotes whether a virtual node is allocated in a sub- } \\
\text { strate node or not }\end{array}$ \\
\hline$\hat{\alpha}()$. & A node/link's status before embedding \\
\hline$\check{\alpha}()$. & A node/link's status after embedding \\
\hline$\dot{b}()$. & Blocking probability \\
\hline$\beta()$. & Status of a traffic capacity, after reconfiguration \\
\hline$B$ & A large number \\
\hline$\grave{c}()$. & Cost \\
\hline$\grave{c}_{c}()$. & Congestion cost \\
\hline$C()$. & Capacity \\
\hline$\hat{C}()$. & Requested capacity \\
\hline$\hat{C}_{b}()$. & Requested bandwidth capacity \\
\hline$\hat{C}_{c}()$. & Requested processing capacity \\
\hline$\hat{C}_{c}^{\check{m}}()$. & $\begin{array}{l}\text { Minimum amount of required CPU capacity per allocated physical ma- } \\
\text { chine for a computation-based virtual node }\end{array}$ \\
\hline$\hat{C}_{c}^{\hat{m}}()$. & $\begin{array}{l}\text { Maximum amount of required CPU capacity per allocated physical ma- } \\
\text { chine for a computation-based virtual node }\end{array}$ \\
\hline$\check{C}()$. & Allocated capacity \\
\hline
\end{tabular}




$\begin{array}{ll}\dot{C}_{c}(.) & \text { Off-peak processing demand } \\ \check{C}_{b}^{i, j}(.) & \begin{array}{l}\text { Allocated traffic capacity in } l_{s}^{i, j} \text { to a virtual link, or bundled allocated } \\ \text { traffic capacity in } l_{s}^{, j j}\end{array} \\ \check{C}_{c}(.) & \text { Allocated processing capacity } \\ \breve{C}_{b}(.) & \text { Available bandwidth capacity } \\ \breve{C}_{c}(.) & \text { Available processing capacity } \\ C_{b}(.) & \text { Bandwidth capacity } \\ C_{c}(.) & \text { CPU capacity } \\ C_{i}(., .) & \text { Minimum incast processing capacity } \\ d & \text { Delay/Latency } \\ d_{p}(.) & \text { Propagation delay } \\ d_{q}(.) & \text { Queuing delay } \\ \hat{D}^{i, j} & \text { Maximum tolerable queuing delay in a virtual link } \\ d_{n}^{i, j}(m) & \text { An intermediate optimization variable } \\ \dot{d}_{n}^{x, y}(m) & \text { Fraction of } \hat{C}_{b}\left(l_{n}^{a^{m}, b^{m}}\right) \text { that needs to be allocated to } l_{n}^{x, y}(m) \\ \ddot{d}^{i, j}\left(l_{n}^{x, y}(m)\right) & \text { Amount of allocated traffic capacity to } l_{n}^{x, y}(m) \text { in } l_{s}^{i, j} \\ \Delta^{i, j} & \text { Dissimilarity function of all the attributes between } i \text { th node and } j \text { th node } \\ \Delta_{m}^{i, j} & \text { Dissimilarity of } m \text { th attribute between } i \text { th node and } j \text { th node } \\ E_{s} & \text { Set of edges in a substrate network graph } \\ E_{n} & \text { Set of edges in } n \text {th VN graph } \\ E_{v}^{m} & \text { Set of edges of the involved VN with the largest number of virtual links } \\ \eta(.) & \text { Number of involved VNs in a substrate node/link } \\ E_{n}(t) & \text { Departed traffic load from a service component by time } t \\ f_{n}^{i, j}(m) & \text { Set of all the involved VNs } \\ & \text { Reapacity in } l_{s}^{i, j} \text { to } l_{n}^{a,}, b_{m} \text { (may be part of link recon- }\end{array}$


$f_{n}^{\prime i, j}(m) \quad$ Re-allocated traffic capacity in $l_{s}^{i, j}$ to $l_{n}^{a_{m}, b_{m}}$ (part of node reconfiguration sub-problem)

$f^{i, j}\left(\check{C}_{b}^{x, y}\right) \quad$ Re-allocated traffic capacity in $l_{s}^{i, j}$ to $\check{C}_{b}^{x, y}$

$\phi(.,$.$) \quad Status of an allocated virtual node/link in a substrate node/link$

$\check{\phi}(.,$.$) \quad Fraction of processing/switching demand of a virtual node that is allo-$ cated in a substrate node

$\tilde{\phi}(.,$.$) \quad Re-allocation status of an allocated virtual node/link in a substrate$ node/link

$\check{\phi}($.$) \quad Total allocated processing capacity to virtual nodes in a substrate node$

$G_{s} \quad$ Substrate network graph

$G_{n} \quad n$th virtual network graph

$g f($.$) \quad General objective function to be defined based on the traffic classes$

Hessian matrix

$H_{t} \quad$ Header of Transport layer

$H_{i} \quad$ Header of IP layer

$\hbar($.$) \quad Traffic scalability factor$

$\kappa_{m}^{i, j} \quad$ Priority coefficients of $m$ th attribute between $i$ th node and $j$ th node

$K \quad$ Number of shortest paths

$L_{s} \quad$ Total number of substrate links

$l_{s}^{i, j} \quad$ Substrate link that connects $i$ th substrate node to $j$ th substrate node

$L_{n} \quad$ Total number of virtual links in $n$th VN

$l_{n}^{a, b} \quad$ Virtual link belonging to $n$th virtual network, connects $a$ th virtual node to $b$ th virtual node

$l_{n}^{a_{m}, b_{m}} \quad m$ th virtual link in $n$th virtual network, connects the virtual node mapped onto $a_{m}$ th substrate node to the virtual node mapped onto $b_{m}$ th substrate node

$l_{n}^{x, y}(m) \quad$ A sub virtual link of $l_{n}^{a^{m}, b^{m}}$ that connects the allocated source virtual node in $v_{s}^{x}$ to the allocated sink virtual node in $v_{s}^{y}$

$\ddot{L}($.) $\quad$ Set of substrate link candidates 


\begin{tabular}{|c|c|}
\hline$l()$. & Loss rate \\
\hline$\check{L}\left(l_{s}^{i, j}, t\right)$ & Number of virtual links allocated in $l_{s}^{i, j}$ at time $t$ \\
\hline$\underline{L}$ & Number of physical links in the found longest substrate path \\
\hline$\lambda$ & Arrival mean rate \\
\hline$\lambda_{n}^{\mathcal{M}}$ & Summation of arrival rates of all mappers in $n$th VN \\
\hline$\tilde{\lambda}$ & Waxman parameter \\
\hline$\breve{L}()$. & Set of adjacent links \\
\hline$L(t)$ & Traffic load function \\
\hline$\mu$ & Service mean rate \\
\hline$\tilde{\mu}$ & Waxman parameter \\
\hline$M$ & The largest virtual link bandwidth demand in $\Phi$ \\
\hline$N_{s}$ & Total number of substrate nodes \\
\hline$N_{n}$ & Total number of virtual nodes in $n$th VN \\
\hline$\ddot{N}()$. & Set of substrate node candidates \\
\hline$\check{N}\left(v_{s}^{i}, t\right)$ & Number of virtual nodes allocated in $v_{s}^{i}$ at time $t$ \\
\hline$O_{o}$ & Overhead of overlay networks \\
\hline$O_{e}$ & Overhead of Ethernet \\
\hline$O_{i}$ & Overhead of IP layer \\
\hline$O_{t}$ & Overhead of Transport layer \\
\hline$O_{T}$ & Total overheads \\
\hline$\omega_{n}^{m}$ & $\begin{array}{l}\text { A binary variable to show the status of intermediate substrate nodes over } \\
\text { the allocated substrate path to } l_{n}^{a_{m}, b_{m}}\end{array}$ \\
\hline$\dot{\omega}_{n}^{m}$ & $\begin{array}{l}\text { A binary variable to show the reconfiguration status of the relay nodes } \\
\text { of } l_{n}^{a_{m}, b_{m}}\end{array}$ \\
\hline$\ddot{\omega}_{n}^{m}$ & $\begin{array}{l}\text { A binary variable to show the reconfiguration status of the source and } \\
\text { sink nodes of } l_{n}^{a_{m}, b_{m}}\end{array}$ \\
\hline$p_{s}^{i, j}$ & Substrate path that connects $i$ th substrate node to $j$ th substrate node \\
\hline
\end{tabular}




\begin{tabular}{|c|c|}
\hline$p_{s}^{T}$ & Set of all possible paths in a substrate network \\
\hline$p_{s}^{\prime}$ & Sub-set of substrate paths \\
\hline$\check{p}_{n}^{a, b}$ & Substrate path which $l_{n}^{a, b}$ is mapped onto \\
\hline$\psi$ & Tuneable weight \\
\hline$P(j)$ & Probability of being in state $j$ \\
\hline$\tilde{p}()$. & Power consumption of a link or node \\
\hline$\tilde{p}^{b}()$. & Base power consumption \\
\hline$\tilde{p}^{m}()$. & Maximum power consumption \\
\hline$\tilde{p}^{c}()$. & Power consumption of the unit $\mathrm{CPU}$ utilization in the unit time \\
\hline$\tilde{p}^{e}()$. & Power consumption of a packet processor engine \\
\hline$q_{l}()$. & Queue length \\
\hline$\grave{r}()$. & Revenue \\
\hline$\grave{r}_{u}$ & Revenue earned per carried call per unit time \\
\hline$r()$. & Traffic rate \\
\hline $\bar{r}$ & Mean traffic rate \\
\hline$r_{n}^{m}$ & Off-peak traffic demand of $l_{n}^{a_{m}, b_{m}}$ \\
\hline$r_{n}^{i, j}(m)$ & Off-peak traffic demand of $l_{n}^{a_{m}, b_{m}}$ in $l_{s}^{i, j}$ \\
\hline$\hat{r}_{b}^{i, j}$ & Bundled off-peak traffic demand in $l_{s}^{i, j}$ \\
\hline$R T T_{L}$ & Longest RTT \\
\hline$\rho$ & Traffic intensity \\
\hline$R(t)$ & Arrival traffic load to the service component by time $t$ \\
\hline$\tilde{s}()$. & Stress rate \\
\hline$s_{p}$ & Packet Size \\
\hline$s_{l}$ & Payload size \\
\hline$S(t)$ & Traffic capability function \\
\hline
\end{tabular}




\begin{tabular}{|c|c|}
\hline$S_{i}(t)$ & $i$ th traffic capability function \\
\hline$S_{e}(t)$ & End-to-end traffic capability function \\
\hline$S_{-} L$ & Sorted list of nodes/links \\
\hline$\sigma$ & Standard deviation \\
\hline $\mathcal{T}$ & Stress rate's treshold \\
\hline$U()$. & Node/Link utilization \\
\hline$U_{c}()$. & CPU utilization \\
\hline$\Upsilon^{i, j}$ & $j$ th port of $i$ th router \\
\hline$\Upsilon$ & Set of router ports corresponding to the considered LSP \\
\hline$\left|\Upsilon^{i}\right|$ & Number of line cards in $i$ th router \\
\hline$V_{s}$ & Set of vertices in a substrate network graph \\
\hline $\bar{V}_{s}$ & Set of server nodes in a substrate network \\
\hline$\tilde{V}_{s}$ & Set of switch/router substrate nodes in a substrate network \\
\hline$V_{s}^{\prime}$ & Sub-set of substrate nodes \\
\hline$v_{s}^{i}$ & $i$ th substrate node \\
\hline $\bar{v}_{s}^{i}$ & $i$ th substrate node that is a server \\
\hline$\tilde{v}_{s}^{i}$ & $i$ th substrate node that is a switch/router \\
\hline$V_{n}$ & Set of vertices in $n$th VN graph \\
\hline$\dot{V}_{n}$ & Set of mapper virtual nodes in $n$th VN \\
\hline$\ddot{V}_{n}$ & Set of reducer virtual nodes in $n$th $\mathrm{VN}$ \\
\hline$\tilde{V}_{n}$ & Set of shuffler virtual nodes in $n$th VN \\
\hline$v_{n}^{k}$ & $k$ th virtual node in $n$th $\mathrm{VN}$ \\
\hline$\dot{v}_{n}^{k}$ & $k$ th virtual node that is a mapper, in $n$th $\mathrm{VN}$ \\
\hline$\ddot{v}_{n}^{k}$ & $k$ th virtual node that is a reducer, in $n$th $\mathrm{VN}$ \\
\hline$\tilde{v}_{n}^{k}$ & $k$ th virtual node that is a shuffler, in $n$th $\mathrm{VN}$ \\
\hline
\end{tabular}




\begin{tabular}{|c|c|}
\hline $\mathcal{V}_{n}^{k}(i)$ & Removable amount of allocated processing capacity to $\ddot{v}_{n}^{k}$ in $\bar{v}_{s}^{i}$ \\
\hline $\mathcal{V}_{n}^{\prime k}$ & Total removable amount of allocated processing capacity to $\ddot{v}_{n}^{k}$ \\
\hline $\mathcal{V}_{n}^{* k}$ & Total removed processing capacity for $\ddot{v}_{n}^{k}$ \\
\hline$\varrho_{n}^{m}\left(v_{s}^{i}\right)$ & Status of $v_{s}^{i}$ over the allocated substrate path to $l_{n}^{a_{m}, b_{m}}$ \\
\hline$\varrho_{n}^{\prime m}\left(v_{s}^{i}\right)$ & $\begin{array}{l}\text { Status of } v_{s}^{i} \text {, that is a relay node, over the allocated substrate path to } \\
l_{n}^{a_{m}, b_{m}}\end{array}$ \\
\hline$W()$. & Weight \\
\hline $\bar{x}$ & An average result \\
\hline$\zeta$ & Base power consumption ratio \\
\hline$z^{i, j}()$. & Status of a re-allocated commodity in $l_{s}^{i, j}$ \\
\hline$z_{n}^{i, j}(m)$ & Re-allocation status of $l_{n}^{a_{m}, b_{m}}$ in $l_{s}^{i, j}$ \\
\hline
\end{tabular}




\section{Chapter 1}

\section{Introduction}

\subsection{Motivation}

Nowadays, Information and Communication Technology (ICT) plays a fundamental role in everyday life of individuals. It is difficult to imagine a world without the infrastructure that connects people and transfers their information across the globe. Significant advantages of communication networks have stimulated the demand for this technology. It is predicted that the size of the Internet network doubles every 5.32 years [2]. The increase in population's demand, spreading of broadband access and the new services offered by ICT, have triggered the warnings about the energy consumption of the communication technology [3]. The ICT's energy consumption is important to the world due to two main reasons [4]:

1. The environmental reason. It is needed to reduce wastes for controlling CO2 emission.

2. The cost reason. The operators would like to provide services with the minimum cost. However, the cost of energy is high, so by decreasing the energy consumption they can pay less for the energy.

Several reports from different ICT organizations over the world confirm the increasing demand of energy in this technology, which is a concern. In the case that no green technology would be deployed in communication networks, Global eSustainability Initiative (GeSI) predicts 35.8TWh energy consumption for European telecom operators in 2020, while they have already consumed 21.4TWh in 2010 [5]. The European Commission DG INFSO has the similar estimation and predicts an 
energy consumption of 35.8TWh for ICT in 2020 if no green network technologies would be used [6]. Besides, Deutsche Telekom in [7] stated 2\% increase in the total energy consumption from year 2006 to 2007, due to the technology developments, increasing transmission volumes and network expansions. In addition, British Telecom (BT) reported 2.5TWh energy consumption for its networks over the 2008 financial year [8]. This happens while the ICT related devices, in 2007, have consumed about $10 \%$ of the United Kingdom's energy consumption [9]. This problem is even worse when we consider servers and data center clusters. The volume of data needed to be processed by servers in data centers is increasing every day [10], and consequently the energy consumption of their infrastructure is growing. The data centers are estimated to consume $1.4 \%$ of the worldwide electricity energy consumption, while this grows with the rate of $12 \%$ per year [11]. These infrastructures in USA have consumed 61 billion KWh for a cost of 4.5 billion dollars in 2006 [12]. In Amazon's data centers, $53 \%$ of the total budget, over 15 years period, has been used for the operation of servers, while $42 \%$ of the total budget has been considered for the energy-related costs. This includes $19 \%$ of the direct energy consumption and $23 \%$ for the cooling infrastructure [13]. All the cited reports show the growing energy consumption in ICT, which confirms the necessity of implementing energy-saving mechanisms in this technology. Consequently, it is vital to study and design effective methods that not only control the rapidly increasing energy consumption, but also save the energy.

Recently, virtualization has been proposed to share resources in a network environment [14]. A Virtualized Network Environment (VNE) supports the coexistence of multiple Virtual Networks (VNs) over a single physical network [15]. A VNE embedding process maps virtual nodes and links onto physical nodes and paths, respectively. A VNE uses the actual resources more efficiently by sharing a physical network's capacity among multiple virtual networks. Each virtual network is isolated from others, and might run its desired network protocols and services. Network virtualization decouples the functionality of the current networks' architecture into Infrastructure Providers (InPs) and Service Providers (SPs). Besides, Virtual Machines (VMs) traditionally virtualize physical servers' resources. VNs together with the VMs underpin Virtualized Data Centers (VDCs). Traditional data centers are moving toward virtualized data centers in order to address cloud computing limitations regarding the network performance, security, and manageability [16]. Hence, network virtualization has been regarded as a promising technology to flexibly share 
the resources, and therefore the corresponding solutions to energy-saving in this type of network become essential.

However, as far as energy efficiency is concerned, few research works have considered energy-saving in virtualized network environments. A VNE needs distinct energy-saving solutions that are designed specially for its architecture. In a VNE embedding process, it is required to map both virtual nodes and virtual links onto the substrate network. This is more complex than non-virtualized networks in which nodes are fixed. Besides, virtual network demands do not necessarily mean the same as current demands [17]. VN demands are the capacities that the virtual network customers ask from the SPs. They are the upper bounds of the demands generated, processed, and transported by a VN. Hence, in an allocated virtual network, further energy optimization can be carried out applying any of the existing energy-saving techniques [17]. Moreover, allocated virtual networks might have different quality of service requirements, so it is not possible to treat all of them in a similar way. For instance, imagine two virtual links that are mapped onto a physical link. The first virtual link is a part of a virtual network that is throughput-sensitive. However, the second virtual link belongs to another virtual network that is delay-sensitive. It is not efficient to treat all the traffic in the physical link similarly.

\subsection{Objective and Scope}

According to the discussion in Section 1.1, the main objective of this thesis is to develop new energy-saving solutions for a virtualized network environment. Multiple fundamental steps are required to be taken toward this objective.

First, we study the architecture of a VNE to give a clear understanding of its evolved structure. It also helps to discover any possible opportunities to save the energy in such an environment. We also review different studies in the literature in regard to modelling a VNE embedding process, and analyzing its performance. Accurate knowledge of a VNE's architecture and its processes, prepares us to discover energy-saving opportunities in this environment. The main energy consumers in a VNE are physical network elements. Power models of different physical nodes and links, as of the two most important physical network elements, are reviewed. The power models determine the factors affect the energy consumption of the network elements, so we can save the energy more effectively by targeting them. A VNE does 
not change physical power models provided for non-virtualized networks, because the a substrate network is still the main energy consumer in its architecture. Nonetheless, mapping virtual networks onto a physical network might increases the substrate network's energy consumption. As far as energy efficiency is concerned, very few research works have considered energy-saving in virtualized network environments. In this regard, the literature is surveyed regarding existing energy-saving solutions for VNEs. We study their advantages and disadvantages. We also discuss the open research areas.

According to the open research areas, we plan to approach the objective by defining and solving two main problems. The first problem optimizes the energy consumption in a VNE during the off-peak time, by reconfiguring the mapping of embedded VNs once networks go from the peak time to the off-peak time. This problem is planned in two smaller and simpler sub-problems with increasing the complexity and higher energy-saving levels.

Due to the simpler technical implementation and the high potential of energysaving in network links, the first sub-problem in the first problem is restricted to energy-saving solutions for links in a VNE. It reconfigures the mapping of virtual links according to the off-peak traffic demands. We formulate multiple novel energy-saving reconfiguration methods that globally/locally optimize the link energy consumption in VNE during the off-peak time. The proposed fine-grained local reconfiguration enables the providers to adjust the level of the reconfiguration, and accordingly control the possible traffic disruptions. An Integer Linear Program (ILP) is formulated for each solution according to two power models, and considering the impact of traffic splittability. Because the formulated ILPs are not scalable to large network sizes, a novel heuristic algorithm is also suggested. The proposed solutions have been verified through extensive simulations.

Because physical nodes are also essential energy consumers in VNEs, in the second sub-problem of the first problem, we discuss multiple energy-saving solutions that locally optimize the node and link energy consumption in a VNE, during the offpeak period, by reconfiguring the mapping of already allocated virtual nodes and links. The proposed reconfigurations enable the providers to adjust the level of the reconfiguration, and accordingly control the possible traffic disruptions. An ILP is formulated for each solution, according to two power models, and considering the impact of traffic splittability. Because the defined ILPs are $\mathcal{N} \mathcal{P}$-hard, a novel heuristic 
algorithm is also suggested. The proposed energy-saving methods are evaluated over random VNEs.

In the second problem, we propose GreenMap, a novel energy-efficient embedding method that maps heterogeneous MapReduce-based virtual networks onto a heterogeneous data center network. Besides, we introduce a new incast problem that specially may happen in VDCs. GreenMap also controls the incast queueing delay. We formulate a Mixed Integer Disciplined Convex Program (MIDCP) for this method. Because the formulated MIDCP is $\mathcal{N} \mathcal{P}$-hard, we also propose a novel and scalable heuristic for GreenMap. We evaluate GreenMap through extensive simulations.

\subsection{Contributions}

The contributions of this thesis are:

1. We propose different methods for reconfiguring the mapping of virtual links during off-peak time, to optimize the off-peak energy consumption of links in a VNE.

(a) We propose global and local optimization programs in form of ILPs for this problem.

(b) The solutions are formulated according to two power models.

(c) A coarse-grained and a fine-grained reconfiguration method are suggested for this problem.

(d) We discuss how differently we should approach the problem in the case of non-splittable traffic in comparison to splittable traffic, to have a wide enough search zone for re-mapping.

(e) We also present a heuristic reconfiguration algorithm that could achieve closely to the optimum results, but much faster than the optimization solution.

(f) Our methods do not decrease the network admittance ratio for new virtual networks.

(g) We define a stress rate for a substrate link. So, our solutions enable the providers to change the level of the reconfiguration by adjusting the stress rate's threshold, and therefore control the possible interruptions. 
(h) Our solutions are not limited to a sub-topology.

(i) We evaluate the proposed solutions by extensive simulations, and study the impact of different factors.

(j) The simulation results prove that the significant improvement in saving power by our method in comparison to the state-of-the-art.

(k) To the best of our knowledge, there is not such a comprehensive study in the literature that considers simultaneously a global/local, coarse-grained/fine-grained energy-efficient reconfiguration of a VNE for splittable/non-splittable traffic, according to two power models.

2. We suggest multiple solutions that formulate unique off-peak energy-saving reconfiguration strategies for nodes and links in a VNE.

(a) We propose a local optimization program in form of an MILP that reconfigures the mapping of both virtual nodes and link during the off-peak time, to optimize the total energy consumption of nodes and links in a $\mathrm{VNE}$ in that period.

(b) We propose local optimization programs in form of ILPs that reconfigure the mapping of virtual links during the off-peak time, to optimize the energy consumption of intermediate substrate nodes and substrate links in a VNE in that period.

(c) The solutions are formulated according to two power models.

(d) Different programs are formulated for splittable and non-splittable traffic in order to study the impact of traffic splittability.

(e) We present a scalable heuristic algorithm that can achieve closely to the optimum results, but considerably faster than the optimization program.

(f) Our methods do not decrease the network admittance ratio for new virtual networks.

(g) We define different stress rates for distinct types of substrate nodes. Therefore, different from any related research studies, the proposed methods enable the providers to control the level of reconfiguration and possible traffic interruptions.

(h) Our solutions are not limited to a sub-topology, and therefore they have a larger degree of freedom to save the energy. 
(i) We assess the impact of different parameters on the energy-saving capability of the discussed solutions, through extensive simulations.

(j) To the best of our knowledge, these problems are not defined and formulated mathematically in any published studies.

3. We also propose GreenMap, a novel energy-efficient embedding method that maps heterogeneous MapReduce-based virtual networks onto a heterogeneous data center network.

(a) GreenMap is formulated as a MIDCP.

(b) A novel and scalable heuristic is also proposed for the problem that can achieve closely to the optimum points.

(c) Our approach makes it probable to split and map computation-based virtual nodes onto a data center network. Accordingly, it enables the providers to embed computation-based VNs onto a data center network.

(d) GreenMap handles heterogeneity of MapReduce-based VNs and a data center network.

(e) For the first time, we introduce a new incast problem for virtualized data centers.

(f) We demonstrate a novel approach that controls the introduced incast queueing delay.

(g) We tackle the incast problem during the provisioning process. Therefore, it prevents the incast problem from happening at the first place.

(h) We examine both the MIDCP and the heuristic through extensive simulations, and check impacts of different factors.

(i) We demonstrate how controlling the incast queueing delay may affect the energy-saving level and the networks' admittance ratio.

(j) To the best of our knowledge, this is the first study on the energy-efficient embedding of MapReduce-based virtual networks onto a data center network. 


\subsection{Publications}

The sections of this thesis appeared in the following publications:

1. Ebrahim Ghazisaeedi, Changcheng Huang, and James Yan, "Off-Peak EnergyWise Link Reconfiguration for Virtualized Network Environment," In 14th International Symposium on Integrated Network and Service Management (IM), IFIP/IEEE, pp. 814-817, Ottawa, 11-15 May 2015.

2. Ebrahim Ghazisaeedi, and Changcheng Huang, "Off-Peak Energy Optimization for Links in Virtualized Network Environment," Transactions on Cloud Computing, IEEE, PP, no. 99, (2015).

3. Ebrahim Ghazisaeedi, and Changcheng Huang, "Energy-Efficient Virtual Link Reconfiguration for Off-Peak Time," Accepted for Global Communications Conference (GLOBECOM): Selected Areas in Communications: Green Communications and Computing, IEEE, San Diego, CA, 6-10 December 2015.

4. Ebrahim Ghazisaeedi, and Changcheng Huang, "Energy-Aware Node and Link Reconfiguration for Virtualized Network Environments," Accepted for Computer Networks Journal, Special Issue on Communications and Networking in the Cloud II, Elsevier, (2015).

5. Ebrahim Ghazisaeedi, and Changcheng Huang, "EnergyMap: Energy-Efficient Embedding of MapReduce-based Virtual Networks and Controlling Incast Queueing Delay," Under Review for 15th Network Operations and Management Symposium (NOMS 2016), IEEE/IFIP, Istanbul, 25-29 April 2016.

6. Ebrahim Ghazisaeedi, and Changcheng Huang, "GreenMap: Green Mapping of Heterogeneous MapReduce-based Virtual Networks onto a Data Center and Managing Incast Queueing Delay," Under Review for Journal on Selected Areas in Communications: Series on Green Communications and Networking (Issue 2), IEEE, (2015). 


\subsection{Organization of Thesis}

The remainder of this thesis is organized as follow: Chapter 2 reviews the literature in three major sub-sections. First, the structure of a VNE and its different processes are discussed in Section 2.1. Second, modelling and analysis of a VNE are studied in Section 2.2. Third, Section 2.3 reviews node and link power models, as well as the very recent energy-saving researches for VNEs. The problem of off-peak energy optimization for links in a VNE is described and multiple solutions are proposed in Chapter 3. Off-peak energy optimization problem for nodes and links in a VNE is defined and different methods are suggested in Chapter 4. GreenMap is formulated and solved in Section 5. Finally, the thesis concludes and recommends the future works in Chapter 6. 


\section{Chapter 2}

\section{Literature Review}

According to Section 1.1, it is vital to develop new energy-saving mechanisms for virtualized network environments. In order to come up with effective solutions, we study the corresponding literature in this chapter.

Recently, VNEs have been proposed and attracted researchers' attention. A virtualized network environment allows coexistence of multiple virtual networks on a single physical network. In this environment, virtual networks are isolated from each other and they might run different services and protocols. On one hand, researchers have studied and analyzed VNEs in different points of view such as a VNE' architecture, VNE embedding processes, etc. On the other hand, many energy-saving mechanisms for different types of communications networks are proposed. Nevertheless, VNEs and energy-saving techniques have been studied separately, and there are only very few initial works that concerned about energy efficiency in a VNE.

In this chapter, we review the architecture of a VNE in Section 2.1 to give a clear picture of its structure. Section 2.2 surveys the literature in regard to modelling and analysis of VNEs. It reviews VNE embedding processes, which map requested virtual networks onto a substrate network. Besides, this section studies the network performance in VNEs, and analyzes the performance in virtualized servers. Section 2.3 reviews the energy-saving problem specifically for VNEs. In this section, we discuss the sources of energy consumption in a VNE, and describe different power models. In addition, it surveys existing energy-saving solutions for VNEs, their advantages and disadvantages. 


\subsection{Structure of Virtualized Network Environ- ments}

Communication networks and specifically the Internet have became very popular. This popularity is the biggest impediment to their further growth. Because of a multiprovider architecture of the Internet, modifications to its design is restricted to simple updates over network technologies and protocols $[14,18]$. Network virtualization has been proposed as an innovation in the networking to bring a dynamic and flexible structure in which design modifications are simply possible.

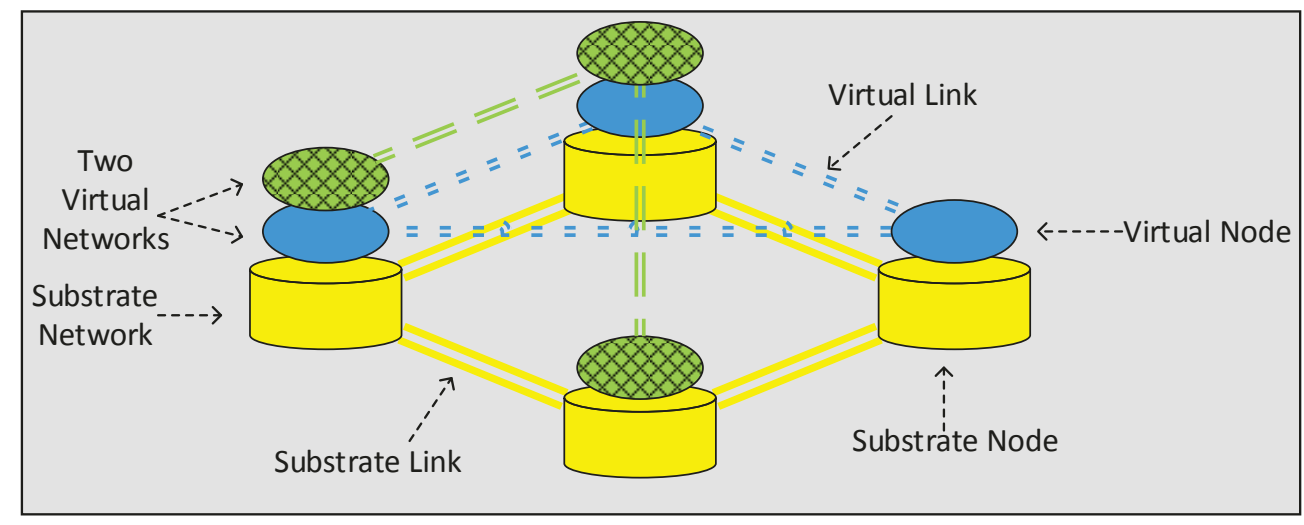

Figure 2.1: A Virtualized Network Environment

In the case that a networking environment supports coexistence of multiple virtual networks over a same physical network, it is called a virtualized network environment [15]. This is shown in Figure 2.1. Virtualization can be achieved by logical separation and segmentation of nodes and also links. A node can be a server, a switch, or a router. Hence, as a customer point of view, there is a dedicated network for each customer while they can run their desired network architecture, protocol, and services. Every single virtual network consists of virtual nodes and links that are mapped onto an underlay physical network. This makes a virtualized network environment.

Virtualized network environments cover different technologies. It is possible to categorize virtual networks in four different types of technologies. Firstly, Virtual Local Area Networks (VLANs), which contain multiple hosts with a common domain. VLANs are normally considered as a layer 2 (Data Link) architecture [19]. Secondly, Virtual Private Networks (VPNs) are the other technology which is covered in VNEs. A VPN has been implemented over different layers. A layer 3 VPN [20,21] uses 
Internet Protocol (IP) or Multi Protocol Label Switching (MPLS), as its network layer protocol. A layer 2 VPN [22,23] provides an end-to-end connection using Ethernet, ATM or Frame Relay layer 2 protocols, and there is also Layer 1 VPNs. Thirdly, active and programmable networks are considered as an instance of network virtualization [15]. The programmable networks separate communication hardware from the control plane [24]. Finally, overlay network creates a virtual topology over a physical topology. This type of network is implemented in the application layer. Overlay networks are very popular and used widely due to their applications.

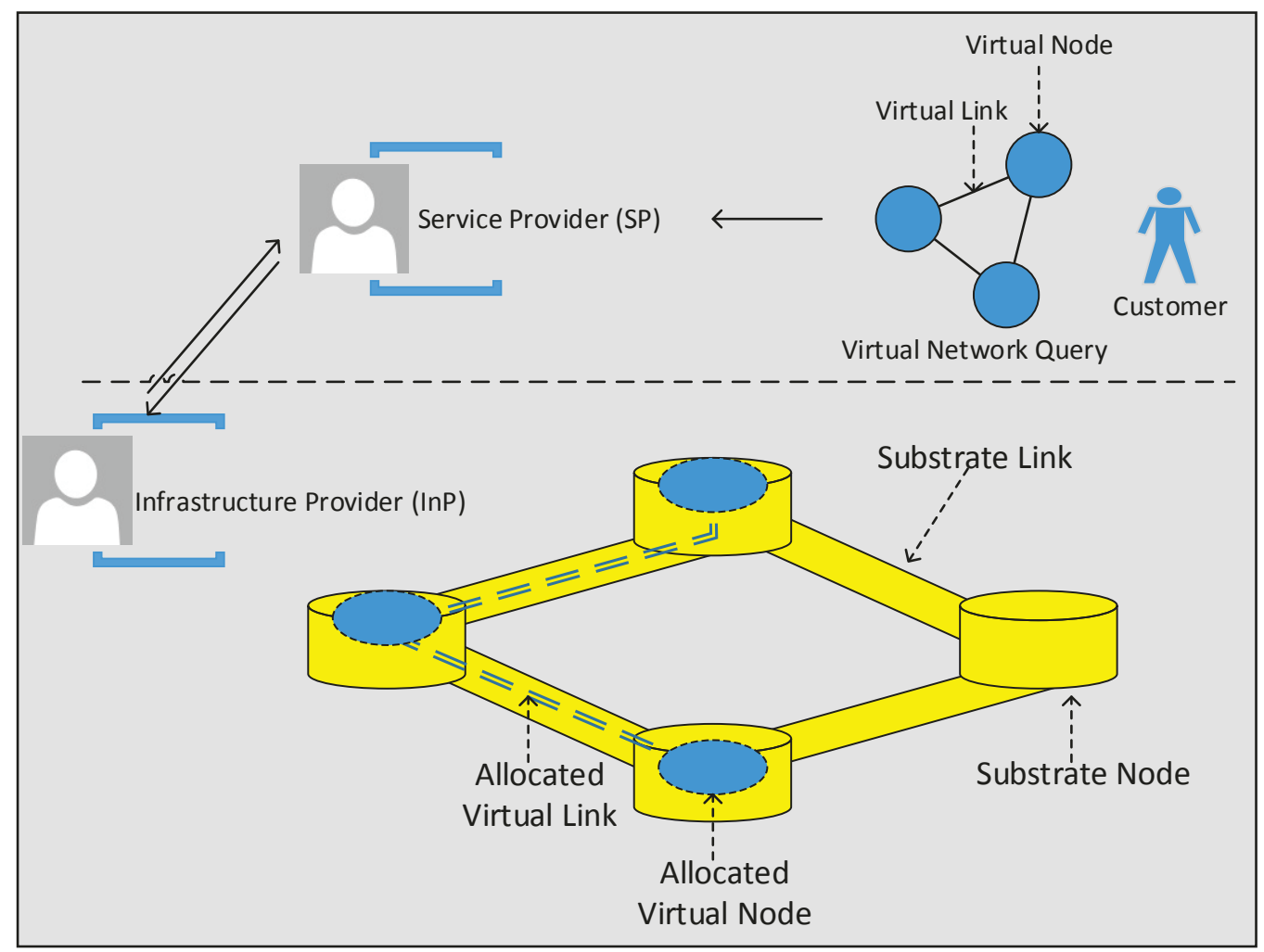

Figure 2.2: A VNE embedding process

VNEs propose decoupling of functionalities by dividing current Internet Service Providers (ISPs) into Infrastructure Providers (InPs), Service Providers (SPs), and Virtual Network Users $[14,25,26]$. These terms are described in the followings:

- Infrastructure Provider: This provider owns the underlay physical substrate network. It needs to discover its actual physical resources to offer for virtualization. 
- Service Provider: The service provider is responsible for assigning and allocating physical resources to virtual network demands.

- Virtual Network User: The VN user request a VN demand from a service provider. The VN User can be an end-host, a service provider, or a virtual network provider [27].

In a virtualized network environment, as it is shown in Figure 2.2, a VN user sends a request to a service provider and asks to map a requested virtual network onto a physical substrate. The VN request may include different demands like the minimum Central Processing Unit (CPU) rate and the memory for each virtual node, the minimum bandwidth and the maximum tolerable delay for each virtual link [28]. Afterwards, the infrastructure provider assists the service provider to assign the requested virtual nodes and links in a specific set of virtual resources extracted from the actual physical resources [27]. The embedding process occurs in three steps as follow:

1. Candidate discovery or matching: The infrastructure provider prepares and sends its actual resources and also virtual resources to the service provider. Then service provider tries to find a set of candidates from nodes and links which match the VN query of the VN user. The resources can be all the links and their bandwidth capacity, all the nodes and their CPU capacity, the memory, ports, and etc. There are several different techniques in the literature, which help infrastructure providers to make sure they have discovered all their available resources and also have the updated network devices' status.

2. Candidate selection: In the second step, the service provider tries to choose the best selection of virtual set from found candidates in the first step. In this case, the best set is defined based on the InP, the SP and the VN user' constraints. Different algorithms according to different objectives and constraints have been proposed in the literature in this regard.

3. Candidate binding: In the final step, the found virtual resources will be allocated/reserved to the VN user [27].

After the embedding process, allocated virtual networks run their own protocols and services, regardless of other active virtual networks. Note that VNE embedding processes are also called VNE mapping processes, or provisioning processes. 


\subsection{Modelling and Analysis of VNEs}

In VNEs, one of the most important challenges is the embedding or mapping problem. Section 2.2.1 goes through this problem. For this problem, we model a substrate network, a virtual network, and a mapping process. Multiple VNE embedding methods are proposed in several papers [28-35]. We study their suggested embedding processes according to their defined objectives, and review their advantages and disadvantages.

Besides, the virtual network customers and also InPs have specific needs of network performance which has to be satisfied by the service providers. Consequently, network performance metrics in VNEs are essential to be studied. The end-to-end service offered by a virtual network may pass through multiple InPs over a substrate network. Therefore, it is necessary to model the end-to-end service delivery in a VNE and study its performance dependence to each of the service components. This subject is reviewed in 2.2.2. Two most important network performance metrics are throughput and delay. Throughput and delay for VNEs are also surveyed in 2.2.2.

In addition, servers are one of the essential network elements in the network virtualization. Performance models for virtualized servers are discussed by benefiting from the queuing theory in Section 2.2.3. Both non-virtualized and virtualized servers are modelled in order to make the differences between them more clear.

\subsubsection{VNE Embedding Problems}

As it is discussed, the role of ISPs has been decoupled into two entities in VNEs, infrastructure providers and service providers. Infrastructure providers manage a physical substrate network and offer physical network resources, while service providers afford the end-to-end services. When a customer needs a virtual network, it sends its virtual network request to a service provider. Then, the SP makes a candidate list and chooses the best possible mapping for that specific query, according to the available physical resources and information received from the InP and the customer. Here is where the challenge of virtual network embedding comes up. The problem statement is clear: How should services providers map a virtual network query onto the physical resources in a substrate network, respect to the virtual and substrate networks' constraints?

In order to analyze a VNE embedding problem, it is required to formulate three

models. First, it is necessary to have a model for a substrate network, which describes 
the actual physical network's topology and its constraints. The model has to include all the physical constraints and concerns of infrastructure providers. Second, there should be a model for every virtual network, which reveals the requested virtual network's topology and all the customer's requirements. Finally, we need a model for the embedding process. The embedding model describes the mapping of virtual queries onto a physical substrate network, according to the defined objectives and constraints. In this section, we discuss each of the substrate network model, virtual network model, and the embedding model.

Several embedding techniques have been proposed for VNEs [28-35], according to different embedding objectives. A common VNE embedding objective is minimizing the embedding cost, or maximizing the embedding revenue. Different research studies define various embedding costs or revenues. Other embedding objectives are also defined by some studies. We also review some of the existing VNE embedding techniques and their defined objectives, in this section.

\section{Substrate Network Models}

In the majority of papers [28-32,36] that discuss a VNE embedding problem, a substrate network $G_{s}$ is modelled by an undirected/directed graph $G_{s}=\left(V_{s}, E_{s}\right)$ including different metrics. Where $V_{s}$ stands for the substrate graph's vertices, and $E_{s}$ denotes its edges. Vertices express nodes and edges refer to links. $N_{s}=\left|V_{s}\right|$ is the total number of substrate nodes in $G_{s}$, and $L_{s}=\left|E_{s}\right|$ is the total number of substrate links in $G_{s}$. We might use an undirected graph when all the links in the respective substrate network are symmetric. So, if there is a substrate link $l_{s}^{i, j}$ that connects the $i$ th physical node to the $j$ th physical node with a specific amount of bandwidth capacity, there is also a substrate link $l_{s}^{j, i}$ that connects the $j$ th physical node to the $i$ th physical node with the same amount of bandwidth capacity. However, when the substrate network is not symmetric, and therefore the metrics are not the same in both directions, we might use a directed graph. Here is where the graph theory comes up and all its prosperities can be helpful. The difference between the substrate models in different papers is the metrics which are added to the graph in order to fully express a substrate network's constraints. We review some of them here.

The authors in [29] added two other parameters to a substrate network model. They modelled a substrate network with an undirected graph $G_{s}=$ 
$\left(V_{s}, E_{s}, A\left(V_{s}\right), A\left(E_{s}\right)\right)$. The first two parameters stand for nodes and links of a substrate network. $A\left(V_{s}\right)$ and $A\left(E_{s}\right)$ demonstrate node attributes and link attributes, respectively. The authors in [29] considered the CPU capacity and location for node attributes, and a link attribute denotes a physical link's bandwidth capacity. They also have a parameter that expresses the set of all loopless paths in a substrate network. Choosing the CPU capacity as the most important attribute of substrate nodes is reasonable, because a portion of a physical node's CPU capacity will be assigned to a virtual node and so it is required to express the available CPU capacity amount in the model. Here, a substrate node is a server or a router. The location is also important, as the majority of VN queries coming from customers specify the location of their virtual nodes; this can be a specific city or country. Furthermore, a substrate link has a limited size of bandwidth capacity and each of the virtual networks requests a specific amount of bandwidth in their virtual links. Therefore, the most important attribute for a physical link is the bandwidth capacity.

Ines Houidi and others in [30] represented a substrate network with a weighted undirected graph. They categorized a substrate network's attributes in functional and non-functional ones. The functional attributes state the properties and characteristics of the substrate resources as well as their static metrics like the node type, the node processor type and the capacity, or the link type, the network interface type and its location, cost, etc. The node type can be switch or router, and the node capacity can be defined for CPU and the node network processor. The link type shows the link virtualization technology and the layer which is virtualized, like VLAN, VPN, or L3/L2. However, the non-functional attributes in [30] are defined for dynamic parameters like the available node CPU capacity, the available node bandwidth capacity, actual Quality of Service (QoS) parameters and Geographic coordinates. Although adding functional information like the node type, may help to fully express the actual network, it might make a complicated model which is hard to analyze. We need to have enough information in the model to fully express the network, as while as keeping the model simple enough to analyze.

Jing Lu and Jonathan Turner in [31] also used an undirected graph to model their substrate network. Nonetheless, the edges in their graph are associated with their length. The length is used to express the shortest path distance between each pair of nodes. They also added the location to their graph's nodes to reveal their access nodes in which traffic enters and leaves the substrate network. Besides, their 
substrate network model has an attribute for describing the virtual traffic in form of a set of general traffic constraints. Each of the constraints is simply an upper bound on the allowed traffic [31]. The location and the link length are important when we are discussing routing algorithms and traffic engineering in the network virtualization. The physical node's location in regard to finding the access/edge nodes is vital, when we have more than one underlay substrate network supporting a single end-to-end service. The dependency of a virtual end-to-end service to each of the underlay substrate networks will be discussed in Section 2.2.2.

Yong Zhu and Mostafa Ammar in [32] also modelled a substrate network with an undirected graph. However, based on their requirements they added time stamps for the links and nodes to use their lifetime in their algorithm. Lifetime may be used to avoid a network section to get overloaded, while there is an idle section. The load balancing is considered as the main objective of embedding algorithms in some studies.

The authors in [28] presented a substrate physical network by a weighted undirected graph. The weight for physical nodes shows the available capacity of the node, while the weight for the links expresses two non-negative values. It includes an additive weight value which denotes the cost and delay of a substrate link, and also another value which denotes the available bandwidth capacity associated to a physical link. They derived the bandwidth capacity $C_{b}\left(p_{s}^{i, j}\right)$ for a substrate path $p_{s}^{i, j}$ that connects the $i$ th substrate node to the $j$ th substrate node, by finding the minimal bandwidth in the substrate links $l_{s}^{x, y}$ along the substrate path. This is calculated in Equation 2.1. They also measured the weight $W\left(p_{s}^{i, j}\right)$ (it can be related to cost, delay, or jitter) for a substrate path $p_{s}^{i, j}$, by finding sum of the weights $W\left(l_{s}^{x, y}\right)$ for the substrate links $l_{s}^{x, y}$ along the substrate path $p_{s}^{i, j}$. This is calculated in Equation 2.2.

$$
\begin{aligned}
& C_{b}\left(p_{s}^{i, j}\right)=\min _{(x, y) \in p_{s}^{i, j}} C_{b}\left(l_{s}^{x, y}\right) \\
& W\left(p_{s}^{i, j}\right)=\sum_{(x, y) \in p_{s}^{i, j}} W\left(l_{s}^{x, y}\right)
\end{aligned}
$$

In a virtual network embedding process, a customer asks a specific amount of bandwidth capacity for a virtual link. However, an SP may map this virtual link query onto a substrate path, and not only a substrate link. Therefore, it is needed to measure the minimal bandwidth along a substrate path. The bandwidth over a path 
is equal to the minimum bandwidth associated with any link over the path, which is the bandwidth bottleneck. $C_{b}\left(p_{s}^{i, j}\right)$ measures this metric. Nonetheless, in order to calculate the other performance metrics like delay or cost, it is required to sum all the delays or costs the packet takes and pays to travel over the path. $W\left(p_{s}^{i, j}\right)$ performs the same job.

[28] has a wider view in terms of the attributes used to express a substrate network. It is a good idea to calculate the available bandwidth and delay for all the possible paths in a substrate network in order to respond fast to the queries needed to be mapped to a path. Besides, it includes the delay of the substrate links, that is an advantage. This is an important metric, in the case we have specific tolerable latency for the virtual link queries.

\section{Virtual Network Models}

Similar to the substrate network models, the $n$th virtual network is also modelled by an undirected/directed graph $G_{n}=\left(V_{n}, E_{n}\right)$ in several research papers [28-32,36], where $V_{n}$ stands for the set of vertices of the $n$th virtual network graph, and $E_{n}$ denotes the set of edges of the $n$th virtual network graph. Vertices and edges represent nodes and links, respectively. $N_{n}=\left|V_{n}\right|$ is the total number of virtual nodes in $G_{n}$ and $L_{n}=\left|E_{n}\right|$ is the total number of virtual links in $G_{n}$. We know the allocated virtual network graph is a sub-graph of the substrate network graph, as it is a virtual network mapped onto the physical substrate network. In the case the requested virtual links are residual, and the requested bandwidth between two virtual nodes is the same in both directions, the undirected graphs can be used to model the virtual network topology and its attributes. Therefore, if there is a virtual link $l_{n}^{a, b}$ belonging to the $n$th $\mathrm{VN}$, that connects the $a$ th virtual node to the $b$ th virtual node with a specific amount of bandwidth capacity, then there is another virtual link $l_{n}^{b, a}$ that connects the $b$ th virtual node to the ath virtual node with the same amount of bandwidth capacity. Nonetheless, if the requested bandwidth is different over the virtual links, then it is needed to use directed graphs in order to mathematically express the required virtual network. Of course, considering undirected graphs makes it easier to analyze a network. It is desirable to add some metrics to a virtual network request model in order to express a customer's requirements for each of the virtual nodes and links. Different papers considered different metrics to express the customer's queries. The common requirement between all these papers is the minimum required CPU capacity 
for virtual nodes, and the minimum required bandwidth capacity for virtual links.

The authors in [29] presented a virtual network request by an undirected graph $G_{n}=\left(V_{n}, E_{n}, A\left(V_{n}\right), A\left(E_{n}\right)\right)$. Their virtual network request normally contains node and link constrains which are defined based on the substrate network metrics and constraints. The CPU capacity and the location constraints for virtual nodes and the bandwidth capacity constraint for virtual links of the $n$th VN, are shown with $A\left(V_{n}\right)$ and $A\left(E_{n}\right)$, respectively. When we are talking about the constraints coming from the virtual network side, they are setting the minimum requested attributes. This is different to what is discussed in regard to substrate networks, while they express the available resources.

Besides, Ines houidi in [30] also modelled a virtual network query, requested by a customer and sent to a service provider, with a weighted undirected graph. Their virtual network model, similar to their substrate network model, is associated with the minimum processor capacity as well as the type and the location for virtual nodes. Their virtual links in the weighted undirected graph is associated with the minimum required bandwidth capacity for each virtual link as well as the link type and the required quality of service.

Different from the other reviewed research papers, Jing Lu and Jonathan Turner in [31] modelled a requested virtual network query by a directed graph. They used a directed graph instead of an undirected one, since their requested link bandwidth capacities are not residual, and so they are not the same in both directions.

In summary, it can be concluded that the most important attributes for substrate and virtual nodes are the CPU capacity, the memory size, the node network processor rate and the location. While the most important attributes for substrate and virtual links are the bandwidth capacity, the cost, delay, jitter, the loss rate, the length, QoS parameters and its geographic coordinates. The same attributes used for a substrate network can be used for a virtual network query. However, a substrate model describes the available amount of the attributes, while a virtual network query specifies the maximum/minimum of the attribute. A summary of the attributes and the purpose of their embedding algorithm are shown in Table 2.1. Each of the above studies used a simple model while their authors specified a model for their special work. This makes a model easy to work with. Nonetheless, there is not a standard comprehensive model which considers all of the attributes for substrate/virtual nodes and links in order to fully express the networks mathematically. By having a standard 
comprehensive model for substrate/virtual networks, all the researchers in the area of VNEs will use the same model while customizing it for their special work. This helps to have a uniform model, as a basic block, which is understandable to every researcher in this area. Consequently, it is required to design a comprehensive mathematical model for substrate/virtual networks in VNEs.

Table 2.1: Node and link attributes in VNEs

\begin{tabular}{|c|c|c|c|}
\hline Attribute & Network Element & Embedding Algorithm's Concerns & References \\
\hline CPU Capacity & $\begin{array}{l}\text { Node } \\
\text { (Server/ } \\
\text { Router) }\end{array}$ & $\begin{array}{l}\text { Path splitting, } \\
\text { Embedding over multiple substrates, } \\
\text { Decentralized embedding }\end{array}$ & [28-30] \\
\hline $\begin{array}{l}\text { Network Processor } \\
\text { Rate }\end{array}$ & $\begin{array}{c}\text { Node } \\
\text { (Router) }\end{array}$ & Embedding over multiple substrates & {$[30]$} \\
\hline Location & Node & $\begin{array}{l}\text { Path Splitting, } \\
\text { Embedding over multiple substrates, } \\
\text { Shortest path routing, } \\
\text { Traffic engineering }\end{array}$ & {$[29-31]$} \\
\hline Bandwidth & $\begin{array}{l}\text { Node, } \\
\text { Link }\end{array}$ & $\begin{array}{l}\text { Path Splitting, } \\
\text { Embedding over multiple substrates, } \\
\text { Decentralized embedding }\end{array}$ & {$[28-30]$} \\
\hline Cost & Node,Link & $\begin{array}{l}\text { Embedding over multiple substrates, } \\
\text { Decentralized embedding }\end{array}$ & {$[28,30]$} \\
\hline Delay & Node, Link & Decentralized embedding & {$[28]$} \\
\hline Jitter & Link & Decentralized embedding & {$[28]$} \\
\hline Time Stamp & Node, Link & Load Balancing & {$[32]$} \\
\hline Length & Link & $\begin{array}{l}\text { Routing, } \\
\text { Shortest path routing, } \\
\text { Traffic engineering }\end{array}$ & {$[31]$} \\
\hline $\begin{array}{l}\text { Geographic } \\
\text { Coordinates }\end{array}$ & Node, Link & $\begin{array}{l}\text { Routing, } \\
\text { Embedding over multiple substrates }\end{array}$ & {$[30]$} \\
\hline
\end{tabular}

\section{Embedding Models}

A virtual network embedding problem is to find the best match for a requested virtual network to a actual physical substrate, while satisfying all virtual network customers' necessities as well as the substrate constraints. In this regard, an embedding process 
can be modelled as a mapping from $G_{n}$ onto a subset of $G_{s}$ such that the constraints are satisfied.

$$
\text { Mapping }: G_{n} \rightarrow\left(V_{s}^{\prime}, p_{s}^{\prime}, \check{A}\left(V_{n}\right), \check{A}\left(E_{n}\right)\right)
$$

Where, $V_{s}^{\prime} \subset V_{s}$ and $p_{s}^{\prime} \subset p_{s}^{T}$, and $p_{s}^{T}$ is the set of all the possible paths in a substrate network. Besides, $\check{A}\left(V_{n}\right)$ and $\check{A}\left(E_{n}\right)$ are attributes for allocated virtual nodes and links, respectively.

A virtual network embedding problem is modelled as a single problem in Equation 2.3. However this problem has been decoupled into a node embedding problem and a link embedding problem in some papers [28, 29,32], as the followings:

1. Node embedding: In a node embedding problem we have to find a set of substrate nodes that supports a customer's requirements. $\ddot{N}\left(v_{n}^{k}\right)$ denotes a set of substrate node candidates for $v_{n}^{k} . v_{n}^{k}$ is the $k$ th virtual node in the $n$th $\mathrm{VN} . \ddot{N}\left(v_{n}^{k}\right)$ includes at least one substrate node which satisfies the requested customer's requirements for $v_{n}^{k}$ [28]. This has been described mathematically by considering only the node capacity requirements, in Equation 2.4. Therefore, $v_{s}^{i}$, that is the $i$ th substrate node, is a candidate in the case its available capacity $C\left(v_{s}^{i}\right)$ is equal or greater than the minimum requested capacity $\hat{C}\left(v_{n}^{k}\right)$ for $v_{n}^{k}$. Note that the capacity might represent different network metrics like the processing capacity, bandwidth, delay, a memory, etc. Then, we need to find the best mach among the node candidates, according to the objectives and constraints.

$$
\ddot{N}\left(v_{n}^{k}\right)=\left\{v_{s}^{i} \mid i \in V_{s}, C\left(v_{s}^{i}\right) \geq \hat{C}\left(v_{n}^{k}\right)\right\}
$$

2. Link embedding: The same strategy is used for choosing the substrate link or path candidates $\ddot{L}\left(l_{n}^{a, b}\right)$ for a virtual link $l_{n}^{a, b}$. It is needed to map the requested virtual links between allocated virtual nodes, determined by the previous problem. A virtual link can be mapped onto physical paths. However, all the links on the path must support the requested constraints of the virtual link. Note that if traffic is splittable, then a virtual link can be mapped onto multiple substrate paths. Nevertheless, if traffic is non-splittable, then the virtual link have to be mapped onto a single substrate path. Hence, in the case traffic is splittable, there can be more than one allocated traffic capacity to different virtual links of a VN in a single physical link. The problem is expressed mathematically 
in Equation 2.5. In the case the available capacity $C\left(p_{s}^{i, j}\right)$ of a substrate path $p_{n}^{i, j}$ is equal or greater than the requested capacity $\hat{C}\left(l_{n}^{a, b}\right)$, the substrate path will be a candidate for a virtual link $l_{n}^{a, b}$. In this case, it is assumed that the ath virtual node is allocated in $v_{s}^{i}$ and the $b$ th virtual node is allocated in $v_{s}^{j}$. Afterwards, we need to find the best mach among the link candidates, according to the objectives and constraints.

$$
\ddot{L}\left(l_{n}^{a, b}\right)=\left\{p_{s}^{i, j} \mid p_{s}^{i, j} \in p_{s}^{T}, C\left(p_{s}^{i, j}\right) \geq \hat{C}\left(l_{n}^{a, b}\right)\right\}
$$

Here, node embedding and link embedding problems are solved separately. In each of them, an algorithm finds the node or link candidates and another algorithm will find the best match of candidates based on the defined objectives. Decoupling the VNE embedding problem into a node mapping problem and a link mapping problem, decreases the complexity of the main problem. Nonetheless, it might not deliver the most optimum results in regard to an embedding's objective.

\section{Embedding Techniques and their Objectives}

A VNE embedding problem with multiple constraints is $\mathcal{N} \mathcal{P}$-hard [15]. Therefore, optimization programs might not be scalable to large VNEs. Hence, researchers came up with both approaches of optimization programs and heuristic algorithms to solve the problem. In this section, we review some of the state-of-the-art VNE embedding methods, and their defined objectives.

We can categorize VNE embedding solutions, regardless of their objectives, in two categories, static solutions and dynamic solutions. A static embedding method, like $[31,32,35]$, is a type of embedding technique that allocation is fixed during the lifetime of virtual networks. In this regard, SPs know every virtual network request before the allocation phase, and it will not accept any new VN demand during the network's operation time. Nevertheless, this type of embedding algorithm is not efficient, because the reserved capacity may remain idle while there are new VN demands coming from customers. On the other hand, a dynamic embedding method, like [28, 29,32-34], is an online embedding solution that can allocate the physical resources to VN demands dynamically during the time. In this category, SPs map the virtual network queries onto the physical resources, as soon as receiving new requests form VN users. So, a dynamic VNE embedding solution is more efficient than a static 
VNE embedding method. In addition, we can also categorize VNE embedding solutions in regard to an underlay substrate network. Most of the embedding algorithms designed to cover a substrate network that handled by a single InP. However, the demand may need to be mapped onto a substrate network that spans multiple InPs. To the best of our knowledge, [30] is the only study on virtual network embedding over a substrate network with multiple InPs.

Different research studies determine various objectives for their VNE embedding problems. A common VNE embedding objective is minimizing embedding cost, or maximizing the embedding revenue. However, some of the studies defined other embedding objectives like maximizing the total network utilization, or maximizing the total allocated virtual links bandwidth, etc. We study some of them in the followings:

Cost as an Embedding Objective The most common objective for a VNE embedding process in recent research studies is minimizing the embedding cost, or maximizing the embedding revenue. Several papers defined the embedding or mapping cost of a virtual network request, and proposed optimization programs and heuristic algorithms in order to map the physical resources with the minimum cost or maximum revenue. We review some of the recent embedding methods and their defined embedding cost in the followings:

Authors in $[30,32,37,38]$ presented the embedding cost of a virtual network query onto a substrate network as the summation of costs of substrate resources assigned to a virtual network query. This is shown in Equation 2.6.

$$
\grave{c}\left(G_{n}\right)=\sum_{(i, j) \in E_{s}}\left\{\grave{c}\left(l_{s}^{i, j}\right) \sum_{(a, b) \in E_{n}} \check{C}_{b}^{i, j}\left(l_{n}^{a, b}\right)\right\}+\sum_{k \in V_{n}} \grave{c}\left(v_{s}^{i}, v_{n}^{k}\right) \check{C}\left(v_{n}^{k}\right)
$$

Where $\grave{c}\left(G_{n}\right)$ is the total embedding cost of $G_{n}$. $\grave{c}\left(l_{s}^{i, j}\right)$ and $\grave{c}\left(v_{s}^{i}, v_{n}^{k}\right)$ stands for the unit cost of a substrate link $l_{s}^{i, j}$ and a substrate node $v_{s}^{i}$ hosting the $k$ th virtual node $v_{n}^{k}$, respectively. $\check{C}_{b}^{i, j}\left(l_{n}^{a, b}\right)$ denotes the amount of allocated traffic capacity in a substrate link $l_{s}^{i, j}$ to a virtual link $l_{n}^{a, b}$. Bedsides, $\check{C}\left(v_{n}^{k}\right)$ denotes the allocated capacity (processing, etc.) to $v_{n}^{k}$. The first summation in Equation 2.6 is responsible for the virtual link embedding cost, and the second summation is responsible for the virtual node embedding cost. The total link embedding cost is the multiplication of each physical link's unit cost and the total allocated traffic capacity to virtual links in the 
link. So, the physical link that no virtual link is allocated in it, costs zero. However, the total virtual nodes embedding cost is the multiplication of the unit cost of each physical node hosts a virtual node and the allocated capacity to the virtual node. The authors used different optimization techniques to minimize the embedding cost. Note that this model does not allow a virtual node to be mapped onto multiple substrate nodes. So, every virtual node can be embedded onto only one substrate node. This method does not enable the providers to split the computation virtual nodes to multiple substrate nodes. Besides, they did not define the unit cost for physical nodes and links.

$\mathrm{Yu}$ and others in [29] suggested a dynamic approach for the virtual link embedding by mapping a virtual link to multiple substrate paths with a flexible path-splitting ratio. This technique re-optimizes the paths by arriving new VN request from VN customers. The objective in [29] is to maximize the revenue. Therefore, they introduced two terms, revenue and cost for a VN request. They presented the revenue that corresponds to the economic benefit of accepting VN requests, while their proposed cost formulation considers the embedding costs in respect to the mapping with path-splitting. The revenue is defined by $\grave{r}\left(G_{n}(t)\right)$ for serving a VN request of $G_{n}$ at time $t$. Therefore, the long-term average revenue can be derived by:

$$
\lim _{x \rightarrow \infty} \frac{\sum_{t=0}^{x} \grave{r}\left(G_{n}(t)\right)}{x}
$$

The revenue can be defined in various ways according to economic models. In [29], they focused on the demanded bandwidth and CPU capacities as the main substrate network resources. Then the natural source of revenue for a VN request would be to the weighted sum of revenues for the bandwidth and CPU capacities, each of which is proportional to the amount of the requested resources. In this regard, the revenue $\grave{r}\left(G_{n}(t)\right)$ for a VN request of $G_{n}$ at any particular time $t$ that a virtual network $G_{n}$ is running, can be calculated by:

$$
\grave{r}\left(G_{n}(t)\right)=\sum_{(a, b) \in E_{n}} \hat{C}_{b}\left(l_{n}^{a, b}\right)+\psi \sum_{k \in V_{n}} \hat{C}_{c}\left(v_{n}^{k}\right)
$$

$\hat{C}_{b}\left(l_{n}^{a, b}\right)$ and $\hat{C}_{c}\left(v_{n}^{k}\right)$ are the bandwidth and the CPU capacity requirements for a virtual link $l_{n}^{a, b}$ and a virtual node $v_{n}^{k}$, respectively. $\psi$ is tuneable weight that allows the provider to strike a balance between the relative costs of the two classes of resources. We notice that the link bandwidth revenue is not affected by substrate paths that 
virtual links are mapped onto them. In another words, a physical distance or a number of hops in the mapped paths does not have any effect on the link bandwidth revenue.

They also in [29] defined the cost of a VN request with respect to the pathsplitting. The link bandwidth cost for a VN request should be defined to reflect the entire amount of the link bandwidth capacity used to map the request onto a physical substrate network. For a single virtual link in the request, it would be natural to use the allocated bandwidth capacity multiplied by the length of the substrate path that the virtual link is mapped onto. With the path splitting, we count the bandwidth capacity allocated in each path to the virtual link and sum them up. Thus, the cost of the link embedding $\grave{c}\left(E_{n}\right)$ of a virtual network $G_{n}$ is defined by:

$$
\grave{c}\left(E_{n}\right)=\sum_{(a, b) \in E_{n}} \sum_{(i, j) \in \check{p}_{n}^{a, b}} \check{C}_{b}^{i, j}\left(l_{n}^{a, b}\right)
$$

Where $\check{p}_{n}^{a, b}$ is the substrate path a virtual $\operatorname{link} l_{n}^{a, b}$ is mapped onto, and $\check{C}_{b}^{i, j}\left(l_{n}^{a, b}\right)$ is the amount of traffic capacity allocated to $l_{n}^{a, b}$ in a physical link $l_{s}^{i, j}$.

Similarly, the cost of node embedding $\grave{c}\left(V_{n}\right)$ of a $G_{n}$ can be found by:

$$
\grave{c}\left(V_{n}\right)=\sum_{k \in V_{n}} \check{C}_{c}\left(v_{n}^{k}\right)
$$

Where $\check{C}_{c}\left(v_{n}^{k}\right)$ is the amount of the sCPU capacity allocated to a virtual node $v_{n}^{k}$. Therefore in order to calculate the total embedding cost $\grave{c}\left(G_{n}\right)$ of $G_{N}$, we need to sum the link embedding cost $\grave{c}\left(E_{n}\right)$ and the node embedding cost $\grave{c}\left(V_{n}\right)$. Thus, $\grave{c}\left(G_{n}\right)=$ $\grave{c}\left(E_{n}\right)+\grave{c}\left(V_{n}\right)[29]$.

The above mentioned formulations, in Equations 2.6, 2.8, 2.9, and 2.10, calculate the cost of embedding based on the capacities allocated to a virtual network in a substrate network, and the revenue based on the requested capacities. Therefore, by increasing the allocated capacity, the embedding cost will be increased in respect to the substrate weights. This is a logical relationship between the embedding cost and the allocated capacities. It works well when a virtual network customer asks for a specific amount of bandwidth for each of the virtual nodes and links. However, in some cases virtual network customers may only concern about other constraints like a link's delay or a node's location, and they do not ask for a specific amount of CPU capacity or even link bandwidth. Therefore, in this situation, the above formulation 
is not able to calculate the embedding cost. Some modifications are needed to make a more comprehensive embedding cost formulation. This is necessary in order to make us able to calculate the embedding cost in the case of receiving any type of virtual network query from customers, with any kind of constraints. To make this happen, first, it needs to find all the possible constraints and attributes the customers might ask in regard to their virtual network query. Second, it needs to derive different possible objectives, SPs and InPs may have. Third, the relationships between the different metrics and the objectives have to be derived. Finally, it is possible to have a general comprehensive mathematic formulation which is able to calculate the embedding cost with variety of constraints and performance metrics that possible to be disabled or enabled as desired.

Luzgachev in [35] concerned about the embedding problem in design of VPNs, for unicast and multicast connections to maximize the total revenue. The proposed technique is static and considers final patterns. If a connection is set up, "it will earn revenue, but it will also cause loss in future expected revenue owing to the additional blocking that its presence causes" [35]. Therefore, they defined the following equation which calculates the long-term average revenue for a virtual private network, in terms of a unicast connection. So, if the embedding objective is maximizing the revenue, it is possible to benefit from optimization algorithms by setting the following equation as the objective function and defining the necessary constraints.

$$
\grave{r}\left(G_{n}\right)=\sum_{(i, j) \in \check{p}_{n}^{a, b}} \grave{r}_{u} \check{C}_{b}^{i, j}\left(l_{n}^{a, b}\right)\left(1-\dot{b}\left(\hat{C}_{b}\left(l_{n}^{a, b}\right)\right)\right)
$$

A VPN unicast connection will be accepted if there is an enough traffic capacity for the requested connection in all the substrate links across the path. In the case there is not enough traffic capacity in a link of the proposed path $\check{p}_{n}^{a, b}$, the request will be blocked. The blocking probability $\dot{b}\left(\hat{C}_{b}\left(l_{n}^{a, b}\right)\right)$ for the requested amount of bandwidth $\hat{C}_{b}\left(l_{n}^{a, b}\right)$ for an $l_{n}^{a, b}$ will be checked over each substrate link $l_{s}^{i, j}$ of the proposed path $\check{p}_{n}^{a, b}$. Therefore, $1-\dot{b}\left(\hat{C}_{b}\left(l_{n}^{a, b}\right)\right)$ is the acceptance probability of the requested virtual bandwidth capacity. Besides, $\grave{r}_{u}$ stands for the revenue earned per carried call per unit time, and $\check{C}_{b}^{i, j}\left(l_{n}^{a, b}\right)$ denotes the allocated bandwidth capacity to an $l_{n}^{a, b}$ in a substrate link $l_{s}^{i, j}$. Consequently, Equation 2.11 shows the long-term average revenue for a VPN can be calculated with multiplying the acceptance probability of a requested virtual connection by the allocated bandwidth capacity and the unit revenue. The good 
point of this method is considering the blocking probability in revenue calculation, while the other papers did not concern about this probability. Nonetheless, this is a static calculation in which changing the allocated bandwidth and the number of virtual networks do not affect the revenue. While, it is important to have a dynamic formulation as the topologies are changing over time. Besides, it is needed first to derive a revenue unit, which is not discussed in this paper. Moreover, this paper, as the same as previous discussed ones, did not consider other forms of network performance metrics like delay, in order to calculate the revenue.

In the same way as [29], He and others in [34] used a dynamic approach over multiple paths to maximize the aggregate performance across virtual networks. In this architecture, each substrate link periodically re-assigns bandwidth shares between its virtual networks, while at a smaller time scale, each virtual network runs an independent protocol to maximize their own performance. So, each virtual edge updates the path rates based on the local performance objective, the congestion level on its virtual paths, and possibly other constraints. While performance objectives and other constraints can differ, all virtual networks are subject to the bandwidth constraints [34]. They defined a term $\grave{c}_{c}\left(l_{n}^{a, b}, t\right)$ in order to show the congestion price for a virtual link $l_{n}^{a, b}$, at time $t$. In Transmission Control Protocol (TCP) congestion control, the link congestion prices are summed up over a path to calculate the endto-end packet loss or delay. Authors in [34] used the following equation in order to update the congestion prices of a virtual link, periodically.

$$
\grave{c}_{c}\left(l_{n}^{a, b}, t+R T T_{L}\right)=\grave{c}_{c}\left(l_{n}^{a, b}, t\right)-\psi\left(\check{C}_{b}\left(l_{n}^{a, b}\right)-r\left(l_{n}^{a, b}, t\right)\right)
$$

Therefore, the congestion price for each of the virtual links, will be updated every $R T T_{L}$ (the longest Round Trip Time (RTT) of a network), based on the difference between the virtual link traffic load $r\left(l_{n}^{a, b}, t\right)$ at time $t$, and the allocated bandwidth capacity $\check{C}_{b}\left(l_{n}^{a, b}\right)$ to the virtual link. The step-size $\psi$ moderates the magnitude of the update, and reflects the tradeoff between convergence speed (largest step-sizes) and stability (small step-sizes). Therefore, the local performance objective is to minimize the congestion price. This is a brilliant idea to define local objectives while we have a global objective for the whole network. The local objective and the the global one should be in line. This helps the embedding algorithms to achieve their objectives more effectively. However, it is required to have an initial congestion price in order to make the virtual links able to calculate the congestion price periodically. We discuss 
their global objective in the next section.

Other Embedding Objectives Some research studies defined other embedding objectives, such as maximizing the allocated bandwidth capacity, maximizing the allocated CPU capacity, etc. We review some of them in the followings:

As it is discussed, Jiayue He and others in [34] proposed a dynamic embedding algorithm for VNEs. We reviewed their local objective that minimizes the congestion price, in the previous section. Nevertheless, they also defined a global objective that is to optimize the aggregate utility of all the involved virtual networks. They defined the aggregate utility of all the involved virtual networks as in Equation 2.13.

$$
\sum_{\left\{n \mid G_{n} \in \Phi\right\}} W\left(G_{n}\right) g f\left(G_{n}\right)
$$

Where $W\left(G_{n}\right)$ shows the weight of the $n$th virtual network, $g f\left(G_{n}\right)$ is a general function which will be defined based on a traffic class for the $n$th virtual network, while its inputs are the actual data rate $r$ and the allocated bandwidth capacity $\check{C}_{b}$. $\Phi$ denotes the set of all the involved VNs. As one example, the delay-sensitive traffic may wish to choose the paths with low propagation delay and keep the queues small to reduce the queuing delay. While, the throughput-sensitive traffic may wish to maximize aggregate utility as a function of rate [34]. This approach makes sense as it has different formulations for different traffic classes. However, the previous approaches did not consider the delay-sensitive traffic. Their objective is to get the highest traffic rate for each of the defined classes. In their method there is a defined priority for each of virtual networks. This helps to have multi-level priority based VNE. The general problem objective (Equation 2.13) as well the local performance objective (Equation 2.12) brought a good objective combination in in order to get to the optimum point.

Assuming traffic is throughput-sensitive, the following function defines $g f\left(G_{n}\right)$ :

$$
g f\left(G_{n}\right)=\sum_{a \in V_{n}} \log \left(\sum_{b \in V_{n}} r\left(\check{p}_{n}^{a, b}\right)\right)-\psi \sum_{(a, b) \in E_{n}} \exp \left(U\left(l_{n}^{a, b}\right)\right)
$$

where

$$
U\left(l_{n}^{a, b}\right)=\frac{\alpha\left(l_{n}^{a, b}\right) r\left(l_{n}^{a, b}\right)}{\check{C}_{b}\left(l_{n}^{a, b}\right)}
$$


$r\left(\check{p}_{n}^{a, b}\right)$ denotes the traffic rate of the allocated path for an $l_{n}^{a, b} \cdot r\left(l_{n}^{a, b}\right)$ shows the traffic rate for a virtual link $l_{n}^{a, b} . \alpha\left(l_{n}^{a, b}\right)$ denotes whether a link is used by the virtual network or not. If a link is used $\alpha\left(l_{n}^{a, b}\right)$ is 1 , otherwise $\alpha\left(l_{n}^{a, b}\right)$ is 0 . Besides, $\check{C}_{b}\left(l_{n}^{a, b}\right)$ shows the allocated traffic capacity to an $l_{n}^{a, b}$. $\psi$ is the strike balance between maximizing utility and minimizing congestion. In [34] they set $\psi$ as 0.5. In this function (2.14) each source virtual node is maximizing its utility as a logarithmic function of its sending rate. However, to avoid congestion, each link penalizes high link utilization with an exponential function. Therefore, the objective is to increase a link's rate logarithmically while decreasing the high rates exponentially to avoid congestion. This strategy makes sense, however by getting ideas from TCP congestion control strategies, it is also possible to maximize a link's utility exponentially while using linear functions to control the congestion. Equation 2.14 can be used as an objective function of embedding algorithms in the case of throughput-sensitive traffic.

Nonetheless, assuming traffic is delay-sensitive, the following function defines $g f\left(G_{n}\right)$ :

$$
g f\left(G_{n}\right)=\sum_{a \in V_{n}} \sum_{b \in V_{n}} r\left(\check{p}_{n}^{a, b}\right) \sum_{(a, b) \in E_{n}} \alpha\left(l_{n}^{a, b}\right)\left(d_{p}\left(l_{n}^{a, b}\right)+d_{q}\left(U\left(l_{n}^{a, b}\right)\right)\right.
$$

Where $d_{p}\left(l_{n}^{a, b}\right)$ is the propagation delay of a virtual link $l_{n}^{a, b}$, and $d_{q}\left(U\left(l_{n}^{a, b}\right)\right.$ approximates the queuing delay, as a function of a link's utilization $U\left(l_{n}^{a, b}\right)=\frac{\alpha\left(l_{n}^{a, b}\right) r\left(l_{n}^{a, b}\right)}{\check{C}_{b}\left(l_{n}^{a, b}\right)}$. In their simulations they use $d_{q}\left(U\left(l_{n}^{a, b}\right)\right)=10^{-3} \exp \left(U\left(l_{n}^{a, b}\right)\right)$. Note that the delaysensitive traffic is inelastic, so it has a fixed traffic rate that needs to be met. The delay on a path is the sum of the link propagation delays and queuing delays for all links on the end-to-end path. Therefore, the end-to-end delay on a path to a sink virtual node from a source virtual node is $\sum_{(a, b) \in E_{n}} \alpha\left(l_{n}^{a, b}\right)\left(d_{p}\left(l_{n}^{a, b}\right)+d_{q}\left(U\left(l_{n}^{a, b}\right)\right)\right.$. The objective of the optimization which captures the average delay for all traffic is obtained by minimizing the products of the path rates and the associated delay [39]. In order to reflect the true behaviour of the system and derive the real delay we need to consider all the delay types the packet may take. Nonetheless, the method does not consider the processing delays and the effect of collision or congestion in the network.

Authors in [29] suggested a dynamic approach for the virtual link embedding by embedding a virtual link to multiple substrate paths with a flexible path-splitting ratio. This technique re-optimizes the paths by arriving a new VN request from VN customers. In order to keep the track of a node/link's available resources, they defined 
a mathematical function for the substrate nodes. They did not use the CPU capacity alone as a factor representing a substrate node's capacity. Because, we not only want to make sure that there is enough available CPU capacity, but also consider the bandwidth capacity to prepare for the subsequent link embedding stage [29]. Therefore, the amount of available resources for a substrate node $v_{s}^{i}$ is defined by:

$$
\breve{C}_{c}\left(v_{s}^{i}\right) \sum_{(i, j) \in \breve{L}\left(v_{s}^{i}\right)} \breve{C}_{b}\left(l_{s}^{i, j}\right)
$$

Where $\breve{L}\left(v_{s}^{i}\right)$ is the set of all adjacent substrate links of a $v_{s}^{i}, \breve{C}_{c}\left(v_{s}^{i}\right)$ is the remaining CPU resource of a $v_{s}^{i}$, and $\breve{C}_{b}\left(l_{s}^{i, j}\right)$ is the unoccupied bandwidth resource of a substrate link $l_{s}^{i, j}$. This is reasonable to consider both node CPU capacity and its adjacent bandwidth capacity, when we want to express the available resources of a substrate node. However, the other important parameters like delay are ignored.

One of the initial works on VNE embeddings is [31]. They developed a static algorithm to allocate the sufficient bandwidth capacity to virtual links in order to ensure a virtual network can handle any traffic pattern in a general set of traffic constraints. Nevertheless, a static embedding algorithm is not efficient in a dynamic network which the virtual topology is changing all the time. A dynamic algorithm is required to adapt itself during the time.

Houidi and others in [28] proposed a dynamic distributed VNE embedding architecture, which does not have the problems of centralized embedding systems, such as scalability limitations, high latency and single point of failure. Their design is based on autonomous agents to exchange messages between each other in order to implement a distributed VNE embedding algorithm. Therefore, they also proposed a protocol to handle the messaging between node agents. The main benefit of this approach is its architecture which is decentralized.

Yong Zhu and Mostafa Ammar in [32] studied two VNE embedding problems. First, they suggested a heuristic algorithm for VN assignment when the allocation is fixed during the VN's life time. As in a static approach, we know all the virtual network demands at first, they developed a static algorithm to get the low and balanced load on both substrate nodes and links. Second, they discussed a dynamic algorithm for a VN assignment with reconfiguration. As in a dynamic approach a virtual network may leave or a new virtual network demand may come, the designed algorithm is a dynamic and adaptive. This algorithm prioritizes the reconfiguration 
for the most critical VNs. One of their embedding objectives is to have a balanced stress across a substrate network to avoid hot spots and reduce congestions. Therefore, their virtual network allocating algorithm tries to maintain low and balanced stress among all substrate links and substrate nodes. In this regard, they defined a node stress ratio at time $t \tilde{s}\left(V_{s}, t\right)$ as the ratio of the maximum node stress and the average node stress across the whole substrate network. Similarly, a link stress ratio at time $t \tilde{s}\left(E_{s}, t\right)$ is the ratio of the maximum link stress and the average link stress.

$$
\begin{gathered}
\tilde{s}\left(V_{s}, t\right)=\frac{\max _{i \in V_{s}} \check{N}\left(v_{s}^{i}, t\right)}{\left(\sum_{i \in V_{s}} \check{N}\left(v_{s}^{i}, t\right)\right) /\left|V_{s}\right|} \\
\tilde{s}\left(E_{s}, t\right)=\frac{\max _{(i, j) \in E_{s}} \check{L}\left(l_{s}^{i, j}, t\right)}{\left(\sum_{(i, j) \in E_{s}} \check{L}\left(l_{s}^{i, j}, t\right)\right) /\left|E_{s}\right|}
\end{gathered}
$$

Where $\check{N}\left(v_{s}^{i}, t\right)$ is the number of virtual nodes that are assigned in a substrate node $v_{s}^{i}$ at time $t$, and $\check{L}\left(l_{s}^{i, j}, t\right)$ is the number of virtual links allocated in a substrate link $l_{s}^{i, j}$ at time $t$. The good point about this formulation is that it is a dynamic expression of stress, which is changing over the time. From the definition, we can see $\tilde{s}\left(V_{s}, t\right) \geq 1$ and $\tilde{s}\left(E_{s}, t\right) \geq 1$ for all $t>0$. The stress ratio is a proper metric to evaluate the load balancing performance since a smaller stress ratio indicates that the stress is more balanced. If the node (or the link) stress ratio is 1 , the network achieves optimal load balancing since all substrate nodes (or links) have the same stress. Although the link stress ratio reflects the load balancing performance, it may not be sufficient to reveal the true efficiency of a VN assignment. It indicates that assignments with different efficiency can have the same $\tilde{s}$. The key factor here is the maximum link stress. Therefore, instead of using the stress ratio, they use the maximum link/node stress as the performance metrics, i.e., they wanted to minimize both the maximum node stress and maximum link stress. Due to the heterogeneity of the substrate topology, it may not be possible to achieve optimal stress for both substrate nodes and substrate links at the same time.

[33] is also one of the initial adaptive and dynamic embedding algorithms. This approach is a fault-tolerant embedding algorithm which relies on substrate node agents to cope with the failures. In the case of a substrate node failure, a message will be broadcasted to all the candidate substrate nodes. Afterwards, each substrate node that receives the failure message needs to check the similarity of the failed node 
attributes with itself. A substrate node which has the most similar attributes to the failed node will be chosen as the replaced node. This is a type of embedding process. They used a mathematical function $\Delta$ in order to consider and combine all possible non-functional node attributes types (binary, nominal or interval). $\Delta$ is computed as follows:

$$
\Delta^{i, j}=\frac{\sum_{m}^{n} \kappa_{m}^{i, j} \Delta_{m}^{i, j}}{\sum_{m}^{n} \kappa_{m}^{i, j}}
$$

$\Delta^{i, j}$ calculates the dissimilarity of attributes of the $i$ th substrate node and the $j$ th substrate node, while $n$ is the number of non-functional (NF) attributes of a request node $v_{s}^{i} . \quad \Delta_{m}^{i, j}$ checks the dissimilarity of an attribute $m A\left(v_{s}^{i}, m\right)$ of a $v_{s}^{i}$ with the respective attribute of a $v_{s}^{j}$, and can be found using the attribute type. If the attribute is binary or nominal it can be calculated as follow:

$$
\Delta_{m}^{i, j}= \begin{cases}0 & \text { if } A\left(v_{s}^{i}, m\right)=A\left(v_{s}^{j}, m\right) \\ 1 & \text { if } A\left(v_{s}^{i}, m\right) \neq A\left(v_{s}^{j}, m\right)\end{cases}
$$

However, if the attribute type is interval, normalized distance such as Euclidean distance and Manhattan distance can be used [33]. $\kappa_{m}^{i, j}$ is used to express the priority coefficients of the NF attributes for nodes $v_{s}^{i}$ and $v_{s}^{j}$. Consequently, a multi-level priority is assigned to the NF attributes (in this case the $m$ th one) of each virtual node. By using this function it is possible to find the most similar substrate node to the failed one. This is an effective method in order to find the most similar replaced node for a failed one. So, if all the substrate nodes of a network calculate their similarity rate to a failed node, it is possible to find the best match. Nevertheless, this is a time consuming approach. It takes time in order to notice the failure, broadcasting a message to all the active substrate nodes, calculating the similarity of each node and then finding the best match among all the available ones. However, one of the most important parameters in terms of fault-tolerant networks is to recover the service, fast. Moreover, their approach is not scalable to large network sizes. In the case we have a large virtual network over a large substrate network, it is not effective to broadcast a message to all the substrate nodes and find the most similar one. Furthermore, virtual networks might get mapped to multiple substrate networks. Therefore, in the case of node failure in one of the substrate network, based on the above approach, a message should be broadcasted to all the involved substrate 
networks' nodes and ask for the similarity. Nevertheless, majority of the substrate networks do not reveal their specifications to the others due to the policies they have. Hence, as the fault recovery is essential in any type of network and even it is more important in VNEs, because there are more than one network over a single physical network, it is necessary to have a fast reliable effective failure recovery approach. The approach should be dynamic as the virtual network topologies are changing over the time, and then the active network elements as well as their attributes are changing by time. The approach also should support an InP and customers' constraint in failure time.

\subsubsection{Network Performance in VNEs}

In network virtualization environments, an SP may map a virtual network request onto multiple InPs and not only one InP. Therefore, a virtual network may span multiple substrate networks, while each has dissimilar network performance. Consequently, it is needed to analyze the end-to-end network performance and study its dependency to the each of network's performance. This is discussed in this section.

Network performance is the measure of the quality of network service. There are different metrics that reflect the network performance. The two main network performance metrics are throughput and delay. Throughput is a measure of how much traffic is successfully received at an intended destination [40]. Delay is the time that a packet takes to travel from the transmitter to the receiver. We study the network performance for VNEs and the overheads a VNE may add, in this section.

\section{End-to-End Service Delivery}

As explained in Section 2.2.1, the role of ISPs is decoupled into two different entities in VNEs. The first one that handles physical infrastructure is called an InP, and the second one that provides the end-to-end service to virtual network customers is called an SP. An end-to-end virtual service in VNEs may span multiple InPs. In consequence, an end-to-end service delivery in VNEs consists of series of service components which are logical abstraction of substrate networks provided by several InPs to an SP.

There are lots of papers in the literature that discuss network virtualization, however understanding the end-to-end service delivery performance in virtualized network environments is still an open topic which needs analytical modelling [41]. There are 
few papers that reviewed analytical modelling of the end-to-end service delivery performance in virtualized network environments.

Qiang Duan in [41] and [42] brought an analytical model for the end-to-end traffic delivery in VNEs by benefiting from network calculus theory [43]. In order to model the whole end-to-end service, it is possible to break it up into multiple components of service for each of the InPs, and define the capability function for every service. Therefore, combining all of the service components makes the whole end-to-end service delivery in a VNE. The capability function denotes the lower band of networking capacity an SP expects from an InP. Hence, by using the concept of service curve from network calculus theory [43], the author in [41] derived a traffic capability function for each of the defined service components. Let $R(t)$ be the arrival traffic load by time $t$ in a networking session and $E(t)$ the traffic departs from a service component by time $t$. Given a non-negative, non-decreasing function of time $S(t)$, where $S(0)=0$, it is possible to say the service element guarantees a traffic capability function $S(t)$ for the session, if for any $t \geq 0$ in the busy period of component:

$$
E(t) \geq R(t) * S(t)=\int_{-\infty}^{+\infty} R(\tau) S(t-\tau) \mathrm{d} \tau
$$

According to the definition in [43], considering a system with the input and output function of $R$ and $E$, we say that the system offers a service curve of $S$ if and only if the output $E$ is equal or greater than the convolution of input $R$ and service curve $S$. This has been shown in the case of our scenario in Equation 2.22. A traffic capability function gives the minimum amount of traffic capacity offered to a networking session by a service component, which is an abstraction of a network infrastructure. Such a function is a general function of time that specifies the service traffic capacity through the relation between arrival and departure traffic at a service component; therefore it is independent of network architectures and technologies, and applicable to various heterogeneous network infrastructures in the Internet [41].

A single service component model has been studied. However, to analyze an endto-end service delivery in network virtualization environment, we need to consider all the service components in an end-to-end connection. From the network calculus theory, a service curve guaranteed by a series of tandem services can be obtained from the convolution of the series curves guaranteed by these services. Therefore, the traffic capability function $S_{e}(t)$ for an end-to-end virtual connection, which includes multiple services over different InPs, is the convolution of all of traffic capability 
functions $S_{i}(t)$, as calculated in Equation 2.22. $S_{i}(t)$ is the $i$ th traffic capability function.

$$
S_{e}(t)=S_{1}(t) * S_{2}(t) * \cdots * S_{n}(t)
$$

Consequently, an end-to-end service in network virtualization environments is dependent to each of the service components offered by any underlying substrate network along the end-to-end service. Although the above discussion is valid, it has a very general view to the service each of the substrate networks offer. Each of the substrate networks does not have the same rate on all of their links, so setting a single rate or latency for a service component that represents the underlying substrate network, does not reflect its real behaviour. Besides, normally the virtual network customers have different bandwidth requirements over each of the virtual links. In this case, the above analytical model over-provision the allocated bandwidth amount since it asks for the maximum bandwidth requirement of the customers. Finally, a general model that considers other end-to-end performance metrics, like delay, is necessary.

\section{Throughput}

Throughput is one of the important network performance metrics. Throughput is a measure of how much traffic is successfully received at an intended destination [40]. Ideally, the throughput is equal to the instantaneous load over a channel in the case the channel is error free. But, in fact the throughput is less than actual traffic load over a channel when the system is working below the highest capacity [40]. However, in majority of the papers throughput is considered as the actual instantaneous traffic over a communication channel. We use the same definition. In regards of throughput, some other related metrics also come up. The capacity, which is maximum throughput, and also utilization, that is a ratio of throughput and a capacity.

Throughput is an important performance metric in VNEs. As discussed in Section 2.2.1, various virtual network embedding algorithms map the best substrate candidates to a virtual network query. The maximum throughput for each of the virtual links in a network virtualization environment is the bandwidth allocated to that specific virtual link. In this regard, we study an end-to-end service's throughput in a VNE. Second, we review a traffic predictor for virtual links. Finally, we discuss 
overheads in a VNE, and their impacts on throughput.

As it is discussed in 2.2.2, Duan in [41] proposed a general analytical model for an end-to-end traffic delivery in network virtualization environments. The traffic capability function defined in this section gives the minimum bandwidth provided by a service to its end users. Therefore, given $S_{e}(t)$ for an end-to-end service, the minimum bandwidth $C_{b}\left(S_{e}\right)$ for the end-to-end network service can be calculated by:

$$
C_{b}\left(S_{e}\right)=\lim _{t \rightarrow \infty}\left(\frac{S_{e}(t)}{t}\right)
$$

Equation 2.24 derives the minimum bandwidth offered by a service, which is assumed in [41] that is mapped onto multiple substrate networks. In order to calculate $C_{b}\left(S_{e}\right)$, first it is needed to have the traffic rate for each of the underlying substrate networks. So, the end-to-end minimum bandwidth can be found by calculating the convolution of each of the service component functions. This model clearly shows the dependency of the end-to-end throughput in a VNE to the each of the substrate networks across a path. This is what researchers in this area need to consider. However, the model has some issues that need further study. In this model the required bandwidth for each service component is considered as the maximum bandwidth over that specific substrate network. However, in VNEs, we have different bandwidth demands for each of the virtual links and even for each physical path. Therefore, some modifications are needed in this section.

Yongtao Wei and others in $[44,45]$ modelled the traffic in a virtual link. By calculating the mean traffic rate for every time interval $\left[t_{1}, t_{2}\right),\left[t_{2}, t_{3}\right), \ldots$, we can get a time series of traffic rates $r(1), r(2), \ldots, r(t)$. So, it is possible to apply a linear predictor on that time series [45]. The same authors in [44] proposed the following predictor for $n$ items from the past history:

$$
r(t+n)=\bar{r}+\sum_{i=1}^{n} \psi(r(t-i+1)-\bar{r})
$$

Where $\bar{r}$ is the mean of the time series $r(t)$, and $\psi$ is a tuneable weight. According to the correlations the traffic rates have in different times, it is possible to predict the traffic rate for a limited future time. This predictor can be used also for the nonvirtualized networks. Therefore, for every allocated virtual link in a physical link, we can benefit from such a traffic predictor. This work triggers the necessity and also possibility of having a traffic predictor based on the correlations the traffic rates have 
over time. However, this is a simple linear predictor, which is not designed especially for VNEs. The requirements for VNEs are different to the normal networks. A VN may pass over multiple substrate networks with different properties. Besides, it is a dynamic environment that virtual networks are arriving and leaving during the time. Hence, a simple linear predictor is not effective in a VNE. However, similar predictors, but much more accurate, which are specially designed for VNEs, are essential in order to predict the upcoming traffic. This may also help VNE energy-saving solutions to save power more effectively. In order to formulate a predictor, first it is needed to understand the dynamic traffic behaviour in a VNE. Afterwards, we have to study its stochastic behaviour.

One of the important issues in VNEs is the overhead traffic virtual networks adds to the packets. This affects the throughput, directly. Tsugawa and Fortes in [46] reviewed the performance of Overlay Networks (ON) which is a type of virtual networks. The overlay networks add some information to the IP packet which is considered as ON overhead $O_{o}$. In the case IP packets tunnelled using TCP/IP the travelling message will have two TCP/IP headers in addition to the ON header. The authors in their paper [46] considered the size of ON header as 52 bytes. Therefore, the throughput expression will be different due to added overhead. The maximum throughput $r\left(l_{n}^{a, b}\right)$, which can be experienced by an application uses a virtual link $l_{n}^{a, b}$, is a fraction of the allocated capacity $\check{C}_{b}\left(l_{n}^{a, b}\right)$ to the virtual link. This is formulated in Equation 2.26.

$$
r\left(l_{n}^{a, b}\right)=\frac{M T U-H_{i}-H_{t}-O_{o}}{M T U+O_{e}} \times \check{C}_{b}\left(l_{n}^{a, b}\right)
$$

Where transport header $H_{t}$ has 8 bytes in the case of User Datagram Protocol (UDP) and 32 bytes in the case of TCP (in modern Linux kernels, due to the need for optional header fields to support window scaling, selective acknowledgements and timestamps), and IP header $H_{i}$ is 20-bytes long. Ethernet overhead $O_{e}$ is 38 bytes: 14 bytes for the header, 4 bytes for frame checksum, 12 bytes for inter-frame gap and 8 preamble bytes. The numerator represents the length of data sent by applications i.e., the IP Maximum Transmission Unit (MTU) without headers. The denominator is the data length effectively transmitted in the physical layer, which includes Ethernet overhead. The maximum throughput decreases linearly at a rate of $0.65 \mathrm{Mbps}$ per VN overhead byte [46].

In some cases the IP packet may get fragmented due to MTU size. This results 
of transmitting two fragmented IP packets instead of one. Then the throughput can be expressed with following function:

$$
r\left(l_{n}^{a, b}\right)=\frac{M T U-H_{i}-H_{t}}{M T U+O_{e}+\max \left\{84, O_{e}+H_{i}+O_{o}\right\}} \times \check{C}_{b}\left(l_{n}^{a, b}\right)
$$

where the expression $\max \left\{84, O_{e}, H_{i}, O_{o}\right\}$ represents the overhead of the additional frame due to the fragmentation. It is at least the minimum Ethernet frame size of 84 bytes: 64 bytes for the frame including header and checksum and 20 bytes for inter-frame gap and preamble.

Furthermore, Gad and others in [47] experimentally studied the network performance of virtualized network technologies and compared the performance of different technologies. However, they have also discussed throughput theoretically in order to check their experiments results with the theory. They stated the achievable throughput $r$ can be calculated with Equation 2.28.

$$
r=\frac{s_{l}}{s_{p}}
$$

They ignored the effect of collisions and congestions to make the calculations easier. The packet size $s_{p}$ is the sum of the overheads $O_{T}$ caused by different protocol headers, and $s_{l}$ is the actual payload size .

$$
s_{p}=O_{T}+s_{l}
$$

where the payload size $s_{l}$ is:

$$
s_{l}=M T U-\left(O_{i}+O_{t}\right)
$$

$O_{i}$, and $O_{t}$ denotes the network and transport layer overheads, respectively.

Both of the above papers $[46,47]$ have considered the overhead caused by the header added by an overlay network. Nevertheless, the above papers ignore the effect of collision and congestion for throughput calculations. Besides, there are other overheads a VNE adds due to the required signalling for handling concurrent networks. As far as we know there are few related works available in the literature. In order to find the total overhead added by a VNE, we need to consider different protocols used in VNEs and their signalling packets. So, a precise study is necessary to discuss all types of overhead in VNEs and their effects. 


\section{Delay}

One of the important network performance metrics, which is essential for virtual network customers, is delay. There are four types of delay in communication networks, transmission, propagation, processing and queuing delay. Transmission, propagation and processing delays can be calculated mathematically by having accurate information of a network. Nonetheless, this is not possible for queuing delay, as the packet arrival of the system is not a constant value. But, it is possible to calculate some probabilities with stochastic analysis to have a good approximation of delay which may be caused by packet queues in a network. This issue is essential in network virtualization as a virtual connection between two virtual nodes may pass through different physical paths. Therefore, it is needed to have analytical queuing models for virtual networks in order to predict the delays. In this regard, first, we study the end-to-end delay in VNEs according to our discussion in 2.2.2. Second, we study a queuing model that evaluates the end-to-end delay in an MPLS virtual private networks.

Considering again the discussion in Section 2.2.2, the maximum end-to-end delay performance is related directly to both the networking capacity of the system and also traffic properties of that networking session. We have discussed that the authors in [41] modelled the network bandwidth capacity by a traffic capability function $S(t)$. However, they also defined a non-negative, non-decreasing function $L(t)$, which shows the traffic load function for the networking session if:

$$
R(t)-R(i) \leq L(t-i), \quad \forall i: 0<i<t
$$

Therefore, it can be shown considering a network with traffic capability function of $S(t)$ and traffic load function of $L(t)$ the maximum delay $d$ for a network service delivery can be expressed by:

$$
d=\max (\min \{\delta: \delta \geq 0, L(t) \leq S(t+\delta)\})
$$

This reveals that the end-to-end delay in a VNE is dependent to each of service components offered by underlying substrate networks. However, this model has the same disadvantages as discussed in the previous sub-section.

Zhu and others in [48] proposed a queuing model to evaluate the end-to-end delay in MPLS virtual private networks. They believe the traffic in this type of networks 
is self-similar. Nevertheless, it is very tough to compute a queuing model with a self-similar arrival process. But, they benefited from the data available in Management Information Base (MIB) of routers to estimate the QoS metrics. The periodic statistics of packet loss rate $\left(l\left(\Upsilon^{i, j}\right)\right)$, the mean queue length $\left(E\left[q_{l}\left(\Upsilon^{i, j}\right)\right]\right)$, and utilization of a port $\left(U\left(\Upsilon^{i, j}\right)\right)$, are available in MIB of most of routers and switches [48]. Where $\Upsilon^{i, j}$ denotes the $j$ th port of the $i$ th router. Therefore, it is straightforward to calculate the end-to-end packet loss rate $l$ based on the information we have in MIB, by Equation 2.33. $\Upsilon$ denotes the set of router ports corresponding to the considered Label-Switched Path (LSP).

$$
l=1-\Pi_{\left\{(i, j) \mid \Upsilon^{i, j} \in \Upsilon\right\}}\left(1-l\left(\Upsilon^{i, j}\right)\right)
$$

Nonetheless, we know from queuing theory that the packet loss rate at $\Upsilon^{i, j}$ is the probability of having $n\left(\Upsilon^{i, j}\right)$ packets in its buffer, as expressed mathematically in Equation 2.34.

$$
l\left(\Upsilon^{i, j}\right)=P\left(q_{l}\left(\Upsilon^{i, j}\right)=n\left(\Upsilon^{i, j}\right)\right)
$$

So, the mean queue length is given by:

$$
E\left[q_{l}\left(\Upsilon^{i, j}\right)\right]=\sum_{k=1}^{n\left(\Upsilon^{i, j}\right)} k P\left(q_{l}\left(\Upsilon^{i, j}\right)=k\right)=n\left(\Upsilon^{i, j}\right) l\left(\Upsilon^{i, j}\right)+\sum_{k=1}^{n\left(\Upsilon^{i, j}\right)-1} k P\left(q_{l}\left(\Upsilon^{i, j}\right)=k\right)
$$

Hence, in a condition when the new packet can enter the buffer of $\Upsilon^{i, j}$, the observed buffer length can be calculated by:

$$
E^{*}\left[q_{l}\left(\Upsilon^{i, j}\right)\right]=E\left[q_{l}\left(\Upsilon^{i, j}\right)\right]-n\left(\Upsilon^{i, j}\right) l\left(\Upsilon^{i, j}\right)
$$

Besides, the utilization of the port can be calculated using following equation:

$$
\begin{gathered}
U\left(\Upsilon^{i, j}\right)=\frac{\lambda\left(\Upsilon^{i, j}\right) C_{b}\left(\Upsilon^{i, j}\right)}{E\left[s_{p}\right]} \\
\lambda\left(\Upsilon^{i, j}\right)=\frac{U\left(\Upsilon^{i, j}\right) E\left[s_{p}\right]}{C_{b}\left(\Upsilon^{i, j}\right)}
\end{gathered}
$$

Consequently, by considering Little's law in queuing theory and having the arrival 
mean rate of $\lambda\left(\Upsilon^{i, j}\right)$ as well as $E^{*}\left(q_{l}\left(\Upsilon^{i, j}\right)\right)$, the mean sojourn time $d\left(\Upsilon^{i, j}\right)$ which consists of queuing delay, processing delay and transmission delay, can be expressed by $\frac{E^{*}\left[q_{l}\left(\Upsilon^{i, j}\right)\right]}{\lambda\left(\Upsilon^{i, j}\right)}$, i.e.,

$$
d\left(\Upsilon^{i, j}\right)=\frac{C_{b}\left(\Upsilon^{i, j}\right)\left(E\left[q_{l}\left(\Upsilon^{i, j}\right)\right]-n\left(\Upsilon^{i, j}\right) l\left(\Upsilon^{i, j}\right)\right)}{U\left(\Upsilon^{i, j}\right) E\left[s_{p}\right]}
$$

Where $C_{b}\left(\Upsilon^{i, j}\right)$ denotes the transmission rate of port $\Upsilon^{i, j}$, and $s_{p}$ is the packet length. In the above expressions all the parameters are possible to be derived from MIB of the routers or switches, online. Hence, we are able to derive the end-to-end delay with following equation, where $d_{p}$ is the propagation delay of the LSP:

$$
d=\sum_{\left\{(i, j) \mid \Upsilon^{i, j} \in \Upsilon\right\}} \frac{C_{b}\left(\Upsilon^{i, j}\right)\left(E\left[q_{l}\left(\Upsilon^{i, j}\right)\right]-n\left(\Upsilon^{i, j}\right) l\left(\Upsilon^{i, j}\right)\right)}{U\left(\Upsilon^{i, j}\right) E\left[s_{p}\right]}+d_{p}
$$

The described method is easy to calculate the end-to-end delay because it uses the available data stored in the routers and does not need any additional measurements. Nonetheless, it considers traffic, stochastically, as a self-similar traffic and then derived the queuing model based on that. This limits the model, because the traffic in VNE may not always be self-similar. So, the model is not a general one. Besides, the mentioned strategy needs to check the data in MIB of each router or switch over different paths. This is a time-consuming approach and is not efficient, while it may not be useful in large scale scenarios as well. Thereby, first, it is needed to clarify the traffic distribution in VNE. Afterwards a model which can analytically study the system has to be derived. Thus, a queuing model, for any type of virtual networks, not only a specific kind (MPLS), which is considering different types of traffic, is required to be developed.

The reviewed and discussed studies [34,41,48] in this section, consider the transmission, queuing and also propagation delays. Nonetheless, they neglect the effect of processing delays in a VNE as well as a congestion effect. It is a real need to find out all the delays the packet takes in a VNE and formulate them. This issue is more important for VNEs, because we have more than one network over a single physical network, while a VNE adds its own overhead and signalling packets. In addition, the objective of a VNE embedding process may be defined in terms of an end-to-end delay. Hence, it is essential to derive the precise an end-to-end delay of VNEs. Therefore, we need to first find the processing delays that may happen in VNEs. Moreover, 
the dynamic congestion effect should be considered as well.

\subsubsection{Performance Modelling of Virtualized Servers}

By increasing demands of network storages and the cloud computing, data center providers need to add an extra number of servers in order to cope with the growing demand. Nonetheless, the virtualization technology slows down the infrastructure expansion by virtualizing the physical servers to handle concurrent operating systems, as well as the network. The server virtualization is a technique of running multiple virtual servers (Virtual Machines (VMs)) over a single physical server, simultaneously. Several papers in the literature discuss software implementation and operating systems' specification in the sever virtualization [49-55]. In this section, we study analytical modelling of virtualized servers' performance, according to [1]. We compare a model for a virtualized server to a model for a non-virtualized one. The analytical modelling enables us to calculate various performance metrics, such as the mean queue length, the response time, and the utilization.

\section{A Model for Non-Virtualized Severs}

The well-known queue theory can express the behaviour of a non-virtualized server. First, assume two servers are operating on two different physical machines. Each of the servers receives jobs with Poisson arrival process. $\lambda$ is the mean rate for arrival Poisson process. $\mu$ is the mean rate for exponentially distributed service times. The behaviour of each server can be expressed by $\mathrm{M} / \mathrm{M} / 1$ queuing model, independently. The state space for $\mathrm{M} / \mathrm{M} / 1$ queue is shown in Figure 2.3.

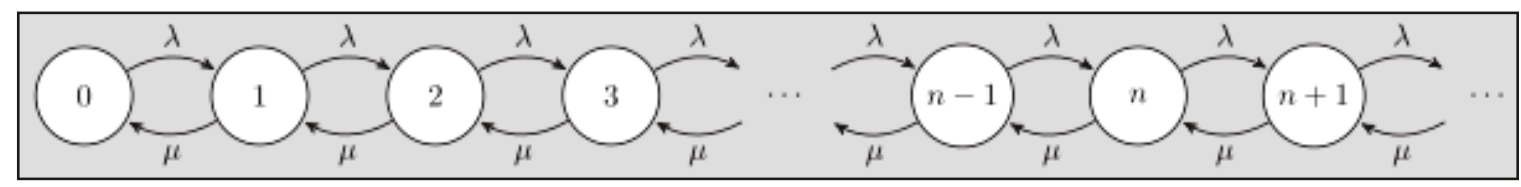

Figure 2.3: M/M/1 queue model for non-virtualized severs

Consequently, the well-known solutions for $\mathrm{M} / \mathrm{M} / 1$ calculate the performance metrics of non-virtualized servers. Considering the steady state of the queue and balance equations, Equation 2.41 calculates the probability $P(j)$ of being in state $j$ in the queue. In this scenario, state $j$ means there are $j$ jobs in each of the servers. 


$$
P(j)=\rho^{j} P_{0}=\rho^{j}(1-\rho)
$$

Where $\rho$ is the traffic intensity and it is defined by $\frac{\lambda}{\mu}$. Therefore, the mean queue length $E\left[q_{l}\right]$ or in our specific scenario the mean number of jobs in the system, throughput $r$, and the average server response-time $E[t]$ (time in the system) are possible to be calculated based on the described Markov model as follow:

$$
\begin{gathered}
E\left[q_{l}\right]=\sum_{j=0}^{\infty} j P(j)=\frac{\rho}{1-\rho} \\
r=\mu \sum_{j=1}^{\infty} P(j)=\lambda \\
E[t]=\frac{E\left[q_{l}\right]}{r}=\frac{1}{\mu-\lambda}
\end{gathered}
$$

In the case of a larger number of servers, $M / M / 1$ does not reflect the system behaviour. Therefore, we may need more complex queuing systems like $\mathrm{M} / \mathrm{M} / \mathrm{k}$, in which we have $k$ servers.

\section{A Model for Virtualized Servers}

On the other hand, in the case of a virtualized server, the calculated metrics are not the same. Therefore, it is required to model a virtualized server. Accordingly, we compare the performance of a non-virtualized server to a virtualized one.

In the second model which is also discussed in [1], we have two servers. However, different from the previous scenario, these are two virtual servers running on a single physical machine. It is also possible to model this server modification with $\mathrm{M} / \mathrm{M} / 1$ queuing model. The arrival rate is Poisson with the mean of $\lambda$ and inter-arrival times are distributed exponentially. As it is shown in Figure 2.4, there is one actual physical CPU that serves both of the virtual servers.

Consequently, the mean service rate is $\frac{\mu}{2}$. In this case, the approximate performance can be calculated in the same way as non-virtualized servers, when the service mean rate is $\frac{\mu}{2}$, and accordingly $\rho=\frac{2 \lambda}{\mu}$. Nevertheless, this method ignores the fact that when one of the virtual servers is idle, the other virtual server can benefit from the full CPU capacity. In this case the service rate will be $\mu$, not $\frac{\mu}{2}$. This issue can be 


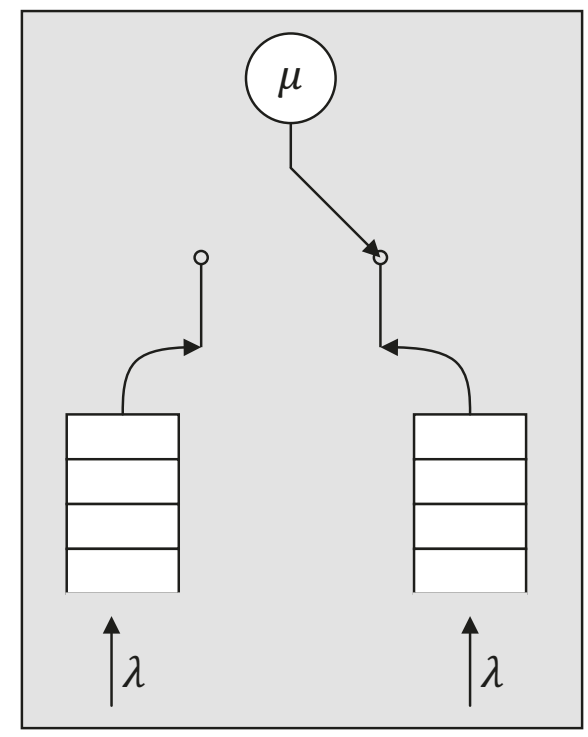

Figure 2.4: Queue model for virtual servers [1]

relatively important when the idle time for each of the virtual servers is large. Hence, the previously described model is not a true behaviour reflection from what actually happens in virtual servers. In order to solve this problem, authors in [1] developed a third model in which addresses the mentioned problem. The new model is still a Markov-chain model, however the service time switches between $\mu$ and $\frac{\mu}{2}$. This is a Markov Modulated Poisson Process (MMPP). The state space for this Markov model is shown in Figure 2.5.

This is a two dimensional representing of Markov queuing model. The $x$ axis is the state for the first virtual server, and the $y$ axis is the state for the second virtual server. Figure 2.5 reveals the mean service rate is $\mu$ if only one of the servers is active, while the mean service rate is $\frac{\mu}{2}$ when two servers are both active. In this case the authors in [1] derived a heuristic algorithm which iteratively tries to find an accurate amount for state probabilities and performance metrics.

Although the third model expresses the virtual server behaviour more realistically, it is still needed to present a model for the case of larger number of virtual servers over a single physical machine. The model will be much more complex in the case of having more than two virtual servers on a single physical machine. Besides, it is needed to make it clear that the server capacity is assigned to different virtual nodes and it is not sharable, or the idle capacity can be shared with the other active virtual networks. So, in the case the virtual servers act isolated and independent of 
each other, it is possible to model the server with $\mathrm{M} / \mathrm{M} / \mathrm{k}$ queuing model based on the number of virtual cores we have on the actual physical machine. Consequently, further studies are essential to design a model that can present a large number of virtual servers on a single physical machine, with different constraints like memory, network processing rate, etc.

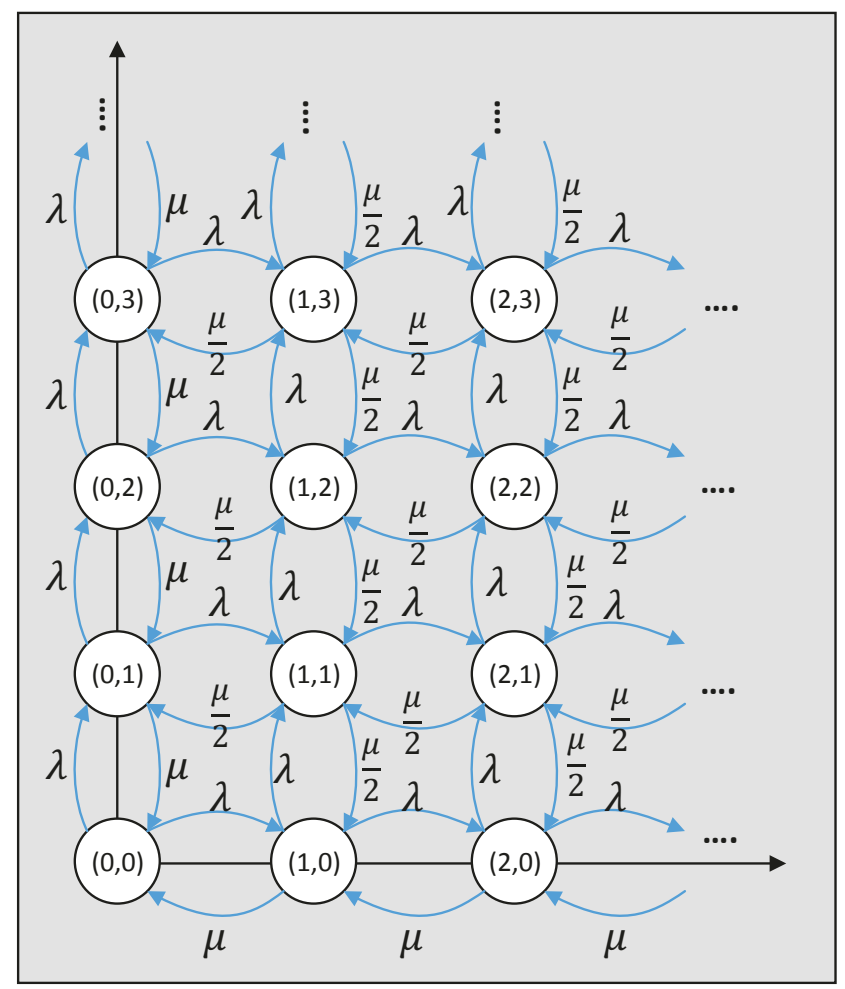

Figure 2.5: Two dimensional Markov-Chain model for two virtualized severs on a single physical server [1]

\subsection{Energy-Saving for VNEs}

As it is explained in Section 1.1, the ICT's energy consumption is increasing rapidly by a rate of $6 \%$ a year. It is anticipated that the size of the Internet network doubles every 5.32 years [2]. Therefore, researchers in recent years studied different energy-saving solutions for variety of communication network environments. On the other hand, one of the promising new technologies that is proposed for a network environment is virtualization. A virtualized network environment was reviewed in Sections 2.1 and 2.2. However, there are only very few works in the literature that concerned about 
energy consumption in VNEs.

In order to come up with effective energy-saving solutions, it is required to find out the sources of energy consumption in the focused network infrastructure. In VNEs, virtual networks are mapped on a physical network. Consequently, the main energy consumption of VNE is coming from the underlay physical network that virtual networks are embedded onto. A VNE does not change power models provided for physical networks, because the the substrate network is still the main energy consumer in its architecture. But, mapping virtual networks onto a physical network might increase the substrate network's energy consumption.

Therefore, in this section, we study the energy consumption sources in wired and data center networks as the more probable physical network basis for VNEs. In this regard, physical nodes and links are two main elements that play a key role in wired as well as data center network infrastructures. We study several power models for physical nodes and links. Besides, we review the available researches that studied the energy optimization problem for VNEs.

To understand power and energy management mechanisms, it is necessary to clearly define them. Power is the rate at which a system performs a work, and it is measured with the unit of Watt that is Joules/Second. Energy is the amount of work has been performed by a system in a period of time, and it is measured with the unit of Watt-hour. In this regard, power and energy-saving methods are closely connected [10].

\subsubsection{Node Power Models}

One of the most important network elements in every wired network is a node. A node in a wired network can be a switch, a router, or a server. Virtual nodes are mapped through a VNE embedding process onto the best of matched physical node candidates. In terms of the node energy consumption, the physical nodes are the one that drains the energy. However, embedding a virtual node onto a physical node might add extra energy consumption to the substrate node. The power consumption of substrate nodes can be modelled in different ways. Several papers studied power consumption models of physical network nodes. In this section, we review some of the models that formulate the physical node power consumption, and also the extra power consumption caused by embedding a VN request.

It is possible to formulate three types of physical node power models based on 
the traffic load [56]. The first type defines a constant amount of power consumption $\tilde{p}^{m}\left(v_{s}^{i}\right)$ for an active physical node $v_{s}^{i}$, regardless of its traffic load. $\tilde{p}^{m}\left(v_{s}^{i}\right)$ is the maximum power consumption of a substrate node $v_{s}^{i}$. This is the common case in today's networks [56]. We call this type of node power model, Fixed node power model. The second type, defines a base power $\tilde{p}^{b}\left(v_{s}^{i}\right)$ that keeps a node $v_{s}^{i}$ operational. However, different from the previous type, the node actual power consumption varies linearly between the base power consumption $\tilde{p}^{b}\left(v_{s}^{i}\right)$ (when there is no traffic in the node) and the maximum power consumption $\tilde{p}^{m}\left(v_{s}^{i}\right)$ (when the node is fully utilized). We call this type of node power model, Semi-Proportional node power model. In the third type, the node actual power consumption is fully proportional to the node traffic load. The base power consumption $\tilde{p}^{b}\left(v_{s}^{i}\right)$ is assumed as 0 , and the node actual power consumption varies linearly between 0 and $\tilde{p}^{m}\left(v_{s}^{i}\right)$, only based on the node's traffic load. This type is called Fully-Proportional node power model. Figure 2.6 shows these three power models.

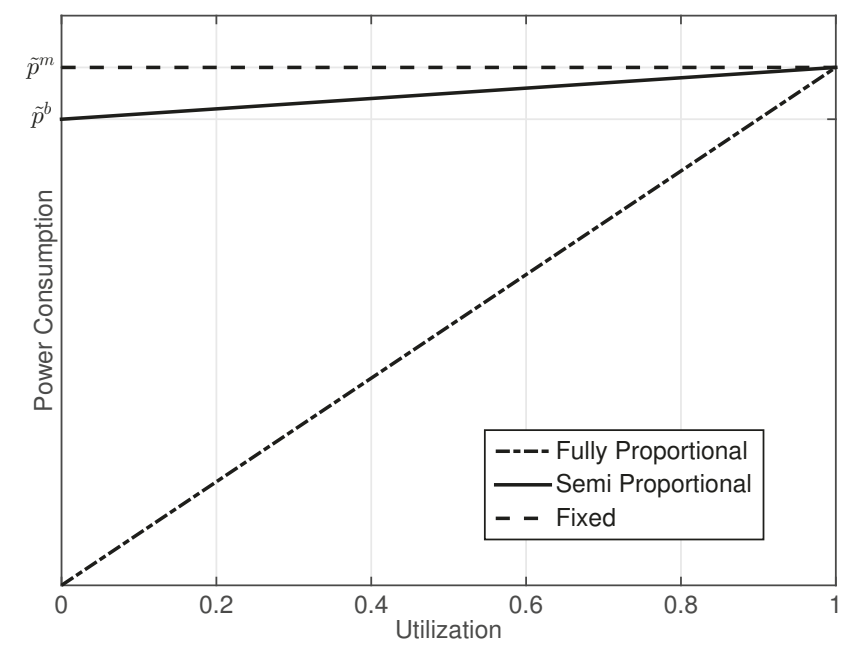

Figure 2.6: Three types of power models

Equation 2.45 defines Fixed node power model. If a physical node $v_{s}^{i}$ is active, its actual power consumption $\tilde{p}\left(v_{s}^{i}\right)$ is a constant amount, regardless of its traffic load. $\alpha\left(v_{s}^{i}\right)$ is 1 if $v_{s}^{i}$ is active, otherwise it is 0. Equation 2.46 formulates Semi-Proportional node power model, where an active physical node consumes a base amount of power $\tilde{p}^{b}\left(v_{s}^{i}\right)$, as well as the traffic-related amount of power $\frac{r\left(v_{s}^{i}\right)}{C_{b}\left(v_{s}^{i}\right)}\left(\tilde{p}^{m}\left(v_{s}^{i}\right)-\tilde{p}^{b}\left(v_{s}^{i}\right)\right)$. Where, $r\left(v_{s}^{i}\right)$ is the traffic load in $v_{s}^{i}$, and $C_{b}\left(v_{s}^{i}\right)$ is its bandwidth capacity. In addition, 
Equation 2.47 expresses the Fully-Proportional node power model, where the node actual power consumption is fully dependent on its traffic load.

$$
\begin{gathered}
\tilde{p}\left(v_{s}^{i}\right)=\alpha\left(v_{s}^{i}\right) \tilde{p}^{m}\left(v_{s}^{i}\right) \\
\tilde{p}\left(v_{s}^{i}\right)=\alpha\left(v_{s}^{i}\right) \tilde{p}^{b}\left(v_{s}^{i}\right)+\frac{r\left(v_{s}^{i}\right)}{C_{b}\left(v_{s}^{i}\right)}\left(\tilde{p}^{m}\left(v_{s}^{i}\right)-\tilde{p}^{b}\left(v_{s}^{i}\right)\right) \\
\tilde{p}\left(v_{s}^{i}\right)=\alpha\left(v_{s}^{i}\right) \frac{r\left(v_{s}^{i}\right)}{C_{b}\left(v_{s}^{i}\right)}\left(\tilde{p}^{m}\left(v_{s}^{i}\right)-\tilde{p}^{b}\left(v_{s}^{i}\right)\right)
\end{gathered}
$$

Authors in $[56,57]$ defined $\tilde{p}^{b}\left(v_{s}^{i}\right)$ and $\tilde{p}^{m}\left(v_{s}^{i}\right)$ for a physical node $v_{s}^{i}$ that is a switch or router. This is shown in Equation 2.48, and Equation 2.49.

$$
\begin{gathered}
\tilde{p}^{b}\left(v_{s}^{i}\right)=0.85 C_{b}^{\frac{2}{3}}\left(v_{s}^{i}\right) \\
\tilde{p}^{m}\left(v_{s}^{i}\right)=C_{b}^{\frac{2}{3}}\left(v_{s}^{i}\right)
\end{gathered}
$$

Fan and others in [58] derived a linear and a non-linear power model for a server, based on measurements. They believe the power consumption of a server as a physical node $v_{s}^{i}$ can be approximated linearly by Equation 2.50. Where, $U_{c}\left(v_{s}^{i}\right)$ is the CPU utilization of a physical node $v_{s}^{i}$.

$$
\tilde{p}\left(v_{s}^{i}\right)=\alpha\left(v_{s}^{i}\right) \tilde{p}^{b}\left(v_{s}^{i}\right)+U_{c}\left(v_{s}^{i}\right)\left(\tilde{p}^{m}\left(v_{s}^{i}\right)-\tilde{p}^{b}\left(v_{s}^{i}\right)\right)
$$

They also provided a non-linear power model that accurately defines a server's power consumption. This is shown in Equation 2.51. $\psi$ is a tuneable weight that can be found through calibration experiments for each set of deployed servers. Such a calibration process is suggested in [59]. They assumed $\psi=1.4$.

$$
\tilde{p}\left(v_{s}^{i}\right)=\alpha\left(v_{s}^{i}\right) \tilde{p}^{b}\left(v_{s}^{i}\right)+\left(2 U_{c}\left(v_{s}^{i}\right)-U_{c}\left(v_{s}^{i}\right)^{\psi}\right)\left(\tilde{p}^{m}\left(v_{s}^{i}\right)-\tilde{p}^{b}\left(v_{s}^{i}\right)\right)
$$

Their extensive experiments on thousands of servers reveals Equation 2.50 defines the power consumption of a server by $5 \%$ error, while Equation 2.51 has only $1 \%$ error.

Andres Fischer in [60] formulated the power consumption of a substrate network in a VNE. They believe the main power consumption in a VNE is coming from physical 
nodes. It is assumed a substrate node only consumes power when either a virtual node is mapped onto it, or it is a part of a path for a mapped virtual link. The proposed model in [60] defines the total substrate network power consumption as follows:

$$
\tilde{p}\left(G_{s}\right)=\sum_{i \in V_{s}} \alpha\left(v_{s}^{i}\right) \tilde{p}\left(v_{s}^{i}\right)
$$

This model simply sums the power consumption $\tilde{p}\left(v_{s}^{i}\right)$ of every active physical node. They claim this model is realistic and feasible to calculate [60]. Nevertheless, this power model does not explain a single node's power model, and how to calculate $\tilde{p}\left(v_{s}^{i}\right)$.

Joseph Chabarek in [61] measured the power demand for two widely Cisco routers over different configuration and created a power consumption model for routers. This general model for the power consumption of an active router as a physical node $v_{s}^{i}$ is shown in Equation 2.53.

$$
\tilde{p}\left(v_{s}^{i}\right)=\tilde{p}^{b}\left(v_{s}^{i}\right)+\sum_{j=1}^{\left|\Upsilon^{i}\right|}\left(\hbar\left(\Upsilon^{i, j}\right)+\grave{c}\left(\Upsilon^{i, j}\right)\right)
$$

Equation 2.53 describes the power consumption of an active router, which is proportional to a chassis type of a device, the installed line cards and also a device configurations and traffic models. $\tilde{p}^{b}\left(v_{s}^{i}\right)$ stands for the base power consumption of a router's chassis, while $\left|\Upsilon^{i}\right|$ is the number of active line cards in a router $v_{s}^{i}$. $\hbar\left(\Upsilon^{i, j}\right)$ is scaling factor based on the traffic utilization over the $j$ th line card in a $v_{s}^{i}$, while $\grave{c}\left(\Upsilon^{i, j}\right)$ shows the cost of the $j$ th line card in a base configuration of a $v_{s}^{i}$. The cost of traffic is dependent on the configuration of the router and the amount of traffic [61].

This model is based on multi-chassis systems. The architecture for a multi-chassis router consists of several line cards connected to a non-blocking scalable switch fabric chassis [61]. Therefore, by having more traffic over a router, we need more line cards in order to handle the new traffic load. Considering Equation 2.53, by increasing the traffic load, and therefore growing the number of line cards, the router power consumption will be increased as well. But, still the power is mainly consumed by the chassis. Hence, the number of active routers is an important metric in terms of total node power consumption of a network. Nevertheless, the authors in [61] do not clarify how to find $\grave{c}\left(\Upsilon^{i, j}\right)$ and what does it mean to have the line card cost in the router power model. 
Bin Wang and others in [62] derived a generalized node power consumption model for embedding a virtual network onto a substrate network in a VNE. They assumed that a physical node is turned off only when all its network ports, or connected links are turned off. Besides, if a node is unused, then it is simply turned off and has zero power consumption. In terms of the node power consumption, they defined a node energy consumption model $\tilde{p}\left(V_{n}\right)$ for accepting a $V_{n}$ in unit time as:

$$
\tilde{p}\left(V_{n}\right)=\sum_{i \in V_{s}} \tilde{p}\left(v_{s}^{i}\right)\left(\check{\alpha}\left(v_{s}^{i}\right)-\hat{\alpha}\left(v_{s}^{i}\right)\right)+\sum_{i \in V_{s}} \tilde{p}^{c}\left(v_{s}^{i}\right) C_{c}\left(v_{s}^{i}\right)
$$

Here, a physical node can be a router or a server. The first term in Equation 2.54 is the unit-time power consumption associated with those inactive physical nodes that turned on after embedding a $V_{n}$, where $\check{\alpha}\left(v_{s}^{i}\right)$ and $\hat{\alpha}\left(v_{s}^{i}\right)$ are the node status after and before the embedding, respectively. The second term in this equation is the variable unit-time power consumption that is added by the CPU. $\tilde{p}^{c}\left(v_{s}^{i}\right)$ is the power consumption of the unit CPU utilization of a $v_{s}^{i}$ in unit time, and $C_{c}\left(v_{s}^{i}\right)$ represents the CPU capacity of the substrate node.

The node power consumption for accepting a virtual network is well described in this model. It is a good idea to find out the node status before and after embedding to derive the power consumption that is added by embedding a particular VN. However, the model is weak in terms of modelling the node power consumption. It uses $\tilde{p}\left(v_{s}^{i}\right)$ as the physical node power consumption, while it is not defined how to calculate it. Besides, it adds the CPU power consumption as a separate part, but it should already be included in $\tilde{p}\left(v_{s}^{i}\right)$.

Sen Su, and Zhongbao Zhang in [63] and [64] introduced a power model for a virtual node as well as a node power consumption model for embedding a VN request. They used a server power model for estimating the node power consumption in the context of virtual network embedding. Their suggested model is shown in Equation 2.55. In this model, the CPU utilization is the main contributor to the power consumption variations of a node. In the case a substrate node $v_{s}^{i}$ is active $\left(\alpha\left(v_{s}^{i}\right)=1\right)$, its power consumption is calculated based on the node's base power consumption $\tilde{p}^{b}\left(v_{s}^{i}\right)$, the maximum power when serving at the maximum capacity $\tilde{p}^{m}\left(v_{s}^{i}\right)$, and its CPU utilization $U_{c}\left(v_{s}^{i}\right)$, as follows: 


$$
\tilde{p}\left(v_{s}^{i}\right)=\left\{\begin{array}{cl}
\tilde{p}^{b}\left(v_{s}^{i}\right)+\left(\tilde{p}^{m}\left(v_{s}^{i}\right)-\tilde{p}^{b}\left(v_{s}^{i}\right)\right) U_{c}\left(v_{s}^{i}\right) & \alpha\left(v_{s}^{i}\right)=1 \\
0 & \alpha\left(v_{s}^{i}\right)=0
\end{array}\right.
$$

According to this model, the additional power consumption $\tilde{p}\left(v_{n}^{k}, v_{s}^{i}\right)$ produced by embedding a virtual node $v_{n}^{k}$ with the demanded CPU capacity of $C_{c}\left(v_{n}^{k}\right)$ onto a physical link $v_{s}^{i}$, is calculated in Equation $2.56[63,64]$.

$$
\tilde{p}\left(v_{n}^{k}, v_{s}^{i}\right)=\left\{\begin{array}{rr}
\tilde{p}^{b}\left(v_{s}^{i}\right)+\left(\tilde{p}^{m}\left(v_{s}^{i}\right)-\tilde{p}^{b}\left(v_{s}^{i}\right)\right) C_{c}\left(v_{n}^{k}\right) & \hat{\alpha}\left(v_{s}^{i}\right)=0 \\
\left(\tilde{p}^{m}\left(v_{s}^{i}\right)-\tilde{p}^{b}\left(v_{s}^{i}\right)\right) C_{c}\left(v_{n}^{k}\right) & \hat{\alpha}\left(v_{s}^{i}\right)=1
\end{array}\right.
$$

Equation 2.56 shows in the case a virtual node $v_{n}^{k}$ is mapped onto a substrate node $v_{s}^{i}$ which is already active $\left(\hat{\alpha}\left(v_{s}^{i}\right)=1\right)$, the additional power consumption is only related to the demanded CPU capacity. However, in the case a substrate node is inactive before the embedding process $\left(\hat{\alpha}\left(v_{s}^{i}\right)=0\right)$, the additional power consumption includes the node base power as well as the required power to handle the demanded CPU capacity. Consequently, the additional node power consumption $\tilde{p}\left(V_{n}\right)$ for embedding nodes $V_{n}$ of the $n$th virtual network onto a substrate network, is proportional to the number of physical nodes needed to be powered on from the off state [64]. This is proved in Equation 2.57.

$$
\begin{aligned}
\tilde{p}\left(V_{n}\right) & =\sum_{k \in V_{n}} \tilde{p}\left(v_{n}^{k}, v_{s}\right) \\
& =\sum_{k \in V_{n}}\left(\sum_{i \in V_{s}} \phi\left(v_{n}^{k}, v_{s}^{i}\right)\left(1-\hat{\alpha}\left(v_{s}^{i}\right)\right) \tilde{p}^{b}\left(v_{s}^{i}\right)+\left(\tilde{p}^{m}\left(v_{s}^{i}\right)-\tilde{p}^{b}\left(v_{s}^{i}\right)\right) C_{c}\left(v_{n}^{k}\right)\right) \\
& =\underbrace{\sum_{k \in V_{n}} \sum_{i \in V_{s}} \phi\left(v_{n}^{k}, v_{s}^{i}\right)\left(1-\hat{\alpha}\left(v_{s}^{i}\right)\right) \tilde{p}^{b}\left(v_{s}^{i}\right)}+\left(\tilde{p}^{m}\left(v_{s}^{i}\right)-\tilde{p}^{b}\left(v_{s}^{i}\right)\right) \sum_{k \in V_{n}} C_{c}\left(v_{n}^{k}\right)
\end{aligned}
$$

Where $\phi\left(v_{n}^{k}, v_{s}^{i}\right)$ is a binary variable that is equal to 1 if a virtual node $v_{n}^{k}$ is mapped onto a substrate node $v_{s}^{i}$, otherwise $\phi\left(v_{n}^{k}, v_{s}^{i}\right)$ is 0 .

This model formulates the embedding power consumption more realistically, in comparison to Equation 2.54, because Equation 2.56 considers different amounts of 
power consumption for different CPU loads, while this was not included in Equation 2.54. Nevertheless, this model similar to Equation 2.54 only acknowledges the requested $\mathrm{CPU}$ capacity in terms of power consumption, while the customers might only concern about other node attributes like memory. This should be included in a node power model for VNEs.

In summary, based on the cited studies, three main factors impact the node power consumption in VNEs. The main factor is the node base power consumption which is needed to keep a physical node operational. The second factor is traffic. The third factor is the required processor capacity or the CPU utilization. Hence, the best energy-saving mechanism is deactivating a device, so it consumes no power. It is also probable to manage the traffic load or the processing load of physical nodes to optimize their power consumption.

\subsubsection{Link Power Models}

A physical link is another important element in wired and data center networks. Every physical node is connected to another physical node through physical links. The virtual links are mapped onto the physical paths through an embedding process. A virtual link might be mapped onto a single physical link or a series of physical links as a path. In the case a virtual link is embedded over a physical path, there are intermediate physical nodes which are responsible only for packet forwarding. It is essential to study a physical link power model in order to find the factors affect its power consumption. In this section, we review some available physical link power models, and also link embedding power models for VNEs.

Three types of linear power models can be used to define the physical link power consumption, according to [56]. The first type considers a fixed amount of power consumption $\tilde{p}^{m}\left(l_{s}^{i, j}\right)$, that is the maximum power consumption of a physical link $l_{s}^{i, j}$ for different ranges of physical link capacity. According to this type, the actual link power consumption is not affected by the actual traffic load in the link. Therefore, a physical link $l_{s}^{i, j}$ consumes a fix amount of power $\left(\tilde{p}^{m}\left(l_{s}^{i, j}\right)\right)$, if it is active, regardless of its traffic load. This is the common case in today's networks [56]. We call this type Fixed link power model. The second type assumes a base amount of power $\tilde{p}^{b}\left(l_{s}^{i, j}\right)$ that keeps a physical link $l_{s}^{i, j}$ operational. However, despite the previous type, the actual

link power consumption varies linearly, between the base power consumption $\tilde{p}^{b}\left(l_{s}^{i, j}\right)$ (when there is no traffic in the link), and the maximum power consumption $\tilde{p}^{m}\left(l_{s}^{i, j}\right)$ 
(when the link is fully utilized). In this study, this power model type is called SemiProportional link power model. In the third type of link power models, the actual link power consumption is fully proportional to the traffic load. The base power $\tilde{p}^{b}\left(l_{s}^{i, j}\right)$ is assumed as 0 , and the actual power consumption varies linearly between 0 and $\tilde{p}^{m}\left(l_{s}^{i, j}\right)$, only based on the link's traffic load. This type is called Fully-Proportional link power model in this study. Figure 2.6 shows different types of power models.

In this regard, according to [56], three types of link power models can be formulated as follows:

$$
\begin{gathered}
\tilde{p}\left(l_{s}^{i, j}\right)=\alpha\left(l_{s}^{i, j}\right) \tilde{p}^{m}\left(l_{s}^{i, j}\right) \\
\tilde{p}\left(l_{s}^{i, j}\right)=\alpha\left(l_{s}^{i, j}\right) \tilde{p}^{b}\left(l_{s}^{i, j}\right)+\frac{r\left(l_{s}^{i, j}\right)}{C_{b}\left(l_{s}^{i, j}\right)}\left(\tilde{p}^{m}\left(l_{s}^{i, j}\right)-\tilde{p}^{b}\left(l_{s}^{i, j}\right)\right) \\
\tilde{p}\left(l_{s}^{i, j}\right)=\alpha\left(l_{s}^{i, j}\right) \frac{r\left(l_{s}^{i, j}\right)}{C_{b}\left(l_{s}^{i, j}\right)}\left(\tilde{p}^{m}\left(l_{s}^{i, j}\right)-\tilde{p}^{b}\left(l_{s}^{i, j}\right)\right)
\end{gathered}
$$

$\alpha\left(l_{s}^{i, j}\right)$ denotes the status of an $l_{s}^{i, j}$. When the link is active $\alpha\left(l_{s}^{i, j}\right)=1$, otherwise $\alpha\left(l_{s}^{i, j}\right)=0$. Equation 2.58 is Fixed link power model that defines a constant amount of power consumption for a physical link. Nonetheless, Equation 2.59 is Semi-Proportional link power model. In the case a link $l_{s}^{i, j}$ is active $\left(\alpha\left(l_{s}^{i, j}\right)=1\right)$, it consumes $\tilde{p}^{b}\left(l_{s}^{i, j}\right)$. In addition, the traffic load in the link increases its power consumption linearly. However, Equation 2.60 is Fully-Proportional link power model. In this model, the physical link power consumption is varying linearly only based on its utilization.

$\tilde{p}^{b}\left(l_{s}^{i, j}\right)$, and $\tilde{p}^{m}\left(l_{s}^{i, j}\right)$ are normally defined for different ranges of bandwidth capacity, and according to the link's length and the type of the cable. Table 2.2 summarizes some examples for $\tilde{p}^{b}\left(l_{s}^{i, j}\right)$ and $\tilde{p}^{m}\left(l_{s}^{i, j}\right)$.

Table 2.2: Parameters of physical link power consumption

\begin{tabular}{|l|c|c|c|}
\hline$C_{b}\left(l_{s}^{i, j}\right)$ & $\tilde{p}^{b}\left(l_{s}^{i, j}\right)[$ Watt] & $\tilde{p}^{m}\left(l_{s}^{i, j}\right)[$ Watt] & Ref. \\
\hline 0-100 Mbps & 0.48 & 0.48 & {$[65][66]$} \\
\hline 100-600 Mbps & 0.9 & 1.00 & {$[65][66]$} \\
\hline 600-1000 Mbps & 1.70 & 2.00 & {$[67]$} \\
\hline
\end{tabular}


In addition, Spyridon, Stevon and Lisa, from Bell Laboratories, also adopted a simple float model for the physical link power consumption in [68]. The model consists of a fixed parameter named base power ratio, which determines the required amount of power consumption to keep the link operational, as well as another parameter which is dependent on traffic. The power model of a physical link $l_{s}^{i, j}$ is shown in Equation 2.61. $\zeta$ is representing the link base power ratio. $C_{b}\left(l_{s}^{i, j}\right)$ stands for the bandwidth capacity of a physical link $l_{s}^{i, j}$. The actual substrate link power consumption $\tilde{p}\left(l_{s}^{i, j}\right)$ changes according to loaded traffic $r\left(l_{s}^{i, j}\right)$ in the link, between $\zeta C_{b}\left(l_{s}^{i, j}\right)$ and $C_{b}\left(l_{s}^{i, j}\right)$ for $r\left(l_{s}^{i, j}\right)=0$ and $r\left(l_{s}^{i, j}\right)=C_{b}\left(l_{s}^{i, j}\right)$, respectively.

$$
\tilde{p}\left(l_{s}^{i, j}\right)=\left\{\begin{array}{cc}
0 & r\left(l_{s}^{i, j}\right)=0 \\
\zeta C_{b}\left(l_{s}^{i, j}\right)+(1-\zeta) r\left(l_{s}^{i, j}\right) & 0<r\left(l_{s}^{i, j}\right) \leq C_{b}\left(l_{s}^{i, j}\right)
\end{array}\right.
$$

Consequently, based on the model shown in Equation 2.61, two factors have impact on the total power consumption of a physical link, the base power ratio $\zeta$, and the data traffic load $r\left(l_{s}^{i, j}\right)$ in the link. Setting $\zeta$ to $100 \%$ leads to have a Fixed link power model that sets a constant link power consumption, regardless of traffic load. On the other hand, setting $\zeta$ to $0 \%$ gives a Fully-Proportional power model that depends only on traffic load. Any other base power ratio $\zeta$ brings a Semi-Proportional link power model. Thus, by adjusting $\zeta$ we are able to increase, or decrease traffic load impact on physical link power consumption.

We discussed different power models for a physical link. However, it is also required to study the impact of a virtual link embedding on the physical link power consumption. Therefore, we review some available power models for a VNE link embedding, in the followings:

Bin Wang, in the same paper [62] that presented the node embedding power model, also proposed a model for the power consumption of accepting an $E_{n}$ onto a substrate network. The model is shown in in Equation 2.62.

$$
\tilde{p}\left(E_{n}\right)=\sum_{(i, j) \in E_{s}} \tilde{p}\left(l_{s}^{i, j}\right)\left(\check{\alpha}\left(l_{s}^{i, j}\right)-\hat{\alpha}\left(l_{s}^{i, j}\right)\right)
$$

This model considers the link states after $\check{\alpha}\left(l_{s}^{i, j}\right)$ and before $\hat{\alpha}\left(l_{s}^{i, j}\right)$ an embedding process to find which links are turned on by embedding a virtual network. In the case a physical link is active, $\check{\alpha}\left(l_{s}^{i, j}\right) \backslash \hat{\alpha}\left(l_{s}^{i, j}\right)$ is 1 , otherwise it is 0 . Therefore, it is possible to calculate the extra power consumption added by embedding the links. Considering 
both node (Equation 2.54) and link (Equation 2.62) power models, we can formulate the embedding power consumption of a $G_{n}$ by Equation 2.63.

$$
\tilde{p}\left(G_{n}\right)=\sum_{i \in V_{s}} \tilde{p}\left(v_{s}^{i}\right)\left(\check{\alpha}_{v_{s}^{i}}-\hat{\alpha}_{v_{s}^{i}}\right)+\sum_{i \in V_{s}} \tilde{p}^{c}\left(v_{s}^{i}\right) C_{c}\left(v_{s}^{i}\right)+\sum_{(i, j) \in E_{s}} \tilde{p}\left(l_{s}^{i, j}\right)\left(\check{\alpha}\left(l_{s}^{i, j}\right)-\hat{\alpha}\left(l_{s}^{i, j}\right)\right)
$$

As mentioned in the previous section, this model does not explain the method of finding the power consumption of a single node or link, while it calculates the embedding power consumption based on them.

Sen Su, and Zhongbao Zhang at [63] and [64] also presented a virtual link power model and a virtual link embedding power model. It is a common belief that dedicated offload engines which send and received packets will be widely deployed in VNEs [64]. The advantages of these engines are the high packet processing rate and the low processing latency [64]. The authors in [64] consider the engine power consumption $\tilde{p}^{e}\left(v_{s}^{i}\right)$ as a constant whether the ports are idle or carrying full speed traffic. In a VNE embedding process, a virtual link might be embedded onto a single physical link or sometimes may span a number of physical links as a path. In the case a virtual link is mapped onto a path, the nodes other than the two ends are called intermediates nodes. The intermediate nodes are solely responsible for packet forwarding [64]. Authors of [63] and [64] believe the link power consumption comes only from the nodes on the path. Therefore, they modelled the virtual link embedding power consumption by the power consumed at the physical nodes which the virtual links is mapped onto. Hence, the link embedding power consumption of sending and receiving packets at an end node is formulated in Equation 2.64. However, this amount at an intermediate node is calculated at Equation 2.65.

$$
\begin{gathered}
\tilde{p}\left(l_{n}^{a, b}, v_{s}^{i}\right)=\left\{\begin{array}{cc}
\tilde{p}^{e}\left(v_{s}^{i}\right) & \hat{\alpha}\left(v_{s}^{i}\right)=0 \\
0 & \hat{\alpha}\left(v_{s}^{i}\right)=1
\end{array}\right. \\
\tilde{p}\left(l_{n}^{a, b}, v_{s}^{i}\right)=\left\{\begin{array}{cc}
\tilde{p}^{b}\left(v_{s}^{i}\right)+\tilde{p}^{e}\left(v_{s}^{i}\right) & \hat{\alpha}\left(v_{s}^{i}\right)=0 \\
0 & \hat{\alpha}\left(v_{s}^{i}\right)=1
\end{array}\right.
\end{gathered}
$$

$\tilde{p}\left(l_{n}^{a, b}, v_{s}^{i}\right)$ refers to the additional power consumption when embedding an $l_{n}^{a, b}$ causes activating a $v_{s}^{i}$. Hence, according to [64] a link embedding will not add any power consumption in the case no new physical node is got activated. Consequently, 
it is possible to prove the additional link power consumption caused by a VN embedding process, is proportional to both the number of end nodes and intermediate nodes, which are turned on from off state. This is proved mathematically in Equation 2.66 .

$$
\begin{aligned}
\tilde{p}\left(L_{n}\right) & =\sum_{(a, b) \in E_{n}} \tilde{p}\left(l_{n}^{a, b}, v_{s}\right) \\
& =\underbrace{\sum_{k \in V_{n}} \sum_{i \in V_{s}} \phi\left(v_{n}^{k}, v_{s}^{i}\right)\left(1-\hat{\alpha}\left(v_{s}^{i}\right)\right)}_{\text {number of working nodes needed to be powered on }} \tilde{p}^{e}\left(v_{s}^{i}\right) \\
& +\underbrace{\sum_{i \in V_{s}} \min \left(1, \sum_{\left\{j \mid(i, j) \in E_{s}\right\}} \phi\left(l_{n}^{a, b}, l_{s}^{i, j}\right)\right)\left(1-\hat{\alpha}\left(v_{s}^{i}\right)\right)}_{\text {number of intermediate nodes needed to be powered on }}\left(\tilde{p}^{b}\left(v_{s}^{i}\right)+\tilde{p}^{e}\left(v_{s}^{i}\right)\right)
\end{aligned}
$$

Where, $\phi\left(v_{n}^{k}, v_{s}^{i}\right)$ is 1 if a $v_{n}^{k}$ is allocated in a $v_{s}^{i}$, otherwise it is 0. Similarly, $\phi\left(l_{n}^{a, b}, l_{s}^{i, j}\right)$ is 1 if an $l_{n}^{a, b}$ is allocated in an $l_{s}^{i, j}$, otherwise it is 0 .

Nevertheless, this is not a true reflection of the physical link power consumption. According to the other cited studies like [68], a physical link itself, other than packet processor engines, consumes the power. Besides, the traffic load on a link may change the amount of power which is consumed by the physical link.

In summary, various types of link power models define the physical link power consumption, differently. The common link power model that describes the energy consumption of today's networks is Fixed link power model, that define a constant amount of power for an active physical link. However, it is expected physical links are get modified in the future, so their power consumption is proportional to their traffic load. Semi-Proportional and Full Proportional power models stands for this concept. In addition, a virtual network embedding adds extra power consumption to an underlay physical network. The extra power consumption added by embedding a virtual network is proportional to the number of end and intermediate nodes which are turned on to handle a virtual link's traffic. 


\subsubsection{Existing Energy-Saving Solutions for VNEs}

There are several papers in the literature discussing virtualized network environments from different aspects, and also there are several other studies which addressed the energy-efficient networks. Nevertheless, to the best of our knowledge, there are only few initial studies which have analyzed and reviewed the energy consumption issue specifically for VNE. We review them in the followings:

First, four papers $[60,62,64,69]$ tried to save energy in a VNE by making an embedding procedure energy-aware. An embedding procedure in VNEs maps a requested virtual network topology, coming from customers, onto a substrate network based on customers and providers' constraints. Therefore, in order to have an energy-efficient VNE, it might be feasible to map the virtual network demands onto a substrate network taking into account the physical network' power consumption.

Andres Fischer and others in [60] suggested an energy-efficient embedding algorithm. They modified the links' weights in their embedding routing algorithm. The new links' weights are calculated based on their power consumption. Hence, their embedding algorithm concerns about physical links power consumption and attemps to map the requested virtual links onto the less power consuming substrate paths.

Authors in [64] proposed an energy-aware VNE embedding algorithm by consolidating virtual networks to the smallest number of substrate nodes and powering down unused substrate nodes. They formulated an ILP with an objective of minimizing the number of active substrate nodes.

Bin Wang and others also in [62] offered an energy-aware VNE embedding algorithm. They have first proposed a power model for a VNE and then based on the suggested power model, formulated a mixed integer program while its objective is minimizing the energy consumption added by embedding of a virtual network request.

In a similar approach as [64], Juan Felipe Botero and his co-authors in [69] attempted to minimize the power consumption of a VNE by means of resource consolidation in an embedding procedure. They also formulated a mixed integer program in order to find the optimal energy-efficient embedding.

Although modifying the embedding algorithms in order to achieve an energyefficient VNE is an effective approach, it suffers from a major difficulty. When VNE embedding algorithms are modified to map the resources energy-wise, several extra constraints will be added to the embedding procedure. Accordingly, the embedding algorithm has a smaller set of physical node and link candidates to choose from. This 
decreases the network's admittance ratio for new virtual network requests, which is not cost efficient for the providers. The main economic objective of providers is to reject the minimum number of virtual network requests. Thus, these solutions are not profitable for them in long term.

Some other papers studied heuristic algorithms that modify the already mapped VNs. Juan Fleipe Botero in [17] offered a heuristic algorithm that reconfigures the mapping of accepted VNs at each embedding phase to save the energy. This approach has the same problem of energy-efficient embedding methods. Because reconfiguring the mapping of accepted VNs at each embedding phase for their life time, still might make capacity bottlenecks that decrease the network admittance rate for new VNs. Besides, it suffers from multiple other weaknesses. First, it does not consider the actual load on the network. The traffic load in a VNE is changing over the time and most of the allocated capacities are wasted in off-peak hours, because the network elements consume the same energy as the peak time. Nevertheless, the algorithm presented in [17] reconfigures the virtual networks, after embedding procedure, to re-allocate the virtual links peak demands. However, it is not efficient to re-allocate only according to the peak traffic demands. Second, their heuristic attempts to re-allocate virtual links while it does not consider physical links. Nonetheless, the energy is consumed by physical links, and not by each virtual link. Therefore, their algorithm's objective is not efficient. Third, the heuristic assumes that each virtual link is allocated in a single physical link. However, the virtual links might get mapped onto a physical path. Fourth, the alternative paths are assumed to be pre-calculated, but this makes the algorithm less effective in a dynamic network environment like a VNE. Finally, their reconfiguration problem is not formulated in a mathematical format or an optimization program.

An off-line heuristic reconfiguration algorithm is proposed in our previous work [70]. The algorithm attempts to maximize the number of physical links in sleep mode during the off-peak period of a VNE. It reroutes the off-peak traffic of already embedded virtual links, to other already allocated traffic capacities. It does not change the mapping of VNs. Assuming a fixed VN mapping prevents us from rerouting a VN's off-peak traffic to substrate links that no traffic capacity is allocated in them to that particular VN. This decreases the level of energy we can save.

Authors in [16] suggested a method to move the already embedded virtual machines onto servers, to other servers. Their solution is run over time periodically, to 
consolidate the VMs. Nevertheless, moving allocated VMs and setting the servers into sleep mode is expensive, if it is not impossible, due to two reasons. First, normally a large amount of data is distributed over a large number of servers, and it is not profitable/possible for the providers to move data of a server to another one. Second, waking up servers from the sleep mode (in the case of unexpected demand, or returning to peak time), imposes hundreds of milliseconds delay to the tasks that might violate Service Level Objectives (SLOs) [71]. Besides, their solution does not enable the providers to adjust the level of the reconfiguration, and control the possible traffic disruptions.

In the case of having a fixed mapping for allocated virtual nodes, the problem might seem similar to the classic routing problems for a multi-layer network design. For example, Chuansheng Xin and his co-authors in [72] proposed a mapping method to design a static virtual topology for Wavelength-Division Multiplexing (WDM) optical networks. They pre-compute possible routes between the source and destination of every requested traffic demand, and then their ILP chooses the best routes according to their objective. [73], [72], and the other existing approaches for energy-efficient routing problem, e.g. [74,75], are mapping methods. They route every requested traffic demand, between fixed nodes. But, energy-aware routing of every traffic demand is quite different from locally reconfiguring the mapping of VNs and simultaneously considering the possible traffic disruptions that might happen during the reconfiguration.

To the best of our knowledge there are few papers that studied a classic energyefficient reconfiguration problem and considered the possible traffic interruptions. Authors in [76] proposed an MILP that reroutes off-peak traffic in order to minimize the energy consumption during the off-peak period. However, their approach, similar to [70] and [77], assumes a fixed VN mapping to decrease the possible traffic disruptions. But, as it is discussed, assuming a fixed VN mapping reduces the level of energy-saving. Furthermore, they do not provide a tool, so the providers can adjust the level of the reconfiguration.

Similarly, authors in [78] suggested an MILP to reroute the off-peak traffic to save the energy. Different from previous papers [70,76,77], the method in [78] first pre-computes static mappings for VNs, according to their off-peak load. They do no consider energy consumption at this step. Then, the MILP reroutes the off-peak traffic of every virtual link to the pre-computed paths, to save energy. They do not 
let the MILP to modify the mapping of virtual links, in order to reduce the possible traffic interruptions and decrease program's complexity. However, pre-computing offpeak mappings and then searching in them to save energy do not provide the most optimum result, because it is possible to reroute traffic only to the substrate links in the pre-computed mappings. Besides, rerouting the off-peak traffic of every virtual link is not a good practice, while the providers are not able to control the possible traffic interruptions. 


\section{Chapter 3}

\section{Off-Peak Energy Optimization for Links in a VNE}

\subsection{Introduction}

In fact, virtual networks' traffic loads change over time. Virtual networks might be highly utilized during a period of time (the peak time, e.g. day hours), while they are under-utilized during another notable period of time (the off-peak time, e.g. night hours). Traffic variations in virtual networks correspondingly change a substrate network's utilization. The reports for 40 North American and 25 European network providers reveal $60 \%$ difference between the peak and the minimum off-peak traffic rates over their substrate network [78]. However, today's substrate networks are provisioned to support the VNs' peak time traffic demands, with some additional over-provisioning accommodating unexpected traffic rates [78]. A substrate network's elements are always switched on, neglecting the traffic behaviour.

There could be two cases in regard to determining the off-peak time period of a substrate network and its traffic demands in that period. First, a network provider has fixed virtual network customers for a specific period of time, e.g. a week. In this case, the provider could determine the off-peak time period of the substrate network and its off-peak traffic demands in that time window, through given information by each virtual network customer. Second, the network provider has dynamic virtual network customers, so virtual network requests are coming over time. In this case, the provider could predict the traffic load for a future period of time by the network traffic prediction techniques, e.g. [79,80]. The traffic prediction techniques normally estimate the future traffic by looking at the current traffic state. Accordingly, the 
providers could determine the off-peak period in each time interval that its traffic is predicted. Note that predicting the off-peak traffic load is out of the scope of this thesis.

During the off-peak period, it is possible to reduce a VNE's energy consumption by reconfiguring the mapping of the already embedded VNs according to their decreased traffic demands. In this context, virtual networks are accepted and embedded onto a substrate network by a normal (not energy-efficient) VNE embedding process to accommodate the peak traffic behaviour. The reconfiguration technique is run during normal network operations, upon networks go from the peak period to the off-peak period, to save energy in the off-peak period. However, when the traffic loads change from a peak level to an off-peak level, some traffic flows that last in the both time periods might suffer from traffic disruptions imposed by applying the reconfiguration [78]. Besides, reconfiguring the mapping of embedded VNs may require additional signalling traffic that is necessary for notifying all the involved routers [77]. This may introduce a significant work load for the signalling controller especially when the reconfiguration attempts to make changes to a large number of nodes at the same time. Consequently, it may not be a good practice to reconfigure the mapping of every embedded virtual node/link.

The reconfiguration process needs four steps. First, it learns the peak and the offpeak periods and the associated demands. Second, it calculates the off-peak mapping. Third, at the beginning of each off-peak period, it reconfigures the mapping of all the affected VNs based on the result in the second step. Fourth, at the beginning of each peak period, it restores the original mapping.

The major cost of the reconfiguration consists of three components, collecting traffic information with each VN, calculating the off-peak mapping, and conducting the reconfiguration operation. Because the third item is supposed to be completed as fast as possible, its energy cost is insignificant. The first and the second items depend on how often they are performed. For example, if all the traffic of all VNs is roughly periodic, then the first and second items only need to be performed once whenever a new $\mathrm{VN}$ is added or a $\mathrm{VN}$ is leaving.

Due to simpler technical implementation and high potential of energy-saving in a network's links [73], this chapter is restricted to energy-saving solutions for links in a VNE. In this chapter, we discuss multiple novel energy-saving solutions that optimize a VNE's link energy consumption during the off-peak time, according to two defined 
power models. We approach the problem by reconfiguring the mapping of the already embedded virtual links.

First, we study a reconfiguration problem that re-maps every virtual link according to its off-peak traffic demand, in order to to minimize a VNE's link energy consumption during the off-peak period. This is a coarse-grained (the granularity is a virtual link) and global (it re-maps every virtual link) reconfiguration method. We study this problem as our benchmark, because it delivers the most optimum level of energy-saving for this problem. However, as it is discussed, this is not a safe approach. We formulate this method as a Mixed Integer Linear Program (MILP).

Second, we propose a novel reconfiguration methodology. We define a stress rate for a substrate link. Accordingly, a solution is proposed to minimize VNE's link energy consumption during the off-peak time. This method may set a less stressed substrate link into the sleep mode for the off-peak time. We re-map an allocated traffic capacity to a virtual link in a less stressed substrate link if we set the substrate link into the sleep mode. This is a fine-grained (the granularity is an allocated traffic capacity to a virtual link in a substrate link) and local (it does not re-map every allocated traffic capacity to virtual links) reconfiguration strategy. This method enables the providers to change the level of the reconfiguration by adjusting the stress rate's threshold, and therefore control the possible traffic interruptions. Clearly, there is a trade-off between the energy-saving level and the possible traffic interruptions. In order to study the impact of traffic splittability on energy-saving capability of our solutions, we formulate the latter method as an MILP for splittable traffic, and as a Binary Integer Linear Program (BILP) for non-splittable traffic.

The formulated optimization solutions are $\mathcal{N} \mathcal{P}$-hard and therefore they are not scalable to large network sizes. Hence, a novel heuristic algorithm is also proposed. The simulation results confirm that the heuristic algorithm can achieve closely to the optimum points set by the optimization program. While, it is scalable to large network sizes.

The related works are discussed in Section 2.3.3. In this regard, our contributions in this chapter are:

- We propose global and local optimization programs in form of ILPs for this problem.

- The solutions are formulated according to two power models. 
- A coarse-grained and a fine-grained reconfiguration method are suggested for this problem.

- We discuss how differently we should approach the problem in the case of nonsplittable traffic in comparison to splittable traffic, to have a wide enough search zone for the re-mapping.

- We also present a heuristic reconfiguration algorithm that could achieve closely to the optimum results, but considerably faster than the optimization solution.

- Different from the previous research studies $[17,60,62,64,69]$ our method does not decrease the network's admittance ratio for new virtual networks. This is because we reconfigure the mapping of the already accepted VNs only for the off-peak period, and they could be reconfigured back to their peak mapping in the case of an unexpected new demand.

- We do not move VMs, so our method does not have the difficulties of [16].

- We define a stress rate for a substrate link. So, we propose a fine-grained local reconfiguration approach that may set a less stressed substrate link into the sleep mode for the off-peak time. Accordingly, we re-map an allocated traffic capacity to a virtual link in a less stressed substrate link if we set the substrate link into the sleep mode. Our solution makes a decision about which allocated traffic capacities of which virtual links, are necessary to be re-mapped. This increases the complexity of the problem in comparison to the classic routing problems in [72-78]. However, it enables the providers to change the level of the reconfiguration by adjusting the stress rate's threshold, and therefore control the possible interruptions. We show the proposed fine-grained local reconfiguration is able to reach the same level of energy-saving as the coarsegrained global reconfiguration (the benchmark), by relaxing the constraint for the stress rate' threshold. This is a novel approach different to any existing research studies.

- As a consequence of this novel approach, our solutions are not limited to a subtopology as the case in [70,76-78], and so it has larger degree of freedom to save energy. The simulation results prove the significant improvement in saving the power by our method, in comparison to the state-of-the-art. 
- We evaluate the proposed solutions by extensive simulations. So, the impact of different factors is studied.

- To the best of our knowledge, there is not such a comprehensive study in the literature that considers simultaneously global/local, coarse-grained/fine-grained reconfiguration of a VNE for splittable/non-splittable traffic, according to two power models.

This chapter is organized as follows: Two physical link power models are reviewed in Section 3.2. The optimization programs are formulated in Section 3.3 and the suggested heuristic is discussed in Section 3.4. The performance of the ILPs as well as the heuristic algorithm are evaluated in Section 3.5. The chapter will conclude in Section 3.6.

\subsection{Power Models}

Various link power models are reviewed in Section 2.3.2. In this chapter, we use two major linear power models to define a physical link's power consumption. We discuss our energy-saving solutions according to Fixed link power model and SemiProportional link power model.

The actual power consumption $\tilde{p}\left(l_{s}^{i, j}\right)$ of a physical link $l_{s}^{i, j}$ could be found by Equation 2.58, according to Fixed link power model. Besides, Equation 2.59 defines $\tilde{p}\left(l_{s}^{i, j}\right)$ of a physical link $l_{s}^{i, j}$, based on Semi-Proportional link power model.

Today's networks are designed based on Fixed link power model, so it is a common model that is widely used [56]. Nevertheless, it is not efficient that an active physical link consumes a constant amount of power, regardless of its traffic load. Therefore, the physical links are expected to get modified, so their power consumption will be more adaptive to their traffic load. The Semi-Proportional link power model brings a traffic-adaptive power model that a physical link's power consumption is changing according to its traffic load. These power models are validated by measurements against the actual physical links in the previous studies, e.g. [81,82]. 


\subsection{Integer Linear Programs}

Towards minimizing the total link energy consumption in a VNE during the off-peak time, the general problem description is the following:

Given:

- A physical substrate network topology

- Allocated virtual networks' topologies

- For each substrate link: Its bandwidth capacity, and every allocated traffic capacity to a virtual link in the substrate link

- Off-peak traffic demands of every virtual network (determined by VNs' customers or the network traffic prediction techniques)

Find:

- The modified off-peak link mapping of the VNE that leads to the minimum link energy consumption of the substrate network, during the off-peak time

Constraints:

- Supporting the off-peak traffic demands

The most optimum result for this problem could be achieved by the coarse-grained global link reconfiguration program. We call this approach, off-peak link energy optimization by global link reconfiguration. We consider the result of this method as the benchmark. Nevertheless, as it is discussed, this method might cause uncontrolled traffic disputations. Therefore, we propose the fine-grained local link reconfiguration program for the defined problem. The latter approach is called off-peak link energy optimization by local link reconfiguration. In this regard, first, we model a VNE mathematically. Then, according to both Fixed and Semi-Proportional link power models, we define ILPs for both of the approaches. Since the traffic type (splittable/nonsplittable) has a major impact on the solution's methodology, we formulate off-peak link energy optimization by local link reconfiguration, for both splittable and nonsplittable traffic. 


\subsubsection{The Network Model}

A substrate network is modelled as a directed graph $G_{s}=\left(V_{s}, E_{s}\right)$ where $V_{s}$ is the set of substrate vertices, and $E_{s}$ is the set of substrate edges. Vertices represent nodes and edges denote links in a network environment. Since the graph is directed, we have a higher level of flexibility in terms of rerouting traffic flows.

Similar to the substrate network model, the $n$th virtual network, from set of all the involved virtual networks $\Phi$, is also modelled as a directed graph $G_{n}=\left(V_{n}, E_{n}\right)$. $V_{n}$ and $E_{n}$ stand for the $n$th virtual network vertices and edges, respectively. $L_{n}=\left|E_{n}\right|$ denotes the total number of virtual links in the $n$th virtual network.

In a VNE embedding procedure, a requested virtual network $G_{n}$ is mapped onto a substrate network $G_{s}: G_{n} \rightarrow G_{s}$. Virtual nodes are embedded onto the qualified substrate nodes. A virtual link could be mapped onto a single substrate link, or multiple substrate links which makes a substrate path. If traffic is splittable, then a requested traffic capacity for a virtual link could be allocated in multiple substrate paths. However, if traffic is non-splittable, then each demanded traffic capacity is allocated only in one path. The allocated virtual links of the $n$th VN are given as a set of ordered allocated virtual node pairs $\left(a_{m}, b_{m}\right), m=1,2, \ldots, L_{n} . l_{n}^{a_{m}, b_{m}}$ represents the $m_{t h}$ virtual link, belonging to the $n$th $\mathrm{VN}$, that connects the virtual node mapped onto the $a_{m}$ th substrate node to the virtual node mapped onto the $b_{m}$ th substrate node. The off-peak traffic demand $r_{n}^{m}$ of each virtual link $l_{n}^{a_{m}, b_{m}}$ is also given. In addition, $\check{C}_{b}^{i, j}\left(l_{n}^{a_{m}, b_{m}}\right)$ represents the allocated traffic capacity to $l_{n}^{a_{m}, b_{m}}$ in $l_{s}^{i, j}$ for the peak time. $\dot{r}_{n}^{i, j}(m)$ denotes the off-peak traffic demand of $\check{C}_{b}^{i, j}\left(l_{n}^{a_{m}, b_{m}}\right)$, and it is known for every allocated traffic capacity in any substrate link. During the the off-peak period, the reserved traffic capacity for $\check{C}_{b}^{i, j}\left(l_{n}^{a_{m}, b_{m}}\right)$ is equal to its off-peak traffic demand $r_{n}^{i, j}(m)$, and the rest of the physical link's bandwidth capacity could be shared. We might aggregate all the allocated traffic capacities in a substrate link. $\check{C}_{b}^{i, j}$ denotes the bundled allocated traffic capacity in a substrate link $l_{s}^{i, j}$, and $r_{b}^{i, j}$ is its associated off-peak traffic demand. Besides, $C_{b}\left(l_{s}^{i, j}\right)$ of each substrate link $l_{s}^{i, j}$ is specified.

For example, Figure 3.1 demonstrates a substrate network and a mapped virtual link $l_{n}^{a_{m}, b_{m}}$ onto the network. Since traffic is splittable in this example, two substrate paths are allocated to the virtual link. Figure 3.1 also shows $\check{C}_{b}^{i, j}\left(l_{n}^{a_{m}, b_{m}}\right)$ and $\check{C}_{b}^{i, j}$ in $l_{s}^{i, j}$. 


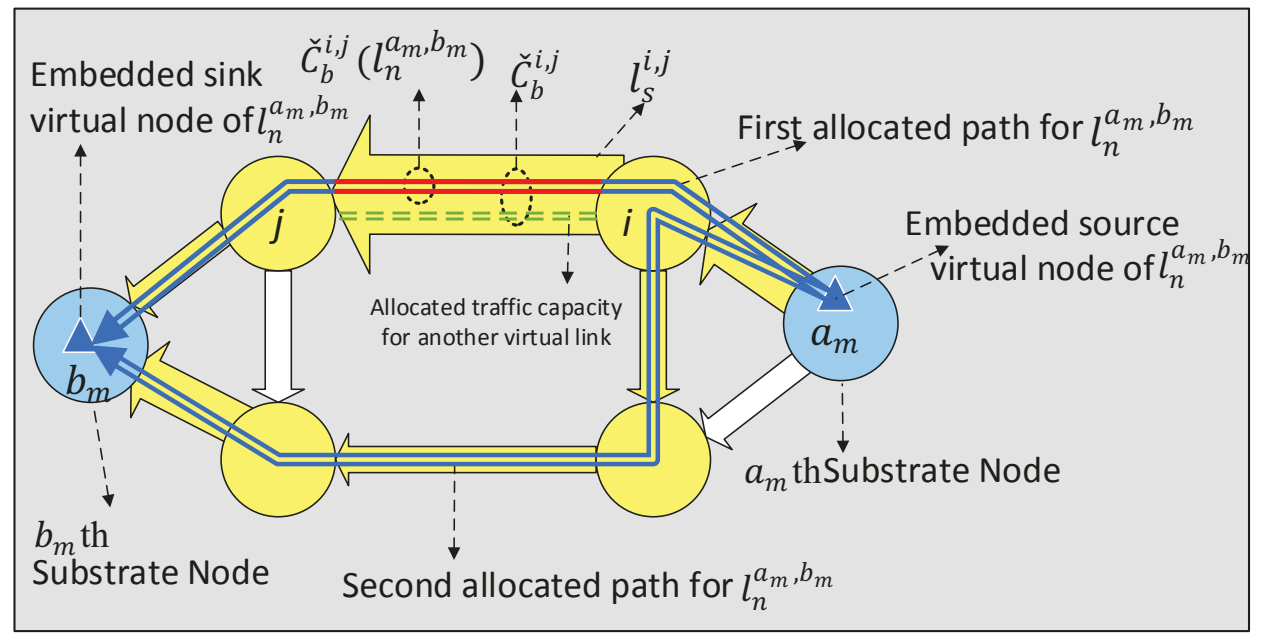

Figure 3.1: Example: An embedded virtual link onto a substrate network

\subsubsection{Programs based on Fixed Link Power Model}

According to Fixed link power model, an active physical link consumes a constant amount of power, regardless of its traffic load. In this chapter, we assume that all the physical links in the substrate network are in the same range of bandwidth capacity. Therefore, active substrate links consume the same amount of power. Accordingly, the energy-saving solution has to put the maximum number of physical links into the sleep mode, in order to minimize the network's link energy consumption. We assume the ideal case, in which a physical link in the sleep mode consumes no power. The following optimization programs in this section are designed according to this methodology.

\section{Off-peak Link Energy Optimization by Global Link Reconfiguration (OL- GLs-F)}

In this section, we intend to re-map every virtual link for the off-peak time, to minimize the number of active substrate links during that period. We re-map every virtual link according to its known off-peak traffic demand. This is a coarse-grained solution, as the granularity is a virtual link. Besides, it is a global optimization, because we remap every virtual link. The traffic is assumed to be splittable in this problem, to give a larger degree of freedom for the re-mapping. This program is expected to deliver the most optimum energy-saving level for our problem. But, it might cause uncontrollable traffic interruptions. We study this program as the benchmark. When this 
problem is formulated according to the Fixed link power model, it is called OL-GLs-F.

This problem could be formulated as a multi-commodity flow problem. In the context of this problem, a virtual link is a commodity. We have $L_{n}$ commodities for the $n$th virtual network. OL-GLs-F is written as an MILP as follows:

\section{Optimization Variables:}

- $\alpha\left(l_{s}^{i, j}\right)$ is an auxiliary binary variable. $\alpha\left(l_{s}^{i, j}\right)$ is 1 when $l_{s}^{i, j}$ is active. Otherwise, $\alpha\left(l_{s}^{i, j}\right)$ is 0 .

- $f_{n}^{i, j}(m)$ is a real-valued variable. $f_{n}^{i, j}(m)$ is the re-allocated traffic capacity to $l_{n}^{a_{m}, b_{m}}$ in $l_{s}^{i, j}$.

Objective Function: The objective function in Equation 3.1 minimizes the number of active substrate links for the off-peak time.

$$
\text { Minimize } \sum_{(i, j) \in E_{s}} \alpha\left(l_{s}^{i, j}\right)
$$

Constraints: The first constraint in Equation 3.2 is the flow conservation constraint that maintains the flow balance on the nodes and re-allocates the off-peak traffic demand of every virtual link.

$$
\begin{aligned}
\sum_{\left\{j \mid(i, j) \in E_{s}\right\}} f_{n}^{i, j}(m)-\sum_{\left\{j \mid(j, i) \in E_{s}\right\}} f_{n}^{j, i}(m)= \begin{cases}r_{n}^{m} & \text { if } i=a_{m} \\
-r_{n}^{m} & \text { if } i=b_{m} \\
0 & \text { otherwise }\end{cases} \\
\forall i \in V_{s}, \forall n \in\left\{n \mid G_{n} \in \Phi\right\}, m=1,2, \ldots, L_{n}
\end{aligned}
$$

The second constraint in Equation 3.3 ensures the total re-allocated traffic capacity $r\left(l_{s}^{i, j}\right)$ in each substrate link $l_{s}^{i, j}$ is less than its physical bandwidth capacity.

$$
r\left(l_{s}^{i, j}\right) \leq C_{b}\left(l_{s}^{i, j}\right), \quad \forall(i, j) \in E_{s}
$$

where: 


$$
r\left(l_{s}^{i, j}\right)=\sum_{\left\{n \mid G_{n} \in \Phi\right\}} \sum_{m=1}^{L_{n}} f_{n}^{i, j}(m)
$$

A constraint in Equation 3.5 is added to make the program linear. Note that $B$ is a large integer number. It must be large enough to be greater than the largest amount of $r\left(l_{s}^{i, j}\right) \cdot r\left(l_{s}^{i, j}\right)$ could be 0 or greater than 0 . First, assume $r\left(l_{s}^{i, j}\right)=0$, so according to the objective function and the constraint in Equation 3.5, $\alpha\left(l_{s}^{i, j}\right)$ will be 0. Second, assume $r\left(l_{s}^{i, j}\right)>0$. In this case, $\alpha\left(l_{s}^{i, j}\right)$ must be 1 to satisfy the constraint.

$$
r\left(l_{s}^{i, j}\right) \leq B \alpha\left(l_{s}^{i, j}\right), \quad \forall(i, j) \in E_{s}
$$

In addition, the variables must hold the following bounds:

$$
\begin{gathered}
f_{n}^{i, j}(m) \geq 0, \quad \forall(i, j) \in E_{s}, \forall n \in\left\{n \mid G_{n} \in \Phi\right\}, m=1,2, \ldots, L_{n} \\
\alpha\left(l_{s}^{i, j}\right) \in\{0,1\}, \quad \forall(i, j) \in E_{s}
\end{gathered}
$$

The formulated MILP for OL-GLs-F is a type of VNE embedding problems. An MILP is $\mathcal{N} \mathcal{P}$-hard in general, because an ILP is $\mathcal{N} \mathcal{P}$-hard. Besides, a VNE embedding process is $\mathcal{N} \mathcal{P}$-hard [15]. In consequence, the defined MILP for off-peak link energy optimization by global link reconfiguration is $\mathcal{N} \mathcal{P}$-hard, and therefore the optimization solution is not scalable to the large network sizes.

\section{Off-peak Link Energy Optimization by Local Link Reconfiguration}

The previous reconfiguration approach might cause uncontrolled traffic interruptions, as discussed. Therefore, we propose a second methodology. First, we define a stress rate for a substrate link. Then, we develop solutions that may set a less stressed substrate link into the sleep mode for the off-peak time. We re-map an allocated traffic capacity to a virtual link in a less stressed substrate link if we set the substrate link into the sleep mode. Different from the previous approach, this is a fine-grained solution as the granularity is an allocated traffic capacity to a virtual link, in a substrate link. Besides, this is a local optimization, because it does not re-map every allocated traffic capacity in any physical link. This method enables the providers to 
change the level of the reconfiguration by adjusting the stress rate's threshold, and therefore control the possible traffic interruptions.

The stress rate $\tilde{s}\left(l_{s}^{i, j}\right)$ of a substrate link $l_{s}^{i, j}$ denotes the intensity of involved VNs and the total off-peak traffic demand in the link. A VN is involved in a substrate link $l_{s}^{i, j}$, if at least one of its embedded virtual links passes through $l_{s}^{i, j}$. Assume $\eta\left(l_{s}^{i, j}\right)$ as the number of VNs involved in a substrate link $l_{s}^{i, j}$, Equation 3.8 defines $\tilde{s}\left(l_{s}^{i, j}\right)$.

$$
\tilde{s}\left(l_{s}^{i, j}\right)=\frac{\eta\left(l_{s}^{i, j}\right)}{|\Phi|} \times \frac{\sum_{\left\{n \mid G_{n} \in \Phi\right\}} \sum_{m=1}^{L_{n}} \dot{r}_{n}^{i, j}(m)}{C_{b}\left(l_{s}^{i, j}\right)}
$$

$\tilde{s}\left(l_{s}^{i, j}\right)$ considers two parameters. The first parameter $\left(\frac{\eta\left(l_{s}^{i, j}\right)}{|\Phi|}\right)$ is the fraction of the number of involved VNs in a substrate link, over the total number of active VNs. This parameter denotes the intensity of the involved VNs in an $l_{s}^{i, j}$. This is an important factor. If a substrate link is highly intense in regard to the involved VNs, then traffic of the large number of involved VNs passes through the link. Therefore, sleeping such a substrate link might affect normal operations in a large number of VNs. The second parameter (all the terms except the first parameter) considers the off-peak traffic demand by finding the fraction of the total off-peak traffic passes a substrate link, over the bandwidth capacity of the link. This is essential, since sleeping a substrate link with a high traffic utilization might cause large traffic interruptions. A higher link stress rate means a larger number of VNs is involved in the link, or the link is more utilized for the off-peak time. In this regard, we do not re-map the allocated traffic capacities in a substrate link with $\tilde{s}\left(l_{s}^{i, j}\right) \geq \mathcal{T}$, in order to control the traffic disruptions due to the reconfiguration. $\mathcal{T}$ is a stress rate's threshold, and it is a real number between 0 and 1 . Providers could adjust $\mathcal{T}$. Decreasing the value of $\mathcal{T}$ degrades the amount of energy the programs could save, but also reduces the traffic interruptions due to the reconfiguration. This is because a smaller number of physical links are considered for energy-saving. The impact of setting different values of $\mathcal{T}$ on energy-saving ability of the solutions is discussed in Section 3.5.

The traffic might be splittable or non-splittable. In the splittable case, the traffic demand of a virtual link could be carried by one or multiple paths in a substrate network. However, if the traffic is non-splittable, then a virtual link's traffic demand may be required to follow the same path through the network, rather than be divided among multiple paths. It is expected to save a higher amount of energy when traffic is splittable, because we are more flexible in terms of finding alternative paths 
during the reconfiguration. This is a major restriction that has an important impact on the solution's methodology. In this regard, we formulate the off-peak link energy optimization by local link reconfiguration, for both splittable and non-splittable traffic.

Splittable Traffic (OL-LLs-F) Because the traffic is assumed to be splittable, it is the same to aggregate all the allocated traffic capacities in a physical link and then re-allocate the bundled traffic capacity onto multiple paths, or re-allocate every single allocated traffic capacity in the link onto multiple paths. In order to simplify the program we re-allocate the bundled traffic capacities according to their off-peak traffic demand. In this problem, we re-map the bundled allocated traffic capacity in a less stressed substrate link if we set the substrate link into the sleep mode. The off-peak link energy optimization by local link reconfiguration problem for splittable traffic, that is defined according to Fixed link power model, is called OL-LLs-F.

The defined reconfiguration problem could be formulated as a multi-commodity flow problem. In the context of this problem, the bundled allocated traffic capacity $\check{C}_{b}^{i, j}$ in a substate link $l_{s}^{i, j}$ with $\tilde{s}\left(l_{s}^{i, j}\right)<\mathcal{T}$, is a commodity. Each commodity $\check{C}_{b}^{i, j}$ is associated with a known off-peak traffic demand $r_{b}^{i, j}$. OL-LLs-F is formulated as an MILP as follows:

Optimization Variables:

- $\alpha\left(l_{s}^{i, j}\right)$ is an auxiliary binary variable. $\alpha\left(l_{s}^{i, j}\right)$ is 1 when a substrate link $l_{s}^{i, j}$ is active, otherwise $\alpha\left(l_{s}^{i, j}\right)$ is 0 .

- $f^{x, y}\left(\check{C}_{b}^{i, j}\right)$ is a real-valued variable. It is the re-allocated traffic capacity to $\check{C}_{b}^{i, j}$ in $l_{s}^{x, y} . l_{s}^{x, y}$ is a substrate link that connects the $x$ th substrate node to the $y$ th substrate node. Similarly, $f^{i, j}\left(\check{C}_{b}^{x, y}\right)$ is a real-valued variable.

- $\beta\left(\check{C}_{b}^{i, j}\right)$ is a binary variable. It shows $\check{C}_{b}^{i, j}$ status, after the reconfiguration. It is 0 in the case $\check{C}_{b}^{i, j}$ is removed, after the reconfiguration. Otherwise, $\beta\left(\check{C}_{b}^{i, j}\right)$ is 1 . Objective Function: The same objective as Equation 3.1.

Constraints: The constraints in Equations 3.3, 3.5, and the followings: The constraint in Equation 3.9 is the flow conservation constraint that re-allocates the off-peak traffic demand of a removed commodity. The program does not re-allocate the off-peak traffic 
demand of every bundled allocated traffic capacity. It re-allocates the off-peak traffic demand of a commodity if the commodity is removed. If the program decides to remove a bundled allocated traffic capacity $\check{C}_{b}^{i, j}$, then $\check{C}_{b}^{i, j}$ status changes, and so $1-\beta\left(\check{C}_{b}^{i, j}\right)$ is equal to 1. Therefore, the constraint in Equation 3.9 requires re-allocating one or multiple alternative paths from the $i$ th substrate node to the $j$ th substate node, which support its off-peak traffic demand $\dot{r}_{b}^{i, j}$. Nevertheless, if $\check{C}_{b}^{i, j}$ is not removed during the reconfiguration process, then $1-\beta\left(\check{C}_{b}^{i, j}\right)$ is equal to 0 , and therefore $\check{C}_{b}^{i, j}$ is not re-mapped.

$$
\sum_{\left\{y \mid(x, y) \in E_{s}\right\}} f^{x, y}\left(\check{C}_{b}^{i, j}\right)-\sum_{\left\{y \mid(y, x) \in E_{s}\right\}} f^{y, x}\left(\check{C}_{b}^{i, j}\right)=\left\{\begin{array}{rr}
\left(1-\beta\left(\check{C}_{b}^{i, j}\right)\right) \dot{r}_{b}^{i, j} & \text { if } \quad x=i \\
\left(\beta\left(\check{C}_{b}^{i, j}\right)-1\right) \dot{r}_{b}^{i, j} & \text { if } \quad x=j \\
0 & \text { otherwise } \\
& \forall x \in V_{s}, \forall(i, j) \in E_{s}
\end{array}\right.
$$

In this problem, different from OL-GLs-F, there might be two types of allocated traffic capacities in a substrate link, during the off-peak time. The first type is an un-reconfigured bundled allocated traffic capacity. This means $\check{C}_{b}^{i, j}$ was allocated and it is not removed in the reconfiguration process, therefore $\beta\left(\check{C}_{b}^{i, j}\right)=1$. In this case, $\beta\left(\check{C}_{b}^{i, j}\right) \dot{r}_{b}^{i, j}$ is equal to $\dot{r}_{b}^{i, j}$, that is its reserved amount of traffic capacity for the off-peak period. If the program re-allocates $\check{C}_{b}^{i, j}$, then $\beta\left(\check{C}_{b}^{i, j}\right)=0$. So, its original allocated traffic capacity is no longer reserved. The second type is a re-allocated bundled traffic capacity of other links (like $\left.l_{s}^{x, y}\right)$, in a substrate link $l_{s}^{i, j} \cdot f^{i, j}\left(\check{C}_{b}^{x, y}\right)$ is the re-allocated traffic capacity to $\check{C}_{b}^{x, y}$ in $l_{s}^{i, j}$, for the off-peak period. Equation 3.10 calculates the total allocated traffic capacity $r\left(l_{s}^{i, j}\right)$ in $l_{s}^{i, j}$, for this problem.

$$
r\left(l_{s}^{i, j}\right)=\beta\left(\check{C}_{b}^{i, j}\right) r_{b}^{i, j}+\sum_{(x, y) \in E_{s}} f^{i, j}\left(\check{C}_{b}^{x, y}\right)
$$

Moreover, the constraint in Equation 3.11 avoids re-mapping a bundled allocated traffic capacity in a substrate link with $\tilde{s}\left(l_{s}^{i, j}\right) \geq \mathcal{T}$, in order to decrease the traffic interruptions. This constraint enables the providers to control the level of the reconfiguration, and therefore control the possible interruptions. 


$$
\beta\left(\check{C}_{b}^{i, j}\right)=1, \quad \forall(i, j) \in\left\{(i, j) \mid(i, j) \in E_{s}, \tilde{s}\left(l_{s}^{i, j}\right) \geq \mathcal{T}\right\}
$$

Furthermore, the variables must hold the bound in Equation 3.7, and the followings:

$$
\begin{gathered}
f^{x, y}\left(\check{C}_{b}^{i, j}\right) \geq 0, \quad \forall(x, y) \in E_{s}, \forall(i, j) \in E_{s} \\
\beta\left(\check{C}_{b}^{i, j}\right) \in\{0,1\}, \quad \forall(i, j) \in E_{s}
\end{gathered}
$$

Non-Splittable Traffic (OL-LLns-F) Since the traffic is non-splittable, it is not possible to re-allocate an allocated traffic capacity to a virtual link in a substrate link, onto multiple substrate paths. Consequently, aggregating all the allocated traffic capacities in a substrate link and then re-allocating the bundled traffic capacity, is not an efficient approach. This is because there would be a smaller number of alternative paths that could support the bundled off-peak traffic demand. In this problem, we remap an allocated traffic capacity to a virtual link in a less stressed substrate link if we set the substrate link into the sleep mode. We also might re-map an allocated traffic capacity to a virtual link in a less stressed substrate link if re-mapping the traffic capacity provides enough bandwidth capacity in the substrate link for re-mapping of another traffic capacity, which leads to the minimum total link energy consumption. The off-peak link energy optimization by local link reconfiguration for non-splittable traffic, that is formulated according to the Fixed link power model, is called OL-LLnsF.

This problem could be formulated as a BILP in the category of multi-commodity flow problems. Different from splittable form, an allocated traffic capacity $\check{C}_{b}^{i, j}\left(l_{n}^{a_{m}, b_{m}}\right)$ to a virtual link $l_{n}^{a_{m}, b_{m}}$ in a substrate link $l_{s}^{i, j}$ with $\tilde{s}\left(l_{s}^{i, j}\right)<\mathcal{T}$, is a commodity. OL-LLns-F is formulated as a BILP as follows:

\section{Optimization Variables:}

- $\alpha\left(l_{s}^{i, j}\right)$ is an auxiliary binary variable. $\alpha\left(l_{s}^{i, j}\right)$ is 1 when a physical link $l_{s}^{i, j}$ is active, otherwise $\alpha\left(l_{s}^{i, j}\right)$ is 0 . 
- $z^{x, y}\left(\check{C}_{b}^{i, j}\left(l_{n}^{a_{m}, b_{m}}\right)\right)$ is a binary variable. If the re-allocated path for a commodity $\check{C}_{b}^{i, j}\left(l_{n}^{a_{m}, b_{m}}\right)$ passes through $l_{s}^{x, y}$, then $z^{x, y}\left(\check{C}_{b}^{i, j}\left(l_{n}^{a_{m}, b_{m}}\right)\right)=1$. Otherwise, $z^{x, y}\left(\check{C}_{b}^{i, j}\left(l_{n}^{a_{m}, b_{m}}\right)\right)=0$. Similarly, $z^{i, j}\left(\check{C}_{b}^{x, y}\left(l_{n}^{a_{m}, b_{m}}\right)\right)$ is a binary variable.

- $\beta\left(\check{C}_{b}^{i, j}\left(l_{n}^{a_{m}, b_{m}}\right)\right)$ is a binary variable. It shows $\check{C}_{b}^{i, j}\left(l_{n}^{a_{m}, b_{m}}\right)$ status, after the reconfiguration. It is 0 in the case $\check{C}_{b}^{i, j}\left(l_{n}^{a_{m}, b_{m}}\right)$ is removed, after the reconfiguration. Otherwise, $\check{C}_{b}^{i, j}\left(l_{n}^{a_{m}, b_{m}}\right)$ is 1 .

Objective Function: The same objective as Equation 3.1.

Constraints: The constraints in Equations 3.3, 3.5, and the followings: If the program decides to remove an allocated traffic capacity $\check{C}_{b}^{i, j}\left(l_{n}^{a_{m}, b_{m}}\right)$, then $\beta\left(\check{C}_{b}^{i, j}\left(l_{n}^{a_{m}, b_{m}}\right)\right)$ will be equal to 0. Therefore, Equation 3.14 needs to route a single unit of data from the $i$ th substrate node to the $j$ th substrate node. Because the variable $z^{x, y}\left(\check{C}_{b}^{i, j}\left(l_{n}^{a_{m}, b_{m}}\right)\right)$ is binary, the unit of data could not be splitted. Besides, the constraint in Equation 3.15 limits the program routing, so the maximum number of incoming and outgoing flows of every commodity, in any node, is two. This maintains a single loopless path. Thus, the driven route will be used as a replaced path for $\check{C}_{b}^{i, j}\left(l_{n}^{a_{m}, b_{m}}\right)$. If an allocated traffic capacity $\check{C}_{b}^{i, j}\left(l_{n}^{a_{m}, b_{m}}\right)$ is not removed $\left(\beta\left(\check{C}_{b}^{i, j}\left(l_{n}^{a_{m}, b_{m}}\right)\right)=1\right)$, then it will not be re-allocated.

$$
\begin{aligned}
& \sum_{\left\{y \mid(x, y) \in E_{s}\right\}} z^{x, y}\left(\check{C}_{b}^{i, j}\left(l_{n}^{a_{m}, b_{m}}\right)\right)-\sum_{\left\{y \mid(y, x) \in E_{s}\right\}} z^{y, x}\left(\check{C}_{b}^{i, j}\left(l_{n}^{a_{m}, b_{m}}\right)\right) \\
& =\left\{\begin{array}{l}
1-\beta\left(\check{C}_{b}^{i, j}\left(l_{n}^{a_{m}, b_{m}}\right)\right) \text { if } x=i \\
\beta\left(\check{C}_{b}^{i, j}\left(l_{n}^{a_{m}, b_{m}}\right)\right)-1 \quad \text { if } \quad x=j, \\
0
\end{array} \quad\right. \text { otherwise } \\
& \forall x \in V_{s}, \forall n \in\left\{n \mid G_{n} \in \Phi\right\}, m=1,2, \ldots, L_{n}, \forall(i, j) \in E_{s}
\end{aligned}
$$


The total allocated traffic capacity $r\left(l_{s}^{i, j}\right)$ in a physical link $l_{s}^{i, j}$ during the off-peak period, is the summation of the total un-reconfigured allocated traffic capacities $\left(\beta\left(\check{C}_{b}^{i, j}\left(l_{n}^{a_{m}, b_{m}}\right)=1\right)\right)$ in $l_{s}^{i, j}$ as well as the re-allocated traffic capacities of other links (like $\left.l_{s}^{x, y}\right)$ in $l_{s}^{i, j} \cdot r\left(l_{s}^{i, j}\right)$ is calculated in Equation 3.16. $z^{i, j}\left(\check{C}_{b}^{x, y}\left(l_{n}^{a_{m}, b_{m}}\right)\right) r_{n}^{x, y}(m)$ is the re-allocated traffic capacity to $\check{C}_{b}^{x, y}\left(l_{n}^{a_{m}, b_{m}}\right)$ in $l_{s}^{i, j}$.

$$
\begin{aligned}
r\left(l_{s}^{i, j}\right)=\sum_{\left\{n \mid G_{n} \in \Phi\right\}} \sum_{m=1}^{L_{n}}\left\{\beta\left(\check{C}_{b}^{i, j}\left(l_{n}^{a_{m}, b_{m}}\right)\right) \dot{r}_{n}^{i, j}(m)\right\} \\
\\
\quad+\sum_{(x, y) \in E_{s}} \sum_{\left\{n \mid G_{n} \in \Phi\right\}} \sum_{m=1}^{L_{n}}\left\{z^{i, j}\left(\check{C}_{b}^{x, y}\left(l_{n}^{a_{m}, b_{m}}\right)\right) \dot{r}_{n}^{x, y}(m)\right\}
\end{aligned}
$$

The constraint in Equation 3.17 prevents the program from re-mapping an allocated traffic capacity in a physical link $l_{s}^{i, j}$ with $\tilde{s}\left(l_{s}^{i, j}\right) \geq \mathcal{T}$.

$$
\beta\left(\check{C}_{b}^{i, j}\left(l_{n}^{a_{m}, b_{m}}\right)\right)=1, \quad \forall(i, j) \in\left\{(i, j) \mid(i, j) \in E_{s}, \tilde{s}\left(l_{s}^{i, j}\right) \geq \mathcal{T}\right\}, \forall n \in\left\{n \mid G_{n} \in \Phi\right\},
$$

In addition, the variables must hold the bound in Equation 3.7, and the followings:

$$
\begin{array}{r}
z^{x, y}\left(\check{C}_{b}^{i, j}\left(l_{n}^{a_{m}, b_{m}}\right)\right) \in\{0,1\}, \quad \forall(x, y) \in E_{s}, \forall n \in\left\{n \mid G_{n} \in \Phi\right\}, m=1,2, \ldots, L_{n} \\
\forall(i, j) \in E_{s}
\end{array}
$$

$$
\beta\left(\check{C}_{b}^{i, j}\left(l_{n}^{a_{m}, b_{m}}\right)\right) \in\{0,1\}, \quad \forall(i, j) \in E_{s}, \forall n \in\left\{n \mid G_{n} \in \Phi\right\}, m=1,2, \ldots, L_{n}
$$

The formulated integer linear programs for off-peak link energy optimization by local link reconfiguration, either for splittable traffic (OL-LLs-F), or non-splittable traffic (OL-LLns-F), could be reduced to the problem discussed in [83] that is a simple two-commodity integer flow problem. It is proven in [83] that this simple two-commodity integer flow problem is $\mathcal{N} \mathcal{P}$-hard. Hence, the formulated programs are $\mathcal{N} \mathcal{P}$-hard. 


\subsubsection{Programs based on Semi-Proportional Link Power Model}

The previous section developed energy-saving programs for a VNE's links, conforming to Fixed link power model. As it is discussed in Section 3.2, Semi-Proportional link power model defines a traffic-adaptive power model for a physical link. Based on this link power model, a large portion of consumed power by a physical link is for keeping the link operational. Nonetheless, different from Fixed link power model, the traffic load in a link also changes its power consumption. Hence, every physical link in a substrate network does not consume the same amount of power. In this regard, it is possible reduce the link energy consumption by either setting the link into the sleep mode, or by rerouting its traffic load to other physical links with higher bandwidth capacity. However, we could save a larger amount of power by sleeping the link, in comparison to rerouting its traffic load.

The objective functions in the previous programs are required to be modified, so they optimize the energy based on Semi-Proportional link power model. In this regard, the objective function in Equation 3.1 needs to be replaced by Equation 3.20.

$$
\text { Minimize } \sum_{(i, j) \in E_{s}}\left\{\alpha\left(l_{s}^{i, j}\right) \tilde{p}^{b}\left(l_{s}^{i, j}\right)+\frac{r\left(l_{s}^{i, j}\right)}{C_{b}\left(l_{s}^{i, j}\right)}\left(\tilde{p}^{m}\left(l_{s}^{i, j}\right)-\tilde{p}^{b}\left(l_{s}^{i, j}\right)\right)\right\}
$$

By modifying the objective function and keeping the same constraints and bounds, the programs reconfigure the mapping of allocated VNs, according to SemiProportional link power model defined in Equation 2.59.

Note that the off-peak link energy optimization by global link reconfiguration problem, which is formulated according to Semi-Proportional link power model, is called OL-GLs-SP. Besides, the off-peak link energy optimization by local link reconfiguration problem that is defined according to Semi-Proportional link power model is called OL-LLs-SP in the case of splittable traffic, and OL-LLns-SP in the case of non-splittable traffic. 


\subsection{The Heuristic Algorithm}

The discussed BILP for OL-LLns-F in Section 3.3.2 is $\mathcal{N} \mathcal{P}$-hard, and therefore the optimization solution is not scalable to large network sizes, due to its long executing time. In this section, we propose a scalable heuristic algorithm for OL-LLns-F. The pseudo code of the proposed heuristic algorithm is shown in Algorithm 1.

We know from Semi-Proportional power models that a very large part of power consumption of a physical link is for its base power consumption rather than the traffic related power consumption. Therefore, targeting only the base power consumption of a link still provides an effective energy-saving method. Hence, to simplify the heuristic and decrease its time complexity, it is defined based on Fixed power model. This means the heuristic does not save energy by rerouting the traffic.

The algorithm checks the possibility of setting a VNE's physical links into the sleep mode during the off-peak time. This process must be performed precisely in order to guarantee that the network supports the off-peak traffic demands of all the involved VNs. In this regard, the algorithm first calculates some metrics and then sorts the physical links in order to check the link removal possibility. Afterwards, it attempts to find an alternative path for the off-peak traffic demand of every allocated traffic capacity to a virtual link in a removed physical link. This process is performed in multiple phases.

During the off-peak period, the available bandwidth capacity in an $l_{s}^{i, j}$ is represented by $\breve{C}_{b}\left(l_{s}^{i, j}\right) . \breve{C}_{b}\left(l_{s}^{i, j}\right)$ is equal to its physical capacity subtracted by the total reserved off-peak traffic capacities for virtual links in $l_{s}^{i, j}$. Equation 3.21 defines $\breve{C}_{b}\left(l_{s}^{i, j}\right)$. Besides, $G_{s}^{T}$ is the off-peak substrate topology. At first, $G_{s}^{T}$ is the same as the substrate network topology.

$$
\breve{C}_{b}\left(l_{s}^{i, j}\right)=C_{b}\left(l_{s}^{i, j}\right)-\sum_{\left\{n \mid G_{n} \in \Phi\right\}} \sum_{m=1}^{L_{n}} r_{n}^{i, j}(m)
$$

Because the substrate links with a higher stress rate are more essential in regard to the traffic demands and the possible interruptions, the algorithm starts setting substrate links into the sleep mode from the link that has the lowest stress rate. It sorts the substrate links with $\tilde{s}\left(l_{s}^{i, j}\right)<\mathcal{T}$ in ascending order based on $\tilde{s}$. The list is 


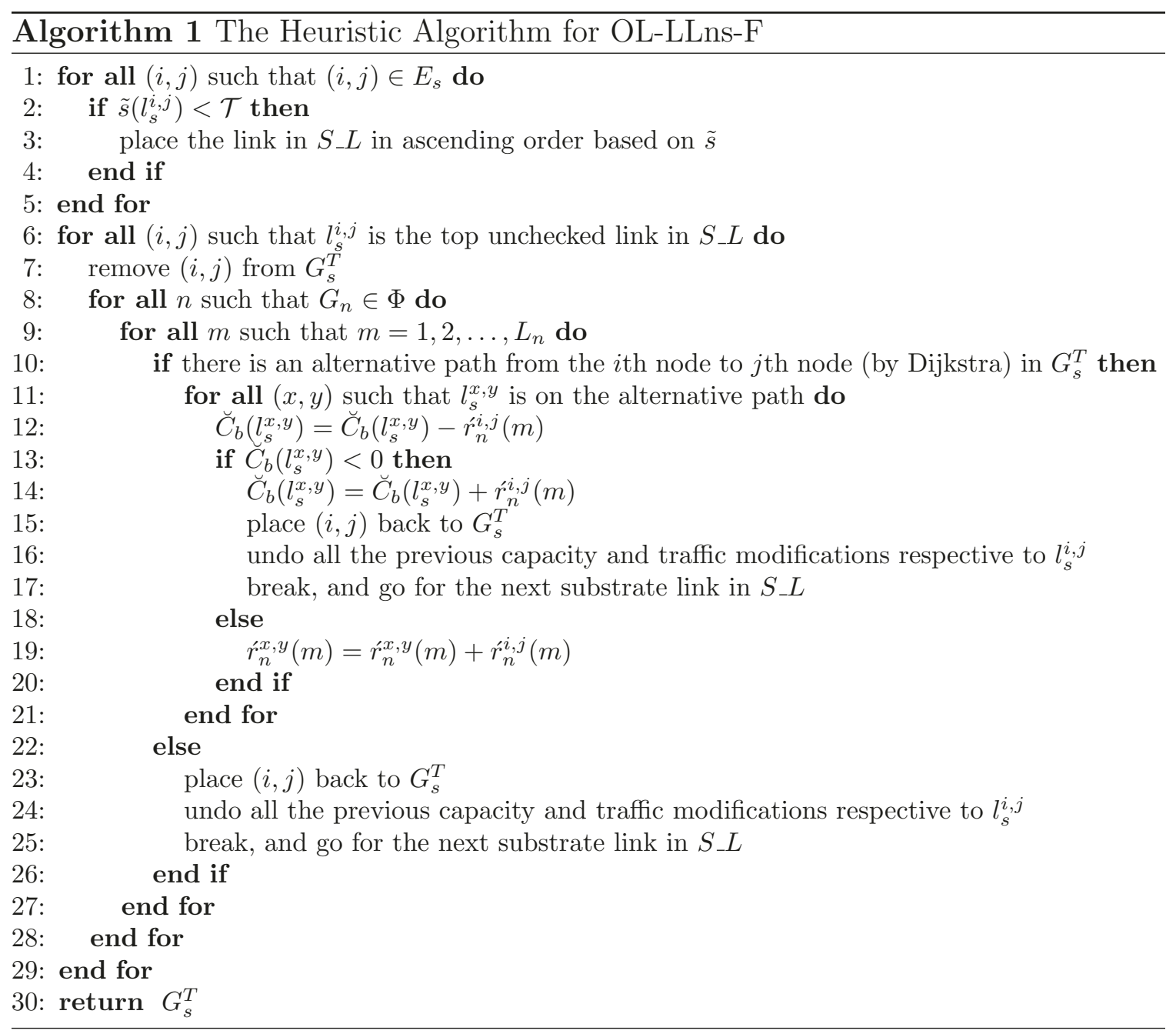

represented by $S_{-} L$.

In the next phase, the algorithm removes the physical links that are capable to be set into the sleep mode, from $G_{s}^{T}$, and at the end it returns $G_{s}^{T}$ as the energy-efficient off-peak substrate topology. This phase also ensures the rearranged network accommodates the off-peak traffic demands. In this regards, there must be a single replaced path for each removed traffic capacity that supports its off-peak traffic demand. The algorithm attempts to find such an alternative path for every allocated traffic capacity to a virtual link in every physical link with $\tilde{s}\left(l_{s}^{i, j}\right)<\mathcal{T}$. The algorithm uses Dijkstra algorithm as the preferred routing algorithm to find the shortest alternative path, while every active physical link cost is assumed as 1. It is needed to check the eligibility of every substrate link on the path in regard to the available off-peak bandwidth capacity. Three conditions might happen while the algorithm searches for 
such a replaced path:

- There is such an alternative path in $G_{s}^{T}$. So, the algorithm updates the respective $\breve{C}_{b}$, and allocates the respective traffic capacity in all the substrate links over the path.

- There is an alternative path in $G_{s}^{T}$, but one or some of the substrate links over the path do not support the off-peak traffic demand. Therefore, the algorithm places the respective physical link back to $G_{s}^{T}$, cancels all the previous capacity and traffic modifications, and aborts the checking process for the rest of the allocated traffic capacities in this physical link.

- There is no alternative path in $G_{s}^{T}$. Hence, the algorithm places back the respective physical link to $G_{s}^{T}$, cancels all the previous capacity and traffic modifications, and aborts the checking process for the rest of the allocated traffic capacities in this physical link.

After the checking process for all of the removed physical links, $G_{s}^{T}$ is returned as the energy-efficient off-peak substrate topology.

It is expected the suggested heuristic for OL-LLns-F is considerably simpler and faster than the BILP. The largest loop, starts in line 6 and ends in line 29, determines the complexity of the proposed heuristic. This loop runs for every physical link, so its complexity is $\mathcal{O}\left(\left|E_{s}\right|\right)$. The first sub-loop starts in line 8 runs for every $\mathrm{VN}$, and therefore its complexity is $\mathcal{O}(|\Phi|)$. The second sub-loop starts in line 9 runs for every virtual link in the respective virtual network. Considering the worst case, the complexity of this sub-loop is $\mathcal{O}\left(\left|E_{v}^{m}\right|\right)$, where $E_{v}^{m}$ is the set of edges of the involved virtual network with the largest number of virtual links. The heuristic calls Dijkstra algorithm in line 10. The complexity of Dijkstra algorithm in the worst case is $\left.\mathcal{O}\left(\left|E_{s}\right|+\left|V_{s}\right| \log V_{s} \mid\right)\right)$. The third sub-loop starting in line 11 checks the capability of every substrate link on the found path. So, its complexity is $\mathcal{O}\left(\left|E_{s}\right|\right)$. In the worstcase scenario, the heuristic might need to check all the physical links again, in order to undo the capacity and traffic modifications for each re-allocated traffic capacity. So, the complexity of the undoing function is $\mathcal{O}\left(\left|E_{s}\right||\Phi|\left|E_{v}^{m}\right|\right)$. Hence, the complexity of the proposed heuristic is $\mathcal{O}\left(\left|E_{s}\right|^{3}|\Phi|^{2}\left|E_{v}^{m}\right|^{2}\left(\left|E_{s}\right|+\left|V_{s}\right| \log \left|V_{s}\right|\right)\right)$. Consequently, the proposed heuristic algorithm is considerably simpler and it could be solved in a polynomial time. 


\subsection{Evaluation}

The proposed energy-saving solutions are supposed to reduce the total link energy consumption in a VNE during the off-peak time. However, they need to guarantee the off-peak traffic requirements. In order to evaluate their effectiveness, several random VNE setups have been evaluated.

Recently, Waxman algorithm [84] is widely used by the researchers to generate random virtual/substrate topologies for VNEs [17,69,85-87]. Therefore, in this chapter, substrate and virtual networks' topologies are generated by Waxman algorithm. Waxman generates random network topologies based on two parameters, $\tilde{\lambda}$ and $\tilde{\mu}$. As $\tilde{\lambda}$ grows the probability of having an edge between any pair of nodes in the topology is increased. As $\tilde{\mu}$ grows there is a larger ratio of long edges to short edges. In this chapter, we choose the Waxman parameters, for both substrate and virtual networks' topologies, as $\tilde{\lambda}=\tilde{\mu}=0.5$, in the area size of $100 \times 100$. After creating random substrate and virtual networks' topologies, the substrate links' capacities and virtual links' peak demands are generated randomly with a uniform distribution. The bandwidth capacity of a physical link is a random amount between $100 \mathrm{Mbps}$ and $200 \mathrm{Mbps}$, but a virtual link's bandwidth demand is generated randomly between 40Mbps and $80 \mathrm{Mbps}$. Both randomly generated substrate and virtual networks are symmetric, so if there is a link from the $i$ th node to the $j$ th node with a specific amount of bandwidth capacity, then there is also a link from the $j$ th node to the $i$ th node with the same amount of the bandwidth capacity. In the next step, the created virtual nodes are mapped to the substrate nodes randomly with the uniform distribution. Afterwards, every generated virtual link's peak bandwidth demand is allocated on a substrate path through a state-of-the-art heuristic algorithm.

For example, Figure 3.2a shows a randomly generated virtual network. Besides, Figure $3.2 \mathrm{~b}$ demonstrates a randomly generated substrate network that includes allocated nodes and links of the generated virtual network in Figure 3.2a.

As it is discussed, the formulated ILPs are $\mathcal{N} \mathcal{P}$-hard, so they are not scalable to large network sizes. Therefore, we assess the capability of the defined ILPs on small random simulation setups, similar to the other related works in $[17,56,69,70]$. The scale of a small random simulation setup is comparable to GÉANT network that is a real universal network. GÉANT has 22 nodes and 36 bidirectional links. The ILPs are solved by MOSEK solver [88]. Nonetheless, the theoretical complexity analysis reveals that the proposed heuristic algorithm is considerably simpler, and therefore it 
is scalable to large network sizes. Hence, the performance of the suggested heuristic is examined on large random simulation setups.

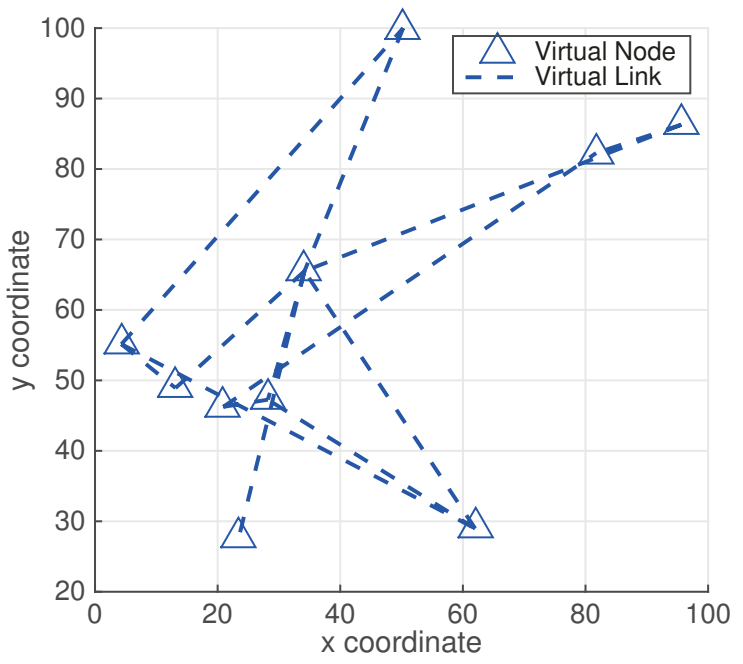

(a) A Random VN

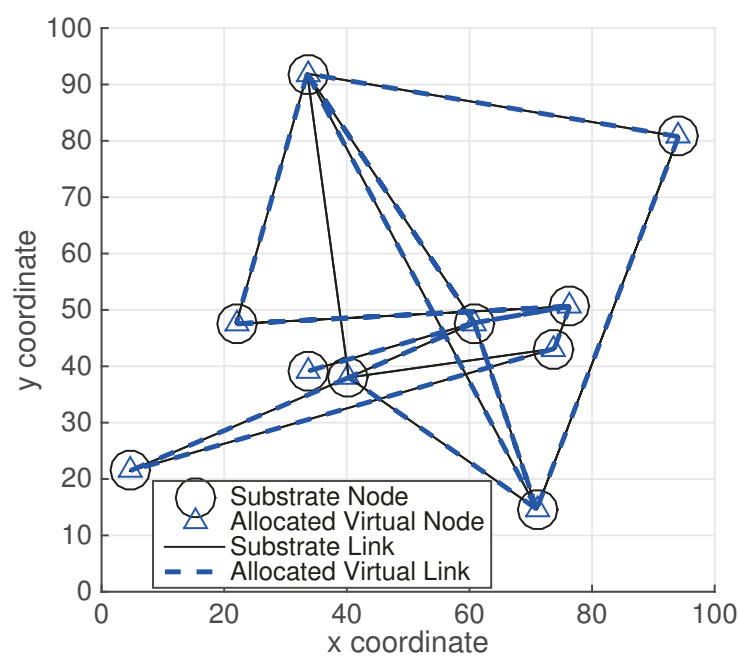

(b) A Random Substrate Network

Figure 3.2: Example: Generated random topologies

Every small random simulation setup contains 10 randomly generated VNEs. Each VNE in a small random simulation setup has 2 random virtual networks that are allocated on a single random substrate network, while every substrate and virtual network has 10 nodes. The average number of physical links in the small random simulation setups is 30. Furthermore, every large random simulation setup includes 10 randomly generated VNEs. All the VNEs in a large random simulation setup have at least 2 random virtual networks that are mapped on a single random substrate network, while the substrate network has 50 physical nodes and each virtual network has 20 virtual nodes. The average number of physical links in the large random simulation setups is 590 . We assume $\mathcal{T}=0.6$, unless otherwise stated. The average results including confidence intervals with the confidence level of $90 \%$ are calculated for each setup. Assuming $\bar{x}$ as an average result, a confidence interval is calculated by Equation 3.22, where $\sigma$ is the standard deviation.

$$
\bar{x} \pm 1.645 \frac{\sigma}{\sqrt{10}}
$$




\subsubsection{ILPs}

First, we solved the formulated MILPs for OL-GLs-F and OL-LLs-F on a small random simulation setup, while traffic is assumed to be splittable. Both have been solved for different amounts of the off-peak traffic ratio. An off-peak traffic ratio is a fraction of the network's off-peak traffic rate by its peak traffic rate. The average number of physical links in the sleep mode during the off-peak period has been probed and shown in Figure 3.3. The results illustrate that both of OL-GLs-F and OL-LLs-F are able to set a notable number of physical links into the sleep mode during this time. Besides, the number of physical links in the sleep mode is decreasing by increasing the off-peak traffic ratio. This is because increasing the off-peak traffic ratio increases the amount of traffic programs need to re-allocate, so they are more limited in terms of finding alternative paths.

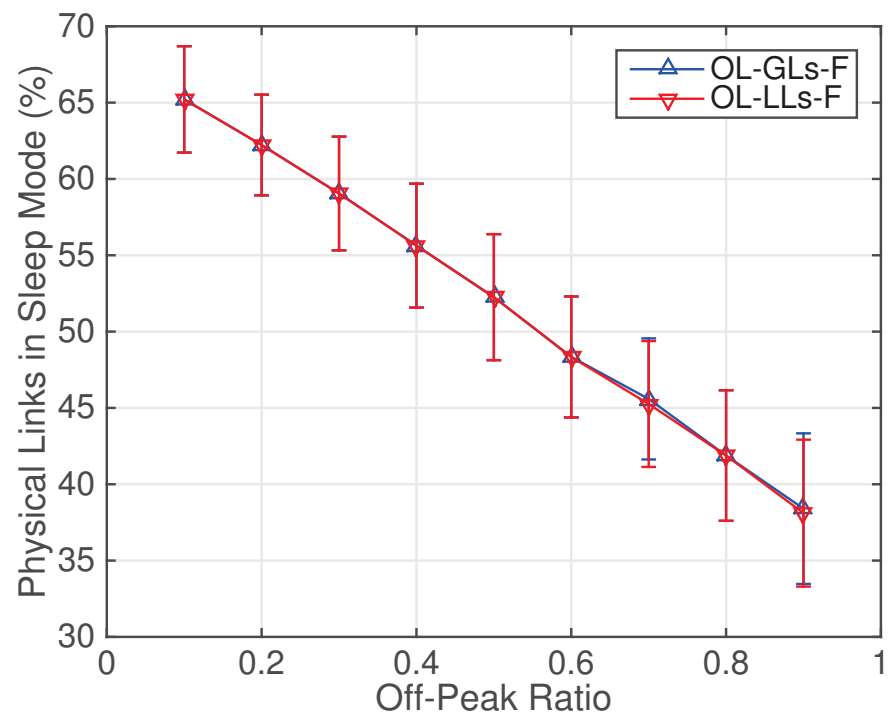

Figure 3.3: Off-peak link energy optimization by global link reconfiguration vs. local link reconfiguration (splittable traffic)

OL-GLs-F is expected to deliver the most optimum level of energy-saving. However, different from OL-GLs-F, OL-LLs-F enables the providers to adjust the level of the reconfiguration and control the possible traffic disruptions. This is possible through the constraint in Equation 3.11 that prevents the local link reconfiguration solution from modifying the allocated traffic capacities in physical links with stress rate larger than a threshold, set by the providers. Although this approach could help 
decreasing the possible traffic disruptions, it needs to provide the possibility of achieving the maximum energy-saving level for the providers. Since the size of generated VNEs are small in a small simulation setup, the stress rate for the majority of the physical links is less than the chosen stress rate threshold of 0.6. Therefore, Figure 3.3 confirms that when the constraint in Equation 3.11 is relaxed, OL-LLs-F could achieve the same energy-saving level as OL-GLs-F.

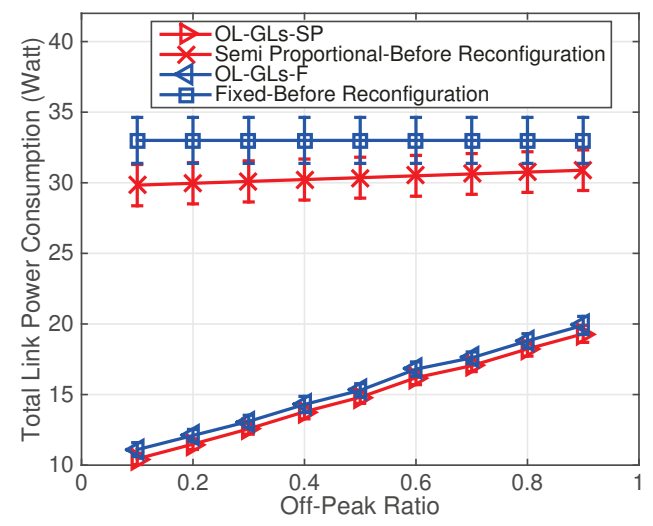

(a)

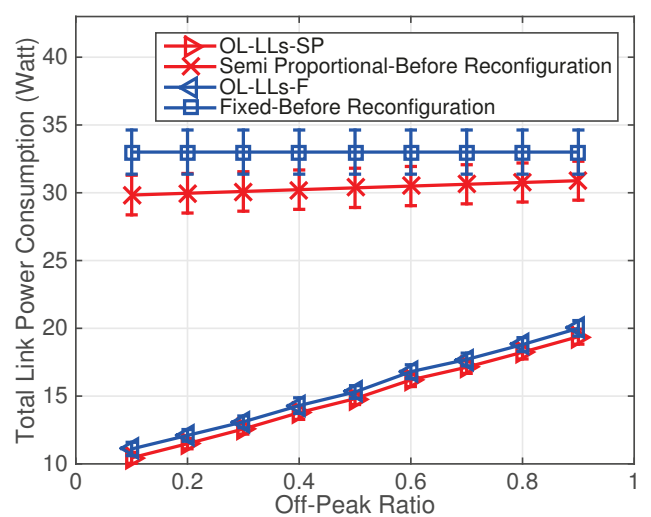

(b)

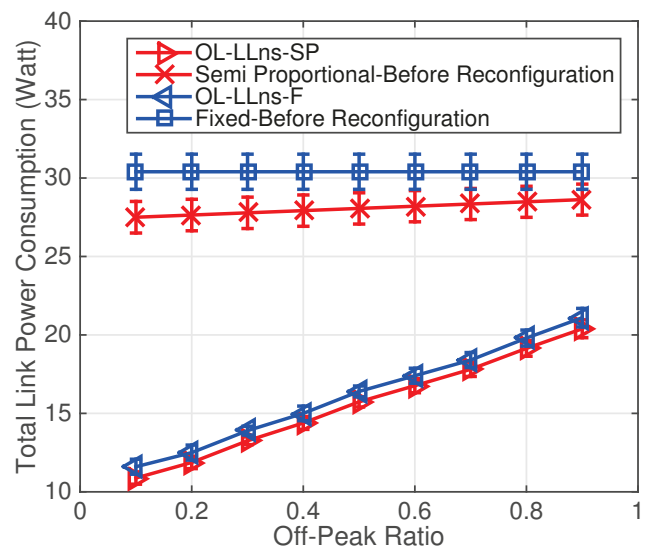

(c)

Figure 3.4: (a) The total link power consumption of off-peak link energy optimization by global link reconfiguration. (b) The total link power consumption of off-peak link energy optimization by local link reconfiguration for splittable traffic. (c) The total link power consumption of off-peak link energy optimization by local link reconfiguration for non-splittable traffic.

In addition, we measured the total link power consumption for both off-peak link 
energy optimization by global link reconfiguration and local link reconfiguration solutions on a small random simulation setup. This measurement has been performed according to both Fixed and Semi-Proportional link power models. The total link power consumption has been measured for the off-peak ratio range of 0.1 to 0.9 , before and after applying the proposed energy-saving solutions. According to [56] and because the random physical links' capacities are generated uniformly in the range of $100-200 \mathrm{Mbps}, P^{b}$ is $0.9 \mathrm{Watt}$ and $P^{m}$ is $1.0 \mathrm{Watt}$ for any physical link. The measurement results for off-peak link energy optimization by global link reconfiguration, and off-peak link energy optimization by local link reconfiguration for splittable and non-splittable traffic, are shown in Figures 3.4a, 3.4b, and 3.4c, respectively.

The results in Figures 3.4a, 3.4b, and 3.4c, demonstrate that all of the formulated programs are able to reduce a VNE's link power consumption, effectively. Considering the results of when Fixed link power model is used, the total link power consumption for any off-peak traffic rate is constant, when no energy-saving solution is employed. Nevertheless, the power consumption is changing with the traffic rate even before applying any energy-saving solution, when Semi-Proportional link power model is used. By applying any of the proposed energy-saving techniques (global/local link reconfiguration), the total link power consumption based on both link power models will be decreased. Note that increasing the off-peak ratio raises the total link power consumption, as a larger number of links will be left activated.

In the same simulation setup, we calculated the percentage of power saved in physical links, by the formulated programs for off-peak link energy optimization by local link reconfiguration problem. This is tested in the case of splittable and nonsplittable traffic, for both Fixed and Semi-Proportional link power models. The results are shown in Figure 3.5. The outcome of programs shows that the rate of power we could save in physical links is decreasing when the off-peak ratio is increasing, because the programs are more limited in terms of finding alternative paths.

Besides, Figure 3.5 illustrates that it is probable to save a higher amount of power when traffic is splittable, in comparison to when traffic is non-splittable. This is because the programs are more flexible in terms of finding alternative paths when they could split the traffic to multiple paths. Moreover, Figure 3.5 confirms that we could reach a higher rate of power-saving when the objective function is formulated according to the Fixed link power model, in comparison to when the objective function is formulated based on Semi-Proportional link power model. This is mainly due 
to two reasons. First, since the power consumption is varying based on the traffic load in Semi-Proportional link power model rather than being a constant amount based on Fixed link power model, a physical link's power consumption in the case of Semi-Proportional link power model is less than when Fixed link power model is employed. Consequently, the amount of saved power with Semi-Proportional link power model might be less than the amount of saved power with Fixed link power model, if the program sets the same physical links into the sleep mode. In addition, if the energy-saving program sets a physical link into the sleep mode, then it needs to find an alternative path to support the off-peak traffic of the removed link. In the case of Semi-Proportional link power model, the rerouted traffic increases the power consumption over the alternative path. However, in the case of Fixed link power model, since traffic load does not affect a link's power consumption, the rerouted traffic does not increase the power consumption over the alternative path.

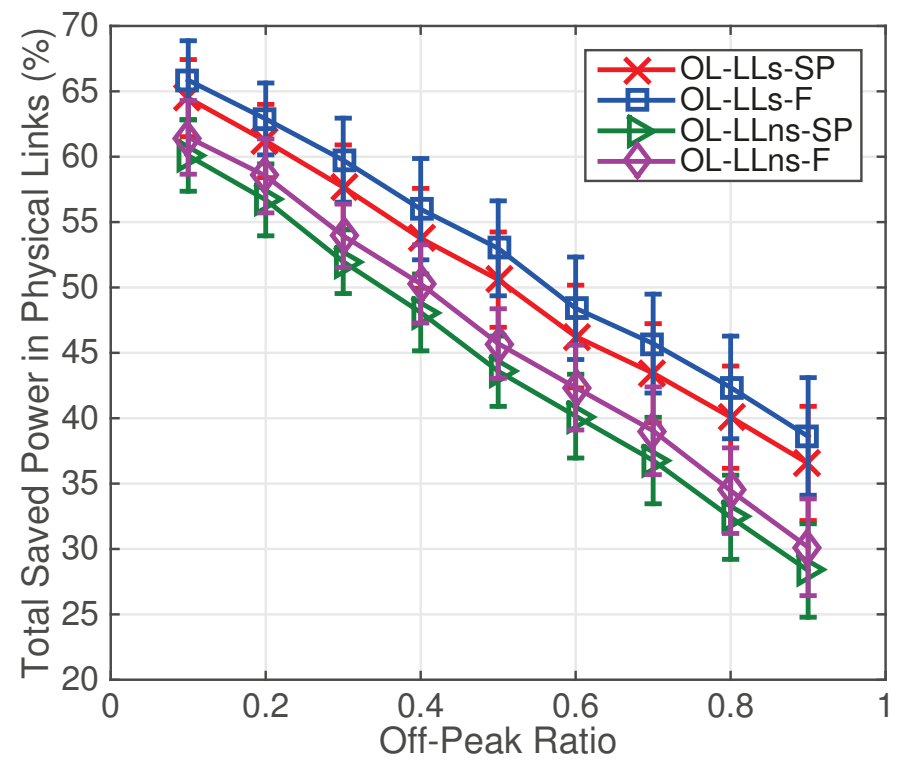

Figure 3.5: The total saved power with off-peak link energy optimization by local link reconfiguration for splittable and non-splittable traffic

\subsubsection{The Heuristic}

We solved the formulated BILP for OL-LLns-F and compared its ability in terms of sleeping physical links to the proposed heuristic for the same problem. The average 
results are measured for the different off-peak traffic ratios on a small random simulation setup, and shown in Figure 3.6. The BILP results set the optimum points, while the heuristic algorithm still reveals reasonable results. However, the heuristic algorithm is considerably simpler and faster in terms of required run time.

It is also important to evaluate the effectiveness of the proposed heuristic algorithm for OL-LLns-F. We assess the ability of the heuristic based on different factors on the defined large random simulation setups.

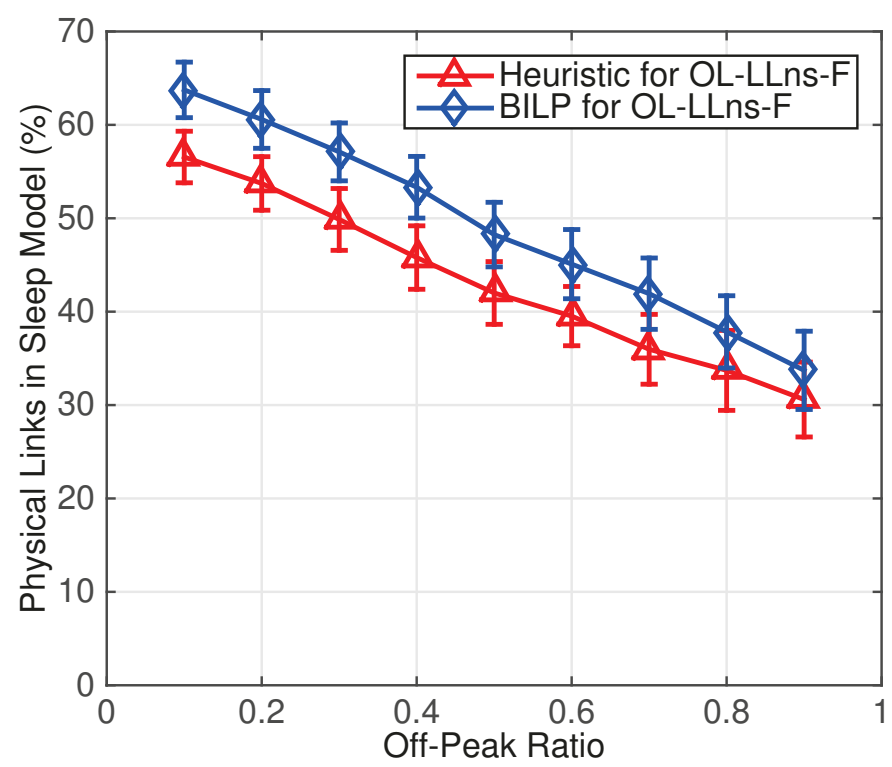

Figure 3.6: The BILP vs. the heuristic for off-peak link energy optimization by local link reconfiguration (non-splittable traffic)

The heuristic is formulated based on Fixed link power model. In this regard, the total link power consumption is measured for the different off-peak ratios, before and after applying the proposed heuristic on a large random simulation setup. The average results are shown in Figure 3.7. The power consumption is constant before applying the heuristic. By applying the heuristic, the total VNE's link power consumption will be reduced. Note that increasing the off-peak ratio raises the total link power consumption, as a larger number of links are left active. 


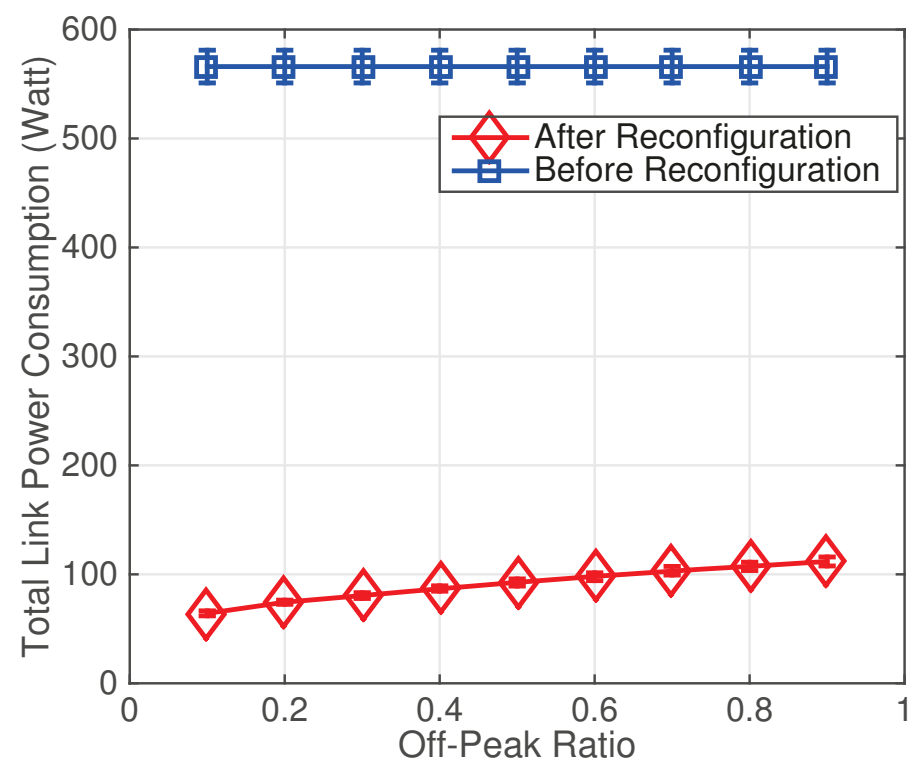

Figure 3.7: The total link power consumption of the heuristic for off-peak link energy optimization by local link reconfiguration

Besides, it is essential to check the ability of the heuristic for different numbers of involved virtual networks, as increasing the number of virtual networks adds several constraints in terms of sleeping a single physical link. The ability of the proposed heuristic on setting physical links into the sleep mode during the off-peak hours, is tested over two large random simulation setups. In the first large random setup, each VNE contains two virtual networks, but in the second large random setup, every VNE includes three virtual networks. The effectiveness of the algorithm is evaluated for the range of off-peak traffic ratios. The average results for both setups are shown in Figure 3.8. Figure 3.8 shows that it is probable to set a notable number of physical links into the sleep mode during the off-peak period, by implementing the suggested heuristic algorithm. For the first setup, when the off-peak traffic ratio is 0.1 , the proposed heuristic sets $89.1230 \%$ of the physical links into the sleep mode. This happens while the reconfiguration heuristic still accommodates the off-peak traffic demands of involved VNs. In addition, Figure 3.8 confirms that mapping an extra virtual network onto the substrate network degrades the ability of heuristic in terms of saving power. This is because the algorithm assesses the allocated traffic capacities to every virtual link in each substrate link in order to find a replaced path. By adding new virtual networks, new virtual links are mapped onto physical links, and therefore 
there are more constraints for the algorithm. Consequently, a smaller number of physical links is capable to be set into the sleep mode over off-peak hours. Moreover, decreasing the off-peak ratio decreases the difference between outcome of the first and second simulation setups. When off-peak traffic rate is low, the programs are more flexible in terms of finding alternative paths. Hence, the energy-saving ability of the programs is less affected by adding extra virtual networks when the off-peak ratio is small, in comparison to when we have large off-peak traffic rates.

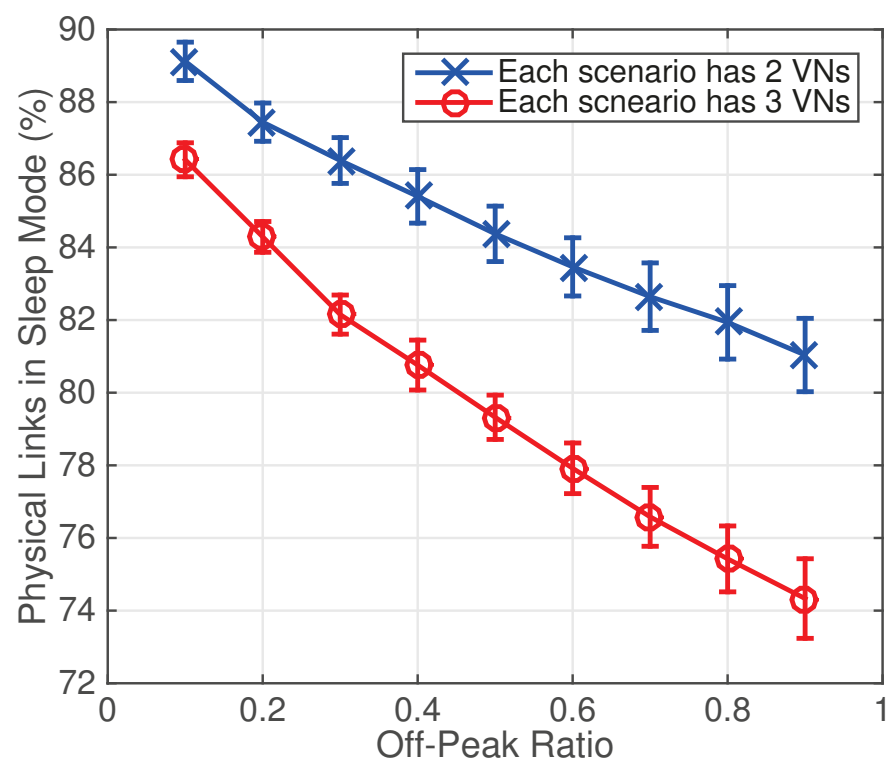

Figure 3.8: The link reconfiguration heuristic for the different numbers of involved VNs

Moreover, it is explained in Section 3.4 that the proposed heuristic, similar to the formulated BILP for OL-LLns-F, does not re-allocate the allocated traffic capacities in physical links with $\tilde{s}\left(l_{s}^{i, j}\right) \geq \mathcal{T}$, in order to decrease the service disruptions due to the reconfiguration. Figure 3.9 studies the effect of changing the stress rate threshold $\mathcal{T}$ on the capability of the heuristic for setting physical links into the sleep mode, over a large random simulation setup. Figure 3.9 shows that decreasing $\mathcal{T}$, decreases the number of physical links the heuristic sets into the sleep mode, because a smaller number of substrate links are considered for energy-saving. Although setting a smaller $\mathcal{T}$ decreases the amount of power the solutions could save, it reduces the traffic interruptions, due to the reconfiguration. Consequently, the providers could control the possible traffic interruptions by adjusting $\mathcal{T}$. Note that because of the specific 
chosen amounts of physical and virtual links' bandwidth capacity in our defined large random simulation setups, the stress rate of the majority of the physical links in the considered simulation setup is less than 0.3. Consequently, the heuristic outcome is almost constant for $\mathcal{T}$ of greater than 0.3 .

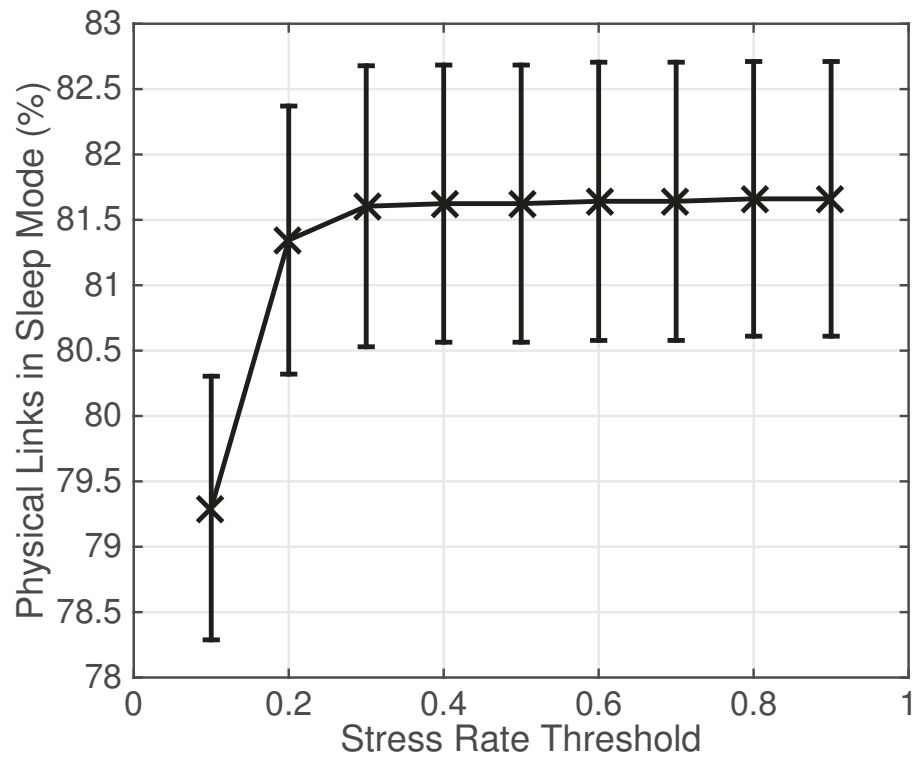

Figure 3.9: The effect of changing the stress rate threshold on the heuristic's outcome

Additionally, the suggested heuristic for OL-LLns-SP is expected to be more effective in comparison to our previous work [70] and other similar studies in [76, 77]. We compared the outcome of the proposed heuristic in this chapter to our previous algorithm in [70], over a large random simulation setup. As the result is clear in Figure 3.10, this link reconfiguration algorithm is able to set a larger number of physical links into the sleep mode over the off-peak time, with the same constraints. This is because the methods in $[70,76,77]$ do not modify the allocation of mapped virtual networks, while they only reroute the traffic to the already allocated traffic capacities to virtual links. Nonetheless, in this chapter, we reconfigure the mapping of virtual links to reach higher power-saving rates. 


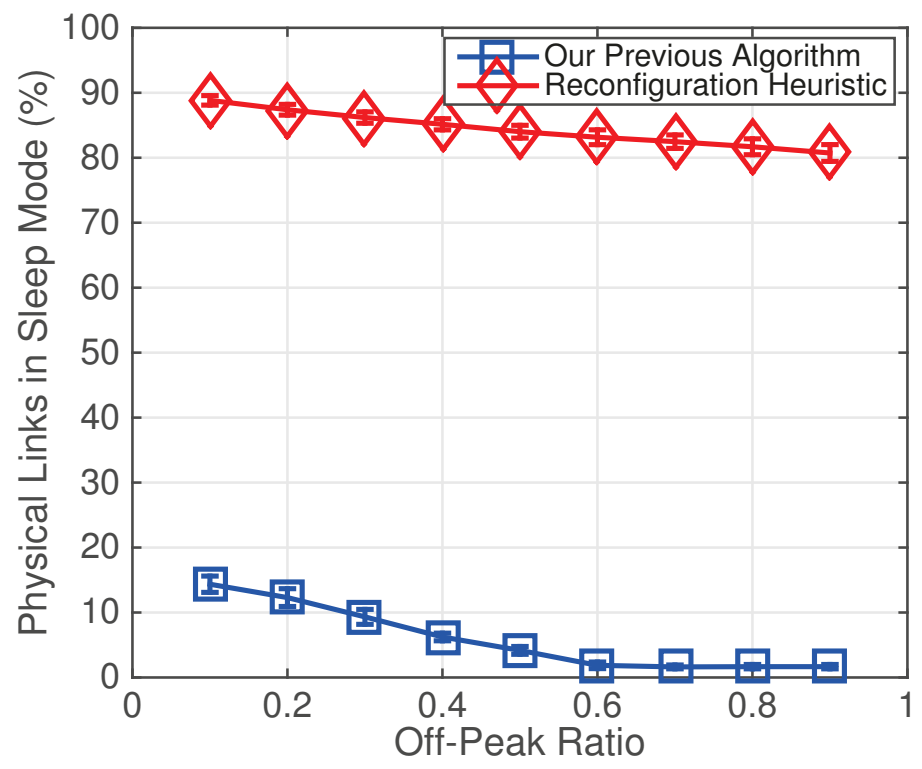

Figure 3.10: The link reconfiguration heuristic vs. our previous algorithm

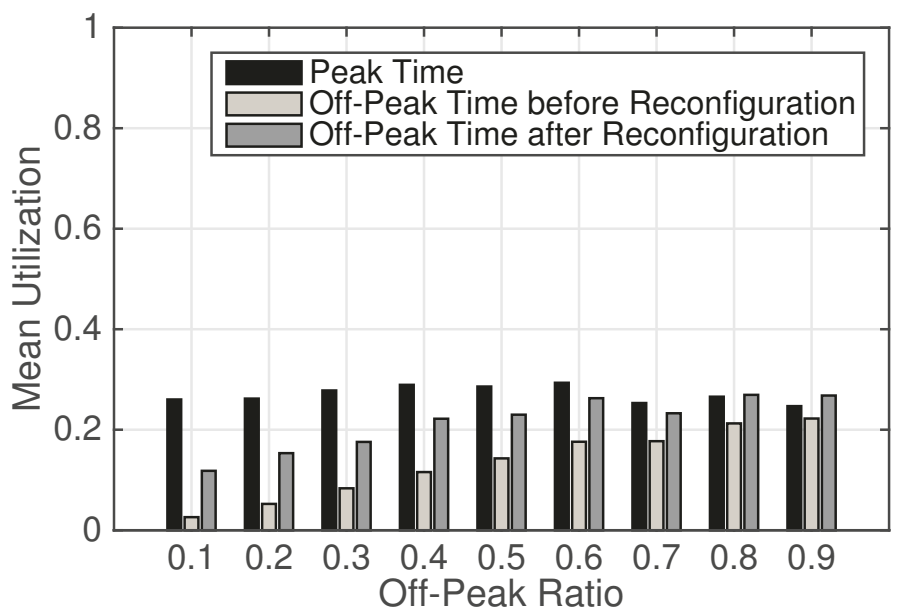

Figure 3.11: The mean link utilization over different configurations

Furthermore, re-allocating the traffic capacities in the other substate links causes changes to the link utilization. Accordingly, it is needed to make sure the increased utilization is controlled and does not cause congestion. The link utilization for three different configurations is tested on a large random simulation setup, and the average results are shown in Figure 3.11. The first configuration is for the peak time when allocated bandwidth is able to handle "Worst-Case" scenarios. The second configuration is for the off-peak period while no energy-saving algorithm is implemented. Over 
this period the links are less utilized while the same bandwidth capacity is allocated and consume the same power as the peak time. After applying our suggested heuristic, the average link utilization is increased, but it is still less than the maximum utilization. Note that the link utilization shown in Figure 3.11 is the mean of the maximum points of all the test scenarios.

It is also necessary to validate the effectiveness of our proposed approach against a real topology. In this regard, we tested the proposed heuristic on a new random simulation setup that contains 10 randomly generated VNEs. Each VNE in this new random simulation setup, has 2 random virtual networks that are mapped onto the GÉANT network topology [89], while every virtual network has 10 nodes. GÉANT has 22 nodes and 36 bidirectional links. We considered GÉANT network as the substrate network, because it is a real universal topology. Figure 3.12 shows the results for this setup. The results confirm that the heuristic is able to effectively reduce the network's link power consumption.

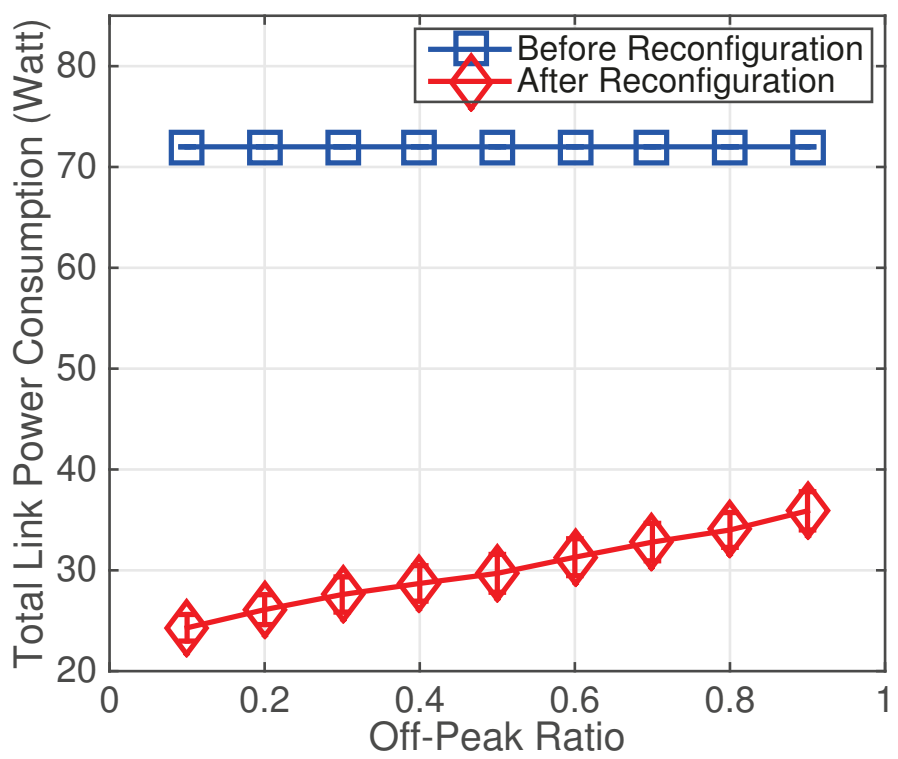

Figure 3.12: The total link power consumption before and after applying the heuristic on the GÉANT simulation setup

All the formulated ILPs in this chapter are $\mathcal{N} \mathcal{P}$-hard, while the complexity of the proposed heuristic algorithm is $\mathcal{O}\left(\left|E_{s}\right|^{3}|\Phi|^{2}\left|E_{v}^{m}\right|^{2}\left(\left|E_{s}\right|+\left|V_{s}\right| \log \left|V_{s}\right|\right)\right)$. So, it is expected the proposed heuristic needs less run time in comparison to the formulated integer linear programs. We verified run time for each method (when the objective 
is defined based on Fixed link power model) on a single small random VNE that has a substrate network and 2 virtual networks, while each has 10 nodes. The run time is measured for each formulated optimization program as well as the heuristic, when the off-peak ratio is 0.5. The results are shown in Figure 3.13. The run time of the MILPs for OL-GLs-F and OL-LLs-F are 215.7 seconds and 3,302.4 seconds, respectively. Besides, the run time of the BILP for OL-LLns-F is 20,322.0 seconds. However, the run time of the heuristic for OL-LLns-F is only 0.0409 seconds, which is considerably small in comparison to the required run time for the formulated BILP of the same problem. The run time for local link reconfiguration BILP for nonsplittable traffic is higher than the MILP of the same problem for splittable traffic. This is because the BILP needs to find an alternative path for every allocated traffic capacity in substrate links, while the MILP has to find an alternative path for every bundled traffic capacity. Therefore, the BILP for non-splittable traffic has more constraints than the MILP for splittable traffic. In addition, the run time of local link reconfiguration MILP for splittable traffic is larger than the run time for global link reconfiguration MILP, since the local link reconfiguration is more complex and has a larger number of constraints than the global link reconfiguration.

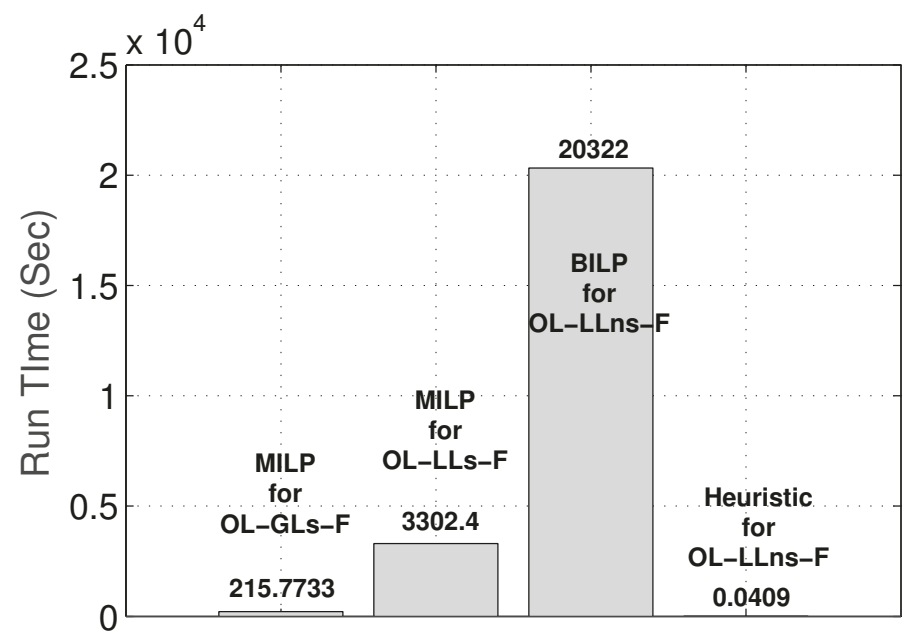

Figure 3.13: The run time for different methods

The simulation results prove the suggested energy-saving solutions are able to reduce a VNE's link power consumption, during the off-peak period, effectively. Besides, the proposed heuristic is a simple and fast algorithm that works closely to the 
optimum points. Note that every simulation setup is quite large to cover a substantial number of random topologies in order to verify the effectiveness of the proposed solutions. Besides, the calculated confidence intervals confirm that the results are precise enough to reveal significances of suggested energy-saving methods.

\subsection{Summary}

In this chapter, we discussed multiple novel energy-saving solutions that globally/locally optimize a VNE's link energy consumption, during the off-peak time. The proposed fine-grained local reconfiguration enables the providers to adjust the level of the reconfiguration, and accordingly control the possible traffic disruptions. An Integer Linear Program (ILP) is formulated for each problem. Since the ILPs are $\mathcal{N} \mathcal{P}$-hard, a novel heuristic algorithm is also proposed. Simulation results show that the energy-saving solutions are noticeably effective and the heuristic achieves closely to the optimum points. Because physical nodes are also essential energy consumers in VNEs, it is necessary to develop energy-saving techniques that minimize both node and link energy consumption in a VNE. This will be discussed in the next chapter. 


\section{Chapter 4}

\section{Off-Peak Energy Optimization for Nodes and Links in a VNE}

\subsection{Introduction}

In Chapter 3 we studied multiple energy-saving solutions for the off-peak period of a VNE. The provided solutions are developed to reduce only a VNE's link energy consumption. However, physical nodes are also essential energy consumers in VNEs. According to the discussed power models in Section 2.3, physical nodes normally consume larger amounts of power than physical links. Consequently, it is necessary to develop energy-saving techniques that optimize both node and link energy consumption in a VNE.

In this chapter, we extend our previous study in Chapter 3, and discuss multiple novel energy-saving solutions that optimize a VNE's node and link energy consumption during the off-peak time, according to two defined power models. We approach the problem by reconfiguring the mapping for some of the already embedded virtual nodes and links. We assume that substrate and virtual networks are homogeneous. All the substrate and virtual nodes are assumed to be switches/routers to reflect the network environment. This is the case in the majority of existing related research studies.

In this regard, first, we formulated a Mixed Integer Linear Program (MILP) for off-peak node and link energy optimization by local node and link reconfiguration. A stress rate is defined for substrate nodes. Accordingly, the MILP may set less stressed substrate nodes and their adjacent substrate links into the sleep mode for the off-peak time. We re-map a virtual link if and only if we sleep the substrate node 
that hosts its source/sink virtual node, or at least one substrate node that relays its traffic over its embedded path. The energy consumption of a re-mapped virtual link is also minimized.

Because reconfiguring the mapping for both of virtual nodes and virtual links may be expensive and cause the interruptions to the normal network operations, we come up with another methodology that optimizes node and link energy consumption of a VNE, during the off-peak time, by reconfiguring the mapping for only some of the virtual links. We do not reconfigure the mapping of the embedded virtual nodes in this case. We call the latter problem off-peak node and link energy optimization by local link reconfiguration. We define a different stress rate for intermediate substrate nodes, which do not host a virtual node and only relay the traffic. Accordingly, a solution is proposed to optimize the total energy consumption of the intermediate substrate nodes and substrate links during the off-peak time. This method might set the less stressed intermediate substrate nodes and their respective substrate links into the sleep mode for the off-peak time. We re-map a virtual link if and only if we sleep at least one intermediate substrate node over its embedded path. The energy consumption of a re-mapped virtual link is also minimized. An MILP for splittable traffic and a Binary Integer Linear Program (BILP) for non-splittable traffic are formulated for this strategy.

These methods enable the providers to change the level of the reconfiguration by adjusting the stress rate's threshold. Therefore, they can control the possible traffic interruptions of the reconfiguration. Clearly, there is a trade-off between the energy-saving level and the possible traffic interruptions.

Since the formulated optimization programs are $\mathcal{N} \mathcal{P}$-hard, we also suggest a heuristic algorithm for off-peak node and link energy optimization by local link reconfiguration. The simulation results confirm that the heuristic algorithm can achieve closely to the optimum points set by the formulated optimization program, while it is considerably faster. The proposed heuristic is scalable to large network sizes.

The related works are discussed in Section 2.3.3. In this regard, our contributions in this chapter are:

- We propose a local optimization program in the form of an MILP that reconfigures the mapping of both virtual nodes and links during the off-peak time, to minimize the total energy consumption of nodes and links in a VNE in that period. 
- We propose local optimization programs in form of ILPs that reconfigure the mapping of virtual links during the off-peak time, to minimize the energy consumption of intermediate nodes and links in a VNE in that period.

- The solutions are formulated according to two power models.

- Different programs are formulated for splittable and non-splittable traffic in order to study the impact of traffic splittablity.

- We present a scalable heuristic algorithm that can achieve closely to the optimum results, but considerably faster than the optimization program.

- We define different stress rates for distinct types of substrate nodes. Therefore, different from any related research studies, the proposed methods enable the providers to control the level of reconfiguration and the possible traffic interruptions.

- Different from previous research studies [17,60,62,64,69], our method does not decrease the network admittance ratio for new virtual networks. This is because we reconfigure the mapping of the already accepted VNs only for the off-peak period, and they could be reconfigured back to their peak the mapping in the case of an unexpected new demand.

- Our solutions are not limited to a sub-topology as the case in [70], and so they have a larger degree of freedom to save the energy.

- We do not move VMs, so our method does not have the difficulties of [16].

- We assess the impact of different parameters on the energy-saving capability of the discussed solutions, through extensive simulations.

- To the best of our knowledge, these problems are not defined and formulated mathematically in any published study.

This chapter is organized as follows: Two power models for physical nodes and links are reviewed in Section 4.2. Multiple optimization programs are defined and formulated as ILPs in Section 4.3 and the suggested heuristic is discussed in Section 4.4. In order to evaluate the performance of the ILPs as well as the heuristic algorithm, multiple random scenarios have been simulated over randomly generated VNEs and the results are analyzed in Section 4.5. The chapter will conclude in Section 4.6. 


\subsection{Power Models}

Various node and link power models are reviewed in Sections 2.3.1 and 2.3.2, respectively. In this chapter, we assume that substrate networks are homogeneous. All the substrate nodes are assumed to be switches/routers to reflect the network environment. Note that the proposed solutions are general, and they could also employ physical server power models. We discuss our energy-saving solutions according to Fixed power model and Semi-Proportional power model.

Equation 2.45 defines the actual power consumption $\tilde{p}\left(v_{s}^{i}\right)$ for a physical node $v_{s}^{i}$, based on Fixed power model. The actual power consumption $\tilde{p}\left(l_{s}^{i, j}\right)$ of a physical link $l_{s}^{i, j}$ can be found by Equation 2.58, according to Fixed power model.

In addition, Equation 2.46 defines the actual power consumption $\tilde{p}\left(v_{s}^{i}\right)$ of a substrate node $v_{s}^{i}$ according to Semi-Proportional power model. Similar to the node power model, Equation 2.59 defines the actual power consumption $\tilde{p}\left(l_{s}^{i, j}\right)$ of a physical link $l_{s}^{i, j}$ based on Semi-Proportional power model.

Because switches/routers are considered as the physical nodes, $\tilde{p}^{b}\left(v_{s}^{i}\right)$ and $\tilde{p}^{m}\left(v_{s}^{i}\right)$ can be determined by Equation 4.1 and Equation 4.2, respectively [56,57]. $\tilde{p}^{b}\left(v_{s}^{i}\right)$ and $\tilde{p}^{m}\left(v_{s}^{i}\right)$ for a server physical node could be found through calibration experiments as described in [59].

$$
\begin{gathered}
\tilde{p}^{b}\left(v_{s}^{i}\right)=0.85 C_{b}\left(v_{s}^{i}\right)^{\frac{2}{3}} \\
\tilde{p}^{m}\left(v_{s}^{i}\right)=C_{b}\left(v_{s}^{i}\right)^{\frac{2}{3}}
\end{gathered}
$$

Consequently, $\tilde{p}\left(v_{s}^{i}\right)$ for the case of Semi-Proportional power model could be rewritten as Equation 4.3.

$$
\tilde{p}\left(v_{s}^{i}\right)=0.85 \alpha\left(v_{s}^{i}\right) C_{b}\left(v_{s}^{i}\right)^{\frac{2}{3}}+\frac{0.15 r\left(v_{s}^{i}\right)}{C_{b}\left(v_{s}^{i}\right)^{\frac{1}{3}}}
$$

In addition, $\tilde{p}^{b}\left(l_{s}^{i, j}\right)$ and $\tilde{p}^{m}\left(l_{s}^{i, j}\right)$ for a physical link are depended to the physical link bandwidth capacity $C_{b}\left(l_{s}^{i, j}\right)$, its length, and the cable type.

Today's networks are designed based on Fixed power model, so it is a common model that is widely used [56]. Nevertheless, it is not efficient that an active physical node or link consumes a constant amount of power, regardless of its traffic load. Therefore, the physical nodes and links are expected to get modified, so their power 
consumption will be more adaptive to their traffic load. The Semi-Proportional power model brings a traffic-adaptive power model that formulates the power consumption of a physical node or link according to its traffic load.

A power model determines the energy-saving methodology. In this chapter, we assume all the substrate links are in the same range of bandwidth capacity, so they consume the same amount of power. First, considering Fixed power model, a physical node or link consumes a constant amount of power, regardless of the traffic load. Therefore, the best energy-saving strategy to minimize a physical node or link's energy consumption is setting the device into the sleep mode. However, because physical nodes consume considerably a larger amount of power in comparison to physical links, it is more important to set a physical node into the sleep mode, rather than a physical link. Second, in the case of Semi-Proportional power model, the traffic load in a device changes its power consumption. Although a large portion of power is still consumed to keep the device operational, it might be possible to save some energy by rerouting the device's traffic to other active nodes and links with the larger value for the division of the difference between the maximum and the base power consumption by the bandwidth capacity. So, in the case of Semi-Proportional power model it may be feasible to save energy by sleeping a network element, or rerouting the traffic. Consequently, the objective for an energy-saving solution based on Fixed power model is different to the objective for an energy-saving method based on Semi-Proportional power model. In this regard, we formulate multiple node and link energy-saving solutions according to the both power models.

\subsection{Integer Linear Programs}

During the off-peak time of a VNE, the virtual networks' demands in terms of traffic, processing, etc., are decreased. Accordingly, it might be achievable to save notable amounts of energy in substrate nodes and links, by reconfiguring the mapping of the already allocated resources, while still satisfying the off-peak demands. In this regard, we define a general reconfiguration problem that targets mapping of both physical nodes and links to minimize a VNE's energy consumption during the off-peak period. The problem description is the followings:

Given:

- A homogeneous substrate network topology 
- Allocated virtual networks' topologies

- For each substrate node: The physical bandwidth capacity, and the processing capacity, as well as the allocated processing capacity to each virtual node in the substrate node

- For each substrate link: The physical bandwidth capacity, as well as the allocated bandwidth capacity to every virtual link in the substrate link

- Off-peak traffic and processing demands of each virtual network, provided by virtual network customers, or network traffic prediction techniques

Find:

- The modified off-peak VNE mapping that leads to the minimum total node and link energy consumption during the off-peak time

Constraints:

- Supporting the off-peak traffic and processing demands

We approach the defined energy-saving problem with two different local reconfiguration strategies. First, we reconfigure the mapping for some of the already allocated virtual nodes and links, during the off-peak period, to minimize the total node and link energy consumption of the network in that period. It is possible to minimize a VNE's total energy consumption by setting any capable physical node or link into the sleep mode, or rerouting its traffic load. Rerouting the traffic may also help saving the energy. However, we still need to accommodate the demanded off-peak processing and traffic rates. We call this problem off-peak node and link energy optimization by local node and link reconfiguration.

Reconfiguring the mapping for both of virtual nodes and links might be expensive or cause the interruptions to the normal network operations. Besides, some times it is infeasible to reconfigure the mapping of virtual nodes. For example, moving an embedded access/edge virtual node to the other part of a substrate network might be impossible. An access/edge virtual node connects a VN to other networks (on different substrate networks). Hence, we come up with the second reconfiguration method that optimizes total node and link energy consumption in a VNE during the off-peak time, by reconfiguring the mapping for only some of the already allocated 
virtual links. The second method does not reconfigure the already allocated virtual nodes, and therefore it is not possible to sleep the physical nodes that one or multiple virtual nodes are allocated in them. However, it is probable to save the energy in the capable intermediate substrate nodes, or any qualified substrate link. Rerouting traffic load over the network may also help to save energy based on some power models. We call the second problem off-peak node and link energy optimization by local link reconfiguration.

Both of the reconfiguration strategies are local optimization problems, as they do not reconfigure the mapping for all of the virtual nodes or links. This helps to reduce the possible interruptions to the network operations. Although the second reconfiguration strategy does not reconfigure virtual nodes and therefore it is expected to save smaller amounts of energy in comparison to the first method, it causes fewer interruptions to the normal network operations.

Note that virtual network customers might have multiple constraints for their demanded virtual nodes and links. In this chapter, we concern only with the requested switching and processing capacity for switches/routers, and the traffic demands.

In this regard, first, we model a VNE mathematically. Afterwards, for both Fixed and Semi-Proportional power models, we formulate integer linear programs for offpeak node and link energy optimization by local node and link reconfiguration, and off-peak node and link energy optimization by local link reconfiguration. Since the traffic type (splittable/non-splittable) has a major impact on the solution's methodology, we formulate off-peak node and link energy optimization by local link reconfiguration for both splittable and non-splittable traffic.

\subsubsection{The Network Model}

A substrate network is modeled similar to the substrate network model in Section 3.3.1. $C_{c}\left(v_{s}^{i}\right)$ expresses the processing capacity of $v_{s}^{i}$.

The $n$th virtual network is also modeled similar to Section 3.3.1. In addition, $v_{n}^{k}$ refers to the $k$ th virtual node in the $n$th virtual network. $\dot{C}_{c}\left(v_{n}^{k}\right)$ represents off-peak processing demand of $v_{n}^{k}$.

In a VNE embedding procedure, the $n$th requested virtual network $G_{n}$ is mapped onto a defined substrate network $G_{s}: G_{n} \rightarrow G_{s}$. Virtual nodes are allocated in the chosen physical nodes. $\phi\left(v_{n}^{k}, v_{s}^{i}\right)$ indicates whether a virtual node $v_{n}^{k}$ is allocated in a physical node $v_{s}^{i}$, or not. $\phi\left(v_{n}^{k}, v_{s}^{i}\right)$ is 1 , if $v_{n}^{k}$ is allocated in $v_{s}^{i}$. Otherwise, it is 0 . 
A virtual link could be mapped onto a single physical link, or multiple physical links that makes a physical path. If traffic is splittable, then a virtual link could be mapped onto multiple substrate paths. However, if traffic is non-splittable, then each traffic demand is carried by only one path. The allocated virtual links of the $n$th VN are presented as the set of ordered allocated virtual node pairs $\left(a_{m}, b_{m}\right), m=1,2, \ldots, L_{n}$. $l_{n}^{a_{m}, b_{m}}$ represents the $m$ th virtual link, belonging to the $n$th $\mathrm{VN}$, that connects the virtual node mapped onto the $a_{m}$ th substrate node to the virtual node mapped onto the $b_{m}$ th substrate node. $r_{n}^{m}$ stands for the off-peak traffic demand of $l_{n}^{a_{m}, b_{m}}$. In addition, $r_{n}^{i, j}(m)$ denotes the off-peak traffic demand of the allocated traffic capacity to $l_{n}^{a_{m}, b_{m}}$ in $l_{s}^{i, j}$. During the off-peak period, the reserved processing and traffic capacity for virtual nodes and links in physical nodes and links, respectively, are equal to their particular off-peak demands, and the rest of the physical capacity could be shared.

\subsubsection{Programs Based on Fixed Power Model}

In this section, we develop the energy-saving solutions based on Fixed power model. It is assumed that all the substrate links are in the same range of the bandwidth capacity. Conforming to Fixed power model, the best energy-saving strategy to reduce a physical node or link's energy consumption is setting the device into the sleep mode. The following energy-saving methods are formulated according to this logic.

\section{Off-peak Node and Link Energy Optimization by Local Node and Link Reconfiguration (ONL-LNLs-F)}

We define a stress rate for a substrate node. Accordingly, an MILP is formulated for the problem. The MILP may set less stressed substrate nodes and their adjacent substrate links into the sleep mode for the off-peak time. We re-map a virtual link if and only if we sleep the substrate node that hosts its source/sink virtual node, or at least one substrate node that relay its traffic over its embedded path. The program also sets the maximum number of physical links into the sleep mode during the reallocation process of virtual links. This problem is defined according to Fixed power model. The traffic is assumed to be splittable in this problem to deliver the highest level of energy-saving. We call this problem ONL-LNLs-F.

In this problem, the stress rate $\tilde{s}_{1}\left(v_{s}^{i}\right)$ of a physical node $v_{s}^{i}$ reveals the intensity of total off-peak processing and traffic demands in the node. Equation 4.4 defines $\tilde{s_{1}}\left(v_{s}^{i}\right)$. 


$$
\begin{array}{r}
\tilde{s_{1}}\left(v_{s}^{i}\right)=\frac{\sum_{\left\{n \mid G_{n} \in \Phi\right\}} \sum_{k \in V_{n}} \phi\left(v_{n}^{k}, v_{s}^{i}\right) \dot{C}_{c}\left(v_{n}^{k}\right)}{C_{c}\left(v_{s}^{i}\right)}\left(\frac{\sum_{\left\{j \mid(i, j) \in E_{s}\right\}} \sum_{\left\{n \mid G_{n} \in \Phi\right\}} \sum_{m=1}^{L_{n}} \dot{r}_{n}^{i, j}(m)}{C_{b}\left(v_{s}^{i}\right)}\right. \\
\left.+\frac{\sum_{\left\{j \mid(j, i) \in E_{s}\right\}} \sum_{\left\{n \mid G_{n} \in \Phi\right\}} \sum_{m=1}^{L_{n}} r_{n}^{j, i}(m)}{C_{b}\left(v_{s}^{i}\right)}\right)
\end{array}
$$

$\tilde{s_{1}}\left(v_{s}^{i}\right)$ considers two parameters. The first parameter is the fraction of the total off-peak processing demands of allocated virtual nodes in a physical node, over the processing capacity of the physical node. The second parameter considers the offpeak traffic by finding the fraction of the total off-peak traffic passing a physical node, over the physical bandwidth capacity of the node. Note that, in this chapter, we assume that the total traffic in a substrate node is the total traffic in its incoming physical links plus the total traffic in its outgoing physical links. This also defines the switching capacity of a substrate node. Accordingly, $\tilde{s_{1}}\left(v_{s}^{i}\right)$ considers the offpeak traffic intensity by considering both incoming and outgoing off-peak traffic to a substrate node. However, if a node is the source/sink of traffic, then it considers only one of the the flows, as the other one is zero.

Consequently, in this problem, a higher physical node's stress rate means the node is more utilized in terms of off-peak processing and traffic demands. In this regard, we do not re-map a virtual node that is mapped onto a physical node with $\tilde{s_{1}}\left(v_{s}^{i}\right) \geq \mathcal{T}$, in order to decrease the traffic disruptions due to the reconfiguration. $\mathcal{T}$ is the stress rate's threshold, and it is a real number between 0 and 1 . The providers can adjust $\mathcal{T}$. Decreasing the value of $\mathcal{T}$ degrades the amount of energy the programs could save, but also reduces the traffic interruptions due to the reconfiguration. This is because a smaller number of physical nodes and links are considered for energy-saving.

We formulate ONL-LNLs-F as an MILP. ONL-LNLs-F is a combination of two sub-problems with the same objective. The first sub-problem is a node reconfiguration problem that reconfigures the mapping of virtual nodes. The second sub-problem is a link reconfiguration problem that reconfigures the mapping of virtual links. The link reconfiguration sub-problem could be formulated as a multi-commodity flow problem. In the context of this sub-problem, a virtual link is a commodity. Multiple virtual links are multiple commodities. In order to achieve the most optimum result, it is required to formulate a single problem that combines both of the sub-problems together. 
The substrate network is known to the program, and therefore the bandwidth capacity of every physical node and link, as well as the processing capacity of each physical node are given. The allocated virtual networks are also identified, so $\phi\left(v_{n}^{k}, v_{s}^{i}\right)$ for any virtual node in every physical node is specified. In addition, the off-peak processing demand $\dot{C}_{c}\left(v_{n}^{k}\right)$ of every virtual node is known to the program. In the context of link reconfiguration sub-problem, we have $L_{n}$ commodities for the $n$th virtual network. For each $l_{n}^{a_{m}, b_{m}}$, as a commodity, we are given a scalar $r_{n}^{m}$ that refers to the virtual link's off-peak traffic demand. Besides, $r_{n}^{i, j}(m)$ for every virtual link in each physical link is specified. Moreover, $\varrho_{n}^{\prime m}\left(v_{s}^{i}\right)$ is 1 , if $v_{s}^{i}$ is a relay substrate node for $l_{n}^{a_{m}, b_{m}} \cdot v_{s}^{i}$ is a relay node for $l_{n}^{a_{m}, b_{m}}$, if source or sink virtual node of $l_{n}^{a_{m}, b_{m}}$ is not mapped onto $v_{s}^{i}$, while $v_{s}^{i}$ is on the allocated path for $l_{n}^{a_{m}, b_{m}}$ and forwards its traffic. Otherwise, $\varrho_{n}^{\prime m}\left(v_{s}^{i}\right)$ is 0 .

Therefore, ONL-LNLs-F is formulated as an MILP as follows:

\section{Optimization Variables:}

- $\alpha\left(v_{s}^{i}\right)$ is a binary variable. It denotes the status of $v_{s}^{i} \cdot \alpha\left(v_{s}^{i}\right)$ is 1 in the case $v_{s}^{i}$ is active, otherwise it is 0 .

- $\alpha\left(l_{s}^{i, j}\right)$ is a binary variable. It refers to the status of $l_{s}^{i, j} \cdot \alpha\left(l_{s}^{i, j}\right)$ is 1 in the case $l_{s}^{i, j}$ is active, otherwise it is 0.

- $\tilde{\phi}\left(v_{n}^{k}, v_{s}^{i}\right)$ is a binary variable that expresses whether a virtual node $v_{n}^{k}$ is reallocated in a physical node $v_{s}^{i}$, during the reconfiguration process, or not. If the program re-allocates $v_{n}^{k}$ in $v_{s}^{i}$, then $\tilde{\phi}\left(v_{n}^{k}, v_{s}^{i}\right)$ is 1 . Otherwise, $\tilde{\phi}\left(v_{n}^{k}, v_{s}^{i}\right)$ is 0 .

- $f_{n}^{\prime i, j}(m)$ is a real-valued variable. It denotes the re-allocated off-peak traffic capacity to the $m$ th virtual link of the $n$th virtual network, in $l_{s}^{i, j} \cdot f_{n}^{i, j}(m)$ is the result of the node reconfiguration sub-problem.

- $\dot{\omega}_{n}^{m}$ is a binary variable. It is 1 , if the program sets at least a relay node for $l_{n}^{a_{m}, b_{m}}$ into the sleep mode. Otherwise, it is 0 .

- $\ddot{\omega}_{n}^{m}$ is a binary variable. It is 1 , if the program re-allocates the source virtual node $v_{n}^{a_{m}}$, or the sink virtual node $v_{n}^{b_{m}}$ of $l_{n}^{a_{m}, b_{m}}$, or both of them to another physical node. Otherwise, it is 0 . 
- $f_{n}^{i, j}(m)$ is a real-valued variable. It denotes the re-allocated off-peak traffic capacity to the $m$ th virtual link of the $n$th virtual network, in $l_{s}^{i, j} \cdot f_{n}^{i, j}(m)$ is the result of link reconfiguration sub-problem.

Objective Function: This program intends to minimize VNE's total node and link energy consumption, during the off-peak time, according to Fixed power model. Equation 4.5 maintains this objective.

$$
\text { Minimize }\left\{\sum_{i \in V_{s}} \alpha\left(v_{s}^{i}\right) \tilde{p}^{m}\left(v_{s}^{i}\right)+\sum_{(i, j) \in E_{s}} \alpha\left(l_{s}^{i, j}\right) \tilde{p}^{m}\left(l_{s}^{i, j}\right)\right\}
$$

Constraints: A substrate node might host virtual nodes, and may also relay the traffic of other virtual nodes. In this regard, setting a substrate node into the sleep mode requires satisfying some constraints to maintain virtual networks' off-peak processing and traffic demands. In regard to the node reconfiguration sub-problem, if the program decides to set a physical node $v_{s}^{i}$ into the sleep mode, then it needs to suggest an alternative physical node for each of the allocated virtual nodes in $v_{s}^{i}$. Equation 4.6 preserves this condition. If $v_{n}^{k}$ is already allocated in $v_{s}^{i}$, then $\phi\left(v_{n}^{k}, v_{s}^{i}\right)$ is 1 . In the case the program sets $v_{s}^{i}$ into the sleep mode, $\alpha\left(v_{s}^{i}\right)$ is 0 . Therefore, $\sum_{j \in V_{s}} \tilde{\phi}\left(v_{n}^{k}, v_{s}^{j}\right)$ must be 1 , which means $v_{n}^{k}$ is re-allocated in an alternative physical node. This constraint also ensures the program re-allocates a virtual node only in a single alternative physical node. However, if $v_{n}^{k}$ is not allocated in $v_{s}^{i}$, then $\phi\left(v_{n}^{k}, v_{s}^{i}\right)$ is 0 , and therefore the constraint in Equation 4.6 is always satisfied. Note that if the program leaves $v_{s}^{i}$ active, then none of the allocated virtual nodes in $v_{s}^{i}$ could be re-allocated.

$$
\phi\left(v_{n}^{k}, v_{s}^{i}\right)\left(1-\alpha\left(v_{s}^{i}\right)-\sum_{j \in V_{s}} \tilde{\phi}\left(v_{n}^{k}, v_{s}^{j}\right)\right)=0, \quad \forall i \in V_{s}, \forall n \in\left\{n \mid G_{n} \in \Phi\right\}, \forall k \in V_{n}
$$

An alternative physical node needs to have some specifications. First, it has to support the off-peak processing demand $\dot{C}_{c}\left(v_{n}^{k}\right)$ of $v_{n}^{k}$. In this regard, as stated in the constraint in Equation 4.7, the physical processing capacity $C_{c}\left(v_{s}^{i}\right)$ of every substrate node must be equal or greater than the total off-peak processing rate of already allocated and not re-mapped virtual nodes in the substrate node $\left(\sum_{\left\{n \mid G_{n} \in \Phi\right\}} \sum_{k \in V_{n}} \phi\left(v_{n}^{k}, v_{s}^{i}\right) \dot{C}_{c}\left(v_{n}^{k}\right)\right)$, plus the total off-peak processing demand of re-allocated virtual nodes in the substrate 
node $\left(\sum_{\left\{n \mid G_{n} \in \Phi\right\}} \sum_{k \in V_{n}} \tilde{\phi}\left(v_{n}^{k}, v_{s}^{i}\right) \dot{C}_{c}\left(v_{n}^{k}\right)\right)$.

$$
\sum_{\left\{n \mid G_{n} \in \Phi\right\}} \sum_{k \in V_{n}} \phi\left(v_{n}^{k}, v_{s}^{i}\right) \dot{C}_{c}\left(v_{n}^{k}\right)+\sum_{\left\{n \mid G_{n} \in \Phi\right\}} \sum_{k \in V_{n}} \tilde{\phi}\left(v_{n}^{k}, v_{s}^{i}\right) \dot{C}_{c}\left(v_{n}^{k}\right) \leq C_{c}\left(v_{s}^{i}\right), \quad \forall i \in V_{s}
$$

Second, the replaced physical node must be active. The constraint in Equation 4.8 retains this concern. If $v_{s}^{i}$ was set into the sleep mode, then $\alpha\left(v_{s}^{i}\right)$ is 0 , and therefore $\sum_{\left\{n \mid G_{n} \in \Phi\right\}} \sum_{k \in V_{n}} \tilde{\phi}\left(v_{n}^{k}, v_{s}^{i}\right)$ must be 0 , which means no virtual node can be re-allocated in $v_{s}^{i}$. This constraint, together with the constraint in Equation 4.6, ensures a virtual node could not be re-allocated in the original substrate node, which is the physical node that the virtual node was previously allocated in. Note that $B_{1}, B_{2}, B_{3}, B_{4}$, and $B_{5}$ are integer numbers, while they must be large enough to be greater than the left hand side of their respective equations.

$$
\sum_{\left\{n \mid G_{n} \in \Phi\right\}} \sum_{k \in V_{n}} \tilde{\phi}\left(v_{n}^{k}, v_{s}^{i}\right) \leq B_{1} \alpha\left(v_{s}^{i}\right), \quad \forall i \in V_{s}
$$

Third, each virtual node is associated with one or multiple virtual links. Hence, as indicated in the constraints presented in Equation 4.9, we do not re-allocate the source virtual node $v_{n}^{a_{m}}$ and the sink virtual node $v_{n}^{b_{m}}$ of the $m$ th virtual link in a single substrate node. This is the case in the majority of the available VNE embedding methods.

$$
\begin{gathered}
\tilde{\phi}\left(v_{n}^{a_{m}}, v_{s}^{i}\right)+\tilde{\phi}\left(v_{n}^{b_{m}}, v_{s}^{i}\right) \leq 1, \\
\phi\left(v_{n}^{a_{m}}, v_{s}^{i}\right)+\tilde{\phi}\left(v_{n}^{b_{m}}, v_{s}^{i}\right) \leq 1, \\
\tilde{\phi}\left(v_{n}^{a_{m}}, v_{s}^{i}\right)+\phi\left(v_{n}^{b_{m}}, v_{s}^{i}\right) \leq 1, \\
\forall i \in V_{s}, \forall n \in\left\{n \mid G_{n} \in \Phi\right\}, m=1,2, \ldots, L_{n}
\end{gathered}
$$

Fourth, it must be possible to re-map the off-peak traffic demand of all the virtual links belonging to the re-allocated virtual nodes. In this regard, first, we find the offpeak traffic demand between virtual nodes in every pair of modified substrate nodes to consider the re-allocation of virtual nodes. Supposing a pair of substrate nodes like $v_{s}^{i}$ and $v_{s}^{j}$, Table 4.1 summarizes the possible re-allocation combinations for the source virtual node $v_{n}^{a_{m}}$ and the sink virtual node $v_{n}^{b_{m}}$ of $l_{n}^{a_{m}, b_{m}}$ in these substrate nodes. 
Table 4.1: Re-allocation combinations for virtual nodes of $l_{n}^{a_{m}, b_{m}}$, and the required off-peak traffic to be rerouted

\begin{tabular}{|c|c|c|c|c|}
\hline$\phi\left(v_{n}^{a_{m}}, v_{s}^{i}\right)$ & $\tilde{\phi}\left(v_{n}^{a_{m}}, v_{s}^{i}\right)$ & $\phi\left(v_{n}^{b_{m}}, v_{s}^{j}\right)$ & $\tilde{\phi}\left(v_{n}^{b_{m}}, v_{s}^{j}\right)$ & Off-peak traffic to be rerouted \\
\hline 0 & 1 & 0 & 1 & $r_{n}^{m}$ \\
\hline 0 & 1 & 1 & 0 & $r_{n}^{m}$ \\
\hline 1 & 0 & 0 & 1 & $r_{n}^{m}$ \\
\hline 0 & 1 & 0 & 0 & 0 \\
\hline 1 & 0 & 1 & 0 & 0 \\
\hline 1 & 0 & 0 & 0 & 0 \\
\hline 0 & 0 & 0 & 1 & 0 \\
\hline 0 & 0 & 1 & 0 & 0 \\
\hline 0 & 0 & 0 & 0 & 0 \\
\hline
\end{tabular}

Table 4.1 determines the amount of off-peak traffic demand that needs to be reallocated due to reconfiguring the mapping of virtual links between virtual nodes on every pair of substrate nodes. For instance, the first row indicates the source virtual node of $l_{n}^{a_{m}, b_{m}}$ is re-allocated in $v_{s}^{i}$, and the sink virtual node of $l_{n}^{a_{m}, b_{m}}$ is re-allocated in $v_{s}^{j}$, so the off-peak traffic demand of $r_{n}^{m}$ needs to be re-mapped from the virtual node in $v_{s}^{i}$ to the virtual node in $v_{s}^{j}$. However, in the second row, the source virtual node of $l_{n}^{a_{m}, b_{m}}$ is re-allocated in $v_{s}^{i}$, while its sink virtual node is already mapped onto $v_{s}^{j}$, and it is not modified. Therefore, the off-peak traffic demand of $r_{n}^{m}$ needs to be re-mapped from virtual node in $v_{s}^{i}$ to the virtual node in $v_{s}^{j}$. The program derives the amount of off-peak traffic capacity that is required to be re-allocated between virtual nodes in any pair of physical nodes, for every virtual link.

Note that when both of the source and sink virtual nodes of a virtual link are re-allocated in other physical nodes (first row), the off-peak traffic demand should be re-mapped only between the re-allocated source and sink virtual nodes. However, according to Table 4.1, in this case, the off-peak traffic demand might also be remapped between the original virtual node and the re-allocated virtual nodes (second, or third row), which is not desirable.

In consequence, $d_{n}^{i, j}(m) r_{n}^{m}$ is the off-peak traffic demand of $l_{n}^{a_{m}, b_{m}}$ that needs to be re-allocated from the virtual node in $v_{s}^{i}$ to the virtual node in $v_{s}^{j}$. $d_{n}^{i, j}(m)$ can be derived as expressed in Equation 4.11. 
Hence, by specifying the amount of every off-peak traffic demand that needs remapping, the constraint in Equation 4.10 preserves one or multiple paths between the reconfigured source and sink virtual nodes. The constraint in Equation 4.10 and the objective function force the program to choose the path with the minimum number of active physical links. In the case there is no path between the modified virtual nodes, the program does not reconfigure the mapping of them.

$$
\begin{array}{r}
\sum_{\left\{j \mid(i, j) \in E_{s}\right\}} f_{n}^{\prime i, j}(m)-\sum_{\left\{j \mid(j, i) \in E_{s}\right\}} f_{n}^{\prime j, i}(m)=\sum_{j \in V_{s}} d_{n}^{i, j}(m) r_{n}^{m}-\sum_{j \in V_{s}} d_{n}^{j, i}(m) r_{n}^{m}, \\
\forall i \in V_{s}, \forall n \in\left\{n \mid G_{n} \in \Phi\right\}, m=1,2, \ldots, L_{n}
\end{array}
$$

where:

$$
\begin{array}{r}
d_{n}^{i, j}(m)=\left(1-\sum_{x \in V_{s}} \sum_{y \in V_{s}} \tilde{\phi}\left(v_{n}^{a_{m}}, v_{s}^{x}\right) \tilde{\phi}\left(v_{n}^{b_{m}}, v_{s}^{y}\right)\right)\left(\phi\left(v_{n}^{a_{m}}, v_{s}^{i}\right) \tilde{\phi}\left(v_{n}^{b_{m}}, v_{s}^{j}\right)\right. \\
\left.+\phi\left(v_{n}^{b_{m}}, v_{s}^{j}\right) \tilde{\phi}\left(v_{n}^{a_{m}}, v_{s}^{i}\right)\right)+\tilde{\phi}\left(v_{n}^{a_{m}}, v_{s}^{i}\right) \tilde{\phi}\left(v_{n}^{b_{m}}, v_{s}^{j}\right)
\end{array}
$$

As it is discussed, a substrate node $v_{s}^{i}$ might also forward the traffic of other virtual nodes and play the relay role. So, it is essential to re-map the virtual links that $v_{s}^{i}$ relays their traffic, before setting $v_{s}^{i}$ into the sleep mode. This is the link reconfiguration sub-problem. In this regard, if one or multiple relay substrate nodes for $l_{n}^{a_{m}, b_{m}}$ are set into the sleep mode, then Equation 4.12 sets the binary variable $\dot{\omega}_{n}^{m}$ to 1. Otherwise, $\dot{\omega}_{n}^{m}$ is set to 0 . In addition, if the source or sink virtual nodes of $l_{n}^{a_{m}, b_{m}}$, or both of them are re-allocated, then Equation 4.13 sets the binary variable $\ddot{\omega}_{n}^{m}$ to 1. Otherwise, $\ddot{\omega}_{n}^{m}$ is 0 . In this phase, we intend to re-map only the virtual links that one or multiple of their relay nodes over their allocated paths are set into the sleep mode, while their source and sink virtual nodes are not re-allocated. By finding the value of $\dot{\omega}_{n}^{m}$ and $\ddot{\omega}_{n}^{m}$ for every virtual link, $\dot{\omega}_{n}^{m}-\dot{\omega}_{n}^{m} \ddot{\omega}_{n}^{m}$ indicates whether the virtual link is needed to be re-mapped $\left(\dot{\omega}_{n}^{m}-\dot{\omega}_{n}^{m} \ddot{\omega}_{n}^{m}=1\right)$, or not $\left(\dot{\omega}_{n}^{m}-\dot{\omega}_{n}^{m} \ddot{\omega}_{n}^{m}=0\right)$. The constraint in Equation 4.14 re-maps the off-peak traffic demand of virtual links that one or multiple of their relay substrate nodes are set into the sleep mode, and their source and sink virtual nodes are not re-allocated. Note that the constraint in Equation 4.10 re-maps a virtual link, as a part of node reconfiguration sub-problem, if its source or sink virtual node, or both of them are re-allocated in other physical nodes. 
Therefore, the program does not consider re-mapping of such a virtual link, again.

$$
\begin{aligned}
& \dot{\omega}_{n}^{m} \leq \sum_{i \in V_{s}} \varrho_{n}^{\prime m}\left(v_{s}^{i}\right)\left(1-\alpha\left(v_{s}^{i}\right)\right) \leq B_{2} \dot{\omega}_{n}^{m}, \\
& \ddot{\omega}_{n}^{m} \leq \sum_{i \in V_{s}}\left(\tilde{\phi}\left(v_{n}^{a_{m}}, v_{s}^{i}\right)+\tilde{\phi}\left(v_{n}^{b_{m}}, v_{s}^{i}\right)\right) \leq B_{3} \ddot{\omega}_{n}^{m}, \\
& \forall n \in\left\{n \mid G_{n} \in \Phi\right\}, m=1,2, \ldots, L_{n} \\
& \sum_{\left\{j \mid(i, j) \in E_{s}\right\}} f_{n}^{i, j}(m)-\sum_{\left\{j \mid(j, i) \in E_{s}\right\}} f_{n}^{j, i}(m)=\left\{\begin{array}{ll}
\left(\dot{\omega}_{n}^{m}-\dot{\omega}_{n}^{m} \ddot{\omega}_{n}^{m}\right) \dot{r}_{n}^{m} & \text { if } i=a_{m} \\
\left(\dot{\omega}_{n}^{m} \ddot{\omega}_{n}^{m}-\dot{\omega}_{n}^{m}\right) \dot{r}_{n}^{m} & \text { if } i=b_{m} \\
0 & \text { otherwise }
\end{array},\right. \\
& \forall i \in V_{s}, \forall n \in\left\{n \mid G_{n} \in \Phi\right\}, m=1,2, \ldots, L_{n}
\end{aligned}
$$

Re-allocating virtual links by either the constraint in Equation 4.10 or the constraint in Equation 4.14, also requires satisfying some other constraints. First, the program ensures in the constraint in Equation 4.15 that no rerouted traffic passes through the sleeping physical nodes. The constraint in Equation 4.15 ensures total outgoing re-allocated off-peak traffic capacity $\sum_{\left\{j \mid(i, j) \in E_{s}\right\}} \sum_{\left\{n \mid G_{n} \in \Phi\right\}} \sum_{m=1}^{L_{n}}\left(f_{n}^{i, j}(m)+f_{n}^{\prime, j}(m)\right)$, and the total incoming re-allocated off-peak traffic capacity $\sum_{\left\{j \mid(j, i) \in E_{s}\right\}} \sum_{\left\{n \mid G_{n} \in \Phi\right\}} \sum_{m=1}^{L_{n}}\left(f_{n}^{j, i}(m)+f_{n}^{\prime j, i}(m)\right)$, in a sleeping physical node $\left(\alpha\left(v_{s}^{i}\right)=0\right)$ is 0 .

$$
\begin{aligned}
& \sum_{\left\{j \mid(i, j) \in E_{s}\right\}} \sum_{\left\{n \mid G_{n} \in \Phi\right\}} \sum_{m=1}^{L_{n}}\left(f_{n}^{i, j}(m)+f_{n}^{\prime i, j}(m)\right) \\
& +\sum_{\left\{j \mid(j, i) \in E_{s}\right\}} \sum_{\left\{n \mid G_{n} \in \Phi\right\}} \sum_{m=1}^{L_{n}}\left(f_{n}^{j, i}(m)+f_{n}^{\prime j, i}(m)\right) \leq B_{4} \alpha\left(v_{s}^{i}\right),
\end{aligned}
$$

$\forall i \in V_{s}$

Second, the total embedded off-peak traffic capacity in every substrate link must be equal or less than its physical bandwidth capacity, as indicated in the constraint in Equation 4.16. In this program, three types of traffic capacities form the total embedded off-peak traffic capacity in a substrate link $l_{s}^{i, j}$. The first type is the off-peak traffic 
capacity of the virtual links that are not re-allocated through the reconfiguration process. In this regard, none of the relay substrate nodes in the already allocated paths for the virtual link is set into the sleep mode, and therefore $1-\dot{\omega}_{n}^{m}=1$. Moreover, the source and sink virtual nodes of the virtual link are not re-allocated during the reconfiguration process, so $1-\ddot{\omega}_{n}^{m}=1$. Nonetheless, if the virtual link is re-allocated, then this off-peak traffic is not considered. The second type is the re-allocated offpeak traffic capacity $f_{n}^{\prime i, j}(m)$ to a virtual link in the substrate link, as the result of node reconfiguration sub-problem. However, the third type is the re-allocated offpeak traffic capacity $f_{n}^{i, j}(m)$ to a virtual link in the substrate link, as the result of link reconfiguration sub-problem. Equation 4.17 defines the total traffic in a substrate link.

$$
r\left(l_{s}^{i, j}\right) \leq C_{b}\left(l_{s}^{i, j}\right), \quad \forall(i, j) \in E_{s}
$$

where:

$$
r\left(l_{s}^{i, j}\right)=\sum_{\left\{n \mid G_{n} \in \Phi\right\}} \sum_{m=1}^{L_{n}}\left(\left(1-\dot{\omega}_{n}^{m}\right)\left(1-\ddot{\omega}_{n}^{m}\right) \dot{r}_{n}^{i, j}(m)+f_{n}^{\prime i, j}(m)+f_{n}^{i, j}(m)\right)
$$

Third, the total embedded off-peak traffic capacity in every substrate node must be equal or less than its physical bandwidth capacity, as indicated in Equation 4.18. Note that the total traffic in a substrate node is the total traffic in its incoming physical links plus the total traffic in its outgoing physical links. Equation 4.19 defines the total traffic in a substrate node.

$$
r\left(v_{s}^{i}\right) \leq C_{b}\left(v_{s}^{i}\right), \quad \forall i \in V_{s}
$$

where:

$$
r\left(v_{s}^{i}\right)=\sum_{\left\{j \mid(i, j) \in E_{s}\right\}} r\left(l_{s}^{i, j}\right)+\sum_{\left\{j \mid(j, i) \in E_{s}\right\}} r\left(l_{s}^{j, i}\right)
$$

Furthermore, we do not re-allocate the allocated virtual nodes in high stressed substrate nodes, and the virtual links that all the physical nodes along their allocated paths are high stressed. The constraint in Equation 4.20 determines a physical node $v_{s}^{i}$ with $\tilde{s_{1}}\left(v_{s}^{i}\right) \geq \mathcal{T}$, must stay active $\alpha\left(v_{s}^{i}\right)=1$. Consequently, Equation 4.6 does not let the program to re-allocate any allocated virtual node in the physical node $v_{s}^{i}$. Besides, Equation 4.12, and Equation 4.13 ensure a virtual link that all the physical 
nodes along their allocated paths are high stressed is not re-allocated.

$$
\forall i \in\left\{i \mid i \in V_{s}, \tilde{s_{1}}\left(v_{s}^{i}\right) \geq \mathcal{T}\right\}: \quad \alpha\left(v_{s}^{i}\right)=1
$$

The constraint in Equation 4.21 makes the program linear by setting the auxiliary binary variable $\alpha\left(l_{s}^{i, j}\right)$ to 1 , if there is any traffic in $l_{s}^{i, j}$. Otherwise, $\alpha\left(l_{s}^{i, j}\right)$ is 0 .

$$
r\left(l_{s}^{i, j}\right) \leq B_{5} \alpha\left(l_{s}^{i, j}\right), \quad \forall(i, j) \in E_{s}
$$

The variables also must be in the following bounds:

$$
\begin{gathered}
f_{n}^{i, j}(m) \geq 0, f_{n}^{\prime i, j}(m) \geq 0, \quad \forall(i, j) \in E_{s}, \forall n \in\left\{n \mid G_{n} \in \Phi\right\}, m=1,2, \ldots, L_{n} \\
\alpha\left(l_{s}^{i, j}\right) \in\{0,1\}, \quad \forall(i, j) \in E_{s} \\
\alpha\left(v_{s}^{i}\right) \in\{0,1\}, \quad \forall i \in V_{s} \\
\tilde{\phi}\left(v_{n}^{k}, v_{s}^{i}\right) \in\{0,1\}, \quad \forall i \in V_{s}, \forall n \in\left\{n \mid G_{n} \in \Phi\right\}, \forall k \in V_{n} \\
\dot{\omega}_{n}^{m} \in\{0,1\}, \ddot{\omega}_{n}^{m} \in\{0,1\}, \quad \forall n \in\left\{n \mid G_{n} \in \Phi\right\}, m=1,2, \ldots, L_{n}
\end{gathered}
$$

There are some constraints in the program that include a product of binary variables. This issue makes the program nonlinear. However, a product of two binary variables can be replaced by one new binary variable, on which a number of constraints is imposed to the program [90], and therefore the program remains linear. The extension to the products of more binary variables is straightforward. Considering the binary variables $x_{1}$ and $x_{2}$, their product $x_{1} x_{2}$ could be replaced by a new binary variable $y$, while the following constraints force $y$ to take the value of $x_{1} x_{2}$.

$$
\begin{gathered}
y \leq x_{1} \\
y \leq x_{2} \\
y \geq x_{1}+x_{2}-1 \\
y \in\{0,1\}
\end{gathered}
$$

As it is described, the formulated MILP for ONL-LNLs-F includes two subproblems. The link reconfiguration sub-problem could be reduced to the problem 
discussed in [83] that is a simple two-commodity integer flow problem. It is proven in [83] that this simple two-commodity integer flow problem is $\mathcal{N} \mathcal{P}$-hard. Hence, our formulated MILP for off-peak node and link energy optimization by local node and link reconfiguration is $\mathcal{N} \mathcal{P}$-hard.

\section{Off-peak Node and Link Energy Optimization by Local Link Reconfigu- ration}

We define a different stress rate for an intermediate substrate node in this section. Accordingly, a solution is proposed to optimize total energy consumption of the intermediate substrate nodes and substrate links during the off-peak time. We do not re-map a virtual node in this problem. This method might set the less stressed intermediate substrate nodes and their respective substrate links into the sleep mode for the off-peak time. We re-map a virtual link if and only if we sleep at least one intermediate substrate node over its embedded path. The programs also concern with a VNE's link energy consumption, by setting the maximum number of physical links into the sleep mode during the re-allocation of virtual links. An MILP for splittable traffic and a BILP for non-splittable traffic are formulated for this strategy.

The stress rate $\tilde{s_{2}}\left(v_{s}^{i}\right)$ of a physical node $v_{s}^{i}$ in this problem, indicates the intensity of involved VNs and the total off-peak traffic demand in the substrate node. The definition of a physical node's stress rate in this problem is different to the definition of a physical node's stress rate in the previous problem, because we do not reconfigure virtual nodes here. A VN is involved in a substrate node $v_{s}^{i}$, if at least one of its virtual nodes is mapped onto $v_{s}^{i}$, or at least one of its mapped virtual links passes through $v_{s}^{i}$. Assume $\eta\left(v_{s}^{i}\right)$ as the number of virtual networks involved in a physical node $v_{s}^{i}$, the following equation defines $\tilde{s_{2}}\left(v_{s}^{i}\right)$.

$$
\begin{array}{r}
\tilde{s_{2}}\left(v_{s}^{i}\right)=\frac{\eta\left(v_{s}^{i}\right)}{|\Phi|}\left(\frac{\sum_{\left\{j \mid(i, j) \in E_{s}\right\}} \sum_{\left\{n \mid G_{n} \in \Phi\right\}} \sum_{m=1}^{L_{n}} \dot{r}_{n}^{i, j}(m)}{C_{b}\left(v_{s}^{i}\right)}\right. \\
\left.+\frac{\sum_{\left\{j \mid(j, i) \in E_{s}\right\}} \sum_{\left\{n \mid G_{n} \in \Phi\right\}} \sum_{m=1}^{L_{n}} r_{n}^{j, i}(m)}{C_{b}\left(v_{s}^{i}\right)}\right)
\end{array}
$$

$\tilde{s_{2}}\left(v_{s}^{i}\right)$ considers two parameters. The first parameter is the fraction of the number of involved VNs in a node, over the total number of active VNs. This parameter denotes the intensity of involved $\mathrm{VNs}$ in a $v_{s}^{i}$. However, the second parameter considers 
the off-peak traffic demand by finding the fraction of total off-peak traffic passes a substrate node, over the bandwidth capacity of the node. Consequently, in this problem, a higher physical node's stress rate means a larger number of VNs is involved in the node, while the substrate node is more utilized in terms of off-peak traffic. In this regard, we do not set an intermediate physical node with $\tilde{s_{2}}\left(v_{s}^{i}\right) \geq \mathcal{T}$ into the sleep mode. Virtual links that the stress rate of all the physical nodes along their allocated paths are equal or greater than $\mathcal{T}$ are also not re-mapped. These actions help to decrease the traffic disruptions due to the reconfiguration. Note that the threshold value of $\mathcal{T}$ is adjustable. The providers could adjust the value of $\mathcal{T}$, and therefore manage the level of the reconfiguration. Decreasing the value of $\tilde{s_{2}}$ threshold degrades the amount of energy the programs could save and reduces the possible traffic interruptions, because a smaller number of physical nodes and links will be considered for energy-saving. The impact of different values for the stress rate threshold on the energy-saving ability of the solutions is discussed in Section 4.5.

The traffic might be splittable or non-splittable. In the splittable case, a traffic demand of each virtual link could be carried by one or multiple paths in a substrate network. However, if the traffic is non-splittable, then each virtual link's traffic demand may be required to follow the same path through the network, rather than be divided among multiple paths. It is expected to save higher amounts of energy when traffic is splittable, because we are more flexible in terms of finding alternative paths. This is a major restriction that has an important impact on the solution's methodology. In this regard, we formulate off-peak node and link energy optimization by local link reconfiguration for both splittable and non-splittable traffic.

Splittable Traffic (ONL-LLs-F) The defined local link reconfiguration problem, in the case of splittable traffic, can be formulated as an MILP, in the category of multi-commodity flow problems. In the context of this problem, every virtual link is a commodity. The off-peak node and link energy optimization by local link reconfiguration that is formulated based on Fixed power model, when traffic is splittable, is called ONL-LLs-F.

Similar to the previous problem, the substrate network topology is specified. The physical bandwidth capacity of every substrate node and link is known. Allocated virtual networks are also identified. In this problem, we have $L_{n}$ commodities for the $n$th virtual network. For each virtual link $l_{n}^{a_{m}, b_{m}}$, as a commodity, we are given a 
scalar $r_{n}^{m}$ that refers to the virtual link's off-peak traffic demand. $r_{n}^{i, j}(m)$ for every virtual link in each physical link is also known. Besides, $\varrho_{n}^{m}\left(v_{s}^{i}\right)$ indicates whether the allocated path for $l_{n}^{a_{m}, b_{m}}$ includes $v_{s}^{i}$, or not. $\varrho_{n}^{m}\left(v_{s}^{i}\right)$ is 1 , if the allocated path for $l_{n}^{a_{m}, b_{m}}$ contains $v_{s}^{i}$. Otherwise, $\varrho_{n}^{m}\left(v_{s}^{i}\right)$ is 0 .

Therefore, ONL-LLs-F is formulated as an MILP as follows:

Optimization Variables:

- $\alpha\left(v_{s}^{i}\right)$ is a binary variable. It denotes the status of $v_{s}^{i} \cdot \alpha\left(v_{s}^{i}\right)$ is 1 in the case $v_{s}^{i}$ is active, otherwise it is 0 .

- $\alpha\left(l_{s}^{i, j}\right)$ is a binary variable. It refers to the status of $l_{s}^{i, j} \cdot \alpha\left(l_{s}^{i, j}\right)$ is 1 in the case $l_{s}^{i, j}$ is active, otherwise it is 0.

- $\omega_{n}^{m}$ is a binary variable. It is 1 in the case at least one physical node over the allocated path for $l_{n}^{a_{m}, b_{m}}$ is set into the sleep mode. Otherwise, it is 0 .

- $f_{n}^{i, j}(m)$ is a real-valued variable. It denotes the re-allocated off-peak traffic capacity to the $m$ th virtual link of the $n$th virtual network, in $l_{s}^{i, j}$.

Objective Function: This program aims to minimize the total energy consumption by physical intermediate nodes and physical links during the off-peak time, according to Fixed power model. Equation 4.32, and the constraint in Equation 4.34 that allows only intermediate substrate nodes to be set into the sleep mode, preserve this objective.

$$
\text { Minimize }\left\{\sum_{i \in V_{s}} \alpha\left(v_{s}^{i}\right) \tilde{p}^{m}\left(v_{s}^{i}\right)+\sum_{(i, j) \in E_{s}} \alpha\left(l_{s}^{i, j}\right) \tilde{p}^{m}\left(l_{s}^{i, j}\right)\right\}
$$

Constraints: If the program puts an intermediate physical node into the sleep mode, then it needs to suggest one or more alternative paths for every virtual link that was passing the substrate node. In this regard, the constraint in Equation 4.33 sets the binary variable $\omega_{n}^{m}$ to 1 , if at least one physical node $v_{s}^{i}$, that is on the allocated path for $l_{n}^{a_{m}, b_{m}}\left(\varrho_{n}^{m}\left(v_{s}^{i}\right)=1\right)$, is set into the sleep mode $\left(\alpha\left(v_{s}^{i}\right)=0\right)$. Otherwise, $\omega_{n}^{m}$ is 0 . The program derives the $\omega_{n}^{m}$ for every virtual link. $\omega_{n}^{m}$ determines whether $l_{n}^{a_{m}, b_{m}}$ is required to be re-mapped, or not. Thus, the constraint in Equation 4.34 preserves at least one alternative path from the source virtual node $v_{n}^{a_{m}}$ to the sink virtual node $v_{n}^{b_{m}}$ of $l_{n}^{a_{m}, b_{m}}$, if $\omega_{n}^{m}$ is 1 . In the case $\omega_{n}^{m}$ is $0, l_{n}^{a_{m}, b_{m}}$ is not re-mapped. Note that the constraint in Equation 4.34 does not allow a physical node that at least one virtual 
node is allocated in it, to be set into the sleep mode. The program also considers a VNE's link energy consumption by re-allocating virtual links in the substrate paths with the minimum number of physical links. Nevertheless, it is also necessary to satisfy some other constraints when re-allocating a virtual link. Note that $B_{1}, B_{2}$, and $B_{3}$ are integer numbers, while they must be large enough to be greater than the left hand side of their respective equations.

$$
\begin{array}{r}
\omega_{n}^{m} \leq \sum_{i \in V_{s}} \varrho_{n}^{m}\left(v_{s}^{i}\right)\left(1-\alpha\left(v_{s}^{i}\right)\right) \leq B_{1} \omega_{n}^{m}, \quad \forall n \in\left\{n \mid G_{n} \in \Phi\right\}, m=1,2, \ldots, L_{n} \\
\sum_{\left\{j \mid(i, j) \in E_{s}\right\}} f_{n}^{i, j}(m)-\sum_{\left\{j \mid(j, i) \in E_{s}\right\}} f_{n}^{j, i}(m)= \begin{cases}\omega_{n}^{m} r_{n}^{m} & \text { if } i=a_{m} \\
-\omega_{n}^{m} r_{n}^{m} & \text { if } i=b_{m}, \\
0 \quad & \text { otherwise }\end{cases} \\
\forall i \in V_{s}, \forall n \in\left\{n \mid G_{n} \in \Phi\right\}, m=1,2, \ldots, L_{n},
\end{array}
$$

First, it is not feasible that the re-allocated off-peak traffic passes through the physical nodes in the sleep mode. So, the constraint in Equation 4.35 ensures the total incoming and outgoing re-allocated traffic passing through a physical node in the sleep mode $\left(\alpha\left(v_{s}^{i}\right)=0\right)$ is 0 .

$$
\sum_{\left\{j \mid(i, j) \in E_{s}\right\}} \sum_{\left\{n \mid G_{n} \in \Phi\right\}} \sum_{m=1}^{L_{n}} f_{n}^{i, j}(m)+\sum_{\left\{j \mid(j, i) \in E_{s}\right\}} \sum_{\left\{n \mid G_{n} \in \Phi\right\}} \sum_{m=1}^{L_{n}} f_{n}^{j, i}(m) \leq B_{2} \alpha\left(v_{s}^{i}\right), \quad \forall i \in V_{s}
$$

Second, the total embedded off-peak traffic capacity in every substrate link must be equal or less than its physical bandwidth capacity. Equation 4.36 preserves this constraint. In this problem, two types of traffic capacities form the total embedded off-peak traffic capacity in a substrate link $l_{s}^{i, j}$. The first type is the embedded offpeak traffic capacity to the virtual links that are not re-allocated by the program. $1-\omega_{n}^{m}$ determines whether an $l_{n}^{a_{m}, b_{m}}$ is re-allocated or not. If the program re-maps $l_{n}^{a_{m}, b_{m}}$, then $\omega_{n}^{m}$ is 1 . Therefore, $1-\omega_{n}^{m}$ is 0 , which means $l_{n}^{a_{m}, b_{m}}$ is re-allocated, and so its off-peak traffic capacity does not exist in its original path. On the other hand, if $l_{n}^{a_{m}, b_{m}}$ is not re-allocated, then $1-\omega_{n}^{m}$ is 1 , and its off-peak traffic capacity is counted. 
The second type is the re-allocated off-peak traffic capacity $f_{n}^{i, j}(m)$ to virtual links in the substrate link. Consequently, $\sum_{\left\{n \mid G_{n} \in \Phi\right\}} \sum_{m=1}^{L_{n}}\left(\left(1-\omega_{n}^{m}\right) r_{n}^{i, j}(m)+f_{n}^{i, j}(m)\right)$ is the total embedded off-peak traffic capacity in $l_{s}^{i, j}$.

$$
r\left(l_{s}^{i, j}\right) \leq C_{b}\left(l_{s}^{i, j}\right), \quad \forall(i, j) \in E_{s}
$$

where:

$$
r\left(l_{s}^{i, j}\right)=\sum_{\left\{n \mid G_{n} \in \Phi\right\}} \sum_{m=1}^{L_{n}}\left(\left(1-\omega_{n}^{m}\right) r_{n}^{i, j}(m)+f_{n}^{i, j}(m)\right)
$$

Third, the total embedded off-peak traffic capacity in a substrate node must be equal or less than its physical bandwidth capacity $C_{b}\left(v_{s}^{i}\right)$, as expressed in 4.38. The total traffic in a substrate node is the total traffic in its incoming physical links plus the total off-peak traffic in its outgoing physical links. Equation 4.39 defines the total traffic in a substrate node.

$$
r\left(v_{s}^{i}\right) \leq C_{b}\left(v_{s}^{i}\right), \quad \forall i \in V_{s}
$$

where:

$$
r\left(v_{s}^{i}\right)=\sum_{\left\{j \mid(i, j) \in E_{s}\right\}} r\left(l_{s}^{i, j}\right)+\sum_{\left\{j \mid(j, i) \in E_{s}\right\}} r\left(l_{s}^{j, i}\right)
$$

As it is discussed, we do not sleep the high stressed physical nodes. The constraint in Equation 4.40 ensures a substrate node with $\tilde{s_{2}}\left(v_{s}^{i}\right) \geq \mathcal{T}$ remains active. Besides, if all the physical nodes along the allocated path for a virtual link are high stressed, then the constraint in Equation 4.33 ensures the virtual link is not re-allocated.

$$
\alpha\left(v_{s}^{i}\right)=1, \quad \forall i \in\left\{i \mid i \in V_{s}, \tilde{s_{2}}\left(v_{s}^{i}\right) \geq \mathcal{T}\right\}
$$

The constraint in Equation 4.41 makes the program linear, similar to the first program.

$$
r\left(l_{s}^{i, j}\right) \leq B_{3} \alpha\left(l_{s}^{i, j}\right), \quad \forall(i, j) \in E_{s}
$$

The variables also must hold the following bounds:

$$
\begin{gathered}
f_{n}^{i, j}(m) \geq 0, \quad \forall(i, j) \in E_{s}, \forall n \in\left\{n \mid G_{n} \in \Phi\right\}, m=1,2, \ldots, L_{n} \\
\alpha\left(l_{s}^{i, j}\right) \in\{0,1\}, \quad \forall(i, j) \in E_{s}
\end{gathered}
$$




$$
\begin{gathered}
\alpha\left(v_{s}^{i}\right) \in\{0,1\}, \quad \forall i \in V_{s} \\
\omega_{n}^{m} \in\{0,1\}, \quad \forall n \in\left\{n \mid G_{n} \in \Phi\right\}, m=1,2, \ldots, L_{n}
\end{gathered}
$$

Non-Splittable Traffic (ONL-LLns-F) The traffic might be non-splittable, that is each traffic demand has to be carried on the same path. The defined local link reconfiguration problem, in the case of non-splittable traffic, can be formulated as a BILP in the category of multi-commodity flow problems. Similar to ONL-LLs-F, each virtual link is a commodity in the context of this problem. A virtual link as a commodity generates traffic that flows through its allocated path. Different from when traffic is splittable, traffic of every re-mapped virtual link must be carried in a single substrate path. The off-peak node and link energy optimization by local link reconfiguration that is formulated based on Fixed power model, while traffic is non-splittable, is called ONL-LLns-F. The input of this problem is the same as the input for the formulated MILP for ONL-LLs-F.

ONL-LLns-F is formulated as a BILP as follows:

Optimization Variables:

- $\alpha\left(v_{s}^{i}\right)$ is a binary variable. $\alpha\left(v_{s}^{i}\right)$ denotes the status of $v_{s}^{i} \cdot \alpha\left(v_{s}^{i}\right)$ is 1 in the case $v_{s}^{i}$ is active, otherwise it is 0 .

- $\alpha\left(l_{s}^{i, j}\right)$ is a binary variable. $\alpha\left(l_{s}^{i, j}\right)$ refers to the status of $l_{s}^{i, j} \cdot \alpha\left(l_{s}^{i, j}\right)$ is 1 in the case $l_{s}^{i, j}$ is active, otherwise it is 0 .

- $\omega_{n}^{m}$ is a binary variable. $\omega_{n}^{m}$ is 1 in the case at least one physical node over the allocated path for $l_{n}^{a_{m}, b_{m}}$, is set into the sleep mode. Otherwise, $\omega_{n}^{m}$ is 0 .

- $z_{n}^{i, j}(m)$ is a binary variable. $z_{n}^{i, j}(m)$ is 1 in the case the re-mapped path to $l_{n}^{a_{m}, b_{m}}$ includes $l_{s}^{i, j}$. Otherwise, $z_{n}^{i, j}(m)$ is 0 .

Objective Function: The same objective as Equation 4.32.

Constraints: The constraints in Equations 4.33, 4.36, 4.38, 4.40, 4.41 and the followings: This program works almost the same as the formulated program for ONL-LLs-F. However, different from the developed program for ONL-LLs-F, and since traffic is non-splittable, the BILP must find only a single alternative path for any re-allocated virtual link. In this regard, if the program needs to re-map an $l_{n}^{a_{m}, b_{m}}$, then $\omega_{n}^{m}$ is 1 . Therefore, the constraint in Equation 4.46 requires routing a single unit of data from $v_{n}^{a_{m}}$ to $v_{n}^{b_{m}}$. As the variable $z_{n}^{i, j}(m)$ is binary, the unit of data could not be splitted. 
Besides, Equation 4.47 limits the program routing, so the maximum number of incoming and outgoing flows for any node is two, to prevent from having a loop. Thus, the driven route will be a single path from $v_{n}^{a_{m}}$ to $v_{n}^{b_{m}}$, which will be used as the replaced path for $l_{n}^{a_{m}, b_{m}}$.

$$
\begin{gathered}
\sum_{\left\{j \mid(i, j) \in E_{s}\right\}} z_{n}^{i, j}(m)-\sum_{\left\{j \mid(j, i) \in E_{s}\right\}} z_{n}^{j, i}(m)= \begin{cases}\omega_{n}^{m} \quad \text { ifi }=a_{m} \\
-\omega_{n}^{m} \quad \text { ifi } i=b_{m} \\
0 \quad \text { otherwise }\end{cases} \\
\forall i \in V_{s}, \forall n \in\left\{n \mid G_{n} \in \Phi\right\}, m=1,2, \ldots, L_{n}
\end{gathered}
$$

The constraint in Equation 4.48 ensures any rerouted traffic flow does not pass through the nodes in the sleep mode.

$$
\begin{array}{r}
\sum_{\left\{j \mid(i, j) \in E_{s}\right\}} \sum_{\left\{n \mid G_{n} \in \Phi\right\}} \sum_{m=1}^{L_{n}} z_{n}^{i, j}(m)+\sum_{\left\{j \mid(j, i) \in E_{s}\right\}} \sum_{\left\{n \mid G_{n} \in \Phi\right\}} \sum_{m=1}^{L_{n}} z_{n}^{j, i}(m) \leq B_{2} \alpha\left(v_{s}^{i}\right) \\
\forall i \in V_{s}
\end{array}
$$

Note that the total embedded off-peak traffic capacity in any physical link is calculated similar to the previous program, but, here, $z_{n}^{i, j}(m) r_{n}^{m}$ determines the re-allocated offpeak traffic capacity to a virtual link $l_{n}^{a_{m}, b_{m}}$ in an $l_{s}^{i, j}$. If the off-peak traffic demand $r_{n}^{m}$ of $l_{n}^{a_{m}, b_{m}}$ is rerouted through $l_{s}^{i, j}$, then $z_{n}^{i, j}(m) r_{n}^{m}$ is equal to $r_{n}^{m}$. Otherwise, this amount is 0. This is reflected in Equation 4.49.

$$
r\left(l_{s}^{i, j}\right)=\sum_{\left\{n \mid G_{n} \in \Phi\right\}} \sum_{m=1}^{L_{n}}\left(\left(1-\omega_{n}^{m}\right) \dot{r}_{n}^{i, j}(m)+z_{n}^{i, j}(m) r_{n}^{m}\right)
$$

The variables must hold in the bounds in Equations 4.43, 4.44, 4.45 and the following:

$$
z_{n}^{i, j}(m) \in\{0,1\}, \forall(i, j) \in E_{s}, \forall n \in\left\{n \mid G_{n} \in \Phi\right\}, m=1,2, \ldots, L_{n}
$$


The rest of the program works similar to the formulated program for ONL-LLs-F.

Both of the formulated integer linear programs for ONL-LLs-F and ONL-LLnsF can be reduced to the problem discussed in [83] that is a simple two-commodity integer flow problem. It is proven in [83] that this simple two-commodity integer flow problem is $\mathcal{N} \mathcal{P}$-hard. Hence, the formulated ILPs for off-peak node and link energy optimization by local link reconfiguration, either in the case of splittable traffic demands, or non-splittable traffic demands, are $\mathcal{N} \mathcal{P}$-hard.

\subsubsection{Programs Based on Semi-Proportional Power Model}

The previous section developed energy-saving programs for a VNE's nodes and links, conforming to Fixed link power model. According to Fixed link power model, an active physical node and link consumes a constant amount of power, regardless of its traffic load. Therefore, the formulated programs reduce a VNE's total node and link energy consumption by setting physical nodes and links into the sleep mode.

Nevertheless, considering a constant amount of power consumption for an active physical node or link is not efficient. As it is discussed in Section 4.2, SemiProportional link power model defines a traffic-adaptive power model for a physical node or link. Based on this power model, a large portion of consumed power by a physical node or link is for keeping the device operational. Nonetheless, different from Fixed link power model, traffic load in the network element also changes its energy consumption. In this regard, it may be possible to reduce the node or link energy consumption by either setting the device into the sleep mode, or by rerouting its traffic load to the other active part of the network. However, we could save a larger amount of energy by sleeping the device, in comparison to rerouting its traffic load.

Consequently, the energy-saving solutions for Semi-Proportional power model are different from the Fixed link power model solutions. The defined programs in the previous section are required to be modified in order to optimize the energy based on Semi-Proportional power model. In this regard, it is essential to modify the objective functions defined in each of the formulated program to minimize the node and link energy consumption based on Semi-Proportional power model. The power consumption of a single physical node according to Semi-Proportional power model is defined in Equation 2.46. In addition, the power consumption of a single physical link based on this model is also defined in Equation 2.59. Accordingly, it is needed to replace 
the stated objective functions in Equation 4.5, and Equation 4.32 by the new objective function defined in Equation 4.51, that is formulated based on Semi-Proportional power model.

$$
\begin{aligned}
\operatorname{Minimize}\left\{\sum_{i \in V_{s}}\left(\alpha\left(v_{s}^{i}\right) \tilde{p}^{b}\left(v_{s}^{i}\right)+\frac{r\left(v_{s}^{i}\right)}{C_{b}\left(v_{s}^{i}\right)}\left(\tilde{p}^{m}\left(v_{s}^{i}\right)-\tilde{p}^{b}\left(v_{s}^{i}\right)\right)\right)\right. \\
\left.\quad+\sum_{(i, j) \in E_{s}}\left(\alpha\left(l_{s}^{i, j}\right) \tilde{p}^{b}\left(l_{s}^{i, j}\right)+\frac{r\left(l_{s}^{i, j}\right)}{C_{b}\left(l_{s}^{i, j}\right)}\left(\tilde{p}^{m}\left(l_{s}^{i, j}\right)-\tilde{p}^{b}\left(l_{s}^{i, j}\right)\right)\right)\right\}
\end{aligned}
$$

By modifying the objective functions of the formulated programs, and keeping the same constraints and bounds, the programs optimize the node and link energy consumption of a VNE, during the off-peak period, based on Semi-Proportional power model.

Note that off-peak node and link energy optimization by local node and link reconfiguration, that is formulated based on Semi-Proportional power model, when traffic is splittable, is called ONL-LNLs-SP. Besides, the off-peak node and link energy optimization by local link reconfiguration problem that is defined according to SemiProportional power model is called ONL-LLs-SP in the case of splittable traffic, and ONL-LLns-SP in the case of non-splittable traffic.

\subsection{The Heuristic Algorithm}

The discussed BILP for ONL-LLns-F in Section 4.3.2 adjusts some of the already allocated virtual links in order to reduce the total node and link energy consumption in VNE, during the off-peak time, while traffic is non-splittable. Nevertheless, the formulated BILP for ONL-LLns-F is $\mathcal{N} \mathcal{P}$-hard, and therefore the optimization solution is not scalable in the case of large network sizes, due to the long executing time. Hence, in this section, we propose an energy-saving heuristic for ONL-LLns-F. The pseudo code of the heuristic is shown in Algorithm 2.

We know from Semi-Proportional power models that a very large part of the power consumption of a physical node or link is for its base power consumption rather than the traffic related power consumption. Therefore, targeting only the base power consumption of a device still provides an effective energy-saving method. Hence, to 
simplify the heuristic and decrease its time complexity, it is defined based on Fixed power model. This means the heuristic does not save energy by rerouting the traffic.

Accordingly, the physical nodes consume more energy than physical links. In this regard, the algorithm first calculates some metrics and then sorts the intermediate physical nodes, according to their stress rate, in order to check the node removal possibility. Afterwards, it attempts to find an alternative path, with the minimum number of physical links, for the off-peak traffic of every virtual link that was passing the sleeping physical nodes.

The stress rate $\tilde{s_{2}}\left(v_{s}^{i}\right)$ for a substrate node $v_{s}^{i}$, could be found in a similar way that is calculated in Equation 4.31. $\tilde{s_{2}}\left(v_{s}^{i}\right)$ defines the intensity of involved VNs and the total off-peak traffic in a substrate node $v_{s}^{i}$. This helps us in sorting and decisionmaking matters in the following phases.

A virtual link might get mapped onto a single physical link or multiple physical links. In either cases, the requested bandwidth will be allocated in the physical nodes and links. Because the virtual networks' traffic rates are decreased during the off-peak time, the whole allocated virtual link capacity is not used. During this period, the off-peak demand is the reserved capacity in a substrate node or link, and the remained bandwidth capacity could be shared. The unused off-peak bandwidth capacity in an $l_{s}^{i, j}$ is represented by $\breve{C}_{b}\left(l_{s}^{i, j}\right) . \breve{C}_{b}\left(l_{s}^{i, j}\right)$ is equal to the physical link's bandwidth capacity $C_{b}\left(l_{s}^{i, j}\right)$ subtracted by the total off-peak traffic demand in $l_{s}^{i, j}$. Equation 4.52 defines $\breve{C}_{b}\left(l_{s}^{i, j}\right)$. Besides, as it is stated in Equation 4.39, the total traffic in a physical node $v_{s}^{i}$ is equal to the total outgoing and incoming traffic of the node. Accordingly, the unused off-peak bandwidth capacity $\breve{C}_{b}\left(v_{s}^{i}\right)$ of a $v_{s}^{i}$ could be defined in Equation 4.53.

$$
\begin{gathered}
\breve{C}_{b}\left(l_{s}^{i, j}\right)=C_{b}\left(l_{s}^{i, j}\right)-\sum_{\left\{n \mid G_{n} \in \Phi\right\}} \sum_{m=1}^{L_{n}} \dot{r}_{n}^{i, j}(m) \\
\breve{C}_{b}\left(v_{s}^{i}\right)=C_{b}\left(v_{s}^{i}\right)-\left(\sum_{\left\{j \mid(i, j) \in E_{s}\right\}} \sum_{\left\{n \mid G_{n} \in \Phi\right\}} \sum_{m=1}^{L_{n}} \dot{r}_{n}^{i, j}(m)+\sum_{\left\{j \mid(j, i) \in E_{s}\right\}} \sum_{\left\{n \mid G_{n} \in \Phi\right\}} \sum_{m=1}^{L_{n}} r_{n}^{j, i}(m)\right)
\end{gathered}
$$

Moreover, the algorithm makes an auxiliary off-peak substrate topology $G_{s}^{T}$. At the first, $G_{s}^{T}$ is the same as the substrate network topology. 


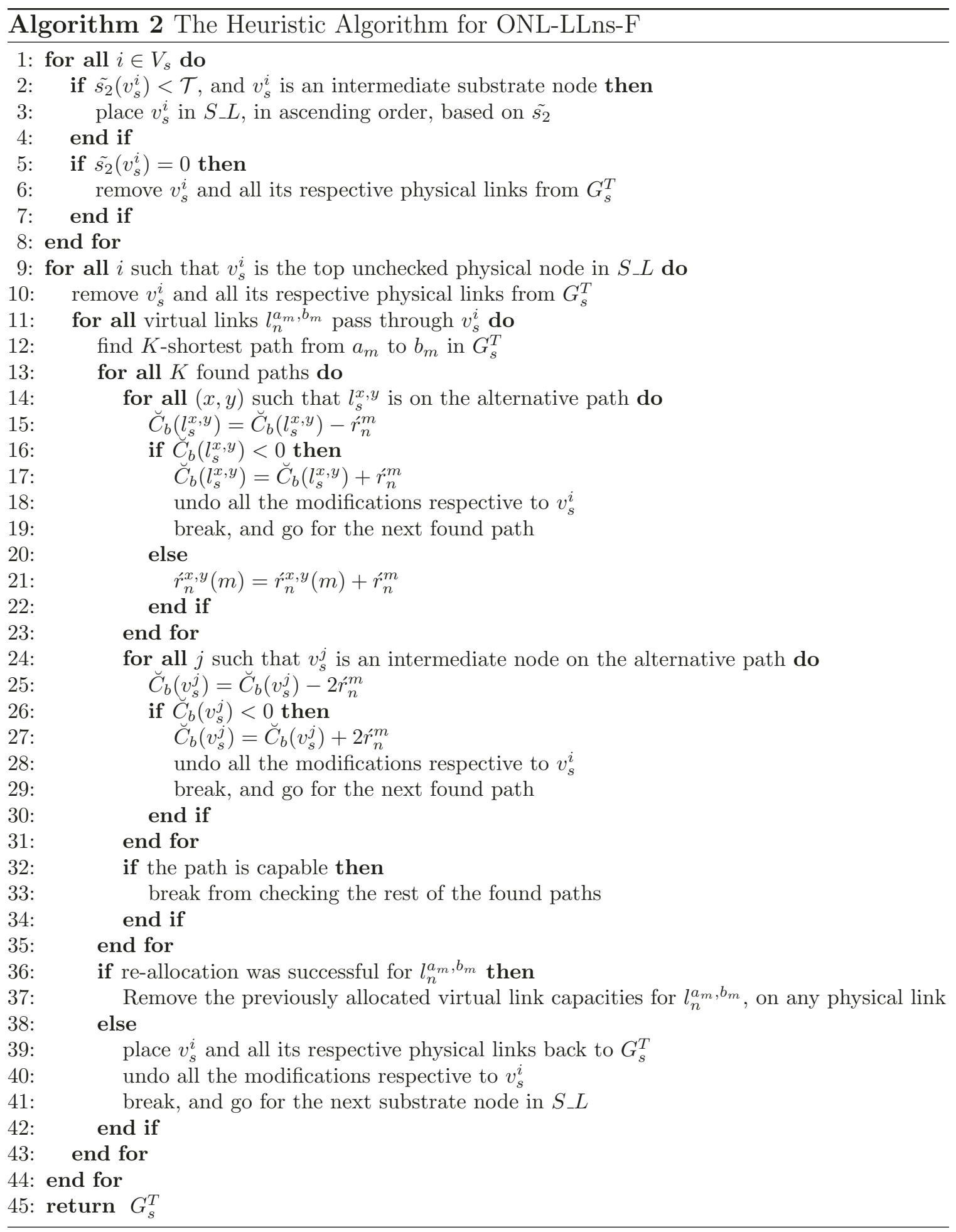

By knowing the calculated metrics, the algorithm sorts all the intermediate substrate nodes with the stress rate of less than $\mathcal{T}$. Since we do not reconfigure the 
mapping of virtual nodes in ONL-LLns-F, the heuristic sets only capable intermediate nodes into the sleep mode. In this regard, because the substrate nodes with the larger number of involved VNs are more essential for connectivity and bandwidth demands, the algorithm starts setting intermediate physical nodes into the sleep mode from the node that has the smaller number of involved VNs, and lower off-peak utilization. This happens when the algorithm sorts the intermediate physical nodes with $\tilde{s_{2}}\left(v_{s}^{i}\right)<\mathcal{T}$, in ascending order based on $\tilde{s_{2}}$. The list is represented by $S_{-} L$. Note that the $\tilde{s_{2}}\left(v_{s}^{i}\right)$ threshold amount is adjustable. We study the impact of different values for $\tilde{s_{2}}$ threshold on the heuristic's outcome in Section 4.5

The next step checks the possibility of removing the intermediate physical nodes and their respective physical links. This step also ensures the rearranged network accommodates the customer's off-peak traffic demands. In this regard, the algorithm removes the top unchecked physical node $v_{s}^{i}$ in $S_{-} L$, and its respective physical links, from $G_{s}^{T}$. Nevertheless, there must be a single alternative path for every virtual link $l_{n}^{a_{m}, b_{m}}$ that was passing through the removed substrate node $v_{s}^{i}$. The algorithm finds $K$ loopless shortest paths from the source node of the virtual link to its sink node in $G_{s}^{T}$. Our preferred routing algorithm to find $K$ loopless shortest paths is Yen's algorithm [91], while the cost of every physical link is assumed to be 1 . Note that the value of $K$ is adjustable, and the effect of different values of $K$ on the heuristic's result is discussed in Section 4.5. The alternative path must support the off-peak traffic demand $r_{n}^{m}$ of the respective virtual link $l_{n}^{a_{m}, b_{m}}$. So, a capable alternative path needs to satisfy some constraints.

First, the unused off-peak bandwidth capacity $\breve{C}_{b}\left(l_{s}^{x, y}\right)$ of every physical link on the alternative path must be equal or greater than the off-peak traffic demand $r_{n}^{m}$ of the virtual link. Second, the unused off-peak bandwidth capacity $\breve{C}_{b}\left(v_{s}^{j}\right)$ of every physical node on the alternative path also must support the off-peak traffic demand $r_{n}^{m}$ of the virtual link. If the found path satisfies these constraints, then the algorithm updates $\breve{C}_{b}\left(l_{s}^{x, y}\right)$ and $\breve{C}_{b}\left(v_{s}^{j}\right)$ of all the physical links and nodes on the path, respectively, and stops checking the remaining of the shortest paths. Besides, it removes the previously allocated traffic capacities for the virtual link. However, if one or multiple physical nodes or links on the found path do not support the demanded traffic, then the heuristic checks the next shortest path. In the case there is no capable alternative path, the algorithm puts the physical node and its respective physical links back to $G_{s}^{T}$, discards the modifications, and checks the removal possibility of the next 
substrate node in $S_{-} L$.

After that all of the physical nodes in $S_{-} L$ are checked, $G_{s}^{T}$ is returned as the energy-efficient off-peak substrate topology.

As mentioned earlier, the defined BILP for off-peak node and link energy optimization by local link reconfiguration is $\mathcal{N} \mathcal{P}$-hard. Therefore, it is not scalable in the case of large network sizes, due to the long execution time. Nevertheless, it is expected the suggested heuristic for the same problem is considerably simpler and faster than the formulated BILP. The largest loop that starts in line 9 and ends in line 44 determines the complexity of the proposed heuristic. In this regard, the main loop that starts in line 9 is run for each physical node, so its complexity is $O\left(\left|V_{s}\right|\right)$. The first sub-loop that starts in line 11 is run for every virtual link belonging to all of the VNs, which passes through the physical node. Therefore, its complexity is $O\left(|\Phi|\left|E_{v}^{m}\right|\right)$, where $E_{v}^{m}$ is the set of edges of the involved virtual network with the largest number of virtual links. The heuristic calls Yen's algorithm in line 12. The complexity of Yen's algorithm is $O\left(K\left|V_{s}\right|\left(\left|E_{s}\right|+\left|V_{s}\right| \log \left|V_{s}\right|\right)\right.$. The second sub-loop that starts in line 13 is run for $K$ found shortest paths, and consequently its complexity is $O(K)$. The third subloop starting at line 14 checks the capability of every physical link on the found path. So, its complexity is $O\left(\left|E_{s}\right|\right)$. The other sub-loop that runs beside the previous one, checks the eligibility of physical nodes on the found path, and therefore its complexity is $O\left(\left|V_{s}\right|\right)$. The heuristic might need to check all the virtual links' capacities in every physical link, in the worst-case scenario, in order to discard the capacity and traffic modifications for each rerouted virtual link. So, the complexity of the undoing function in lines 18, 28 and 40 is $O\left(\left|E_{s}\right||\Phi|\left|E_{v}^{m}\right|\right)$. Hence, the complexity of the proposed heuristic is $O\left(K\left|V_{s}\right||\Phi|\left|E_{v}^{m}\right|\left(\left|V_{s}\right|^{2} \log \left|V_{s}\right|+\left|E_{s}\right|^{2}|\Phi|\left|E_{v}^{m}\right|+\left|V_{s}\right|\left|E_{s}\right||\Phi|\left|E_{v}^{m}\right|\right)\right)$. Consequently, the proposed heuristic algorithm is considerably simpler than the BILP, and it could be solved in a polynomial time.

\subsection{Evaluation}

The proposed energy-saving solutions are supposed to reduce the total node and link energy consumption in a VNE during the off-peak time. They need to guarantee the full connectivity, the off-peak bandwidth requirements, and also the off-peak processing demands. Several random VNE setups have been assessed to study the impact of different parameters on the energy-saving capability of the discussed solutions. 
Recently, Waxman algorithm [84] is widely used by the researchers to generate random topologies for VNEs [17,69,85-87]. Therefore, in this chapter, substrate and virtual networks' topologies are generated by Waxman algorithm. Waxman generates random network topologies based on two parameters, $\tilde{\lambda}$, and $\tilde{\mu}$. As $\tilde{\lambda}$ grows the probability of having an edge between any pair of nodes in the topology is increased. As $\tilde{\mu}$ grows there is a larger ratio of long edges to short edges. In this chapter, we choose the Waxman parameters, for both substrate and virtual networks' topologies, as $\tilde{\lambda}=0.5$ and $\tilde{\mu}=0.5$, in the area size of $100 \times 100$. After creating random substrate and virtual networks' topologies, a substrate links' bandwidth capacity and a virtual link' peak demand are generated randomly with the uniform distribution. The bandwidth capacity of each physical link is a random amount between 100Mbps and 200Mbps, but each virtual link's bandwidth demand is generated randomly between 50Mbps and 100Mbps. Therefore, according to [56], the base and the maximum physical link power consumption for any physical link is $0.9 \mathrm{Watt}$, and 1.00Watt, respectively. The bandwidth capacity of every physical node is a constant amount of 1 Gbps. Besides, the processing capacity of physical and virtual nodes are generated randomly with the uniform distribution. The processing capacity of each substrate node is a random amount between $500 \mathrm{Mhz}$ and $800 \mathrm{Mhz}$, but every virtual link's processing demand is generated randomly between $400 \mathrm{Mhz}$ and $500 \mathrm{Mhz}$. The randomly generated substrate networks are symmetric, so if there is a physical link from node $i$ to node $j$ with a specific amount of bandwidth capacity, then there is also a physical link from node $j$ to node $i$ with the same amount of bandwidth capacity. In the next step, each created virtual node is mapped onto a substrate node belonging to the set of substrate node candidates that have enough processing capacity for that virtual node, with the uniform distribution. Afterwards, every generated virtual link's peak bandwidth demand is allocated in a substrate path through a state-of-the-art algorithm that does not concern with energy efficiency.

As it is discussed, the formulated ILPs are $\mathcal{N} \mathcal{P}$-hard, so they are not scalable for large network sizes. Therefore, we assess the capability of the defined ILPs on small random simulation setups, similar to the other related works in $[17,56,69,70]$. The scale of a small random simulation setup is comparable to GEANT network that is a real universal network. GÉANT has 22 nodes and 36 bidirectional links. The ILPs are solved by CVX package [92] that uses MOSEK solver [88]. Nonetheless, the theoretical complexity analysis reveals the proposed heuristic algorithm is considerably simpler, 
and therefore it is scalable for large network sizes. Hence, the performance of the suggested heuristic is examined on medium random simulation setups.

Every small random simulation setup contains 10 randomly generated VNEs. Each VNE in a small random simulation setup has at least 2 random virtual networks that are allocated in a single random substrate network, while the substrate network has 15 nodes and each virtual network has 5 nodes. The average number of physical links in the small random simulation setups is 55. Furthermore, every medium random simulation setup includes 10 randomly generated VNEs. All the VNEs in a medium random simulation setup have at least 2 random virtual networks that are mapped onto a single random substrate network, while the substrate network has 50 physical nodes and each virtual network has 20 virtual nodes. The average number of physical links in the medium random simulation setups is 570 . Note that the average results including confidence intervals with the confidence level of $90 \%$ are calculated for each setup. A confidence interval is calculated by Equation 3.22.

\subsubsection{ILPs}

First, we study the power saving capability of the formulated MILPs for ONL-LNLs-F and ONL-LNLs-SP, on a small random simulation setup. We measured a network's total node and link power consumption for different off-peak ratios, before and after applying the defined solutions. An off-peak ratio is the fraction of a network's off-peak traffic rate by its peak traffic rate. In the case the off-peak ratio is $x$, the off-peak demands of every virtual network is $x$ times its peak demands. The measurement results for the off-peak ratio range of 0.1 to 0.9, are shown in Figure 4.1. Note that we measure power consumption instead of energy consumption, because the amount of energy consumed depends on the total time we measured, and so it is not a good metric to compare.

The results reveal the formulated MILPs for ONL-LNLs-F and ONL-LNLs-SP reduce the total node and link power consumption of the simulated virtualized net-

works, noticeably. Besides increasing the off-peak traffic ratio increases the amount of traffic that needs to be re-allocated, so the programs are more limited in terms of finding alternative paths for each removed virtual link. In result, the number of physical nodes and links in the sleep mode is decreasing by increasing the off-peak traffic demand, and therefore, as Figure 4.1 confirms, the programs could save lower amounts of power. Note that the total power consumption in the case of Semi-Proportional 
power model is changing by varying the off-peak ratio, even before applying any energy-saving solution. However, because a larger portion of a node or link's power consumption in Semi-Proportional power model, is for keeping the device active $\left(\tilde{p}^{b}\right)$, the variations in the total power consumption due to the traffic fluctuations are small and could not be observed in the scale of Figure 4.1.

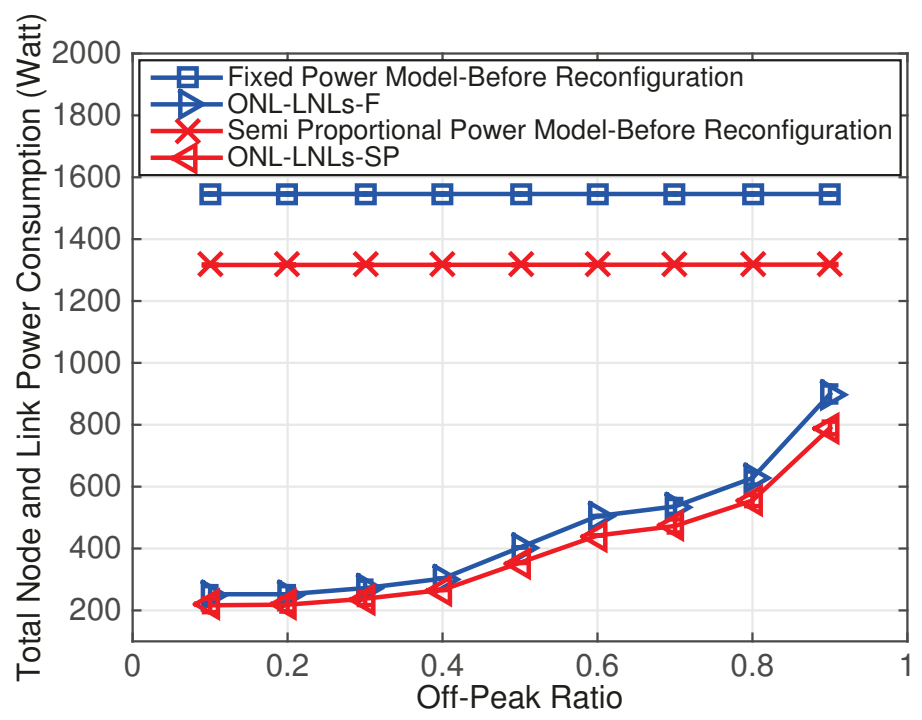

Figure 4.1: The total node and link power consumption based on the off-peak ratio by the MILPs for ONL-LNLs-F and ONL-LNLs-SP

According to the defined power models in Section 4.2, physical nodes consume more energy than physical links. Thus, it is expected a larger portion of energy we could save in a VNE is achieved by reducing the energy consumption of substrate nodes rather than substrate links. In this regard, we calculated the total amount of power saved in the network by the formulated MILP for ONL-LNLs-F. Besides, we also calculated the total saved power in physical nodes, and total saved power in physical links by the same MILP. Figure 4.2 shows the results for these measurements. Figure 4.2 confirms that a larger portion of total saved power is achieved by reducing the physical nodes' energy consumption rather than physical links. For instance, the formulated MILP for ONL-LNLs-F saved 1142.3Watt in total, when the off-peak traffic ratio is 0.5 . Nonetheless, 1100.3 Watt is saved by reducing the physical nodes' power consumption, but only 42.0 Watt is saved by decreasing the power consumption in physical links. 


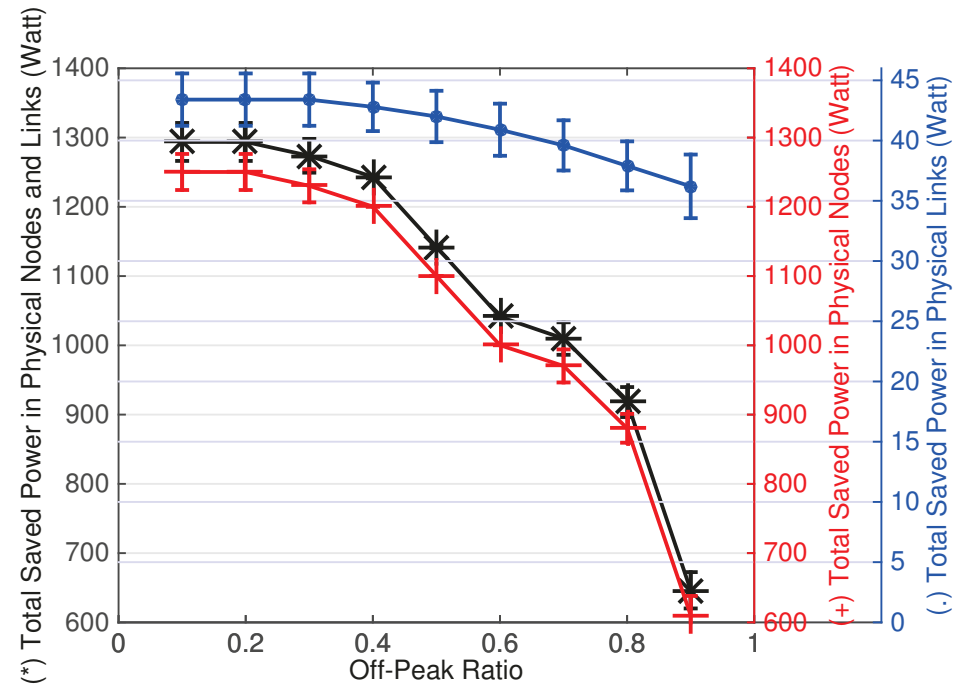

Figure 4.2: The total saved power in the network, the total saved power in physical nodes, and the total saved power in physical links based on the off-peak ratio by the MILP for ONL-LNLs-F

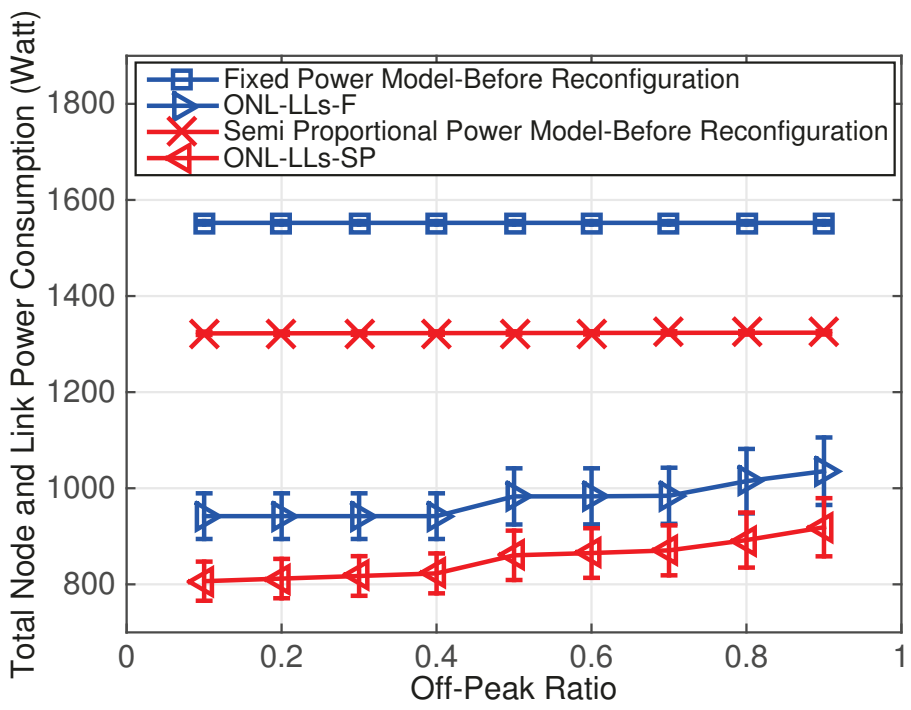

Figure 4.3: The total node and link power consumption based on the off-peak ratio by the MILPs for ONL-LLs-F and ONL-LLs-SP

Besides, Figure 4.3 and Figure 4.4 demonstrate that the network's total node and link power consumption before and after applying off-peak node and link energy optimization by local link reconfiguration programs, in the case of splittable traffic, and non-splittable traffic, respectively. These measurements are performed for different off-peak ratios, on a small random simulation setup. The programs decrease a VNE's 
node and link energy consumption by reconfiguring only some of the virtual links. The results prove that the formulated programs are able to save the power effectively, for either splittable traffic or non-splittable traffic, based on both power models.

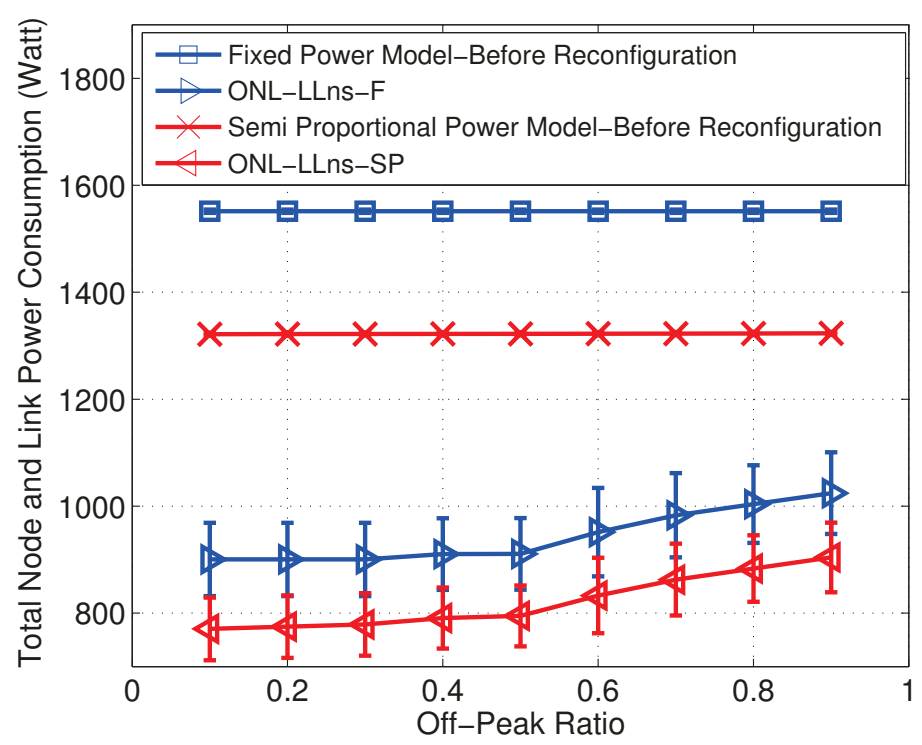

Figure 4.4: The total node and link power consumption based on the off-peak ratio by the BILPs for ONL-LLns-F and ONL-LLns-SP

The off-peak node and link energy optimization by local node and link reconfiguration modifies the mapping for some of the both virtual nodes and links. Nonetheless, the off-peak node and link energy optimization by local link reconfiguration adjusts the mapping for only some of the virtual links. Hence, it is expected the formulated programs for the first strategy save more energy than the defined programs for the latter method. In this regard, we tested the formulated MILPs for ONL-LNLs-F and ONL-LLs-F, on a small random simulation setup. Figure 4.5 illustrates that the percentage of power the formulated MILPs for ONL-LNLs-F and ONL-LLs-F could save on the range of off-peak ratio. The results confirm that the formulated MILP for ONL-LNLs-F could save larger amounts of power in comparison to the defined MILP for ONL-LLs-F. Although the local link reconfiguration strategy does not reconfigure virtual nodes and therefore it saves smaller amounts of power in comparison to the local node and link reconfiguration method, it causes fewer interruptions to the normal network operations. Note that, as Figure 4.5 reveals, the energy-saving ability of ONL-LNLs-F is more sensitive to the off-peak ratio fluctuations in comparison to the 
outcome of ONL-LLs-F. ONL-LNLs-F requires supporting both off-peak processing and traffic demands, but since ONL-LLs-F does not modify virtual nodes mapping, it only needs to handle the off-peak traffic demands. Therefore, the off-peak ratio variations have a greater impact on the energy-saving capability of ONL-LNLs-F rather than ONL-LLs-F.

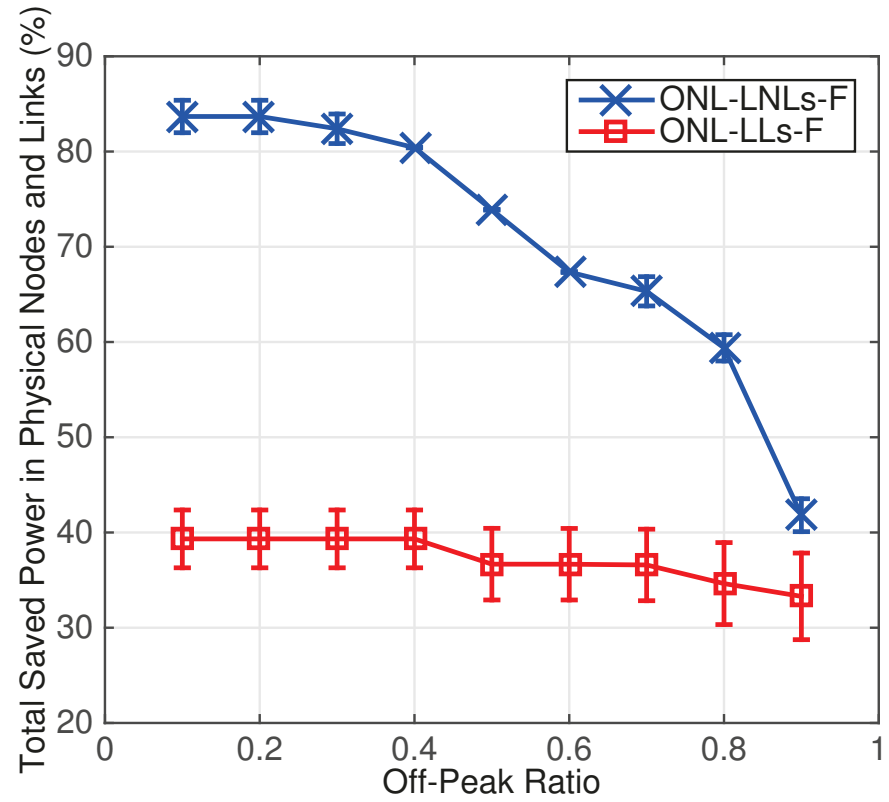

Figure 4.5: The total saved power in physical nodes and links based on the off-peak ratio by the MILPs for ONL-LNLs-F and ONL-LLs-F

It is expected the programs save larger amounts of power when traffic is splittable, because they are more flexible in terms of finding alternative paths. We checked this issue on the formulated ILPs for off-peak node and link energy optimization by local link reconfiguration. Figure 4.6 shows the percentage of power the formulated ILPs for ONL-LLs-F and ONL-LLns-F could save in the defined range of off-peak ratio, on a small random simulation setup. The results prove that the programs could save more power when traffic is splittable in comparison to when traffic is non-splittable. Note that ONL-LLs-F saves more power than ONL-LLns-F in the high off-peak ratios. This is because, when off-peak ratio is low, it is easier for the programs to find the alternative paths, and therefore ONL-LLs-F and ONL-LLns-F have almost the same outcome. However, when off-peak ratio is high, it is more probable for the programs to find the replaced paths by splitting high traffic loads into smaller loads. 


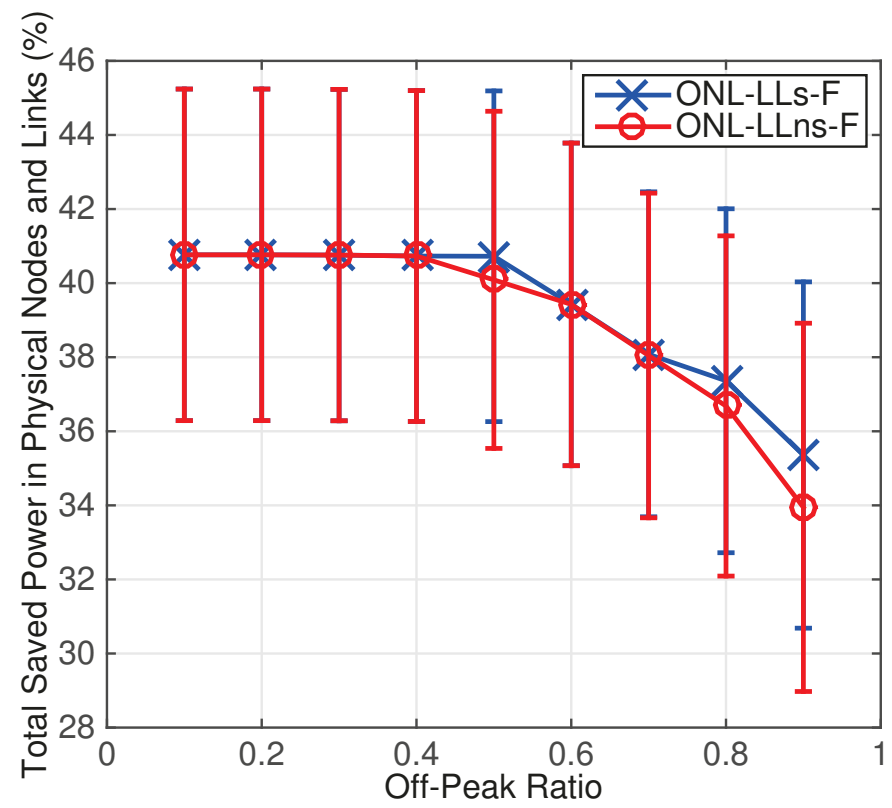

Figure 4.6: The total saved power in physical nodes and links based on the off-peak ratio by the ILPs for ONL-LLs-F and ONL-LLns-F

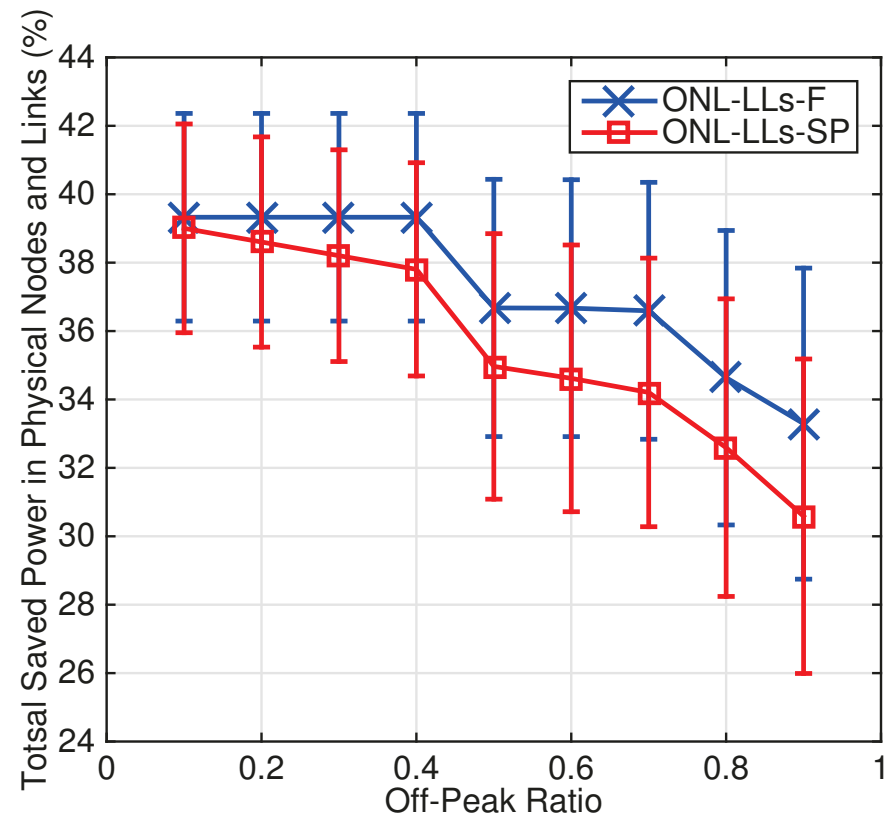

Figure 4.7: The total saved power in physical nodes and links based on the off-peak ratio by the MILPs for ONL-LLs-F and ONL-LLs-SP

Moreover, it is expected to save higher levels of power when Fixed power model is 
applied, in comparison to when Semi-Proportional power model is employed, mainly due to two reasons. First, a physical node or link, that is not fully utilized, consumes a smaller amount of power when it is designed based on Semi-Proportional power model, in comparison to when it is designed based on Fixed power model. Therefore, by setting the same physical nodes and links into the sleep mode, the total amount of saved power is smaller in the case of Semi-Proportional power model. Second, if the energy-saving program sets a physical node into the sleep mode, then it re-allocates its virtual nodes to other substrate nodes, and re-maps its respective virtual links to alternative paths. In the case of Semi-Proportional power model, the re-allocated virtual node and the rerouted traffic increase the power consumption over the alternative physical nodes and links. However, in the case of Fixed power model, since the traffic load does not affect a node or link's power consumption, the reconfiguration does not increase the power consumption over the alternative physical nodes and links. We tested the formulated MILPs for ONL-LLs-F and ONL-LLs-SP on a small random simulation setup in order to verify this expectation. The results in Figure 4.7 reveals the percentage of saved power by MILPs for ONL-LLs-F and ONL-LLs-SP. Figure 4.7 confirms that the MILP for ONL-LLs-F could save larger amounts of power than the MILP for ONL-LLs-SP.

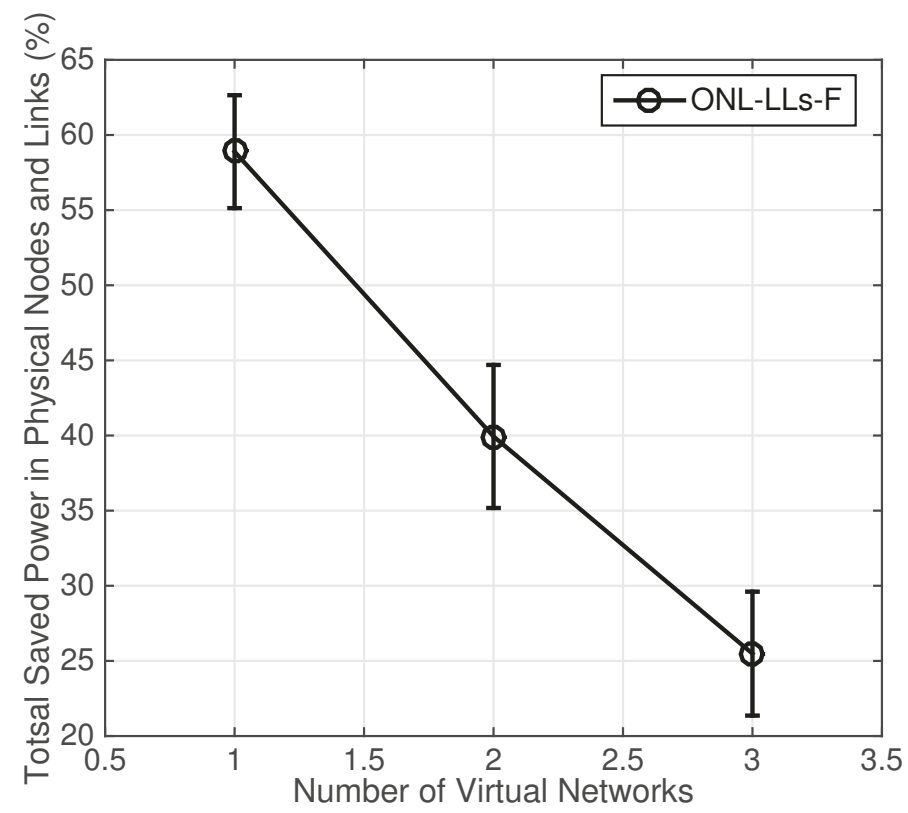

Figure 4.8: The total saved power in physical nodes and links based on the number of involved virtual networks by the MILP for ONL-LLs-F 
Furthermore, it is necessary to analyze the impact of different numbers of allocated virtual networks over a substrate network, on the energy-saving ability of the proposed solutions. In this regard, we measured the percentage of power the formulated MILP for ONL-LLs-F could save on a small random simulation setup, when 1, 2 or 3 randomly generated virtual networks are allocated in the substrate network. The off-peak ratio is assumed to be 0.5. The results are presented in Figure 4.8. Figure 4.8 reveals that increasing the number of allocated virtual networks in the substrate network, decreases the total amount of power the program could save. Allocating a new virtual network in a substrate network imposes several constraints to the programs, as new virtual nodes and links are mapped onto the physical nodes and links. For instance, if a program wants to set a physical node into the sleep mode, then it needs to re-allocate all the virtual nodes mapped onto the physical node, as well as every virtual link passing the physical node. By allocating a new virtual network, the program requires to find alternative physical nodes and paths for the newly mapped virtual nodes and links. Therefore, the program is more limited, and its energy-saving capability is degraded.

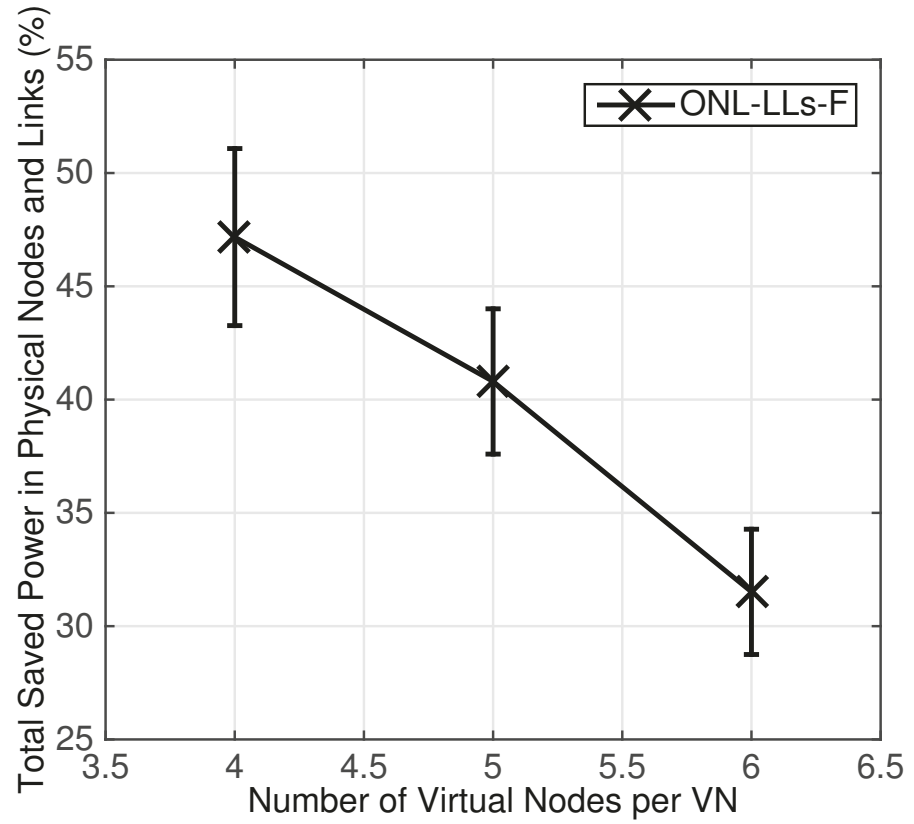

Figure 4.9: The total saved power in physical nodes and links based on the number of virtual nodes per VN by the MILP for ONL-LLs-F

It is also important to study the effect of different numbers of virtual nodes per 
virtual network, on the energy-saving ability of the advised solutions. The number of virtual nodes per VN expresses the size of virtual networks. Accordingly, we probed the percentage of power saved by the formulated MILP for ONL-LLs-F on a small random simulation setup, while the number of virtual nodes per VN varies between 4 and 6 . The off-peak ratio is assumed to be 0.5 . The results are displayed in Figure 4.9. Increasing the number of virtual nodes per $\mathrm{VN}$, raises the number of allocated virtual nodes in the physical nodes. Besides, adding virtual nodes in a virtual network increases the number of virtual links in that virtual network. Consequently, incrementing the number of virtual nodes per $\mathrm{VN}$ imposes several new constraints to the proposed energy-saving solutions, and makes them more limited in terms of setting physical nodes and links into the sleep mode. Hence, as it is confirmed in Figure 4.9 for ONL-LLs-F, the program save smaller amounts of power when the number of virtual nodes per $\mathrm{VN}$ is increased.

\subsubsection{The Heuristic}

On the other hand, it is essential to study the effectiveness of the proposed heuristic algorithm for ONL-LLns-F. In this regard, first, we tested the heuristic algorithm on a medium random simulation setup, and measured the network's total node and link power consumption before and after applying the heuristic algorithm, on the defined range of off-peak ratio.

As discussed in Section 4.4, $K$ affects the heuristic's outcome. $K$ is the number of shortest paths the algorithm examines in order to find a single capable alternative path for each re-mapped virtual link. The heuristic is simulated when $K$ is $1,2,3,4$ or 5. The results are presented in Figure 4.10. The results confirm that the proposed heuristic algorithm is able to reduce a VNE's total node and link power consumption, effectively. Besides, Figure 4.10 reveals that increasing the off-peak ratio decreases the amount of power the heuristic could save. This is because increasing the off-peak ratio raises the traffic load algorithm needs to re-allocate, and therefore the heuristic is more limited in terms of finding alternative paths.

In addition, Figure 4.10 verifies increasing $K$ raises the amount of power the heuristic could save. If the heuristic needs to re-map a virtual link, then it finds $K$ shortest paths from the source node to the sink node of the virtual link in order to provide a single qualified alternative path. However, the alternative path requires satisfying some capacity constraints. In this regard, the shortest path $(K=1)$ might 
not persuade the capacity constraints, but the second $(K=2)$ or third $(K=3)$ shortest path may do. Consequently, the heuristic might save more power when $K$ is increased. Nevertheless, incrementing $K$ increases the heuristic's run time, because it needs to find larger number of shortest paths. This is discussed theoretically in Section 4.4. Besides, considering a specific off-peak ratio in Figure 4.10, the variations in the saved amount of power by heuristic is decreasing when $K$ is increasing. This is because the number of available paths between two nodes in a substrate network is limited, and by incrementing $K$ the majority of possible paths are considered by the heuristic, so it could not find new replaced paths. In consequence, a large enough value of $K$, based on the substrate network size, makes it probable for the heuristic to save the maximum amount of power in a VNE.

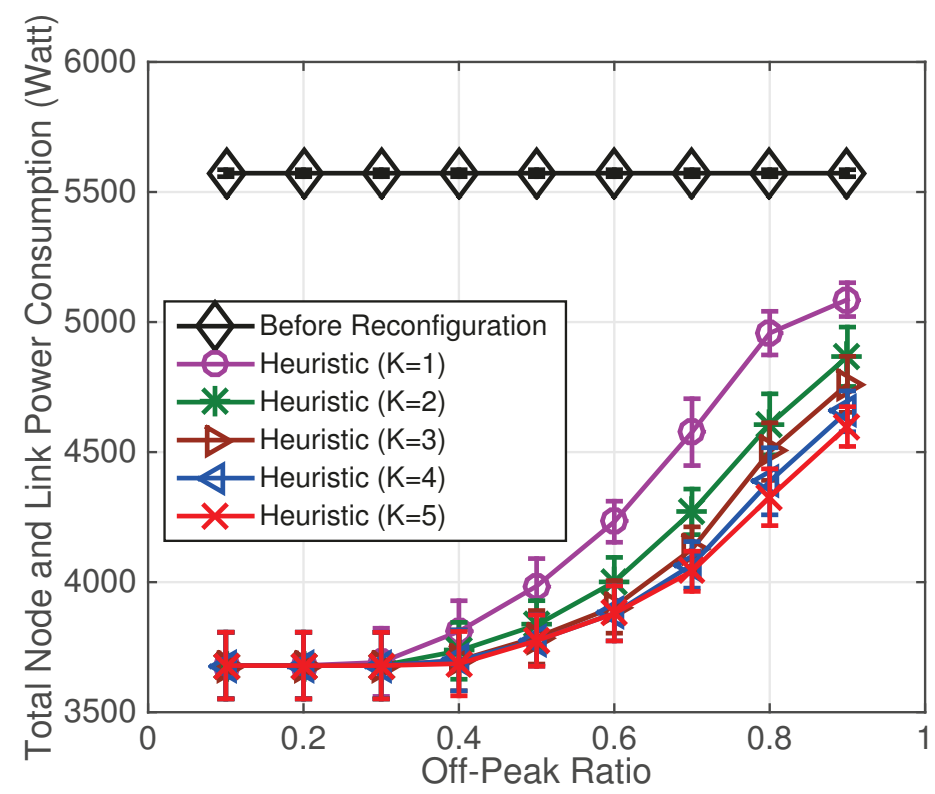

Figure 4.10: The total node and link power consumption based on the off-peak ratio before and after applying the heuristic with different values of $K$, for ONL-LLns-F

It is vital to check the difference between the outcome of the formulated BILP for ONL-LLns-F as the optimum result, and the proposed heuristic for the same problem. This is tested on a small random simulation setup, and results are displayed in Figure 4.11. Figure 4.11 shows the percentage of power the BILP for ONL-LLns-F saves, as well as the percentage of power the proposed heuristic for the same problem with different values of $K$ could save, on the defined range of off-peak ratio. Similar to 
the results of previous simulation setup, increasing the off-peak ratio degrades the ability of the BILP and the heuristic in terms of saving the power. Moreover, the heuristic works closely to the optimum points set by the BILP, while the heuristic is significantly faster than the BILP.

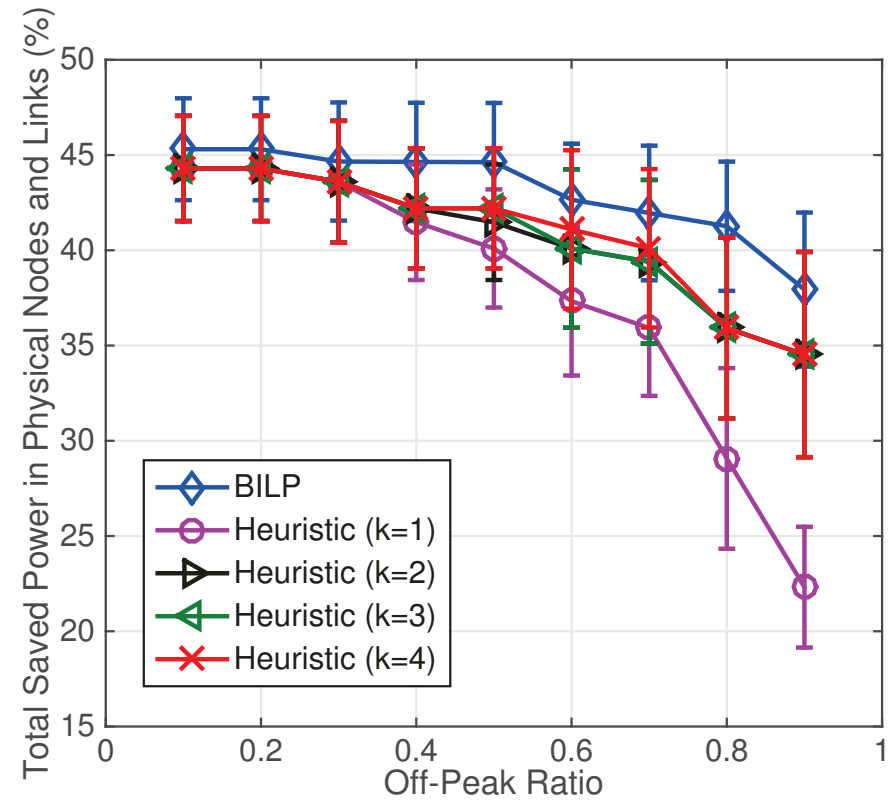

Figure 4.11: The total saved power in physical nodes and links based on the off-peak ratio by the BILP and the heuristic with different values of $K$, for ONL-LLns-F

Additionally, we evaluated the impact of different numbers of mapped virtual networks on a substrate network, on the heuristic's outcome when $K$ is 5 and the offpeak ratio is 0.5 . The number of allocated VNs is ranged from 1 to 3. The outcome is presented for a medium random simulation setup in Figure 4.12. Similar to the results shown in Figure 4.8, the results in Figure 4.12 confirms that increasing the number of allocated virtual networks in the substrate network, limits the heuristic to find replaced paths, and in consequence decreases the amount of power it could save.

The influence of different numbers of virtual node per virtual network, on the heuristic's outcome is also tested over a medium random simulation setup, and the results are displayed in Figure 4.13. The off-peak ratio and $K$ are assumed to be 0.5, and 5, respectively. Similar to the results displayed in Figure 4.9, the results in Figure 4.13 verifies that incrementing the number of virtual nodes per mapped VN, degrades the ability of the heuristic in terms finding alternative paths, and therefore decreases the amount of power the heuristic could save. 


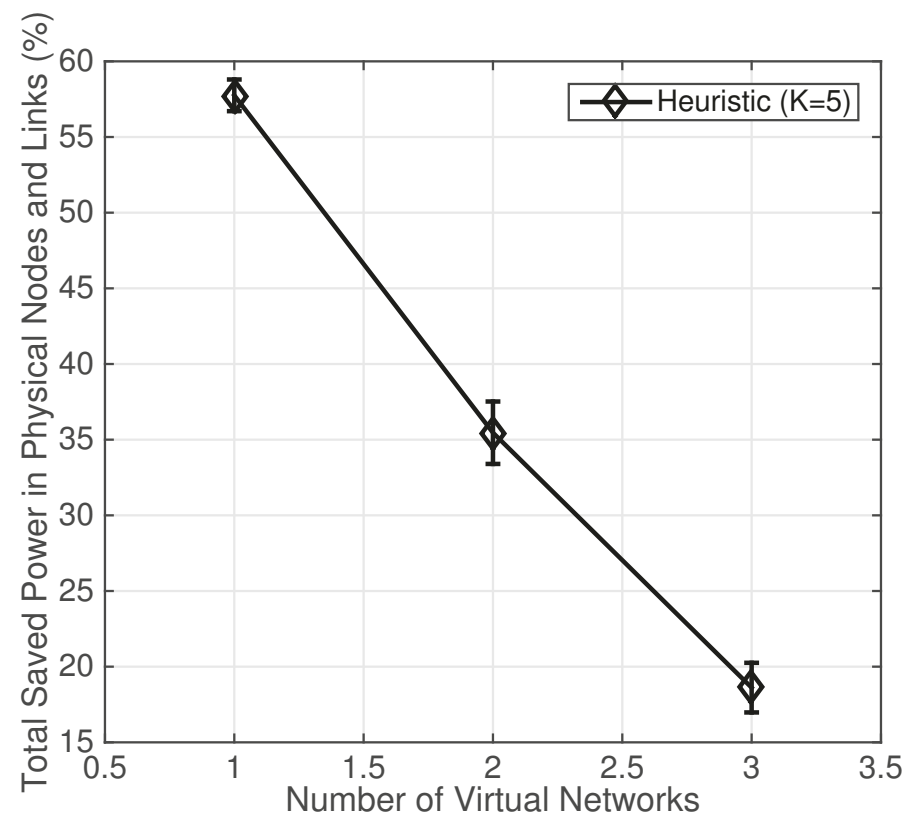

Figure 4.12: The total saved power in physical nodes and links based on the number of involved virtual networks by the heuristic with $K=5$, for ONL-LLns-F

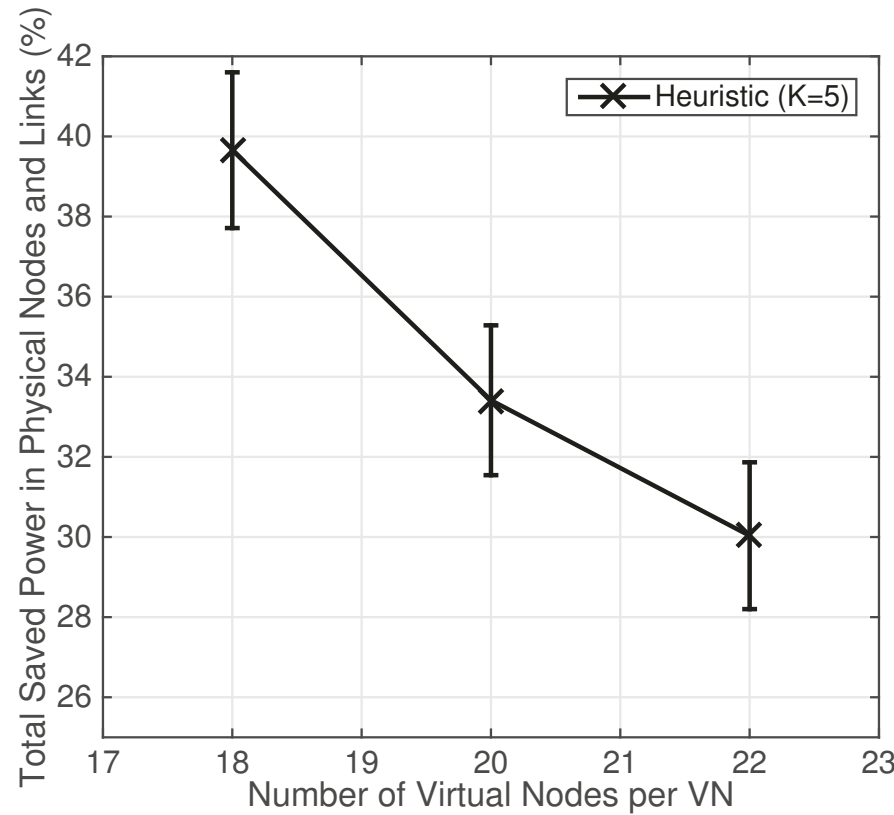

Figure 4.13: The total saved power in physical nodes and links based on the number of virtual nodes per VN by the heuristic with $K=5$ for ONL-LLns-F

Moreover, it is explained in Section 4.4 that the proposed heuristic, similar to the 
formulated BILP for ONL-LLns-F, does not sleep the physical nodes with $\tilde{s_{2}}\left(v_{s}^{i}\right) \geq$ $\mathcal{T}$, in order to decrease the traffic disruptions due to reconfiguration. Figure 4.14 studies the effect of changing $\tilde{s_{2}}$ threshold on the capability of the heuristic when $K=5$, over a medium random simulation setup. The off-peak ratio is assumed as 0.5. Figure 4.14 shows that decreasing $\tilde{s_{2}}$ threshold amount, decreases the amount of power heuristic saves, because a smaller number of substrate nodes and links are considered for power saving. Although decreasing $\tilde{s_{2}}$ threshold decreases the amount of power formulated solutions could save, it reduces the traffic interruptions to the normal network operations, due to the reconfiguration.

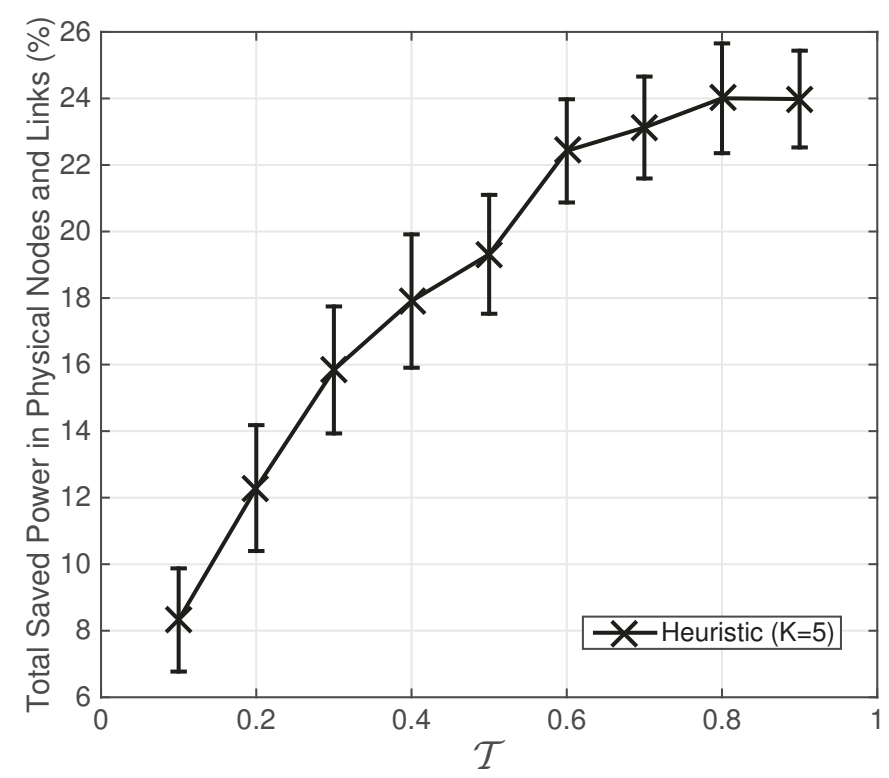

Figure 4.14: The impact of different $\tilde{s_{2}}$ thresholds on the total saved power in physical nodes and links by the heuristic when $K=5$ for ONL-LLns-F

It is expected this problem saves a larger amount of power in comparison to the previously proposed solutions in Chapter 3. This is because the suggested methods in Chapter 3 only concerns about a VNE's link energy consumption, while physical nodes consume more energy. We tested the proposed heuristic in this chapter and the suggested heuristic in Chapter 3, on a medium simulation setup. The results in Figure 4.15 illustrate that the suggested heuristic in this chapter could save significantly higher rates of the total power than the previously proposed heuristic. 


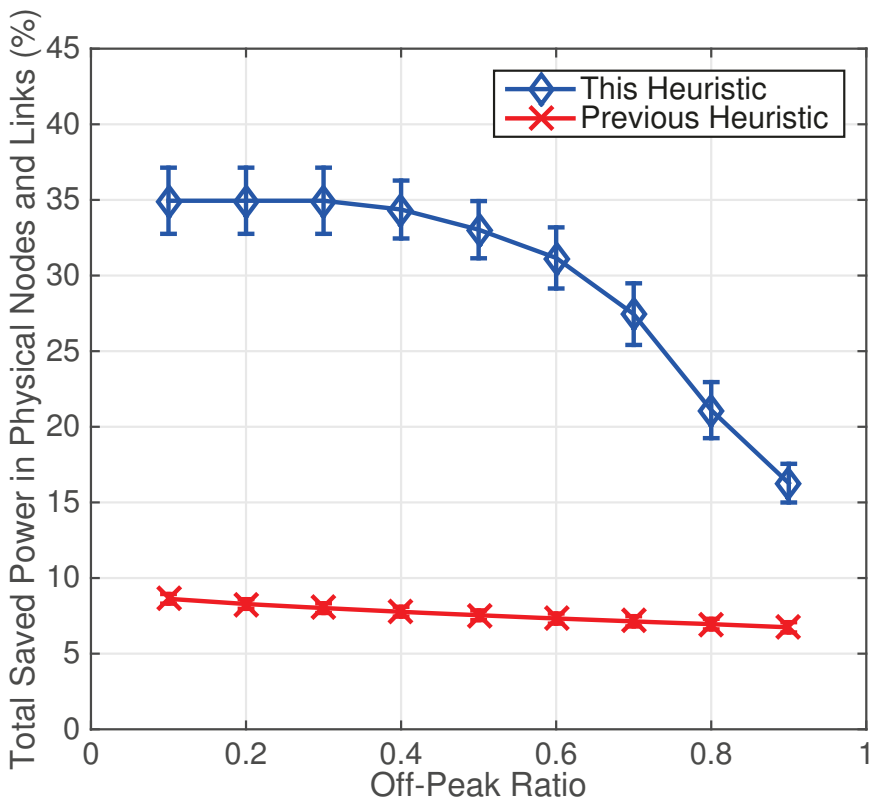

Figure 4.15: Comparison between the suggested heuristic in this chapter and previously proposed heuristic in Chapter 3

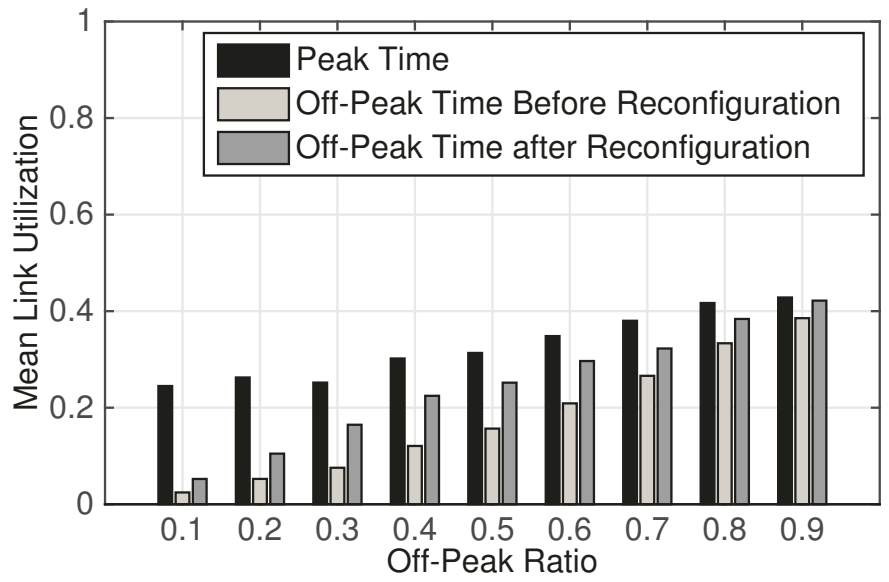

Figure 4.16: The mean link utilization based on the off-peak ratio over different configurations

Besides, rerouting the traffic to the other substrate paths causes changes to the links' utilization. The heuristic algorithm re-maps the off-peak traffic onto the other paths in order to save energy. Accordingly, it is needed to make sure the increased utilization is controlled and does not cause congestion. The link utilization for three different configurations is measured on a medium random simulation setup, and the 
average results are shown in Figure 4.16. The first configuration is for the peak time when the allocated bandwidth is able to handle "Worst-Case" scenarios. The second configuration is for the off-peak period while no energy-saving algorithm is implemented. Over this period the links are less utilized while the same bandwidth capacity is allocated and consume the same power as the peak time. After applying our suggested heuristic when $K=5$ and the off-peak ratio is 0.5 , the average link utilization is increased, but it is still less than the maximum utilization. Note that the link utilization shown in Figure 4.16 is the mean of the maximum points of all the test scenarios.

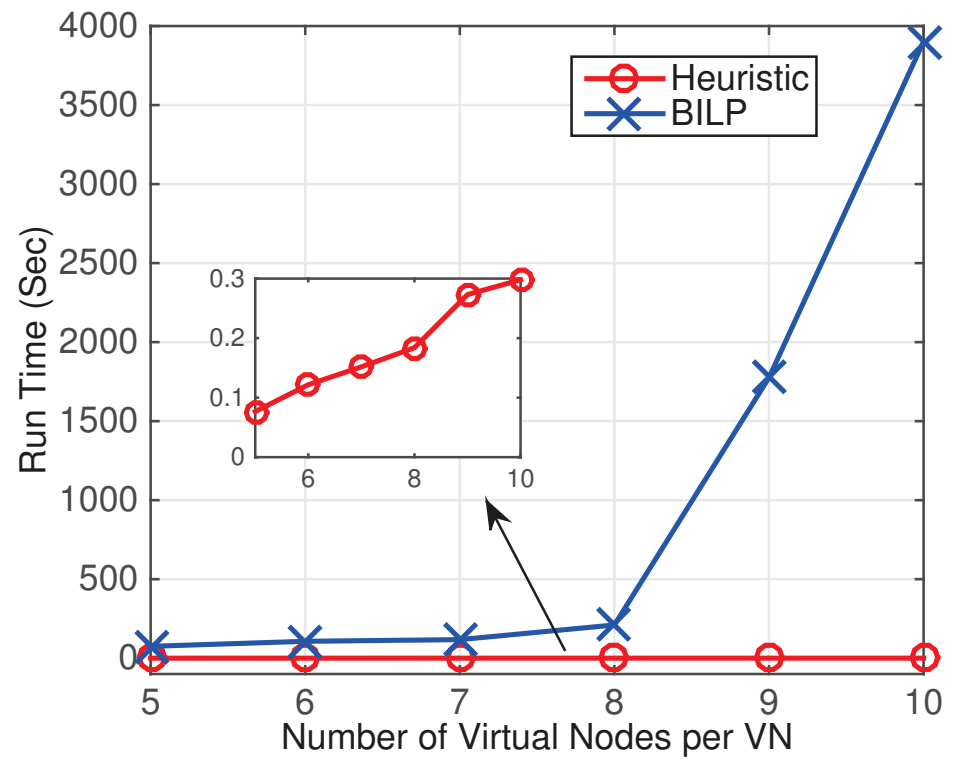

Figure 4.17: Required run time based on the different number of virtual nodes per $\mathrm{VN}$, for the BILP and the heuristic with $K=5$, for ONL-LLns-F

The formulated BILP for ONL-LLns-F is $\mathcal{N} \mathcal{P}$-hard, while the complexity of the proposed heuristic algorithm for the same problem is $O\left(K\left|V_{s}\right||\Phi|\left|E_{v}^{m}\right|\left(\left|V_{s}\right|^{2} \log \left|V_{s}\right|+\right.\right.$ $\left.\left.\left|E_{s}\right|^{2}|\Phi|\left|E_{v}^{m}\right|+\left|V_{s}\right|\left|E_{s}\right||\Phi|\left|E_{v}^{m}\right|\right)\right)$. So, it is expected the proposed heuristic needs less run time in comparison to the formulated binary integer linear program for the same problem. We verified run time for the BILP and the heuristic on a single random scenario. This scenario includes a random substrate topology with 15 physical nodes, while 2 random virtual networks are mapped onto the substrate network, and the off-peak ratio is assumed to be 0.5. We probed the required run time for the BILP and the heuristic with $K=5$, while the number of virtual nodes per virtual network 
is changing from 5 to 10 . The number of virtual nodes per VN expresses the size of virtual networks. As the results in Figure 4.17 show, the heuristic needs considerably lower run time in comparison to the BILP. Besides, Figure 4.17 confirms that enlarging the size of mapped virtual networks, increases the required run time for both the heuristic and the BILP. Note that the number of nodes per virtual network affects the number of virtual links per virtual network, and this impacts the time complexity of the heuristic. However, since the BILP is $\mathcal{N} \mathcal{P}$-hard, its required run time changes almost exponentially by changing the number of virtual nodes per VN.

The simulation results prove the suggested energy-saving solutions are able to reduce a VNE's node and link energy consumption, during the off-peak period, effectively. Besides, the proposed heuristic is a simple and fast algorithm that works closely to the optimum points.

Note that every simulation setup is quite large to cover a substantial number of random topologies in order to verify the effectiveness of the proposed solutions. Besides, the calculated confidence intervals confirm that the results are precise enough to reveal the significances of the different energy-saving methods.

\subsection{Summary}

In this chapter, we have formulated multiple novel ILPs that optimize the node and link energy consumption in a VNE during the off-peak period, by reconfiguring the mapping for some of the mapped virtual nodes and links. They are developed according to two power models, and considering the impact of traffic splittability. Because the formulated ILPs are $\mathcal{N} \mathcal{P}$-hard, we also suggested a novel energy-saving heuristic algorithm. The defined ILPs and the heuristic are tested over randomly generated VNEs. Simulation results show that the proposed solutions are noticeably effective and the heuristic works closely to the optimum points. 


\section{Chapter 5}

\section{GreenMap: Green Mapping of Heterogeneous MapReduce-based Virtual Networks onto a Data Center Network and Controlling Incast Queueing Delay}

\section{$5.1 \quad$ Introduction}

The cloud computing is becoming widespread, and the energy consumption of the physical infrastructure that provides resources for a cloud is growing [93]. Accordingly, the energy management is a key challenge for data centers to reduce all their energyrelated costs [94].

MapReduce [95] is a cloud computing approach that parallelizes a computation across a large-scale cluster of servers. We target MapReduce, because it is widely deployed in many data centers like Google [96], Yahoo!, Amazon, and Facebook [97]. More than ten thousands distinct MapReduce programs, with the average of one hundred thousand MapReuce jobs per day, have been executed at Google [96]. In this framework, users specify the computation tasks by generating map and reduce functions. Nodes that perform a mapping job are called mappers. Mappers process input data and generate intermediate key and value pairs. The generated key and value pairs are shuffled through shuffler nodes to the other nodes that perform a reducing job, called reducers. Reducers aggregate the received intermediate key and value pairs from different mappers and compute the computational results for an application.

VNs together with VMs underpin Virtualized Data Centers (VDCs). A VDC 
in the virtual layer consists of virtual networks in which VMs are connected with virtual links by virtual switches/routers. In the substrate layer, it consists of a data center network in which physical servers are connected with physical links by physical switches/routers. A VDC embedding process maps requested virtual nodes and links onto physical nodes and paths of a data center network, respectively. Note that a VDC embedding process is a type of discussed VNE embedding processes, in which the substrate network is a data center network.

Traditional data centers are moving toward virtualized data centers in order to address their limitations regarding the network performance, security, and manageability [16]. Accordingly, in this chapter, we consider MapReduce-based virtual networks in VDCs. Each virtual network is heterogeneous, where a virtual node might be a mapper, a reducer, or a shuffler in a MapReduce framework. The data center network is also heterogeneous, with a multi-level topology such as a tree or a fat-tree. A substrate node could be a server, or a switch/router. In this case, a VDC embedding process requires to split computation-based virtual nodes (mappers/reducers) and embed them onto multiple physical servers, in order to parallelize the computation tasks. Nevertheless, all the existing embedding methods for virtual networks, e.g. [16, 29, 32, 38, 60, 62, 64, 69, 98,99] assume that a virtual node could be mapped only onto a single substrate node. Moreover, none of them provides a tool that could handle the heterogeneity of MapReduce-based VNs and the data center network. Therefore, they are not able to map the heterogeneous MapReduce-based VNs onto the heterogeneous data center network.

Incast traffic pattern in a data center network firstly introduced in [100]. In a MapReduce framework, multiple mappers may simultaneously send intermediate key and value pairs to a single reducer. Therefore, the bottleneck physical link over the physical path to the reducer will likely be congested. In non-virtualized data centers, the incast congestion normally happens in the bottleneck physical link that connects a shallow-buffered Top-of-Rack (ToR) switch to an end-server that hosts a reducer [101-105]. The converged traffic flows deplete either the switch's memory or the maximum allowed for that interface, resulting in packet loss [102]. The incast traffic also may cause a long queuing delay called incast queueing delay [102]. MapReduce requirements for low latency are directly related to the quality of the result returned and thus the revenue. Consequently, it is vital for the providers to control the incast delay. 
The incast problem in VDCs is different from the incast problem in non-virtualized (traditional) data centers. During a VDC embedding process, a specific amount of bandwidth capacity is allocated to each virtual link in substrate paths. The traffic flows in the allocated paths are limited to their assigned bandwidth capacity. Hence, the incast problem might happen over the bottleneck allocated paths to virtual links. In this case, the incast traffic may face the incast queuing delay in multiple physical links over the bottleneck allocated path, leading to a longer incast queuing delay in the virtual link. This problem is significantly different from the scenario when incast happens only in a single bottleneck physical link, which connects a ToR switch to an end-server, in the case of non-virtualized data centers. To the best of our knowledge this problem is not introduced in any existing research studies.

The existing research studies on the incast problem, for non-virtualized networks, are focused on creating new traffic control mechanisms, and updating Transmission Control Protocol (TCP). They either tried to decrease the packet loss by increasing the memory of shallow-buffered switches [102,106,107], or increase the recovery speed of TCP by decreasing the value of Retransmission Timeout (RTO) [104, 106, 108]. Nonetheless, the existing solutions for the incast problem are not efficient, and traffic control algorithms alone are difficult to mitigate the incast congestion [105]. Besides, they do not provide a solution for the incast problem in VDCs.

In this chapter, we propose GreenMap, an energy-efficient embedding method for MapReduce-based virtual networks that also controls the incast queuing delay. We formulate a Mixed Integer Disciplined Convex Program (MIDCP) for this problem. Since the formulated MIDCP is $\mathcal{N} \mathcal{P}$-hard, it is not scalable to large network sizes. Therefore, we also propose a novel and scalable heuristic for GreenMap. Both of the MIDCP and the heuristic map heterogeneous MapReduce-based VNs onto the heterogeneous data center network. They minimize a VDC's total consumed energy by physical servers, physical switches/routers, and physical links. They also control the incast queuing delay.

In this regard, we may split computation-based virtual nodes and map them onto multiple server substrate nodes. Accordingly, we address the emerged challenge of splitting and mapping adjacent virtual links of splitted and mapped virtual nodes. We might also collocate multiple splitted computation-based virtual nodes of a VN in a single server substrate node. The shuffler virtual nodes are assumed to be in the core/aggregation level in a data center network with a multi-level topology. Besides, 
shuffler virtual nodes do not perform large computation tasks, and therefore there is no need of parallel computations in this case. Thus, we do not split a shuffler virtual node. A shuffler virtual node is mapped only onto a single switch/router substrate node. But, we may collocate multiple shuffler virtual nodes of a VN in a single switch/router substrate node. Note that the substrate path that connects an allocated computation-based virtual node in a server substrate node to an allocated shuffler virtual node in a switch/router substrate node, or vice versa, may traverse through multiple relay switches/routers.

Our method also controls the introduced incast queueing delay in any adjacent virtual link of a mapped reducer virtual node. This is achieved by calculating the average end-to-end queueing delay for the incast traffic pattern during the virtual link mapping process. The incast queueing delay in an adjacent virtual link of an embedded reducer virtual node is related to the mentioned challenge of virtual link mapping. We demonstrate how controlling the incast queueing delay impacts the embedding process, the level of energy-saving, and the network's admittance ratio.

We evaluated both the MIDCP and the heuristic for GreenMap over randomly generated VDC scenarios. Simulation results prove the MIDCP and the heuristic save larger amounts of energy in the data center network than an existing energy-efficient embedding method for VNs that do not allow virtual node splitting. Besides, the simulations confirm that the incast queueing delay is controlled, and illustrate the influence of controlling the incast queueing delay on the energy-saving rates and the network's admittance ratio. It is also demonstrated that the heuristic could achieve closely to the optimum points set by the MIDCP.

In this regard, our contributions in this chapter are:

- The problem is formulated as an MIDCP.

- A novel and scalable heuristic is also proposed for the problem that can achieve closely to the optimum points.

- Different from [16,29,32,38,60,62,64,69,98,99], our approach makes it probable to split and map computation-based virtual nodes onto the data center network. Accordingly, it enables the providers to embed computation-based VNs onto a data center network.

- Different from [16, 29,32, 38,60,62, 64, 69, 98, 99], our solution handles the heterogeneity of MapReduce-based VNs and the data center network. 
- For the first time, we introduce a new incast problem for virtualized data centers.

- We demonstrate a novel approach that controls the introduced incast queueing delay.

- We tackle the incast problem during the provisioning process. So, it prevents the incast problem from happening at the first point. This is a considerably more efficient approach than the existing solutions for the incast in [102,104,106, 106-108] that attempt to recover the connection after that the incast happens.

- We examine both the MIDCP and the heuristic through extensive simulations, and check impacts of different factors.

- We demonstrate how controlling the incast queueing delay may affect the energy-saving level and the network's admittance ratio.

- To the best of our knowledge, this is the first study on the energy-efficient embedding of MapReduce-based virtual networks onto a data center network.

The rest of this chapter is organized as follows: We define our network model in Section 5.2, and study related power models in Section 5.3. The MIDCP and the heuristic for GreenMap are formulated in Section 5.4, and Section 5.5, respectively. The performance of the solutions is evaluated in Section 5.6. This chapter concludes in Section 5.7.

\subsection{The Network Model}

We consider a heterogeneous data center network with a multi-level topology, such as a tree or a fat-tree, as the substrate network. It is modeled as a directed graph $G_{s}=\left(V_{s}, E_{s}\right)$. The directed graph provides a higher level of flexibility in regard to routing traffic flows. $V_{s}$ and $E_{s}$ denote the set of substrate nodes and substrate links, respectively. The $i$ th substrate node $v_{s}^{i}$ could be a server, or a switch/router. The $i$ th substrate node, which is a server, is represented by $\bar{v}_{s}^{i} . C_{c}\left(\bar{v}_{s}^{i}\right)$ is the Central Processing Unit (CPU) capacity of $\bar{v}_{s}^{i}$. $\bar{V}_{s}$ denotes the set of server substrate nodes. Moreover, the $i$ th substrate node, which is a switch/router, is represented by $\tilde{v}_{s}^{i}$. $C_{b}\left(\tilde{v}_{s}^{i}\right)$ is the switching capacity of $\tilde{v}_{s}^{i}$. $\tilde{V}_{s}$ denotes the set of switch/router substrate nodes. Therefore, $V_{s}=\left\{\bar{V}_{s} \cup \tilde{V}_{s}\right\}$. 
A substrate link $l_{s}^{i, j}$ in the data center network connects the $i$ th substrate node to the $j$ th substrate node. $C_{b}\left(l_{s}^{i, j}\right)$ is the bandwidth capacity of $l_{s}^{i, j}$.

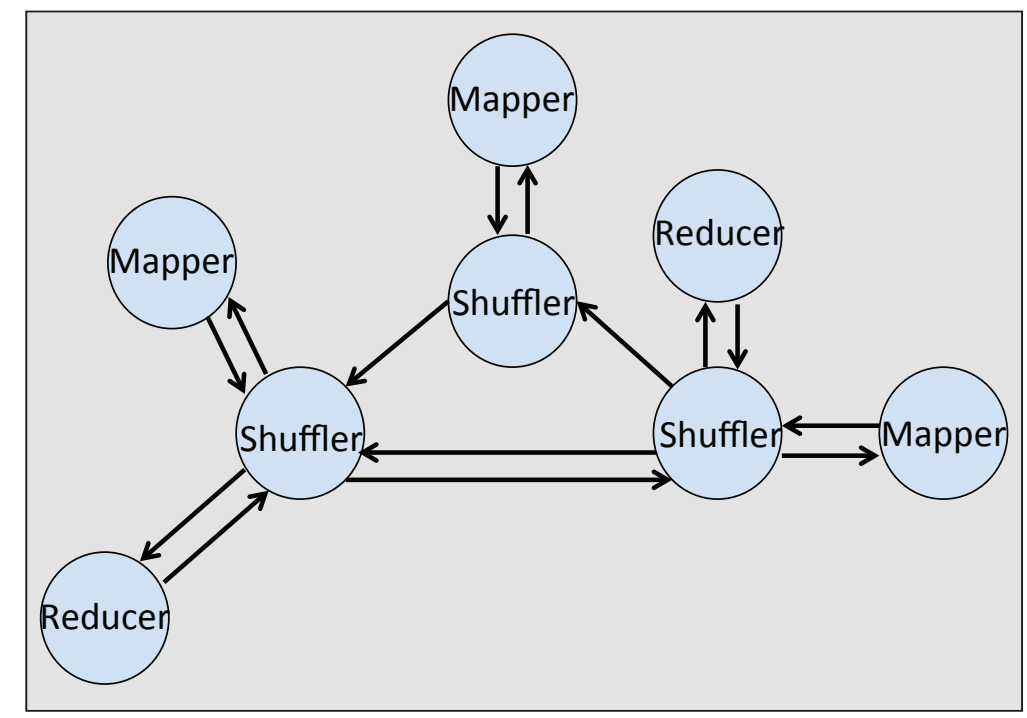

Figure 5.1: Example: A MapReduce-based VN's topology

The $n$th virtual network is modeled as a directed graph $G_{n}=\left(V_{n}, E_{n}\right)$, where $V_{n}$ and $E_{n}$ denote the set of virtual nodes and virtual links in the $n$th $\mathrm{VN}$, respectively. $\Phi$ is the set of all of the VNs, and $L_{n}=\left|E_{n}\right|$. Figure 5.1 shows an example of a MapReduce-based VN's topology. In this framework, the $k$ th virtual node $v_{n}^{k}$ in the $n$th VN could be a mapper, a reducer, or a shuffler. The $k$ th virtual node in the $n$th $\mathrm{VN}$, which is a mapper, is represented by $\dot{v}_{n}^{k} . \hat{C}_{c}\left(\dot{v}_{n}^{k}\right)$ is the requested CPU capacity for $\dot{v}_{n}^{k}$. VN customers may specify the minimum $\hat{C}_{c}^{\check{m}}\left(\dot{v}_{n}^{k}\right)$ and the maximum $\hat{C}_{c}^{\hat{m}}\left(\dot{v}_{n}^{k}\right)$ amount of required CPU capacity per allocated physical machine for $\dot{v}_{n}^{k}$. We assume that a mapper virtual node $\dot{v}_{n}^{k}$ generates traffic that follows Poisson process with the mean rate of $\lambda\left(\dot{v}_{n}^{k}\right)$. Considering $\dot{V}_{n}$ as the set of mapper virtual nodes in the $n$th $\mathrm{VN}, \lambda_{n}^{\mathcal{M}}$ is equal to $\sum_{k \in \dot{V}_{n}} \lambda\left(\dot{v}_{n}^{k}\right)$. Besides, the $k$ th virtual node in the $n$th $\mathrm{VN}$, which is a reducer, is represented by $\ddot{v}_{n}^{k}$. $\hat{C}_{c}\left(\ddot{v}_{n}^{k}\right)$ is the demanded CPU capacity for $\ddot{v}_{n}^{k}$. VN customers also may specify the minimum $\hat{C}_{c}^{\check{m}}\left(\ddot{v}_{n}^{k}\right)$ and the maximum $\hat{C}_{c}^{\hat{m}}\left(\ddot{v}_{n}^{k}\right)$ amount of required CPU capacity per allocated physical machine for $\ddot{v}_{n}^{k}$. $\ddot{V}_{n}$ denotes the set of reducer virtual nodes in the $n$th VN. Furthermore, the $k$ th virtual node in the $n$th $\mathrm{VN}$, which is a shuffler, is represented by $\tilde{v}_{n}^{k}$. We assume that a shuffler virtual node $\tilde{v}_{n}^{k}$ only has the switching capacity demand $\hat{C}_{b}\left(\tilde{v}_{n}^{k}\right)$, that is equal to the summation of its adjacent virtual links' bandwidth demands. $\tilde{V}_{n}$ denotes the set of shuffler virtual 
nodes in the $n$th VN. Therefore, $V_{n}=\left\{\dot{V}_{n} \cup \ddot{V}_{n} \cup \tilde{V}_{n}\right\}$.

The set of virtual links are presented as a set of ordered virtual node pairs $l_{n}^{a^{m}, b^{m}}, m=1,2, \ldots, L_{n}$. $a^{m}$ and $b^{m}$ are the source and the sink virtual nodes of the $m$ th virtual link in the corresponding VN, respectively. $\hat{C}_{b}\left(l_{n}^{a^{m}}, b^{m}\right)$ is the bandwidth demand of $l_{n}^{a^{m}, b^{m}}$. We presume computation-based virtual nodes are connected through shuffler virtual nodes in a virtual network topology. In other words, there is no pair of computation-based virtual nodes that are connected directly to each other in a VN topology. This is the real case in a MapReduce framework.

\subsection{Power Models}

Considering the heterogeneous data center network as the substrate network, we have three major power consumers, physical servers, physical switches/routers, and physical links. In this chapter, we intend to minimize the total consumed energy by them in a VDC. We study a power model for each of these substrate elements.

Equation 5.1 defines the actual power consumption $\tilde{p}\left(\bar{v}_{s}^{i}\right)$ for a server substrate node $\bar{v}_{s}^{i}$ [58]. $\alpha\left(\bar{v}_{s}^{i}\right)$ denotes the status of $\bar{v}_{s}^{i}$. It is 1 , if $\bar{v}_{s}^{i}$ is active. Otherwise, it is 0 . $\tilde{p}^{b}\left(\bar{v}_{s}^{i}\right)$ is the base power consumption of $\bar{v}_{s}^{i}$ required to keep it active. $\tilde{p}^{m}\left(\bar{v}_{s}^{i}\right)$ is the maximum power consumption of $\bar{v}_{s}^{i}$. The total allocated processing capacity $\check{\phi}\left(\bar{v}_{s}^{i}\right)$ to computation-based virtual nodes in $\bar{v}_{s}^{i}$ changes its actual power consumption between $\tilde{p}^{b}\left(\bar{v}_{s}^{i}\right)$ and $\tilde{p}^{m}\left(\bar{v}_{s}^{i}\right)$, linearly. $\tilde{p}^{b}\left(\bar{v}_{s}^{i}\right)$ and $\tilde{p}^{m}\left(\bar{v}_{s}^{i}\right)$ could be found through calibration experiments, e.g. in [59].

$$
\tilde{p}\left(\bar{v}_{s}^{i}\right)=\alpha\left(\bar{v}_{s}^{i}\right) \tilde{p}^{b}\left(\bar{v}_{s}^{i}\right)+\frac{\check{\phi}\left(\bar{v}_{s}^{i}\right)}{C_{c}\left(\bar{v}_{s}^{i}\right)}\left(\tilde{p}^{m}\left(\bar{v}_{s}^{i}\right)-\tilde{p}^{b}\left(\bar{v}_{s}^{i}\right)\right)
$$

Equation 5.2 defines the actual power consumption $\tilde{p}\left(\tilde{v}_{s}^{i}\right)$ for a switch/router substrate node $\tilde{v}_{s}^{i}[56] . \quad \alpha\left(\tilde{v}_{s}^{i}\right)$ shows the status of $\tilde{v}_{s}^{i} \cdot \tilde{p}^{b}\left(\tilde{v}_{s}^{i}\right)$ and $\tilde{p}^{m}\left(\tilde{v}_{s}^{i}\right)$ are the base and the maximum power consumption of $\tilde{v}_{s}^{i}$, respectively. The total traffic load $r\left(\tilde{v}_{s}^{i}\right)$ in $\tilde{v}_{s}^{i}$ changes its actual power consumption between its base and maximum power consumption, linearly.

$$
\tilde{p}\left(\tilde{v}_{s}^{i}\right)=\alpha\left(\tilde{v}_{s}^{i}\right) \tilde{p}^{b}\left(\tilde{v}_{s}^{i}\right)+\frac{r\left(\tilde{v}_{s}^{i}\right)}{C_{b}\left(\tilde{v}_{s}^{i}\right)}\left(\tilde{p}^{m}\left(\tilde{v}_{s}^{i}\right)-\tilde{p}^{b}\left(\tilde{v}_{s}^{i}\right)\right)
$$

According to [56], $\tilde{p}^{b}\left(\tilde{v}_{s}^{i}\right)$ is $0.85 C_{b}\left(\tilde{v}_{s}^{i}\right)^{\frac{2}{3}}$, and $\tilde{p}^{m}\left(\tilde{v}_{s}^{i}\right)$ is $C_{b}\left(\tilde{v}_{s}^{i}\right)^{\frac{2}{3}}$. Consequently, 
Equation 5.2 is rewritten in Equation 5.3.

$$
\tilde{p}\left(\tilde{v}_{s}^{i}\right)=0.85 \alpha\left(\tilde{v}_{s}^{i}\right) C_{b}\left(\tilde{v}_{s}^{i}\right)^{\frac{2}{3}}+\frac{0.15 r\left(\tilde{v}_{s}^{i}\right)}{C_{b}\left(\tilde{v}_{s}^{i}\right)^{\frac{1}{3}}}
$$

Similarly, Equation 5.4 defines the actual power consumption $\tilde{p}\left(l_{s}^{i, j}\right)$ of a substrate link $l_{s}^{i, j}[56] . \tilde{p}^{b}\left(l_{s}^{i, j}\right)$ and $\tilde{p}^{m}\left(l_{s}^{i, j}\right)$ are the base and the maximum power consumption of $l_{s}^{i, j}$, respectively. The total allocated traffic capacity $r\left(l_{s}^{i, j}\right)$ to virtual links in $l_{s}^{i, j}$ varies its actual power consumption, linearly. Note that $\tilde{p}^{b}\left(l_{s}^{i, j}\right)$ and $\tilde{p}^{m}\left(l_{s}^{i, j}\right)$ are normally defined for different ranges of link bandwidth capacity, based on the link's length and the type of the cable. Some numerical amounts for them are given in [56].

$$
\tilde{p}\left(l_{s}^{i, j}\right)=\alpha\left(l_{s}^{i, j}\right) \tilde{p}^{b}\left(l_{s}^{i, j}\right)+\frac{r\left(l_{s}^{i, j}\right)}{C_{b}\left(l_{s}^{i, j}\right)}\left(\tilde{p}^{m}\left(l_{s}^{i, j}\right)-\tilde{p}^{b}\left(l_{s}^{i, j}\right)\right)
$$

According to the above defined power models, the most effective way of saving energy in any of the mentioned substrate elements is shutting down the device. Note that the same amount of processing/traffic demand might cause different amounts of power consumption in distinct substrate elements, based on the element's processing/bandwidth capacity, its base power consumption, and its maximum power consumption.

\subsection{The Mixed Integer Disciplined Convex Pro- gram}

In this problem, the processing/bandwidth capacity of every substrate node, and the bandwidth capacity of every substrate link are given. Besides, each VN's topology, the processing/bandwidth demand of every virtual node and their minimum/maximum processing demands per physical server, the bandwidth demand of every virtual link, and $\lambda\left(\dot{v}_{n}^{k}\right)$ of each mapper virtual node are known. We need to find a mapping for every VN such that the data center network's total energy consumption by physical servers, physical switches/routers, and physical links, is minimized. We also require to control the incast queuing delay according to the given $\hat{D}$, which is the maximum tolerable queuing delay in a virtual link. 
As it is discussed, we may split the computation-based virtual nodes and embed them onto multiple physical servers. But, we map a shuffler virtual node onto a single physical switch/router. We also may collocate virtual nodes (mappers/reducers/shufflers) of a VN in a single relevant substrate node. A new challenge will emerge in this model. The issue is what is the bandwidth capacity that needs to be allocated to an adjacent virtual link of a splitted and mapped computation-based virtual node. Likely, a splitted computation-based virtual node processes proportional traffic to its assigned processing capacity. Therefore, the amount of bandwidth we allocate to an adjacent virtual link of a splitted and mapped computation-based virtual node is proportional to its assigned processing capacity. Thus, in this model, more likely the introduced incast problem may arise in an adjacent virtual link of a splitted reducer virtual node. Note that in order to avoid out of order packet delivery, we do not split generated traffic of an allocated virtual node.

In order to control the introduced incast queueing delay, it is required to find the end-to-end queuing delay for the incast traffic pattern in the substrate path allocated to every virtual link terminates at a splitted and mapped reducer virtual node. In the incast traffic pattern, $\lambda_{n}^{\mathcal{M}}$ is the mean traffic rate in the substrate path. The majority of today's switches/routers are internally non-blocking (i.e. the internal switch fabric speed is considerably faster than each output port). Therefore, traffic can only be blocked by limited bandwidths of output ports which are defined earlier by the link bandwidth capacity. We model the queue of an allocated bandwidth capacity to a virtual link in a substrate link by $\mathrm{M} / \mathrm{M} / 1$ queue. According to Jackson Networks theorem and because we do not split generated traffic of an allocated virtual node, the end-to-end incast queuing delay in the substrate path could be calculated by knowing the amount of allocated traffic capacity to the virtual link in each physical link over the substrate path, and $\lambda_{n}^{\mathcal{M}}$. Since the amount of bandwidth we allocate to the virtual links are proportional to the assigned capacity of its end virtual nodes, the way we split reducer virtual nodes directly impacts the incast queueing delay. Besides, the substrate node which we map the splitted reducer virtual node onto, and accordingly the allocated substrate path to each of the adjacent virtual links, also may influence the incast queuing delay. Clearly, this limits the level of freedom regarding the energy-efficient embedding of the VNs, and may affect the energy-saving rate and the network's admittance ratio.

Considering the discussed definitions, we formulate the following MIDCP as a 
solution for GreenMap:

\section{Optimization Variables:}

- $\check{\phi}\left(v_{n}^{k}, v_{s}^{i}\right)$ is a real variable. It represents a fraction of processing/switching demand of a virtual node $v_{n}^{k}$, that is allocated in a substrate node $v_{s}^{i}$. $v_{n}^{k}$ could be $\dot{v}_{n}^{k}, \ddot{v}_{n}^{k}$, or $\tilde{v}_{n}^{k}$. $v_{s}^{i}$ could be $\bar{v}_{s}^{i}$ or $\tilde{v}_{s}^{i}$.

- $\alpha\left(\bar{v}_{s}^{i}\right), \alpha\left(\tilde{v}_{s}^{i}\right)$, and $\alpha\left(l_{s}^{i, j}\right)$ are binary variables. They denote the status of respective substrate node/link. The variable is 1 in the case the device is active. Otherwise, it is 0 .

- $\alpha\left(\dot{v}_{n}^{k}, \bar{v}_{s}^{i}\right), \alpha\left(\ddot{v}_{n}^{k}, \bar{v}_{s}^{i}\right), \alpha\left(\tilde{v}_{n}^{k}, \tilde{v}_{s}^{i}\right)$ are binary variables denote whether a virtual node is allocated in a substrate node (the variable is 1 ), or not (the variable is 0 ).

- $\alpha\left(l_{n}^{x, y}(m)\right)$ is a binary variable. It is 1 , if the whole or a fraction of $v_{n}^{a^{m}}$ 's processing/switching demand is allocated in $v_{s}^{x}$, and the whole or a fraction of $v_{n}^{b^{m}}$ 's processing/switching demand is allocated in $v_{s}^{y}$. Otherwise, it is $0 . l_{n}^{x, y}(m)$ is a sub virtual link of $l_{n}^{a^{m}, b^{m}}$ that connects the allocated source virtual node in $v_{s}^{x}$ to the allocated sink virtual node in $v_{s}^{y}$.

- $z^{i, j}\left(l_{n}^{x, y}(m)\right)$ is a binary variable. It is 1 if the allocated substrate path for $l_{n}^{x, y}(m)$ passes through $l_{s}^{i, j}$. Otherwise, it is 0 .

- $\dot{d}_{n}^{x, y}(m)$ is a real variable. It is a fraction of $\hat{C}_{b}\left(l_{n}^{a^{m}, b^{m}}\right)$ that needs to be allocated to $l_{n}^{x, y}(m)$.

- $\ddot{d}^{i, j}\left(l_{n}^{x, y}(m)\right)$ is a real variable. It shows the amount of allocated traffic capacity to $l_{n}^{x, y}(m)$ in $l_{s}^{i, j}$.

Objective Function: Our objective is minimizing the total consumed energy by physical servers, physical switches/routers, and physical links, in a VDC. Equation 5.5 maintains this objective.

$$
\begin{array}{r}
\text { Minimize }\left\{\sum_{i \in \bar{V}_{s}} \alpha\left(\bar{v}_{s}^{i}\right) \tilde{p}^{b}\left(\bar{v}_{s}^{i}\right)+\frac{\check{\phi}\left(\bar{v}_{s}^{i}\right)}{C_{c}\left(\bar{v}_{s}^{i}\right)}\left(\tilde{p}^{m}\left(\bar{v}_{s}^{i}\right)-\tilde{p}^{b}\left(\bar{v}_{s}^{i}\right)\right)\right. \\
+\sum_{i \in \tilde{V}_{s}} 0.85 \alpha\left(\tilde{v}_{s}^{i}\right) C_{b}\left(\tilde{v}_{s}^{i}\right)^{\frac{2}{3}}+\frac{0.15 r\left(\tilde{v}_{s}^{i}\right)}{C_{b}\left(\tilde{v}_{s}^{i}\right)^{\frac{1}{3}}} \\
\left.+\sum_{(i, j) \in E_{s}} \alpha\left(l_{s}^{i, j}\right) \tilde{p}^{b}\left(l_{s}^{i, j}\right)+\frac{r\left(l_{s}^{i, j}\right)}{C_{b}\left(l_{s}^{i, j}\right)}\left(\tilde{p}^{m}\left(l_{s}^{i, j}\right)-\tilde{p}^{b}\left(l_{s}^{i, j}\right)\right)\right\}
\end{array}
$$


Constraints: Every virtual node must be mapped onto one or multiple substrate nodes. This is ensured by the first constraint in Equation 5.6. Note that this constraint allows collocation of multiple virtual nodes of a $\mathrm{VN}$ onto a single substrate node. The constraint in Equation 5.7 prevents a computation-based virtual node from being mapped onto a switch/router substrate node. Besides, the constraint in Equation 5.8 forbids a shuffler virtual node to be mapped onto a server substrate node.

$$
\begin{gathered}
\sum_{i \in V_{s}} \check{\phi}\left(v_{n}^{k}, v_{s}^{i}\right)=1, \quad \forall n \in\left\{n \mid G_{n} \in \Phi\right\}, \forall k \in V_{n} \\
\sum_{n \in\left\{n \mid G_{n} \in \Phi\right\}}\left(\sum_{k \in \dot{V}_{n}} \check{\phi}\left(\dot{v}_{n}^{k}, \tilde{v}_{s}^{i}\right)+\sum_{k \in \ddot{V}_{n}} \check{\phi}\left(\ddot{v}_{n}^{k}, \tilde{v}_{s}^{i}\right)\right)=0, \quad \forall i \in \tilde{V}_{s} \\
\sum_{n \in\left\{n \mid G_{n} \in \Phi\right\}} \sum_{k \in \tilde{V}_{n}} \check{\phi}\left(\tilde{v}_{n}^{k}, \bar{v}_{s}^{i}\right)=0, \quad \forall i \in \bar{V}_{s}
\end{gathered}
$$

The allocated processing capacity to a computation-based virtual node in a server substrate node must be equal or greater than the requested minimum CPU capacity per physical machine for the virtual node, and equal or less than the requested maximum CPU capacity per physical machine for the virtual node. This is confirmed in the constraint in Equation 5.9 for mapper virtual nodes, and in the constraint in Equation 5.10 for reducer virtual nodes. Note that the given ratio must be feasible. For example, $\frac{\hat{C}_{c}^{\check{m}}\left(\ddot{v}_{n}^{k}\right)}{\hat{C}_{c}\left(\ddot{v}_{n}^{k}\right)}$ can not be greater than 0.5 .

$$
\begin{aligned}
& \frac{\hat{C}_{c}^{\check{m}}\left(\dot{v}_{n}^{k}\right)}{\hat{C}_{c}\left(\dot{v}_{n}^{k}\right)} \alpha\left(\dot{v}_{n}^{k}, \bar{v}_{s}^{i}\right) \leq \check{\phi}\left(\dot{v}_{n}^{k}, \bar{v}_{s}^{i}\right) \leq \frac{\hat{C}_{c}^{\hat{m}}\left(\dot{v}_{n}^{k}\right)}{\hat{C}_{c}\left(\dot{v}_{n}^{k}\right)} \alpha\left(\dot{v}_{n}^{k}, \bar{v}_{s}^{i}\right), \\
& \forall i \in \bar{V}_{s}, \forall n \in\left\{n \mid G_{n} \in \Phi\right\}, \forall k \in \dot{V}_{n} \\
& \frac{\hat{C}_{c}^{m}\left(\ddot{v}_{n}^{k}\right)}{\hat{C}_{c}\left(v_{n}^{k}\right)} \alpha\left(\ddot{v}_{n}^{k}, \bar{v}_{s}^{i}\right) \leq \check{\phi}\left(\ddot{v}_{n}^{k}, \bar{v}_{s}^{i}\right) \leq \frac{\hat{C}_{c}^{\hat{m}}\left(\ddot{v}_{n}^{k}\right)}{\hat{C}_{c}\left(\ddot{v}_{n}^{k}\right)} \alpha\left(\ddot{v}_{n}^{k}, \bar{v}_{s}^{i}\right), \\
& \forall i \in \bar{V}_{s}, \forall n \in\left\{n \mid G_{n} \in \Phi\right\}, \forall k \in \ddot{V}_{n}
\end{aligned}
$$

The constraint in Equation 5.11 restricts the program to map a shuffler virtual node only onto one switch/router substrate node.

$$
\check{\phi}\left(\tilde{v}_{n}^{k}, \tilde{v}_{s}^{i}\right)=\alpha\left(\tilde{v}_{n}^{k}, \tilde{v}_{s}^{i}\right), \quad \forall i \in \tilde{V}_{s}, \forall n \in\left\{n \mid G_{n} \in \Phi\right\}, \forall k \in \tilde{V}_{n}
$$


Besides, the total allocated processing capacity to computation-based virtual nodes in a server substrate node must be equal or less than the substrate node's CPU capacity, as shown in the constraint in Equation 5.12. The total allocated switching capacity to shuffler virtual nodes in a switch/router substrate node also must be equal or less than the substrate node's switching capacity, as indicated in the constraint in Equation 5.13.

$$
\begin{gathered}
\sum_{n \in\left\{n \mid G_{n} \in \Phi\right\}}\left(\sum_{k \in \dot{V}_{n}} \check{\phi}\left(\dot{v}_{n}^{k}, \bar{v}_{s}^{i}\right) \hat{C}_{c}\left(\dot{v}_{n}^{k}\right)+\sum_{k \in \ddot{V}_{n}} \check{\phi}\left(\ddot{v}_{n}^{k}, \bar{v}_{s}^{i}\right) \hat{C}_{c}\left(\ddot{v}_{n}^{k}\right)\right) \leq C_{c}\left(\bar{v}_{s}^{i}\right), \quad \forall i \in \bar{V}_{s} \\
\sum_{n \in\left\{n \mid G_{n} \in \Phi\right\}} \sum_{k \in \tilde{V}_{n}} \check{\phi}\left(\tilde{v}_{n}^{k}, \tilde{v}_{s}^{i}\right) \hat{C}_{b}\left(\tilde{v}_{n}^{k}\right) \leq C_{b}\left(\tilde{v}_{s}^{i}\right), \quad \forall i \in \tilde{V}_{s}
\end{gathered}
$$

In the next step, the program needs to map the adjacent virtual links of the allocated virtual nodes. We discussed that computation-based virtual nodes are connected through shuffler virtual nodes in a VN topology. So, we have two types of virtual links in a VN. First, a virtual link that connects a computation-based virtual node to a shuffler virtual node, or vice versa. Second, a virtual link that connects a shuffler virtual node to another shuffler virtual node. Therefore, $\check{\phi}\left(v_{n}^{a^{m}}, v_{s}^{x}\right) \check{\phi}\left(v_{n}^{b^{m}}, v_{s}^{y}\right) \hat{C}_{b}\left(l_{n}^{a^{m}, b^{m}}\right)$ is the fraction of $\hat{C}_{b}\left(l_{n}^{a^{m}, b^{m}}\right)$ that needs to be allocated to $l_{n}^{x, y}(m)$. This amount is proportional to the assigned processing/switching capacity to the mapped source and sink virtual nodes of the virtual link $l_{n}^{a^{m}, b^{m}}$. Accordingly, the linear constraints in Equation 5.14 force $\dot{d}_{n}^{x, y}(m)$ to take the value of $\check{\phi}\left(v_{n}^{a^{m}}, v_{s}^{x}\right) \check{\phi}\left(v_{n}^{b^{m}}, v_{s}^{y}\right)$.

$$
\begin{gathered}
\dot{d}_{n}^{x, y}(m) \leq \check{\phi}\left(v_{n}^{a^{m}}, v_{s}^{x}\right), \\
\dot{d}_{n}^{x, y}(m) \leq \check{\phi}\left(v_{n}^{b^{m}}, v_{s}^{y}\right), \\
\dot{d}_{n}^{x, y}(m) \geq \check{\phi}\left(v_{n}^{a^{m}}, v_{s}^{x}\right)+\check{\phi}\left(v_{n}^{b_{m}}, v_{s}^{y}\right)-1, \\
\dot{d}_{n}^{x, y}(m) \geq 0, \\
\forall x \in V_{s}, \forall y \in V_{s}, \forall n \in\left\{n \mid G_{n} \in \Phi\right\}, m=1, \ldots, L_{n}
\end{gathered}
$$

$B_{1}, B_{2}, B_{3}$, and $B_{4}$ are large integer numbers. They must be large enough to be greater than the largest amount of the left-hand side of their respective inequality. If there is a non-zero bandwidth demand for $l_{n}^{x, y}(m)$, then the first constraint in Equation 5.15 forces $\alpha\left(l_{n}^{x, y}(m)\right)$ to be 1. Then, the second constraint in Equation 5.15 needs to route a single unit of data from the $x$ th substrate node to the $y$ th substrate node. Because 
the variable $z^{i, j}\left(l_{n}^{x, y}(m)\right)$ is binary, the unit of data could not be splitted. Besides, the third constraint in Equation 5.15 limits the program routing, so the maximum number of incoming and outgoing flows for a sub virtual link, in any substrate node, is two. This maintains a single loopless path. The driven route will be used as the substrate path for $l_{n}^{x, y}(m)$. Note that if $\alpha\left(l_{n}^{x, y}(m)\right)=0$, then no substrate path is allocated to $l_{n}^{x, y}(m)$.

$$
\begin{aligned}
& \dot{d}_{n}^{x, y}(m) \hat{C}_{b}\left(l_{n}^{a^{m}, b^{m}}\right) \leq B_{1} \alpha\left(l_{n}^{x, y}(m)\right), \\
& \sum_{\left\{j \mid(i, j) \in E_{s}\right\}} z^{i, j}\left(l_{n}^{x, y}(m)\right)-\sum_{\left\{j \mid(j, i) \in E_{s}\right\}} z^{j, i}\left(l_{n}^{x, y}(m)\right)= \begin{cases}\alpha\left(l_{n}^{x, y}(m)\right) & \text { if } \quad i=x \\
-\alpha\left(l_{n}^{x, y}(m)\right) & \text { if } i=y \\
0 & \text { otherwise }\end{cases} \\
& \sum_{\left\{j \mid(i, j) \in E_{s}\right\}} z^{i, j}\left(l_{n}^{x, y}(m)\right)+\sum_{\left\{j \mid(j, i) \in E_{s}\right\}} z^{j, i}\left(l_{n}^{x, y}(m)\right) \leq 2, \\
& \forall i \in V_{s}, \forall x \in V_{s}, \forall y \in V_{s}, \forall n \in\left\{n \mid G_{n} \in \Phi\right\}, m=1, \ldots, L_{n}
\end{aligned}
$$

Furthermore, the total allocated traffic capacity $r\left(l_{s}^{i, j}\right)$ in a substrate link $l_{s}^{i, j}$ must be less than its physical bandwidth capacity $C_{b}\left(l_{s}^{i, j}\right)$, as expressed in the constraint in Equation 5.16. $r\left(l_{s}^{i, j}\right)$ is the summation of every allocated traffic capacity $\ddot{d}^{i, j}\left(l_{n}^{x, y}(m)\right)$ to a sub virtual link $l_{n}^{x, y}(m)$ in $l_{s}^{i, j} . \ddot{d}^{i, j}\left(l_{n}^{x, y}(m)\right)$ is equal to $z^{i, j}\left(l_{n}^{x, y}(m)\right) \dot{d}_{n}^{x, y}(m) \hat{C}_{b}\left(l_{n}^{a^{m}, b^{m}}\right)$. If the allocated substrate path to $l_{n}^{x, y}(m)$ passes through $l_{s}^{i, j}$, then $z^{i, j}\left(l_{n}^{x, y}(m)\right)$ is 1 , and therefore $\ddot{d}^{i, j}\left(l_{n}^{x, y}(m)\right)$ is $\dot{d}_{n}^{x, y}(m) \hat{C}_{b}\left(l_{n}^{a^{m}, b^{m}}\right)$. Otherwise, $\ddot{d}^{i, j}\left(l_{n}^{x, y}(m)\right)$ is 0. The linear constraints in Equation 5.17 force $\ddot{d}^{i, j}\left(l_{n}^{x, y}(m)\right)$ to take the value of $z^{i, j}\left(l_{n}^{x, y}(m)\right) \dot{d}_{n}^{x, y}(m) \hat{C}_{b}\left(l_{n}^{a^{m}, b^{m}}\right)$. Note that $M$ is the largest virtual link bandwidth demand in $\Phi$.

$$
\begin{gathered}
r\left(l_{s}^{i, j}\right) \leq C_{b}\left(l_{s}^{i, j}\right), \quad \forall(i, j) \in E_{s}, \quad \text { where: } \\
r\left(l_{s}^{i, j}\right)=\sum_{x \in V_{s}} \sum_{y \in V_{s}} \sum_{n \in\left\{n \mid G_{n} \in \Phi\right\}} \sum_{m=1}^{L_{n}} \ddot{d}^{i, j}\left(l_{n}^{x, y}(m)\right) \\
\dddot{d}^{i, j}\left(l_{n}^{x, y}(m)\right) \leq M z^{i, j}\left(l_{n}^{x, y}(m)\right),
\end{gathered}
$$




$$
\begin{gathered}
\ddot{d}^{i, j}\left(l_{n}^{x, y}(m)\right) \leq \dot{d}_{n}^{x, y}(m) \hat{C}_{b}\left(l_{n}^{a^{m}, b^{m}}\right), \\
\dddot{d}^{i, j}\left(l_{n}^{x, y}(m)\right) \geq \dot{d}_{n}^{x, y}(m) \hat{C}_{b}\left(l_{n}^{a^{m}, b^{m}}\right)-M\left(1-z^{i, j}\left(l_{n}^{x, y}(m)\right)\right), \\
\ddot{d}^{i, j}\left(l_{n}^{x, y}(m)\right) \geq 0, \\
\forall(i, j) \in E_{s}, \forall x \in V_{s}, \forall y \in V_{s}, \forall n \in\left\{n \mid G_{n} \in \Phi\right\}, m=1, \ldots, L_{n}
\end{gathered}
$$

Moreover, the total incoming and outgoing traffic of a switch/router substrate node must be less than its switching capacity. This is confirmed in the constraint in Equation 5.18.

$$
r\left(\tilde{v}_{s}^{i}\right) \leq C_{b}\left(\tilde{v}_{s}^{i}\right), \forall i \in \tilde{V}_{s}, \quad \text { where: }
$$

$$
r\left(\tilde{v}_{s}^{i}\right)=\sum_{(i, j) \in E_{s}} r\left(l_{s}^{i, j}\right)+\sum_{(j, i) \in E_{s}} r\left(l_{s}^{j, i}\right)
$$

Considering M/M/1 queue and Jackson Networks theorem, and because we do not split generated traffic by an allocated virtual node, the average end-to-end incast queuing delay of a sub virtual link $l_{n}^{x, y}(m)$ could be calculated by the sum of $\frac{1}{\mu\left(l_{n}^{x, y}(m)\right)-\lambda_{n}^{\mathcal{M}}}$ for every substrate link $l_{s}^{i, j}$ that a traffic capacity is allocated to $l_{n}^{x, y}(m)$ in it $\left(z^{i, j}\left(l_{n}^{x, y}(m)\right)=1\right)$. The mean service rate $\mu\left(l_{n}^{x, y}(m)\right)$ for $l_{n}^{x, y}(m)$ is equal to $\dot{d}_{n}^{x, y}(m) \hat{C}_{b}\left(l_{n}^{a^{m}, b^{m}}\right)$.

Considering the continuous function $f(g, h)=\frac{g^{2}}{h}, h>0$, the hessian matrix $H$ of $f$ is shown in Equation 5.19. Eigenvalues of $H$ are 0 and $\frac{2 g^{2}+2 h^{2}}{h^{3}}$. Because all eigenvalues of $H$ are non-negative, $H$ is positive semi-definite. Therefore, $f$ is jointly convex on both $g$ and $h$.

$$
H=\left[\begin{array}{cc}
\frac{2}{h} & \frac{-2 g}{h^{2}} \\
\frac{-2 g}{h^{2}} & \frac{2 g^{2}}{h^{3}}
\end{array}\right]
$$

Thus, the left-hand side of the first inequality constraint in Equation 5.20 calculates the end-to-end incast queueing delay in the allocated substrate path to a sub virtual link that connects a mapped shuffler virtual node to a mapped and splitted reducer virtual node. Note that if no traffic capacity is allocated to a sub virtual link $l_{n}^{x, y}(m)$ in a substrate link $l_{s}^{i, j}$, then $z^{i, j}\left(l_{n}^{x, y}(m)\right)$ is 0 , and therefore the delay is 0 . $2(1-$ $\left.\alpha\left(l_{n}^{x, y}(m)\right)\right)$ is added to ensure the denominator is never 0 . According to the first constraint in Equation 5.15, if $\dot{d}_{n}^{x, y}(m)$ is greater than 0, then $\alpha\left(l_{n}^{x, y}(m)\right)$ is 1 , and therefore the denominator is $\dot{d}_{n}^{x, y}(m) \hat{C}_{b}\left(l_{n}^{a^{m}, b^{m}}\right)-\lambda_{n}^{\mathcal{M}}$. Otherwise, if $\dot{d}_{n}^{x, y}(m)$ is 0 , then 
$z^{i, j}\left(l_{n}^{x, y}(m)\right)$ of any substrate link is 0 , and the denominator is $\lambda_{n}^{\mathcal{M}}$.

Hence, the first inequality constraint in Equation 5.20 is a mixed integer disciplined convex constraint [109], which verifies the incast queueing delay in the allocated substrate path to every virtual link that terminates at a mapped reducer virtual node, is less than $\hat{D}$. Besides, the second constraint in Equation 5.20 confirms that every queue in the network is stable.

$$
\begin{gathered}
\sum_{(i, j) \in E_{s}} \frac{z^{i, j}\left(l_{n}^{x, y}(m)\right)^{2}}{\dot{d}_{n}^{x, y}(m) \hat{C}_{b}\left(l_{n}^{a^{m}, b^{m}}\right)-\lambda_{n}^{\mathcal{M}}+2\left(1-\alpha\left(l_{n}^{x, y}(m)\right)\right) \lambda_{n}^{\mathcal{M}}} \leq \hat{D}, \\
\dot{d}_{n}^{x, y}(m) \hat{C}_{b}\left(l_{n}^{a^{m}, b^{m}}\right)>\alpha\left(l_{n}^{x, y}(m)\right) \lambda_{n}^{\mathcal{M}}, \\
\forall n \in\left\{n \mid G_{n} \in \Phi\right\}, m \in\left\{m \mid m=1, \ldots, L_{n} ; b^{m} \in \ddot{V}_{n}\right\}, \forall x \in V_{s}, \forall y \in V_{s}
\end{gathered}
$$

The constraints in Equation 5.21 check the respective device's status.

$$
\begin{gathered}
\check{\phi}\left(\bar{v}_{s}^{i}\right) \leq B_{2} \alpha\left(\bar{v}_{s}^{i}\right), \forall i \in \bar{V}_{s}, \quad \text { where: } \\
\qquad \begin{aligned}
\check{\phi}\left(\bar{v}_{s}^{i}\right)=\sum_{n \in G_{n}}\left(\sum_{k \in \dot{V}_{n}} \check{\phi}\left(\dot{v}_{n}^{k}, \bar{v}_{s}^{i}\right) \hat{C}_{c}\left(\dot{v}_{n}^{k}\right)+\sum_{k \in \ddot{V}_{n}} \check{\phi}\left(\ddot{v}_{n}^{k}, \bar{v}_{s}^{i}\right) \hat{C}_{c}\left(\ddot{v}_{n}^{k}\right)\right) ; \\
r\left(\tilde{v}_{s}^{i}\right) \leq B_{3} \alpha\left(\tilde{v}_{s}^{i}\right), \quad \forall i \in \tilde{V}_{s} ; \\
r\left(l_{s}^{i, j}\right) \leq B_{4} \alpha\left(l_{s}^{i, j}\right), \quad \forall(i, j) \in E_{s}
\end{aligned}
\end{gathered}
$$

Besides, the variables must hold the following bounds:

$$
\begin{gathered}
0 \leq \check{\phi}\left(v_{n}^{k}, v_{s}^{i}\right) \leq 1, \quad \forall i \in V_{s}, \forall n \in\left\{n \mid G_{n} \in \Phi\right\}, \forall k \in V_{n} \\
\alpha\left(\bar{v}_{s}^{i}\right), \alpha\left(\tilde{v}_{s}^{i}\right) \in\{0,1\}, \quad \forall i \in V_{s} \\
\alpha\left(l_{s}^{i, j}\right) \in\{0,1\}, \quad \forall(i, j) \in E_{s} \\
\alpha\left(\dot{v}_{n}^{k}, \bar{v}_{s}^{i}\right) \in\{0,1\}, \quad \forall i \in \bar{V}_{s}, \forall n \in\left\{n \mid G_{n} \in \Phi\right\}, \forall k \in \dot{V}_{n} \\
\alpha\left(\ddot{v}_{n}^{k}, \bar{v}_{s}^{i}\right) \in\{0,1\}, \quad \forall i \in \bar{V}_{s}, \forall n \in\left\{n \mid G_{n} \in \Phi\right\}, \forall k \in \ddot{V}_{n} \\
\alpha\left(\tilde{v}_{n}^{k}, \tilde{v}_{s}^{i}\right) \in\{0,1\}, \quad \forall i \in \tilde{V}_{s}, \forall n \in\left\{n \mid G_{n} \in \Phi\right\}, \forall k \in \tilde{V}_{n} \\
\alpha\left(l_{n}^{x, y}(m)\right), z^{i, j}\left(l_{n}^{x, y}(m)\right) \in\{0,1\}, \quad \forall x \in V_{s}, \forall y \in V_{s}, \\
\forall n \in\left\{n \mid G_{n} \in \Phi\right\}, m=1, \ldots, L_{n}
\end{gathered}
$$

The formulated MIDCP is a type of virtual network embedding problems. A virtual network embedding problem is $\mathcal{N} \mathcal{P}$-hard [15]. In consequence, the defined 
MIDCP is $\mathcal{N} \mathcal{P}$-hard, and it is not scalable to large network sizes.

\subsection{The Heuristic Algorithm}

Today's data centers include hundreds to thousands of physical servers. Therefore, we need to develop a heuristic algorithm for GreenMap that is scalable to large network sizes. The algorithm also has to achieve closely to the optimum points set by the formulated MIDCP. In this section, we propose such a heuristic for GreenMap.

The heuristic embeds MapReduce-based VNs onto the data center network, one by one, as the $\mathrm{VN}$ requests are received during the time. It maps a VN onto the data center network with the minimum additional energy consumption, and the hope of minimizing the VDC's total consumed energy at the end.

Four algorithms form our proposed heuristic for GreenMap. Each algorithm maps a part of the $n$th MapReduce-based virtual network onto the data center network. Algorithm 3 maps shuffler virtual nodes onto switch/router substrate nodes. Algorithm 4, and Algorithm 5 map reducer virtual nodes, and mapper virtual nodes onto server substrate nodes, respectively. Besides, Algorithm 6 maps virtual links onto substrate paths.

We first run Algorithm 3 to prepare the transit network for transferring generated intermediate data of computation-based virtual nodes. Afterwards, we run Algorithm 4 and map reducer virtual nodes onto server substrate nodes. Similar to the formulated MIDCP, the heuristic controls the incast queuing delay in every virtual link that terminates in a splitted and mapped reducer virtual node. Therefore, as it is discussed, we are limited regarding splitting and allocating reducer virtual nodes. However, we do not have such a limitation in embedding mapper virtual nodes. In consequence, in order to have more available resources in mapping reducer virtual nodes, we map reducer virtual nodes before embedding mapper virtual nodes. Afterwards, we run Algorithm 5 to allocate mapper virtual nodes in server substrate nodes. Algorithm 6 is called during the running process of the first three algorithms to embed the corresponding virtual links onto substrate paths.

\section{Mapping Shuffler Virtual Nodes}

A shuffler virtual node is allocated in a single switch/router substrate node. Multiple shuffler virtual nodes might be collocated in a single switch/router. In order to 
minimize the VDC's energy consumption, our priority is to map a shuffler virtual node onto an already active switch/router with the minimum power consumption for the requested switching capacity.

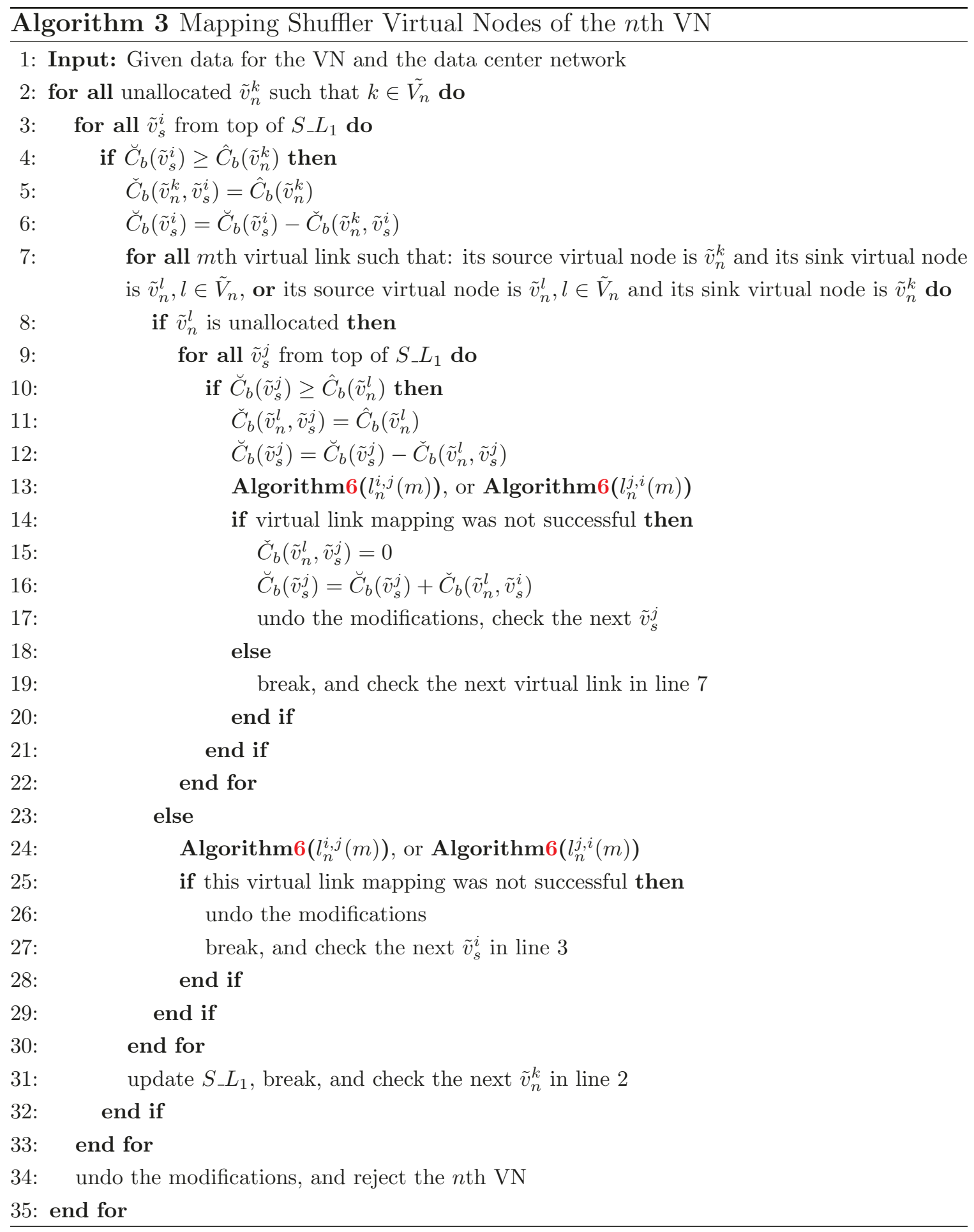


In this regard, we sort switch/router substrate nodes in a list called $S_{-} L_{1}$. Active switches/routers in $S_{-} L_{1}$ have a higher priority than inactive switches/routers. This is because activating an inactive switch/router adds a large amount of base power consumption. Active switches/routers are sorted in ascending order based on their value of $\frac{\tilde{p}^{m}\left(\tilde{v}_{s}^{i}\right)-\tilde{p}^{b}\left(\tilde{v}_{s}^{i}\right)}{C_{b}\left(\tilde{v}_{s}^{i}\right)}$. As a result, the same amount of switching demand adds a less amount of power consumption in higher priority switches/routers, according to Equation 5.2. If the value of this fraction is equal for some switches/routers, then we sort them in descending order based on their available switching capacity. A switch/router with a larger amount of available switching capacity may allow us to collocate a larger number of shuffler virtual nodes in it, and therefore allow us to save more energy. On the other hand, we sort inactive switches/routers in ascending order based on their base power consumption. Thus, if it is necessary to activate an inactive switch/router, then the lowest possible amount of base power consumption will be required. We sort inactive switches/routers with the equal amount of base power consumption in ascending order based on $\frac{\tilde{p}^{m}\left(\tilde{v}_{s}^{i}\right)-\tilde{p}^{b}\left(\tilde{v}_{s}^{i}\right)}{C_{b}\left(\tilde{v}_{s}^{i}\right)}$, because of the same discussed reason.

Algorithm 3 searches for a switch/router candidate $\tilde{v}_{s}^{i}$ from the top of $S_{-} L_{1}$ to map an unallocated shuffler virtual node $\tilde{v}_{n}^{k}$ onto it. A switch/router $\tilde{v}_{s}^{i}$ is a candidate if its available switching capacity $\breve{C}_{b}\left(\tilde{v}_{s}^{i}\right)$ is equal or greater than the switching capacity demand $\hat{C}_{b}\left(\tilde{v}_{n}^{k}\right)$ of the shuffler virtual node $\tilde{v}_{n}^{k}$. For such a candidate, the algorithm similarly maps every other unallocated shuffler virtual node that is connected to $\tilde{v}_{n}^{k}$ with a virtual link, onto a switch/router. In the next step, it calls Algorithm 6 to embed the candidate's adjacent virtual links, between the allocated shuffler virtual nodes, onto substrate paths. We describe Algorithm 6's process later in this section. If the algorithm successfully maps the adjacent shuffler virtual nodes and virtual links of the candidate, then the allocated switching capacity $\check{C}_{b}\left(\tilde{v}_{n}^{k}, \tilde{v}_{s}^{i}\right)$ to $\tilde{v}_{n}^{k}$ in $\tilde{v}_{s}^{i}$ is $\hat{C}_{b}\left(\tilde{v}_{n}^{k}\right)$. However, if it could not successfully map an unallocated shuffler virtual node onto a switch/router, then it rejects the VN.

\section{Mapping Reducer Virtual Nodes}

In the next step, we run Algorithm 4 to map reducer virtual nodes onto server substrate nodes. The algorithm splits the processing demand $\hat{C}_{c}\left(\ddot{v}_{n}^{k}\right)$ of a reducer virtual node $\ddot{v}_{n}^{k}$ and map the splitted demands onto servers, while it minimizes the VDC's energy consumption and controls the incast queuing delay. The way it splits the 
processing demands and map them onto servers has critical impacts on the energy consumption and the incast queuing delay.

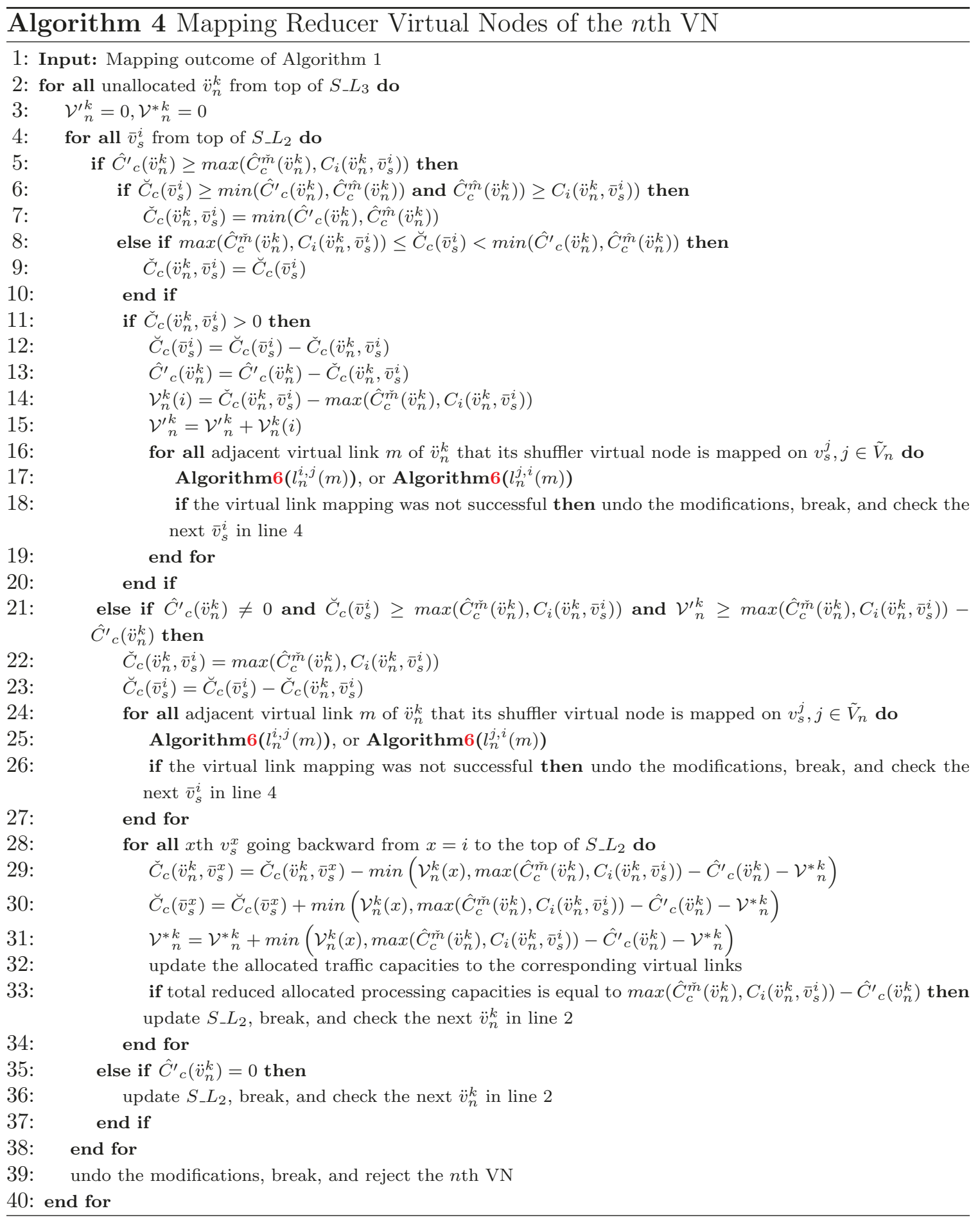


In order to reduce the VDC's energy consumption when we map reducer virtual nodes, we intend to use the minimum possible number of physical servers with the lowest power consumption for demanded processing capacities. In this regard, we sort server substrate nodes in a list called $S_{-} L_{2}$. In $S_{-} L_{2}$, active servers have a higher priority than inactive servers. Active servers are sorted in ascending order based on $\frac{\tilde{p}^{m}\left(\bar{v}_{s}^{i}\right)-\tilde{p}^{b}\left(\tilde{v}_{s}^{i}\right)}{C_{c}\left(\bar{v}_{s}^{i}\right)}$. This ensures the same amount of processing demand adds less power consumption in servers with a higher priority, according to Equation 5.1. Servers with the equal value of $\frac{\tilde{p}^{m}\left(\bar{v}_{s}^{i}\right)-\tilde{p}^{b}\left(\tilde{v}_{s}^{i}\right)}{C_{c}\left(\bar{v}_{s}^{i}\right)}$ are sorted based on their available processing capacity in descending order. A larger amount of available processing capacity in servers may allow us to split processing demands in larger blocks. Therefore, we require a smaller number of servers to allocate them in, and the algorithm is more flexible regarding the incast constraint. Besides, we sort inactive servers in ascending order based on their base power consumption. Thus, if we need to activate an inactive server, then the lowest possible amount of base power consumption is needed. Servers with the equal value of base power consumption are sorted in ascending order based on $\frac{\tilde{p}^{m}\left(\bar{v}_{s}^{i}\right)-\tilde{p}^{b}\left(\tilde{v}_{s}^{i}\right)}{C_{c}\left(\bar{v}_{s}^{i}\right)}$ for the same discussed reason. Note that we repeatedly update $S_{-} L_{2}$ during Algorithm 4's process, upon a reducer virtual node is mapped.

Moreover, we sort reducer virtual nodes in a list called $S_{-} L_{3}$, in descending order based on $\frac{\hat{C}_{c}\left(\ddot{v}_{n}^{k}\right)}{\hat{C}_{c}^{\check{m}}\left(\ddot{v}_{n}^{k}\right)}$. $\frac{\hat{C}_{c}\left(\ddot{v}_{n}^{k}\right)}{\hat{C}_{c}^{\check{m}}\left(\ddot{v}_{n}^{k}\right)}$ is the maximum number of distinct servers that may be required for mapping a reducer virtual node $\ddot{v}_{n}^{k}$. Algorithm 4 first maps reducer virtual nodes with a higher value of $\frac{\hat{C}_{c}\left(\ddot{v}_{n}^{k}\right)}{\hat{C}_{c}^{\check{m}}\left(\ddot{v}_{n}^{k}\right)}$ to increase the admittance ratio of the network.

In order to use the minimum number of servers with the lowest power consumption, we need to split the processing demand of a reducer virtual node $\ddot{v}_{n}^{k}$ into the largest possible processing blocks, and allocate them in the appropriate servers. The algorithm checks the ordered server substrate nodes in $S_{-} L_{2}$ and attempts to allocate the maximum possible processing capacity to an ordered unallocated reducer virtual node $\ddot{v}_{n}^{k}$ in $S_{-} L_{3}$ in a server. However, the allocated processing capacity $\check{C}_{c}\left(\ddot{v}_{n}^{k}, \bar{v}_{s}^{i}\right)$ to a reducer virtual node $\ddot{v}_{n}^{k}$ in a server substrate node $\bar{v}_{s}^{i}$, must satisfy two conditions. First, $\check{C}_{c}\left(\ddot{v}_{n}^{k}, \bar{v}_{s}^{i}\right)$ must be equal or greater than the given $\hat{C}_{c}^{\check{m}}\left(\ddot{v}_{n}^{k}\right)$ and equal or less than the known $\hat{C}_{c}^{\hat{m}}\left(\ddot{v}_{n}^{k}\right)$. Second, $\check{C}_{c}\left(\ddot{v}_{n}^{k}, \bar{v}_{s}^{i}\right)$ must be greater than the minimum incast processing capacity $C_{i}\left(\ddot{v}_{n}^{k}, \bar{v}_{s}^{i}\right)$, which guarantees an in range (less than $\hat{D}$ ) end-to-end queuing delay for the incast traffic pattern in the substrate path allocated to a virtual link that terminates at $\ddot{v}_{n}^{k}$ in $\bar{v}_{s}^{i}$. 
We assumed that a reducer virtual node is connected to the rest of the network via a shuffler virtual node in a VN topology. Besides, shuffler virtual nodes are already mapped in the previous step. Therefore, it is possible to calculate the minimum incast processing capacity $C_{i}\left(\ddot{v}_{n}^{k}, \bar{v}_{s}^{i}\right)$ when we check the suitability of a server $\bar{v}_{s}^{i}$ for a $\ddot{v}_{n}^{k}$, analytically. In order to calculate $C_{i}\left(\ddot{v}_{n}^{k}, \bar{v}_{s}^{i}\right)$, we use Algorithm 6 to find substrate paths that will be allocated to each sub virtual link which connects an allocated shuffler virtual node $\tilde{v}_{n}^{l}$ to $\ddot{v}_{n}^{k}$. Note that a reducer virtual node may be connected to one or multiple shuffler virtual nodes in a VN's topology. The mean end-toend incast queuing delay in the longest found substrate path determines $C_{i}\left(\ddot{v}_{n}^{k}, \bar{v}_{s}^{i}\right)$. $\underline{L}\left(\ddot{v}_{n}^{k}, \bar{v}_{s}^{i}\right)$ denotes the number of physical links in the found longest substrate path. We do not allocate any traffic capacity at this stage. According to $M / M / 1$ queue and Jackson Networks theorem, and because we do not split generated traffic by an allocated virtual node, the minimum required bandwidth capacity that needs to be allocated to the virtual link $l_{n}^{l, k}$ with the longest substrate path, is:

$$
\frac{\underline{L}\left(\ddot{v}_{n}^{k}, \bar{v}_{s}^{i}\right)}{\hat{D}}+\lambda_{n}^{\mathcal{M}}
$$

As it is discussed before, we know this value is equal to $\check{\phi}\left(\ddot{v}_{n}^{k}, \bar{v}_{s}^{i}\right) \hat{C}_{b}\left(l l_{n}^{l, k}\right)$. Hence,

$$
\begin{aligned}
& \check{\phi}\left(\ddot{v}_{n}^{k}, \bar{v}_{s}^{i}\right)=\frac{\frac{\underline{\underline{L}}\left(\ddot{v}_{n}^{k}, \bar{v}_{s}^{i}\right)}{\hat{D}}+\lambda_{n}^{\mathcal{M}}}{\hat{C}_{b}\left(l_{n}^{l, k}\right)}, \\
& \frac{C_{i}\left(\ddot{v}_{n}^{k}, \bar{v}_{s}^{i}\right)}{\hat{C}_{c}\left(\ddot{v}_{n}^{k}\right)}=\frac{\frac{\underline{\underline{L}}\left(\ddot{v}_{n}^{k}, \bar{v}_{s}^{i}\right)}{\hat{D}}+\lambda_{n}^{\mathcal{M}}}{\hat{C}_{b}\left(l_{n}^{l, k}\right)}, \\
& C_{i}\left(\ddot{v}_{n}^{k}, \bar{v}_{s}^{i}\right)=\frac{\hat{C}_{c}\left(\ddot{v}_{n}^{k}\right)}{\hat{C}_{b}\left(l_{n}^{l, k}\right)}\left(\frac{\underline{L}\left(\ddot{v}_{n}^{k}, \bar{v}_{s}^{i}\right)}{\hat{D}}+\lambda_{n}^{\mathcal{M}}\right)
\end{aligned}
$$

Furthermore, $\hat{C}^{\prime}{ }_{c}\left(\ddot{v}_{n}^{k}\right)$ is the remained processing capacity that needs to be allocated to $\ddot{v}_{n}^{k}$. At the first, $\hat{C}^{\prime}{ }_{c}\left(\ddot{v}_{n}^{k}\right)$ equals to $\hat{C}_{c}\left(\ddot{v}_{n}^{k}\right)$. $\hat{C}^{\prime}{ }_{c}\left(\ddot{v}_{n}^{k}\right)$ might be changed as the algorithm processes the ordered servers for the possible mapping. In this regard, we might face three conditions based on $\hat{C}^{\prime}{ }_{c}\left(\ddot{v}_{n}^{k}\right)$, at each time we check a $\bar{v}_{s}^{i}$ to map $\ddot{v}_{n}^{k}$ onto it.

First, $\hat{C}_{c}^{\prime}\left(\ddot{v}_{n}^{k}\right)$ is larger than $\max \left(\hat{C}_{c}^{\check{m}}\left(\ddot{v}_{n}^{k}\right), C_{i}\left(\ddot{v}_{n}^{k}, \bar{v}_{s}^{i}\right)\right)$. In this case, allocating the remained processing demand to $\ddot{v}_{n}^{k}$ in $\bar{v}_{s}^{i}$ does not violate either the minimum requested processing capacity per physical machine $\hat{C}_{c}^{\check{m}}\left(\ddot{v}_{n}^{k}\right)$, or the maximum tolerable queuing 
delay $\hat{D}$ in the adjacent virtual links. Therefore, as Figure 5.2 shows, we allocate the maximum processing capacity based on the available processing capacity $\breve{C}_{c}\left(\bar{v}_{s}^{i}\right)$ in the server $\bar{v}_{s}^{i}$. If $\breve{C}_{c}\left(\bar{v}_{s}^{i}\right)$ is equal or greater than $\min \left(\hat{C}_{c}^{\prime}\left(\ddot{v}_{n}^{k}\right), \hat{C}_{c}^{\hat{m}}\left(\ddot{v}_{n}^{k}\right)\right)$, then the maximum processing capacity we could allocate to $\ddot{v}_{n}^{k}$ in $\bar{v}_{s}^{i}$ is the smaller value between $\hat{C}_{c}^{\prime}\left(\ddot{v}_{n}^{k}\right)$ and $\hat{C}_{c}^{\hat{m}}\left(\ddot{v}_{n}^{k}\right)$. This ensures we do not allocate a larger amount than the requested $\hat{C}_{c}^{\hat{m}}\left(\ddot{v}_{n}^{k}\right)$, or $\hat{C}_{c}{ }_{c}\left(\ddot{v}_{n}^{k}\right)$. Of course, $\hat{C}_{c}^{\hat{m}}\left(\ddot{v}_{n}^{k}\right)$ must be equal or greater than $C_{i}\left(\ddot{v}_{n}^{k}, \bar{v}_{s}^{i}\right)$. But, if $\breve{C}_{c}\left(\bar{v}_{s}^{i}\right)$ is less than $\min \left(\hat{C}_{c}^{\prime}\left(\ddot{v}_{n}^{k}\right), \hat{C}_{c}^{\hat{m}^{2}}\left(\ddot{v}_{n}^{k}\right)\right)$ and equal or greater than $\max \left(\hat{C}_{c}^{\check{m}}\left(\ddot{v}_{n}^{k}\right), C_{i}\left(\ddot{v}_{n}^{k}, \bar{v}_{s}^{i}\right)\right)$, then the maximum processing capacity we could allocate to $\ddot{v}_{n}^{k}$ in $\bar{v}_{s}^{i}$ is $\breve{C}_{c}\left(\bar{v}_{s}^{i}\right)$. So, it does not violate the minimum/maximum requested processing capacity per physical machine, or the maximum tolerable queuing delay $\hat{D}$ in the adjacent virtual links. Otherwise, no processing capacity is allocated to $\ddot{v}_{n}^{k}$ in $\bar{v}_{s}^{i}$. If the algorithm allocates a processing capacity in a server, then it calls Algorithm 6 to map the corresponding sub virtual links.

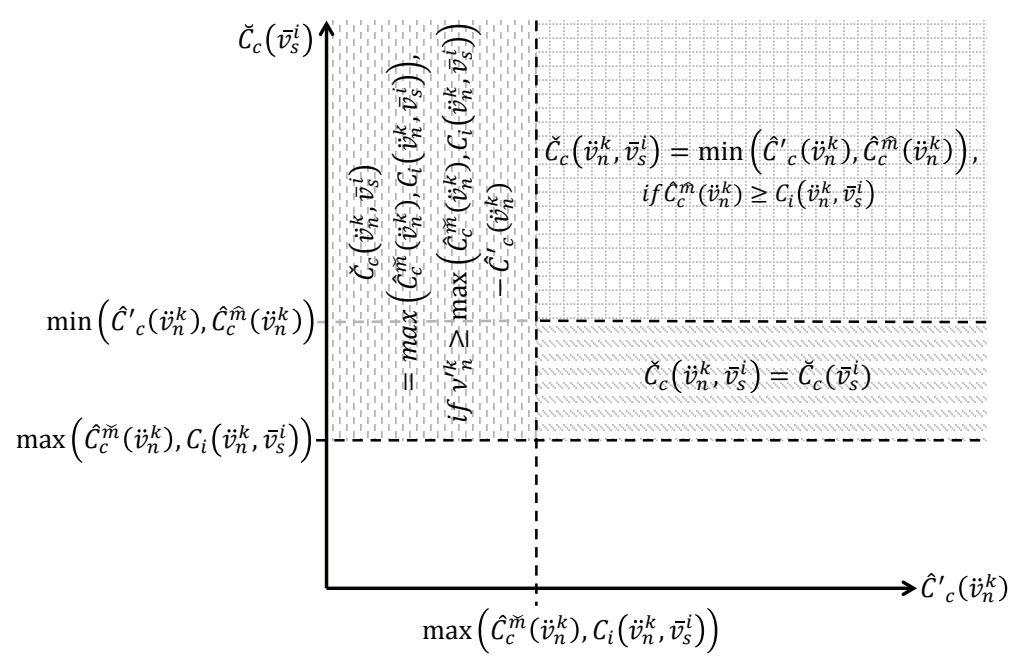

Figure 5.2: The heuristic's decision making process

Second, $\hat{C}_{c}^{\prime}\left(\ddot{v}_{n}^{k}\right)$ is smaller than $\max \left(\hat{C}_{c}^{\check{m}}\left(\ddot{v}_{n}^{k}\right), C_{i}\left(\ddot{v}_{n}^{k}, \bar{v}_{s}^{i}\right)\right)$. This means if we allocate $\hat{C}_{c}^{\prime}\left(\ddot{v}_{n}^{k}\right)$ to $\ddot{v}_{n}^{k}$ in $\bar{v}_{s}^{i}$, then either the minimum requested processing capacity per physical machine $\hat{C}_{c}^{\check{m}}\left(\ddot{v}_{n}^{k}\right)$, or the maximum tolerable queuing delay $\hat{D}$ in its adjacent virtual links, will be violated. Instead, the algorithm allocates $\max \left(\hat{C}_{c}^{\check{m}}\left(\ddot{v}_{n}^{k}\right), C_{i}\left(\ddot{v}_{n}^{k}, \bar{v}_{s}^{i}\right)\right)$ to $\ddot{v}_{n}^{k}$ in $\bar{v}_{s}^{i}$ and maps its corresponding sub virtual links, if $\bar{v}_{s}^{i}$ has enough available processing capacity. $\max \left(\hat{C}_{c}^{\check{m}}\left(\ddot{v}_{n}^{k}\right), C_{i}\left(\ddot{v}_{n}^{k}, \bar{v}_{s}^{i}\right)\right)-\hat{C}_{c}^{\prime}{ }_{c}\left(\ddot{v}_{n}^{k}\right)$ is the extra processing capacity 
allocated to $\ddot{v}_{n}^{k}$ in $\bar{v}_{s}^{i}$. Therefore, the algorithm recursively updates the previously allocated processing capacities to $\ddot{v}_{n}^{k}$ in servers to decrease the extra allocated capacity, if the constraints (the requested range of processing capacity per physical machine, and the maximum tolerable delay in the adjacent virtual links) will not be violated.

In this regard, when a processing capacity is allocated to a virtual node in a server, we record the amount of processing capacity that if we subtract it from the allocated processing capacity, then none of the constraints will be violated. This amount for a $\ddot{v}_{n}^{k}$ in a $\bar{v}_{s}^{i}$ is equal to $\check{C}_{c}\left(\ddot{v}_{n}^{k}, \bar{v}_{s}^{i}\right)-\max \left(\hat{C}_{c}^{\check{m}}\left(\ddot{v}_{n}^{k}\right), C_{i}\left(\ddot{v}_{n}^{k}, \bar{v}_{s}^{i}\right)\right)$, and it is represented by $\mathcal{V}_{n}^{k}(i) . \mathcal{V}_{n}^{\prime k}$ is total $\mathcal{V}_{n}^{k}(i)$ for $\ddot{v}_{n}^{k}$.

We decrease the previously allocated processing capacities to $\ddot{v}_{n}^{k}$ in servers (e.g. $\left.\bar{v}_{s}^{x}\right)$ by $\min \left(\mathcal{V}_{n}^{k}(x), \max \left(\hat{C}_{c}^{\check{m}^{m}}\left(\ddot{v}_{n}^{k}\right), C_{i}\left(\ddot{v}_{n}^{k}, \bar{v}_{s}^{i}\right)\right)-\hat{C}^{\prime}{ }_{c}\left(\ddot{v}_{n}^{k}\right)-\mathcal{V}_{n}^{* k}\right)$, until the extra allocated capacity is removed. This is the case if $\mathcal{V}_{n}^{\prime k}$ is equal or greater than the extra allocated capacity. Then, the algorithm updates the allocated traffic capacities to the corresponding sub virtual links. Note that $\mathcal{V}_{n}^{* k}$ is the total removed processing capacity for $\ddot{v}_{n}^{k}$.

Third, $\hat{C}^{\prime}{ }_{c}\left(\ddot{v}_{n}^{k}\right)$ is 0 . This means $\ddot{v}_{n}^{k}$ is mapped successfully.

Algorithm 4 rejects the VN, if it could not successfully map a reducer virtual node and its adjacent virtual links onto servers and substrate paths, respectively.

\section{Mapping Mapper Virtual Nodes}

We run Algorithm 5 as the last step, to embed mapper virtual nodes onto physical servers. Algorithm 5 works similarly to Algorithm 4. However, there are some minor differences.

Different from the mapping process of a reducer virtual node, we do not concern about the incast queuing delay when we map a mapper virtual node onto servers. Therefore, in contrary to Algorithm 4, Algorithm 5 does not check the minimum incast processing capacity during its allocation process. This simplifies the mapping procedure. As a result, the recursive process in Algorithm 5, which modifies the allocated capacities due to the extra allocated processing capacity, is slightly different from the recursive process in Algorithm 4. The extra allocated processing capacity to a $\dot{v}_{n}^{k}$ in a $\bar{v}_{s}^{i}$ in Algorithm 5 is $\hat{C}_{c}^{\check{m}}\left(\dot{v}_{n}^{k}\right)-\hat{C}^{\prime}{ }_{c}\left(\dot{v}_{n}^{k}\right)$, where $\hat{C}^{\prime}{ }_{c}\left(\dot{v}_{n}^{k}\right)$ is the remained processing capacity that needs to be allocated to $\dot{v}_{n}^{k}$. This is because the minimum processing capacity that could be allocated to a $\dot{v}_{n}^{k}$ in a $\bar{v}_{s}^{i}$ is only limited by $\hat{C}_{c}^{\check{m}}\left(\dot{v}_{n}^{k}\right)$. Besides, Algorithm 5 searches in the previously allocated processing capacities to find 
the one which if it subtracts the extra allocated processing capacity from the already allocated processing capacity, then the result does not violate the minimum requested processing capacity per physical machine. Note that $\check{C}_{c}\left(\dot{v}_{n}^{k}, \bar{v}_{s}^{i}\right)$ is the allocated processing capacity to $\dot{v}_{n}^{k}$ in $\bar{v}_{s}^{i}$.






\section{Mapping Virtual Links}

We have seen Algorithms 3, 4, 5, might call Algorithm 6 to allocate a virtual link or a sub virtual link $l_{n}^{i, j}(m)$ in a substrate path. In this regard, Algorithm 6 finds $K$ loopless shortest path from $v_{s}^{i}$ to $v_{s}^{j}$ in the data center network. If source and sink substrate nodes of a substrate link are active, then the cost of the substrate link is 1. Otherwise, the substrate link has a large cost, e.g. 100. This helps to find the shortest paths with activating the minimum number of inactive substrate links and nodes. Our preferred routing algorithm to find $K$ loopless shortest paths is the very well known Yen's algorithm [91]. It is also possible to consider more recent methods of finding $K$ loopless shortest paths as proposed in $[110,111]$. Note that the value of $K$ is adjustable. It will be more probable to find a capable substrate path, by incrementing the value of $K$. However, incrementing the value of $K$ increases the time complexity of the algorithm, as will be discussed later in this chpater. The right value for $K$ could be chosen according to the size of the data center network.

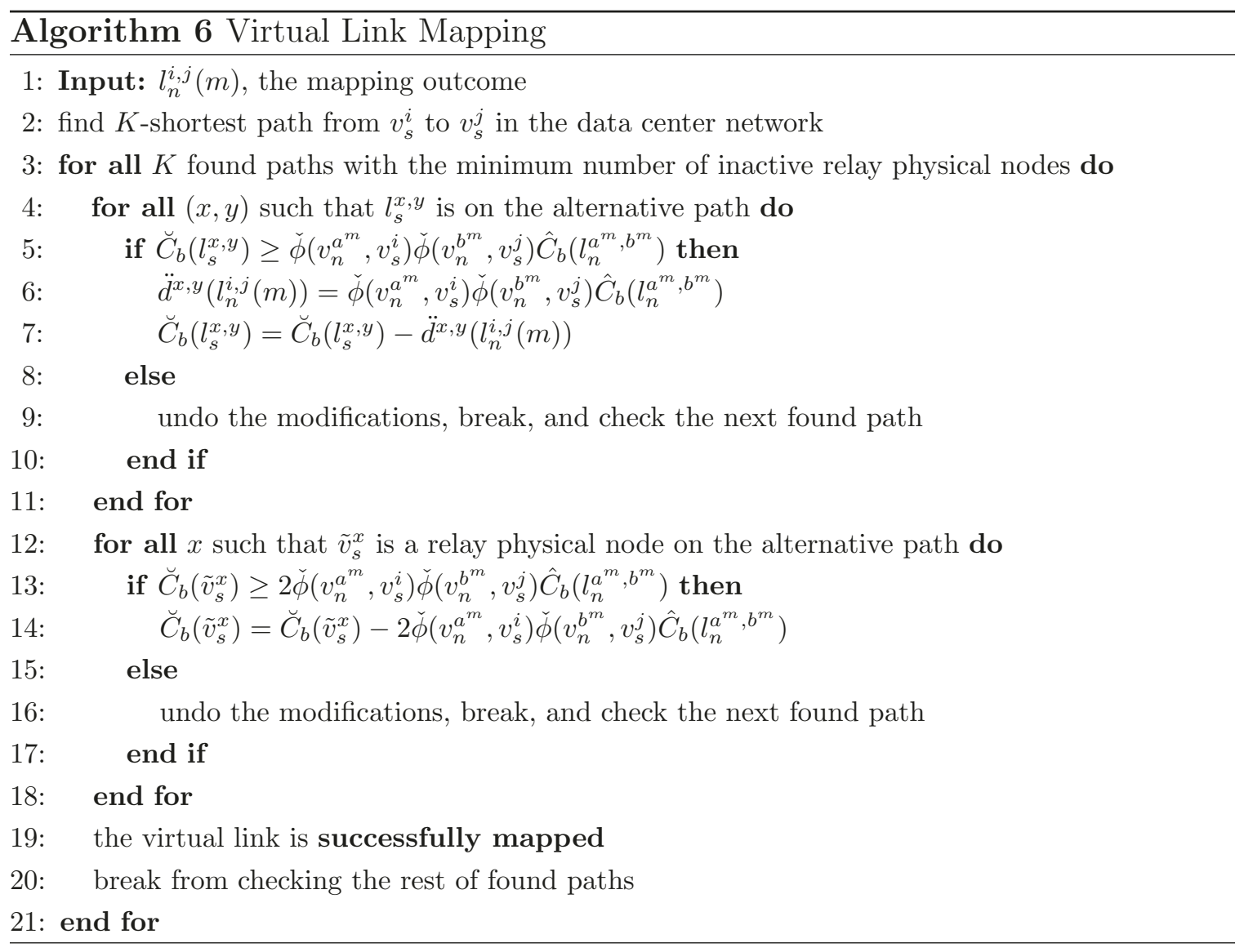


Algorithm 6 may check the capability of every found substrate path, regarding the required traffic capacity. We know the traffic capacity that needs to be allocated to $l_{n}^{i, j}(m)$ is equal to $\check{\phi}\left(v_{n}^{a^{m}}, v_{s}^{i}\right) \check{\phi}\left(v_{n}^{b^{m}}, v_{s}^{j}\right) \hat{C}_{b}\left(l_{n}^{a^{m}, b^{m}}\right)$. Therefore, a substrate path is a candidate if the available traffic capacity $\breve{C}_{b}\left(l_{s}^{x, y}\right)$ in every physical link $l_{s}^{x, y}$ along the path is equal or greater than $\check{\phi}\left(v_{n}^{a^{m}}, v_{s}^{i}\right) \check{\phi}\left(v_{n}^{b^{m}}, v_{s}^{j}\right) \hat{C}_{b}\left(l_{n}^{a^{m}, b^{m}}\right)$. Besides, the available switching capacity $\breve{C}_{b}\left(\tilde{v}_{s}^{x}\right)$ in every relay substrate node $\tilde{v}_{s}^{x}$ along the candidate path also must be equal or grater than $2 \check{\phi}\left(v_{n}^{a^{m}}, v_{s}^{i}\right) \check{\phi}\left(v_{n}^{b^{m}}, v_{s}^{j}\right) \hat{C}_{b}\left(l_{n}^{a^{m}, b^{m}}\right)$. This considers the incoming and outgoing traffic in a relay substrate node. Note that a server can not be a relay substrate node in our defined data center network topology. The virtual link or the sub virtual link is successfully mapped onto the data center network, if the algorithm could find such a substrate path for it.

It is required to find the time complexity of the proposed heuristic to see if it is scalable to large network sizes. In this regard, we need to find the complexity of each of the described algorithms for mapping the $n$th MapReduced-based VN onto the data center network.

We first find the complexity of Algorithm 6, because it is called during the other algorithms. Algorithm 6 calls Yen's algorithm in line 2. The complexity of Yen's algorithm is $\mathcal{O}\left(K\left|V_{s}\right|\left(\left|E_{s}\right|+\left|V_{s}\right| \log \left|V_{s}\right|\right)\right.$. Besides, the loop that starts in line 3 and ends in line 21 is run for $K$ times. This loop has two sub-loops. The first sub-loop that starts in line 4 and ends in line 11 is run for every substrate link in the worstcase. The algorithm inside this sub-loop might undo some modifications, that in the worst-case, it checks every substrate link again. Hence, the complexity of this sub-loop is $\mathcal{O}\left(\left|E_{s}\right|^{2}\right)$. The second sub-loop that starts in line 12 and ends in line 18 is run for every switch/router substrate node in the worst-case. Inside this sub-loop the algorithm may also undo some modifications. So, it may need to update every substrate link and switch/router again. Thus, the complexity of the second sub-loop is $\mathcal{O}\left(\left|\tilde{V}_{s}\right|\left(\left|E_{s}\right|+\left|\tilde{V}_{s}\right|\right)\right)$. All in all, considering only the dominating factors, the time complexity of Algorithm 6 is $\mathcal{O}\left(K\left(\left|V_{s}\right|\left(\left|E_{s}\right|+\left|V_{s}\right| \log \left|V_{s}\right|\right)+\left|E_{s}\right|^{2}\right)\right)$.

The complexity of Algorithm 3 is determined by the main loop that starts in line 2 and ends in line 35. This loop is run for every shuffler virtual node in the VN. So, it is run for $\left|\tilde{V}_{n}\right|$ times. A sub-loop of the main loop starts in line 3 and ends in line 33. This sub-loop is run for $\left|\tilde{V}_{s}\right|$ in the worst-case. Besides, we have a sub-loop in Algorithm 3 which starts in line 7 and ends in line 30. This sub-loop maps every adjacent virtual link of a shuffler virtual node. In the worst-case, it is run for $\left|E_{n}\right|$ 
times. The other sub-loop that starts in line 9 and ends in line 22 may be run for $\left|\tilde{V}_{s}\right|$ times. The algorithm may call Algorithm 6 to map the corresponding virtual links. We know the complexity of Algorithm 6 is $\mathcal{O}\left(K\left(\left|V_{s}\right|\left(\left|E_{s}\right|+\left|V_{s}\right| \log \left|V_{s}\right|\right)+\left|E_{s}\right|^{2}\right)\right)$. Besides, it might undo some modifications related to the shuffler virtual node and its mapped virtual links. So, the complexity of this function is $\mathcal{O}\left(\left|\tilde{V}_{s}\right|+\left|E_{s}\right|\left|E_{n}\right|\right)$. Consequently, by taking into account the dominating factors, the time complexity of Algorithm 3 is $\mathcal{O}\left(\left|\tilde{V}_{n}\right|\left|\tilde{V}_{s}\right|^{2}\left|E_{n}\right|\left(K\left|V_{s}\right|\left|E_{s}\right|+K\left|V_{s}\right|^{2} \log \left|V_{s}\right|+K\left|E_{s}\right|^{2}+\left|E_{s}\right|\left|E_{n}\right|\right)\right)$.

The main loop that starts in line 2 and ends in line 30 of Algorithm 4 specifies its time complexity. This loops is run for every reducer virtual node in the VN, which is $\left|\ddot{V}_{n}\right|$ times. A sub-loop that starts in line 4 and ends in line 37 may check the capability of every server substrate node for the reducer virtual node. So, it is run for $\left|\bar{V}_{s}\right|$ times. We have another sub-loop that starts in line 16 and ends in line 19. A similar sub-loop also starts in line 24 and ends in line 27. Both of them are run for $\left|E_{n}\right|$ times in the worst-case. Algorithm 6 is called inside these sub-loops. Besides, the algorithm may undo some modifications inside these sub-loops. Here, the undoing function may check every server substrate node for the allocated processing capacities to the reducer virtual node, and each substate link for the allocated traffic capacities to the virtual links. Therefore, its complexity is $\mathcal{O}\left(\left|\bar{V}_{s}\right|+\left|E_{s}\right|\left|E_{n}\right|\right)$. The other subloop that starts in line 28 and ends in line 33 recursively updates the previously allocated capacities. This sub-loop may be run for $\left|\bar{V}_{s}\right|$ times. The algorithm also updates the previously allocated traffic capacities to the corresponding virtual links inside this sub-loop. The complexity of this function is $\mathcal{O}\left(\left|E_{s}\right|\left|E_{n}\right|\right)$. Hence, taking into consideration the dominating factors, the time complexity of Algorithm 4 is $\mathcal{O}\left(\left|\ddot{V}_{n}\right|\left|\bar{V}_{s}\right|\left|E_{n}\right|\left(K\left|\bar{V}_{s}\right|\left|E_{s}\right|+K\left|\bar{V}_{s}\right|^{2} \log \left|\bar{V}_{s}\right|+K\left|E_{s}\right|^{2}+\left|E_{s}\right|\left|E_{n}\right|\right)\right)$.

The time complexity of Algorithm 5 could be derived similarly to the time complexity of Algorithm 4. The only difference is that the main loop in Algorithm 5 is run for every mapper virtual node instead of every reducer virtual node in Algorithm 4. Therefore, it is run for $\left|\dot{V}_{n}\right|$ times. In consequence, the time complexity of Algorithm 5 is $\mathcal{O}\left(\left|\dot{V}_{n}\right|\left|\bar{V}_{s}\right|\left|E_{n}\right|\left(K\left|\bar{V}_{s}\right|\left|E_{s}\right|+K\left|\bar{V}_{s}\right|^{2} \log \left|\bar{V}_{s}\right|+K\left|E_{s}\right|^{2}+\left|E_{s}\right|\left|E_{n}\right|\right)\right)$.

We know Algorithms 3, 4, and 5 are run in series to form the heuristic for GreenMap. Thus, the time complexity of the heuristic for mapping $n$th $\mathrm{VN}$ is $\mathcal{O}\left(\left(\left|\tilde{V}_{n}\right|\left|\tilde{V}_{s}\right|^{2}\left|E_{n}\right|\left(K\left|V_{s}\right|\left|E_{s}\right|+K\left|V_{s}\right|^{2} \log \left|V_{s}\right|+K\left|E_{s}\right|^{2}+\left|E_{s}\right|\left|E_{n}\right|\right)\right)+\right.$ $\left(\left|\ddot{V}_{n}\right|\left|\bar{V}_{s}\right|\left|E_{n}\right|\left(K\left|\bar{V}_{s}\right|\left|E_{s}\right|+K\left|\bar{V}_{s}\right|^{2} \log \left|\bar{V}_{s}\right|+K\left|E_{s}\right|^{2}+\left|E_{s}\right|\left|E_{n}\right|\right)\right)+\left(\left|\dot{V}_{n}\right|\left|\bar{V}_{s}\right|\left|E_{n}\right|\left(K\left|\bar{V}_{s}\right|\left|E_{s}\right|+\right.\right.$ $\left.\left.\left.K\left|\bar{V}_{s}\right|^{2} \log \left|\bar{V}_{s}\right|+K\left|E_{s}\right|^{2}+\left|E_{s}\right|\left|E_{n}\right|\right)\right)\right)$. This proves the heuristic could be solved in a 
polynomial time.

\subsection{Evaluation}

GreenMap is supposed to map the heterogeneous MapReduce-based VNs onto the heterogeneous data center network, and minimize its total energy consumption. Besides, it needs to control the introduced incast queueing delay. We verify the performance of the formulated MIDCP and the proposed heuristic for GreenMap by generating and mapping random MapReduce-based VNs onto a data center network with a multi-level topology.

As it is discussed, the formulated MIDCP is $\mathcal{N} \mathcal{P}$-hard, so it is not scalable to large network sizes. Therefore, similar to the other related works in $[17,56,69,70]$, we assess the capability of the MIDCP on small random simulation setups. It is possible to solve mixed integer disciplined convex programs by combination of a continuous optimization algorithm and and an exhaustive search method [109]. We solved the formulated MIDCP by MOSEK solver [88]. Nonetheless, the theoretical complexity analysis reveals that the proposed heuristic algorithm is considerably simpler, and therefore it is scalable to large network sizes. Hence, the performance of the suggested heuristic is examined on large random simulation setups.

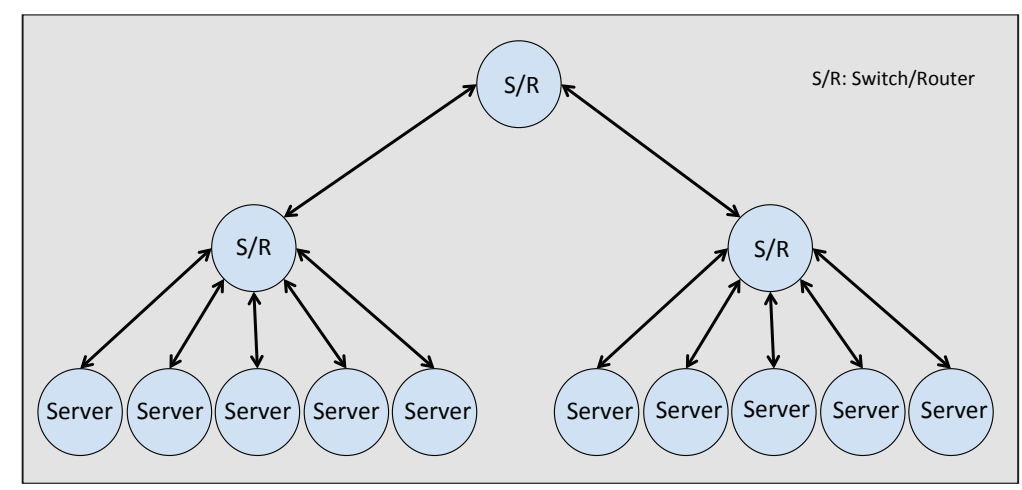

Figure 5.3: Topology of the data center network in a small simulation setup

A small random simulation setup includes a heterogeneous data center network with the symmetric tree topology. Figure 5.3 shows the topology of this network. It has 13 nodes including 10 servers and 3 switches/routers. We assume that servers are blades with CPU capacity of $2 \mathrm{GHz}$. The blade server is ideal for our study, because 
it is widely deployed in data centers and incorporates several power management techniques [59]. The base power consumption of a blade server is 213Watt, and its maximum power consumption is 319.5Watt [59]. It is assumed that a switch/router has $10 \mathrm{Gbps}$ switching capacity, and a physical link has $1 \mathrm{Gbps}$ bandwidth capacity. In this case, the base power consumption of a physical link is 1.7Watt, and its maximum power consumption is 2 Watt [56].

Recently, Waxman algorithm [84] is widely used by the researchers to generate random virtual network topologies $[17,69]$. Therefore, in this chapter, virtual networks' topologies are generated by Waxman algorithm. Waxman generates random network topologies based on two parameters. As the first parameter grows, the probability of having an edge between any pair of nodes in the topology is increased. We choose 0.4 for the first parameter. As the second parameter grows there is a larger ratio of long edges to short edges. We choose 0.2 for the second parameter.

In small random simulation setups, a virtual link's bandwidth demand is generated randomly between 100Mbps and 200Mbps, following the uniform distribution. The switching demand of a shuffler virtual node is assumed to be equal to the summation of all its incoming and outgoing virtual links' bandwidth demands. Moreover, $\lambda\left(\dot{v}_{n}^{k}\right)$ of a mapper virtual node $\dot{v}_{n}^{k}$ is generated with the uniform distribution between $1 \mathrm{Mbps}$ and $10 \mathrm{Mbps}$. We also assume that $\hat{D}$ is $25 \mathrm{msec}$. Note that no restrictions is considered for the minimum/maximum processing capacity per physical machine for a computationbased virtual node, unless otherwise stated.

A large random simulation setup includes a heterogeneous data center network with a fat-tree topology. Here, we consider a 6-ary fat-tree topology with three layers of edge, aggregation, and core. This topology is built by 6-port commodity switches/routers. It has 6 pods, each contains two layers (edge/aggregation) of 3 switches/routers. Every 6-port edge switch/router is connected to 3 servers, and 3 aggregation switches/routers. We also have 9 core switches/routers. Each 6port aggregation switch/router is connected to 3 edge switches/routers, and 3 core switches/routers. Every core switch/router is connected to each of 6 pods. The $i$ th port of a core switch/router is connected to the $i$ th pod. Thus, this topology has 54 servers, and 45 switches/routers. Similar to the small random simulation setup, servers are blades with CPU capacity of $2 \mathrm{GHz}$. It is assumed that a switch/router has $6 \times 10 \mathrm{Gbps}$ switching capacity, and a physical link has 10Gbps bandwidth capacity. In this case, the base and maximum power consumption of a physical link is assumed 
to be 17Watt and 20Watt, respectively [112].

In a large random simulation setup, a virtual link's bandwidth demand is generated randomly between $300 \mathrm{Mbps}$ and $500 \mathrm{Mbps}$, following the uniform distribution. Besides, $\hat{D}$ is assumed to be $50 \mathrm{msec}$. The rest of the configurations for large random simulation setups are the same as the small random simulation setups, unless otherwise instructed.

\subsubsection{The MIDCP}

First, we solved the MIDCP and the heuristic for GreenMap, and also the state-of-theart energy-efficient VN embedding algorithm in [69], for different numbers of virtual nodes per $\mathrm{VN}$, on a small random simulation setup. We measured the total power consumption by servers in all the cases. There is no existing approach that could split virtual nodes and embed heterogeneous MapReduce-based onto a heterogeneous data center network. So, we compared our solution with the well-cited energy-efficient VN embedding algorithm in [69], which does not allow node splitting. The number of virtual nodes per $\mathrm{VN}$ is ranged from 5 to 8, while each $\mathrm{VN}$ has 3 shuffler virtual nodes. It also has at least 1 mapper and 1 reducer virtual node, but the exact numbers are chosen randomly following the uniform distribution. We tested 10 randomly generated virtual networks for each number of virtual nodes per $\mathrm{VN}$, and plotted the average results including confidence intervals with the confidence level of 90\%, in Figure 5.4. A confidence interval is calculated by Equation 3.22.

In this simulation setup, the CPU demand of a computation-based virtual node is a random value between $500 \mathrm{MHz}$ and $1,500 \mathrm{MHz}$, following the uniform distribution. Note that this range is chosen, so the state-of-the-art algorithm is able to map a computation-based virtual node onto a single server substrate node.

The results in Figure 5.4 first confirm that the MIDCP and the heuristic for GreenMap effectively reduce the total power consumption by servers, in comparison to the state-of-the-art energy-efficient mapping algorithm. This is because the stateof-the-art algorithm does not allow either node splitting, or node collocation. Second, the results show that incrementing the number of virtual nodes per VN, increases the total consumed power by servers in the data center network. This is because we have a fixed number of shuffler virtual nodes and an increasing number of computation-based virtual nodes, while a computation-based virtual node needs some power to operate. Besides, the power consumption is increasing linearly in the case of state-of-the-art 
algorithm, since it maps each virtual node on a single substrate node. However, this is not the case for the MIDCP and the heuristic for GreenMap, as they splits the processing demands of computation-based virtual nodes and might map them onto multiple virtual nodes. They also may collocate multiple virtual nodes of a VN in a single substrate node.

Moreover, Figure 5.4 verifies that the heuristic can achieve reasonably close to the optimum results of the MIDCP. This is the case while the heuristic is considerably faster than the MIDCP. For example, in the same setup and for a single run, when we have 6 virtual nodes in a VN, the MIDCP's run time is 84,128 seconds. However, this amount for the heuristic is only 0.0414 second.

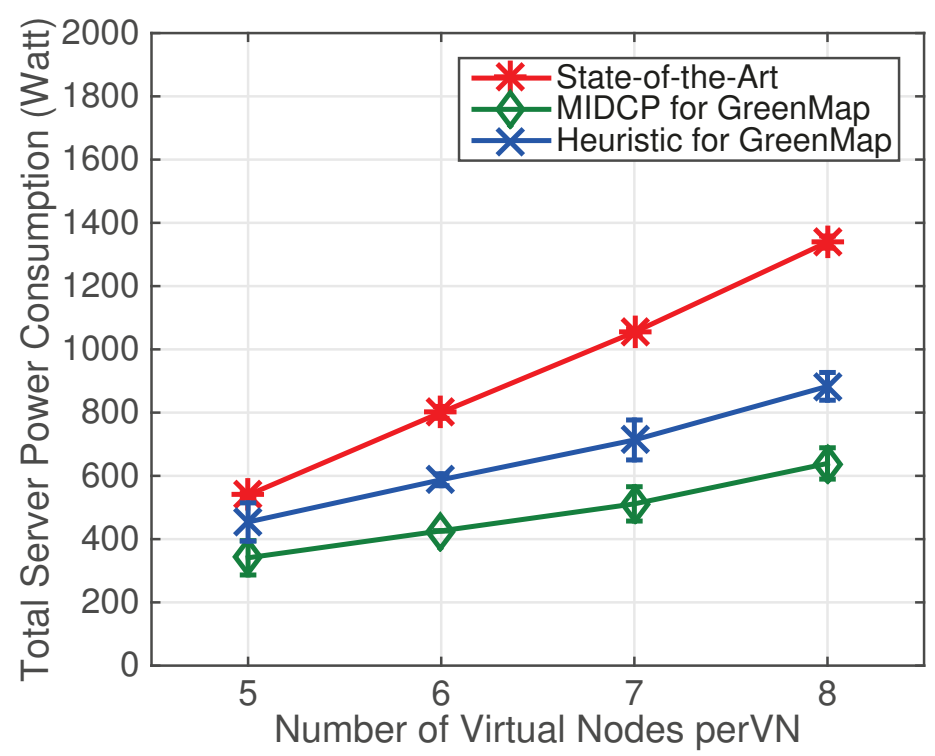

Figure 5.4: The total power consumption based on different numbers of virtual nodes per VN for the state-of-the-art algorithm, the MIDCP for GreenMap, and the heuristic for GreenMap

In addition, we solved the formulated MIDCP for GreenMap for different CPU demands of a computation-based virtual node, on a small random simulation setup. This is also solved for when the incast constraints in Equation 5.20 are relaxed. We measured the mean incast queuing delay in the allocated substrate paths to virtual links that terminate at a reducer virtual node, for both cases. The CPU demands are ranged from $2,000 \mathrm{MHz}$ to $3,400 \mathrm{MHz}$. We tested 10 randomly generated virtual networks for each CPU rate, and plotted the average results including confidence intervals with the confidence level of 90\%, in Figure 5.5. A confidence interval is 
calculated by Equation 3.22.

In this simulation setup, we have 5 virtual nodes per $\mathrm{VN}$, with the minimum of 1 mapper, 1 reducer, and 1 shuffler virtual node. The exact number is chosen randomly according to the uniform distribution. Moreover, we assume that for a mapper virtual node $\dot{v}_{n}^{k}, \frac{\hat{C}_{c}^{m}\left(\dot{v}_{n}^{k}\right)}{\hat{C}_{c}\left(\dot{v}_{n}^{k}\right)}=0.5$ and $\frac{\hat{C}_{c}^{\hat{m}}\left(\dot{v}_{n}^{k}\right)}{\hat{C}_{c}\left(\dot{v}_{n}^{k}\right)}=1$. Besides, for a reducer virtual node $\ddot{v}_{n}^{k}, \frac{\hat{C}_{c}^{m}\left(\ddot{v}_{n}^{k}\right)}{\hat{C}_{c}\left(\dot{v}_{n}^{k}\right)}=0$ and $\frac{\hat{C}_{c}^{m}\left(v_{n}^{k}\right)}{\hat{C}_{c}\left(\dot{v}_{n}^{k}\right)}=1$.

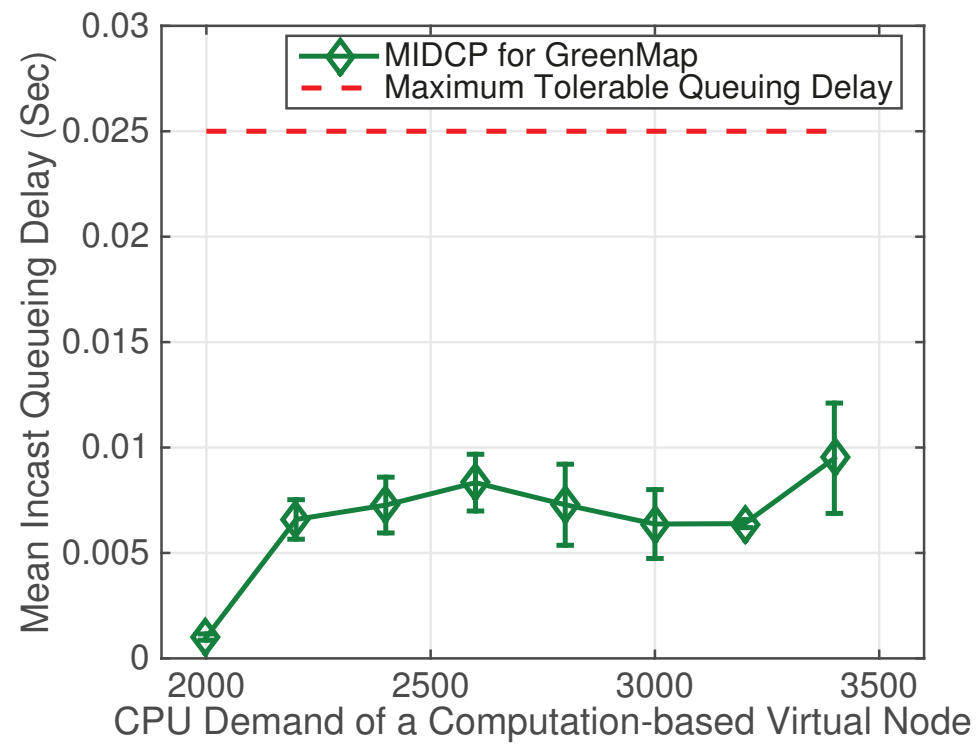

Figure 5.5: The mean incast queueing delay based on different CPU demands of a computation-based virtual node for the MIDCP, as well as the chosen maximum tolerable queueing delay $\hat{D}$ for a virtual link

According to the results in Figure 5.5, the mean incast queuing delay is always less than the determined maximum tolerable delay $\hat{D}$ of $25 \mathrm{msec}$. This confirms that the MIDCP for GreenMap could control the incast queuing delay, effectively. Nevertheless, simulation results show that the mean incast queuing delay of infinity, for any CPU demand in the range, when we relax the incast constraints in Equation 5.20. This means that for each case at least one queue over the allocated substrate path to a virtual link that terminates at an allocated reducer virtual node is unstable. So, the queue's service mean rate is less than its arrival rate. Hence, in the case we do not control the introduced incast queueing delay, the providers might not catch their latency targets for the individual MapReduce tasks. This also may result in violation of their Service Level Agreements (SLAs). 
Note that the incast queuing delay is fluctuating by changing the CPU demand. In order to satisfy the objective and the constraints, different CPU demands of a computation-based virtual node might be splitted and allocated in server substrate nodes, differently. Since the incast queuing delay is related to the assigned CPU capacity to reducer virtual nodes, it fluctuates by changing the CPU demand.

In the same simulation setup, we also measured the total consumed power by servers, switches/routes, and physical links. The results are shown in Figure 5.6. The figure shows that increasing the demanded CPU rate of computation-based virtual nodes, increases the total power consumption. This is because, we need to allocate more processing/bandwidth capacities in the data center network, in order to handle the higher demands.

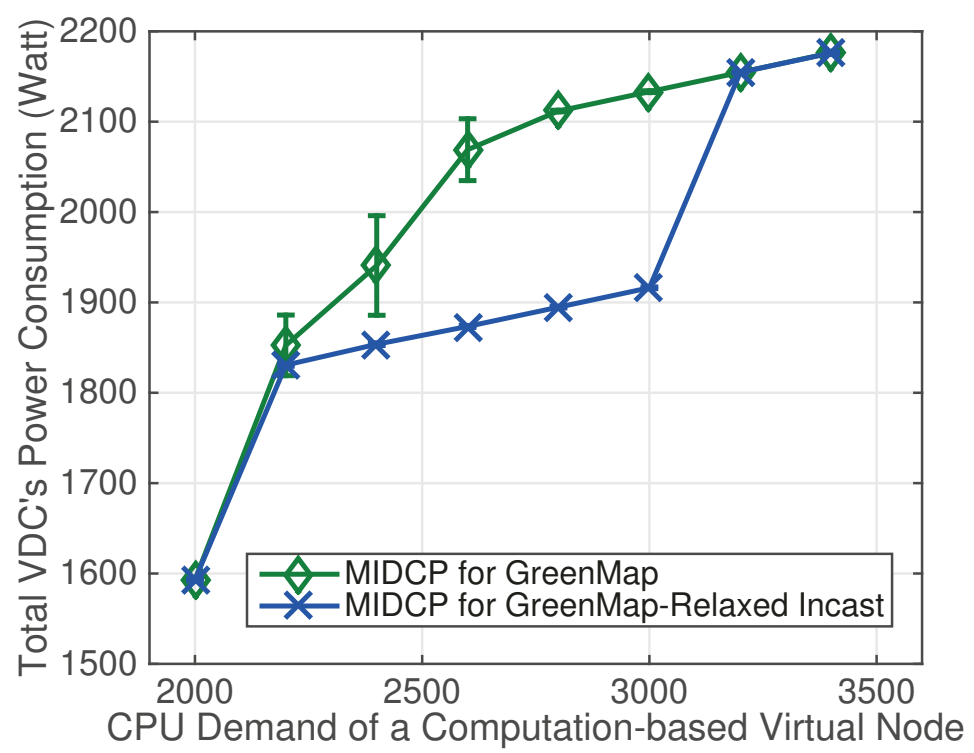

Figure 5.6: The total VDC's power consumption based on different CPU demands of a computation-based virtual node for the MIDCP, and the MIDCP when the incast constraints are relaxed

But, the interesting point about the results in Figure 5.6 is that for some demanded CPU rates, the total power consumption is higher when we control the incast queuing delay. In other words, sometimes we could save more power if we relax the incast constraints. According to Section 5.4, the way we split and map the CPU demands of reducer virtual nodes onto server substrate nodes directly impacts the incast queueing delay. Hence, when we limit the the incast queueing delay by setting the maximum tolerable queueing delay for a virtual link, we are not able to use every available 
processing/bandwidth capacity in the network to save energy. For example, we might have to turn an inactive server on to handle a CPU demand and keep the incast queueing delay in the requested range. However, if we we do not concern about the incast queueing delay, then we may be able to split the CPU demand into smaller blocks and allocate them in active servers to save the energy.

This issue does not happen when we have the enough processing capacity in active servers, so splitting the CPU demands does not violate the incast constraint. Besides, the total power consumption in the case of relaxed incast constraints might change linearly for a range of CPU demands, as we could split and allocate them in active servers without turing on inactive servers.

\subsubsection{The Heuristic}

The simulation results in Figure 5.4 confirmed that the heuristic reduces the total server power consumption of the small scale VDC, effectively. In order to verify effectiveness of the heuristic regarding saving energy in large scale data centers, we tested the heuristic as well as the state-of-the-art algorithm on a large random simulation setup, for different numbers of virtual nodes per VN. The number of virtual nodes per VN is ranged from 12 to 18 . Here, a VN has 10 shufflers, and at least 1 mapper and 1 reducer virtual node. The exact number of mapper and reducer virtual nodes are chosen randomly based on the uniform distribution. The CPU demand of a computation-based virtual node is a random value between $500 \mathrm{MHz}$ and $1,500 \mathrm{MHz}$, following the uniform distribution. Note that this range is chosen, so the state-of-the-art algorithm is able to map a computation-based virtual node onto a single server substrate node. We examined 10 randomly generated virtual networks for each number of virtual nodes per VN, and plotted the average results including confidence intervals with the confidence level of 90\%, in Figure 5.7. A confidence interval is calculated by Equation 3.22.

The results in Figure 5.7 prove that the heuristic for GreenMap significantly reduces the large scale VDC's total power consumption in comparison to the state-ofthe-art algorithm. Besides, the results show that the heuristic for GreenMap saves considerably larger amounts of power in the large random simulation setup in comparison to the small simulation setup in Figure 5.4. This means GreenMap saves the energy in large network sizes more effectively than small network sizes. This is because a larger number of computation-based virtual nodes could be splitted and 
mapped and/or collocated in servers. Besides, a larger number of shuffler virtual nodes could be collocated in switches/routers. This decreases the number of active substrate elements remarkably, in comparison to the state-of-the-art algorithm that maps each virtual node onto a single substrate node.

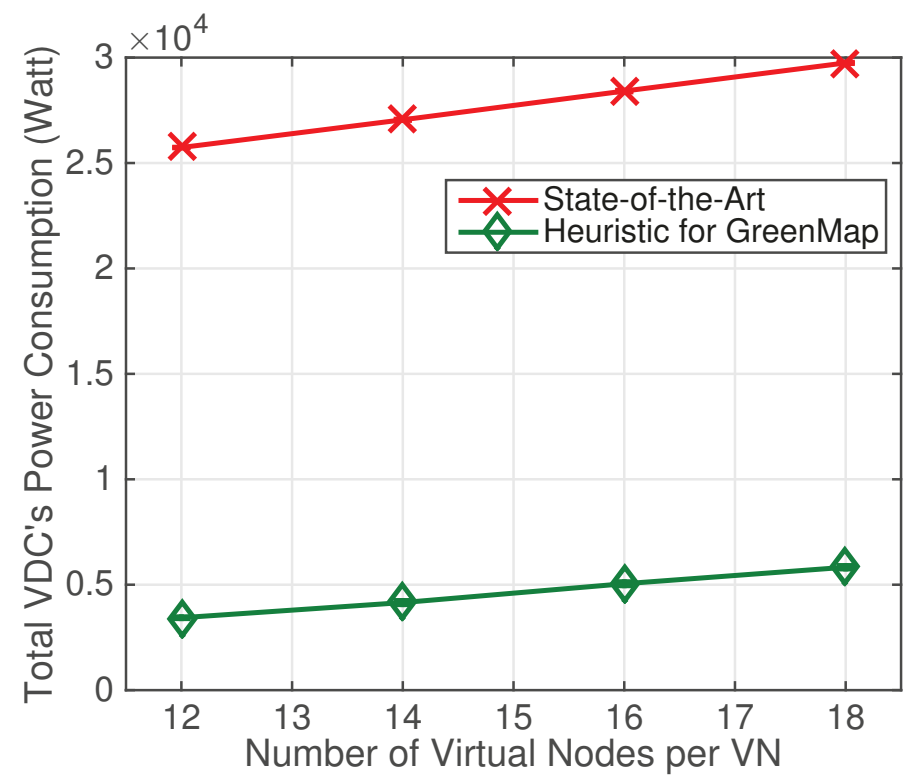

Figure 5.7: The total VDC's power consumption based on different numbers of virtual nodes per VN for the state-of-the-art algorithm, and the heuristic for GreenMap

Furthermore, we examined the heuristic on a large random simulation setup, and measured the mean incast queuing delay in the allocated substrate paths to virtual links that terminate at a reducer virtual node. This measurement is performed for different numbers of virtual nodes per VN. The number of virtual nodes per VN is ranged from 12 to 28 . A VN has 10 shufflers, and at least 1 mapper and 1 reducer virtual node. The exact number of mapper and reducer virtual nodes are chosen randomly based on the uniform distribution. The CPU demand of a computationbased virtual node is chosen randomly between $2,000 \mathrm{Mhz}$ and $3,400 \mathrm{MHz}$, according to the uniform distribution. We tested 10 randomly generated virtual networks for each number of virtual nodes per $\mathrm{VN}$, and plotted the average results including confidence intervals with the confidence level of 90\%, in Figure 5.8. A confidence interval is calculated by Equation 3.22.

Figure 5.8 confirms that the heuristic effectively controls the mean incast queuing delay, and it is always less than the defined maximum tolerable queuing delay of 
50msec. Note that the mean incast queueing delay is fluctuating by changing the number of virtual nodes per VN. This is because the algorithm might split and map the computation-based virtual nodes differently, in order to satisfy the constraints. Because the incast queuing delay is related to the assigned CPU capacity to reducer virtual nodes, it fluctuates by changing the number of virtual nodes per VN.

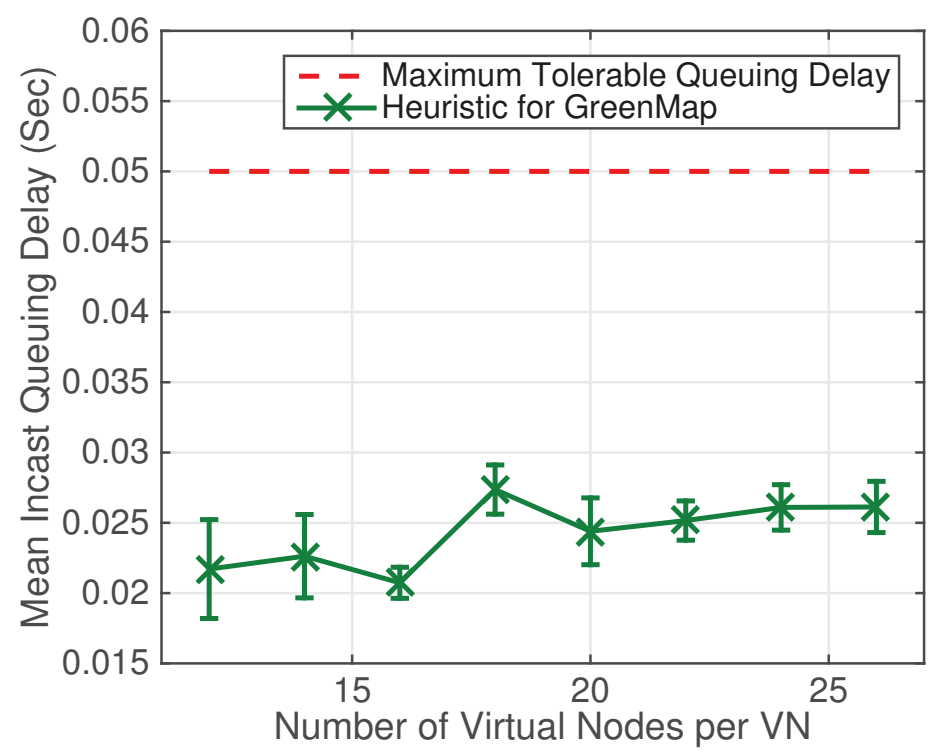

Figure 5.8: The mean inast queuing delay based on different numbers of virtual nodes per $\mathrm{VN}$ for the heuristic for GreenMap, as well as the maximum tolerable queuing delay $\hat{D}$ for a virtual link

Moreover, in another large random simulation setup, we tested the admittance ratio of the network for different values of $\hat{D}$. The admittance or acceptance ratio is the number of accepted and mapped VNs, divided by the total number of received VN requests. We considered the range of $15 \mathrm{msec}$ to $25 \mathrm{msec}$ for $\hat{D}$. We examined 10 randomly generated scenarios. In each scenario, the network receives 10 randomly generated virtual networks for every defined $\hat{D}$. Here, a virtual network has 10 shuffler virtual nodes, 5 mapper virtual nodes, and 5 reducer virtual nodes. The CPU demand of a computation-based virtual node is chosen randomly between $2,000 \mathrm{MHz}$ and $3,400 \mathrm{MHz}$, according to the uniform distribution. The average results including confidence intervals with the confidence level of 90\%, is plotted in Figure 5.9. A confidence interval is calculated by Equation 3.22.

Figure 5.9 demonstrates that the mean acceptance ratio is increasing by increasing the value of $\hat{D}$. The smaller values of $\hat{D}$ enforce the heuristic to split the processing 
demands of reducer virtual nodes in relevantly larger processing blocks, and map them onto servers. Allocating larger processing capacities in servers leaves smaller available processing capacities in them for new $\mathrm{VN}$ requests. Therefore, it is more probable that the heuristic could not map a new VN onto the data center network successfully, for smaller values of $\hat{D}$. This results in a lower network's acceptance ratio for smaller values of $\hat{D}$.

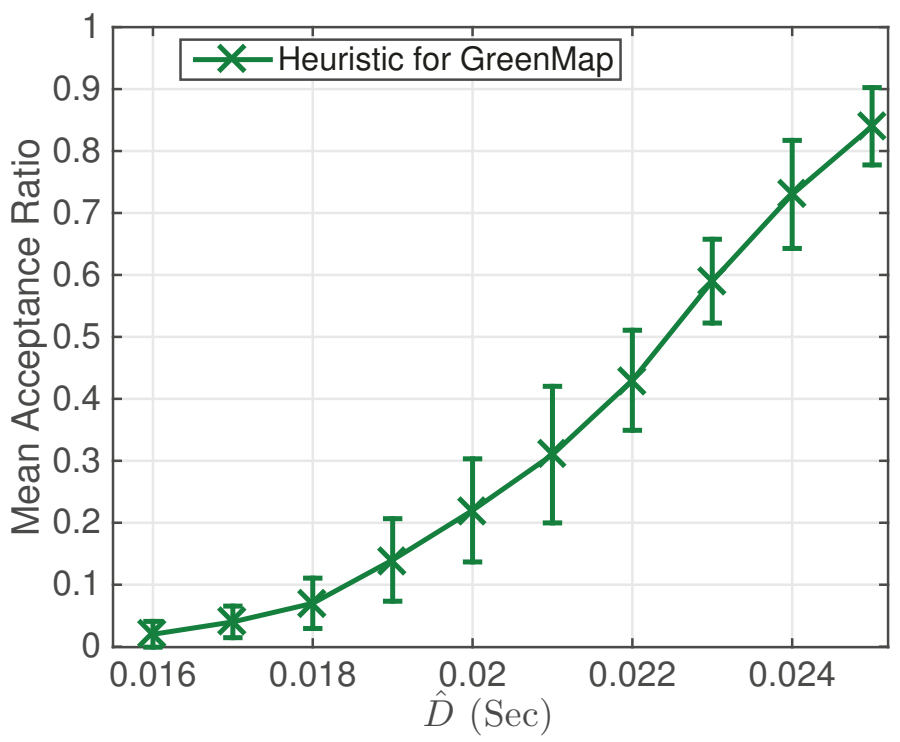

Figure 5.9: The mean acceptance ratio based on different values of $\hat{D}$ for the heuristic for GreenMap

In the same simulation setup, for when $\hat{D}$ is $50 \mathrm{msec}$, we probed the acceptance ratio for different mean traffic rates of a mapper virtual node. The results are shown in Figure 5.10. We assume that the same mean traffic rate for all mapper virtual nodes. The mean traffic rate of a mapper virtual node is ranged from $5 \mathrm{Mbps}$ to $25 \mathrm{Mbps}$. According to Equation 5.23, incrementing the mean traffic rate of mapper virtual nodes and therefore increasing $\lambda_{n}^{\mathcal{M}}$, increases the minimum amount of processing capacity the heuristic must allocate to a reducer virtual node to control the incast queuing delay. This causes smaller available processing capacities in servers for new $\mathrm{VNs}$, and accordingly reduces the acceptance ratio. 


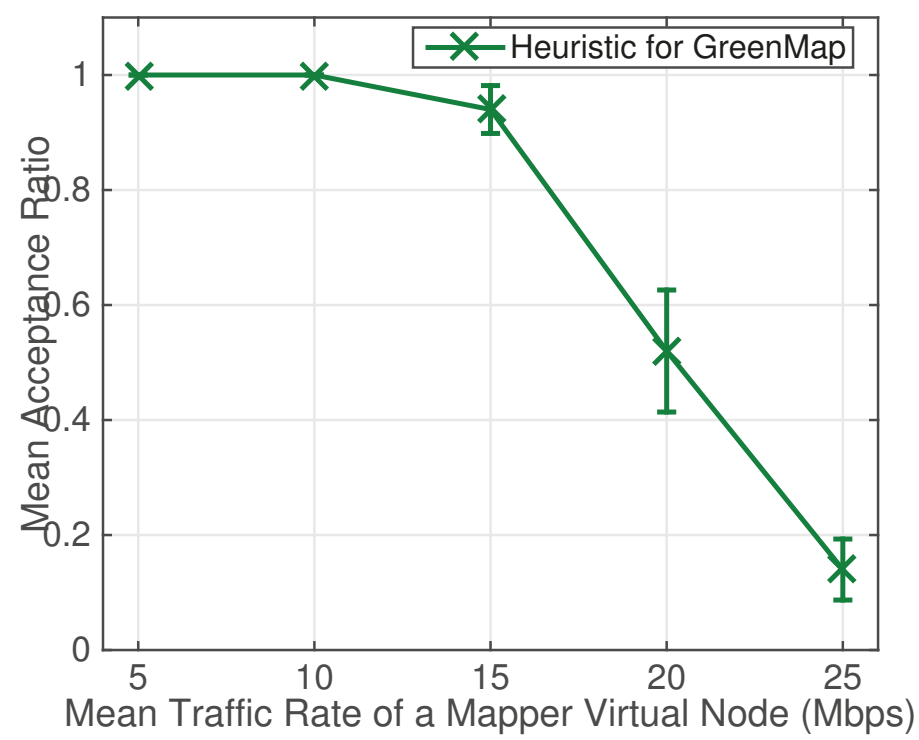

Figure 5.10: The mean acceptance ratio based on different mean traffic rates of a mapper virtual node for the heuristic for GreenMap

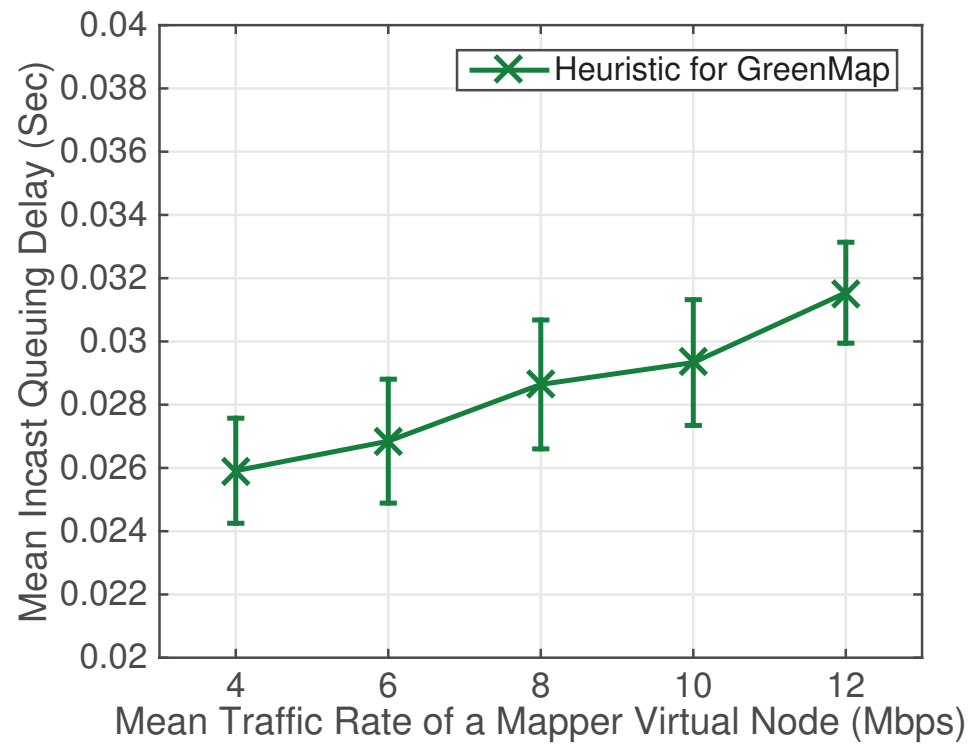

Figure 5.11: The mean incast queuing delay based on different mean traffic rates of a mapper virtual node for the heuristic for GreenMap

Moreover, in the previous simulation setup, we also measured the mean incast queuing delay in the allocated substrate paths to the virtual links that terminate at a reducer virtual node. The results are demonstrated in Figure 5.11. Different 
from the previous simulation setup, in this case we considered the range of $4 \mathrm{Mbps}$ to $12 \mathrm{Mbps}$ for the mean traffic rate of a mapper virtual node. This range is chosen, so no $\mathrm{VN}$ is rejected. As the results confirm, increasing the mean traffic rate of mapper virtual nodes, increases the mean incast queueing delay. This is because the difference between the mean service rate and the mean traffic rate in an allocated traffic capacity to the virtual links in substrate links is decreased. Therefore, according to $M / M / 1$ queue, the mean incast queuing delay is increased.

Note that every simulation setup is quite large to cover a substantial number of random virtual networks in order to verify the effectiveness of the proposed solutions. Besides, the calculated confidence intervals confirm that the results are precise enough to reveal the significances of GreenMap.

\subsection{Summary}

Saving the energy in today's data centers is a key challenge. On the other hand, data centers are moving toward virtualized data centers. In this chapter, we proposed GreenMap, a novel energy-efficient embedding method that maps heterogeneous MapReduce-based virtual networks onto a heterogeneous data center network. Moreover, for the first time, we introduced a new incast problem that specially may happen in VDCs. GreenMap also controls the incast queueing delay. An MIDCP is formulated and a novel heuristic is suggested for GreenMap. Simulation results prove that both of the solutions for GreenMap could map the heterogeneous MapReducebased VNs onto the heterogeneous data center network, and reduce a VDC's total consumed energy, effectively. It is also confirmed that both of the MIDCP and the heuristic for GreenMap control the introduced incast queueing delay. 


\section{Chapter 6}

\section{Conclusion and Future Works}

Energy consumption in ICT is a large portion of the total energy consumed in industrial countries, while is rapidly increasing. VNEs have recently emerged as a solution in this technology, to address the challenges of the future Internet. VNEs also play a fundamental role toward virtualizing data centers. Consequently, it is essential to develop novel techniques to reduce a VNE's energy consumption. In this thesis, we have defined and solved two main problems regarding the energy-saving in a VNE.

\subsection{Off-Peak Energy Optimization for a VNE}

First, we discussed a problem in Chapter 3 that optimizes the total energy consumption in a VNE during the off-peak time. We approached this problem in two sub-problems that increase in the complexity. Increasing the problem's complexity provides higher levels of energy-saving.

Due to simpler technical implementation and high potential of energy-saving in a network's links, the first sub-problem is restricted to energy-saving for links in a VNE. In this regard, we discussed multiple novel energy-saving solutions that globally/locally optimize a VNE's link energy consumption, during the off-peak time. A coarse-grained global reconfiguration, which reconfigures the mapping of every virtual link, is formulated to provide the benchmark results. Besides, a fine-grained local reconfiguration is also proposed. It reconfigures the mapping for some of the already mapped virtual links, according to the defined stress rates. This method enables the providers to adjust the level of the reconfiguration. So, they accordingly can control the possible traffic disruptions due to the reconfiguration.

An ILP is formulated for each solution, according to two power models. Since 
the ILPs are $\mathcal{N} \mathcal{P}$-hard, a novel heuristic algorithm is also suggested. Different from the previous research studies, our method does not decrease a network's admittance ratio for new virtual networks. Besides, our solution is not limited to a sub-topology, and so it has a larger degree of freedom to save the energy. In addition, we discussed how differently we should approach the problem in the case of non-splittable traffic in comparison to splittable traffic, to have a wide enough search zone for the re-mapping.

We examined the formulated solutions through extensive simulations, and investigated the impacts of different factors on their energy-saving ability. Simulation results reveal that the energy-saving solutions are noticeably effective and the heuristic achieves closely to the optimum points.

Because physical nodes are also essential energy consumers, we extended the first sub-problem and developed energy-saving solutions for nodes and links in a VNE during the off-peak period in Chapter 4. In the second sub-problem, first, we defined an energy-saving solution for a VNE that minimizes its node and link energy consumption during the off-peak time, by reconfiguring the mapping for some of both virtual nodes and links. Because reconfiguring the mapping for both of virtual nodes and virtual links may be expensive or cause the interruptions to the normal network operations, we came up with another methodology that minimizes the intermediate nodes and links' energy consumption in a VNE, during the off-peak time, by reconfiguring the mapping for only some of the virtual links.

An ILP is defined for each method, according to two power models, and considering the impact of traffic splittability. These methods enable the providers to change the level of the reconfiguration by adjusting the stress rate's threshold. Therefore, they can control the possible traffic interruptions of the reconfiguration. Clearly, there is a trade-off between energy-saving level and the possible traffic interruptions. This is investigated through simulations. Similar to the first sub-problem, this method does not decrease a network's admittance ratio for new virtual networks. As the ILPs are $\mathcal{N} \mathcal{P}$-hard, we also suggested a heuristic algorithm for off-peak node and link energy optimization by local link reconfiguration.

These proposed energy-saving methods are examined by extensive simulations. They prove the heuristic works closely to the optimum points set by the optimization program.

Note that when it is only possible to save the energy in physical links, and we do not want to sleep any physical node unless we sleep all its adjacent virtual links, we 
have to use the proposed solutions in Chapter 3. In this case, we can not use the proposed solutions in Chapter 4, as they save the energy of physical links if and only if they save the energy in physical nodes by setting them into the sleep mode.

The developed energy-saving heuristics can be easily implemented in Softwaredefined Networking (SDN) controllers to optimize the network's energy consumption during the off-peak time.

In regards to the first problem, there are some open areas for the future works. First, the proposed solutions assume that the off-peak periods and the associated demands are known. In this regard, it is probable to develop new specific methods that dynamically determine the off-peak time and demands in VNEs. Combining our energy-saving solutions with such methods provides an effective and stand-alone energy-saving strategy for VNEs. Second, it is beneficial to consider the time space in the simulations and use the published real networks' traffic rates for different time periods to investigate the effectiveness of the solutions regarding saving the energy over a period of time. Third, collocating multiple virtual nodes of a VN in a single substrate node has been recently suggested. Therefore, it is possible to extend our solutions to consider the virtual node collocation to save more energy. Fourth, the proposed heuristics are designed based on a fixed power model. It is valuable to design new heuristics that can employ any power model.

\subsection{GreenMap}

In the second problem, we proposed GreenMap in Chapter 5, a novel energy-efficient embedding method that maps heterogeneous MapReduce-based virtual networks onto a heterogeneous data center network. Different from any existing studies, GreenMap makes it probable to split and map computation-based virtual nodes onto a data center network. Accordingly, it enables the providers to embed computation-based VNs onto a data center network. Besides, it handles the heterogeneity of MapReducebased VNs and the data center network.

For the first time, we introduced a new incast problem for virtualized data centers. Therefore, a novel approach is demonstrated that controls the introduced incast queueing delay. We tackled the incast problem during the provisioning process. So, it prevents the incast problem to happen at the first point. This is a much more efficient approach in comparison to the existing solutions for incast that try to recover the 
connection after that incast happens. The problem is formulated as a MIDCP. Since it is $\mathcal{N} \mathcal{P}$-hard, a novel and scalable heuristic is also proposed for the problem that could achieve closely to the optimum points.

We examined both the MIDCP and the heuristic through extensive simulations, and checked the impacts of different factors. It is demonstrated that how controlling the incast queueing delay may affect the energy-saving level and a network's admittance ratio.

In regards to the second problem, there are some open areas for the future works. First, GreenMap could be extended to a more general solution, so it will be able to map not only a MapReduce-base virtual network, but also any computation-based virtual network. Second, the employed queueing model of $\mathrm{M} / \mathrm{M} / 1$ could estimate the average end-to-end queuing delay. However, as a future work, the more precise queuing models like MMPP/M/1 are required to be employed in order to find the exact end-to-end queuing delay. In this regard, the challenge of finding an end-toend queueing delay based on the employed queuing model will emerge. Applying a more precise queuing model may help to save more energy. Third, it is valuable to consider the finite size queue models and derive the respective energy-saving solutions. Fourth, the proposed heuristic could be modified, so it will be able to employ any power model. 


\section{List of References}

[1] O. Gemikonakli, E. Ever, and E. Gemikonakli, "Performance modelling of virtualized servers," in 12th International Conference on Computer Modelling and Simulation (UKSim), IEEE, pp. 434-438, Cambridge, 24-26 March 2010.

[2] G.-Q. Zhang, G.-Q. Zhang, Q.-F. Yang, S.-Q. Cheng, and T. Zhou, "Evolution of the internet and its cores," New Journal of Physics, IOP Publishing, vol. 10, no. 12 , p. $123027,2008$.

[3] R. Bolla, F. Davoli, R. Bruschi, K. Christensen, F. Cucchietti, and S. Singh, "The potential impact of green technologies in next-generation wireline networks: Is there room for energy saving optimization?," Communications Magazine, IEEE, vol. 49, no. 8, pp. 80-86, 2011.

[4] R. Bolla, R. Bruschi, F. Davoli, and F. Cucchietti, "Energy efficiency in the future internet: a survey of existing approaches and trends in energy-aware fixed network infrastructures," Communications Surveys 83 Tutorials, IEEE, vol. 13, no. 2, pp. 223-244, 2011.

[5] S. Ricciardi, D. Careglio, G. Santos-Boada, J. Sole-Pareta, U. Fiore, and F. Palmieri, "Saving energy in data center infrastructures," in First International Conference on Data Compression, Communications and Processing (CCP), IEEE, pp. 265-270, Palinuro, 21-24 June 2011.

[6] E. C. D. INFSO, "Impacts of information and communication technologies on energy efficiency," Tech. Rep. September, 2008.

[7] D. T. Group, "Ecological indicators," tech. rep., 2008. http://www.crbericht.telekom.com/site08/en/daten-fakten/kennzahlen/oekologischekennzahlen-/index.php.

[8] B. T. Group, "Sustainability report," tech. rep., 2009. http://www.btplc.com/Societyandenvironment/Ourapproach/ Sustainabilityreport.

[9] ITWales, "Green evangelist to call for big changes in computer use to aid environment," 2007. http://www.itwales.com/997539.htm.

[10] A. Beloglazov, R. Buyya, Y. C. Lee, and A. Zomaya, "A taxonomy and survey of energy-efficient data centers and cloud computing systems," Advances in Computers, Academic Press, vol. 82, no. 2, pp. 47-111, 2011. 
[11] S. Ricciardi, D. Careglio, G. Santos-Boada, J. Sol-Pareta, U. Fiore, and F. Palmieri, "Saving energy in data center infrastructures," in First International Conference on Data Compression, Communications and Processing (CCP), IEEE, pp. 265-270, Palinuro, 21-24 June 2011.

[12] R. Brown, "Report to congress on server and data center energy efficiency: Public law 109-431," Lawrence Berkeley National Laboratory, 2008.

[13] J. Hamilton, "Cooperative expendable micro-slice servers (cems): low cost, low power servers for internet-scale services," in Conference on Innovative Data Systems Research (CIDR), ACM, Pacific Grove, CA, 4-7 January 2009.

[14] J. S. Turner and D. E. Taylor, "Diversifying the internet," in Global Telecommunications Conference (GLOBECOM), IEEE, vol. 2, pp. 6 pp.-760, St. Louis, MO, 2-2 December 2005.

[15] N. Chowdhury and R. Boutaba, "A survey of network virtualization," Computer Networks, Elsevier, vol. 54, no. 5, pp. 862-876, 2010.

[16] M. F. Zhani, Q. Zhang, G. Simon, and R. Boutaba, "Vdc planner: Dynamic migration-aware virtual data center embedding for clouds," in International Symposium on Integrated Network Management (IM), IFIP/IEEE, pp. 18-25, Ghent, 27-31 May 2013.

[17] J. F. Botero and X. Hesselbach, "Greener networking in a network virtualization environment," Computer Networks, Elsevier, vol. 57, no. 9, pp. 2021-2039, 2013.

[18] T. Anderson, L. Peterson, S. Shenker, and J. Turner, "Overcoming the internet impasse through virtualization, ieee," Computer, vol. 38, no. 4, pp. 34-41, 2005.

[19] P. D. Standard, "802.1 q/d10, ieee standards for local and metropolitan area networks: Virtual bridged local area networks," tech. rep., IEEE, 1997.

[20] D. McDysan and M. Carugi, "Service requirements for layer 3 provider provisioned virtual private networks (ppvpns)," tech. rep., Nortel Networks, 2005.

[21] R. Callon and M. Suzuki, "A framework for layer 3 provider-provisioned virtual private networks (ppvpns)," tech. rep., RFC 4110, 2005.

[22] L. Andersson and E. Rosen, "Framework for layer 2 virtual private networks (12vpns)," tech. rep., RFC 4664, 2006.

[23] W. Augustyn and Y. Serbest, "Service requirements for layer 2 providerprovisioned virtual private networks," The Internet Society, 2006.

[24] A. T. Campbell, H. G. D. Meer, M. E. Kounavis, K. Miki, J. B. Vicente, and D. Villela, "A survey of programmable networks," Computer Communication Review, ACM SIGCOMM, vol. 29, no. 2, pp. 7-23, 1999.

[25] N. Feamster, L. Gao, and J. Rexford, "How to lease the internet in your spare time, acm," SIGCOMM Computer Communication Review, vol. 37, no. 1, pp. 61-64, 2007. 
[26] N. M. K. Chowdhury and R. Boutaba, "Network virtualization: state of the art and research challenges," Communications Magazine, IEEE, vol. 47, no. 7, pp. 20-26, 2009.

[27] I. Houidi, W. Louati, D. Zeghlache, and S. Baucke, "Virtual resource description and clustering for virtual network discovery," in International Conference on Communications Workshops (ICC Workshops), IEEE, pp. 1-6, Dresden, 14-18 June 2009.

[28] I. Houidi, W. Louati, and D. Zeghlache, "A distributed virtual network mapping algorithm," in International Conference on Communications (ICC), IEEE, pp. 5634-5640, Beijing, 19-23 May 2008.

[29] M. Yu, Y. Yi, J. Rexford, and M. Chiang, "Rethinking virtual network embedding: substrate support for path splitting and migration," Computer Communication Review, ACM, vol. 38, no. 2, pp. 17-29, 2008.

[30] I. Houidi, W. Louati, W. B. Ameur, and D. Zeghlache, "Virtual network provisioning across multiple substrate networks," Computer Networks, Elsevier, vol. 55, no. 4, pp. 1011-1023, 2011.

[31] J. Lu and J. Turner, "Efficient mapping of virtual networks onto a shared substrate," tech. rep., Washington University in St.Louis, 2006.

[32] Y. Zhu and M. H. Ammar, "Algorithms for assigning substrate network resources to virtual network components.," in INFOCOM, IEEE, pp. 1-12, Barcelona, April 2006.

[33] I. Houidi, W. Louati, D. Zeghlache, P. Papadimitriou, and L. Mathy, "Adaptive virtual network provisioning," in Workshop on Virtualized infrastructure systems and architectures, ACM, pp. 41-48, New Delhi, 30 August-3 September 2010 .

[34] J. He, R. Zhang-Shen, Y. Li, C.-Y. Lee, J. Rexford, and M. Chiang, "Davinci: dynamically adaptive virtual networks for a customized internet," in CoNEXT, $A C M$, p. 15, Madrid, December 2008.

[35] M. V. Luzgachev and K. E. Samouylov, "The resource allocation problem in the design of virtual private networks with unicast and multicast connections," in International Congress on Ultra Modern Telecommunications and Control Systems and Workshops (ICUMT), IEEE, pp. 1096-1101, Moscow, 18-20 October 2010 .

[36] S. Peng, R. Nejabati, E. Escalona, D. Simeonidou, M. Anastasopoulos, K. Georgakilas, A. Tzanakaki, and A. Vernitski, "Performance modelling and analysis of dynamic virtual optical network composition," in 16th International Conference on Optical Network Design and Modeling (ONDM), IEEE, pp. 1-5, Colchester, 17-20 April 2012.

[37] J. Fan and M. H. Ammar, "Dynamic topology configuration in service overlay networks: A study of reconfiguration policies.," in INFOCOM, IEEE, Barcelona, 23-29 April 2006. 
[38] N. M. K. Chowdhury, M. R. Rahman, and R. Boutaba, "Virtual network embedding with coordinated node and link mapping," in INFOCOM, IEEE, pp. 783791, Rio de Janeiro, 19-25 April 2009.

[39] U. Javed, M. Suchara, J. He, and J. Rexford, "Multipath protocol for delaysensitive traffic," in Communication Systems and Networks and Workshops (COMSNETS), IEEE, pp. 1-8, Bangalore, 5-10 January 2009.

[40] G. N. Higginbottom, Performance evaluation of communication networks. Artech House, Inc., 1998.

[41] Q. Duan, "Modeling and analysis for end-to-end service performance in virtualization-based next generation internet," in Global Telecommunications Conference (GLOBECOM), IEEE, pp. 1-6, Miami, FL, 6-10 December 2010.

[42] Q. Duan, "End to end modelling and performance analysis for network virtualisation in the next generation internet," International Journal of Communication Networks and Distributed Systems, Inderscience, vol. 8, no. 1, pp. 53-69, 2012 .

[43] J.-Y. L. Boudec and P. Thiran, Network calculus: a theory of deterministic queuing systems for the internet, vol. 2050. Springer, 2001.

[44] Y. Wei, J. Wang, and C. Wang, "A traffic prediction based bandwidth management algorithm of a future internet architecture," in 3rd International Conference on Intelligent Networks and Intelligent Systems (ICINIS), IEEE, pp. 560563, Shenyang, 1-3 November 2010.

[45] Y. Wei, J. Wang, C. Wang, and C. Wang, "Bandwidth allocation in virtual network based on traffic prediction," in International Conference on Computer Design and Applications (ICCDA), IEEE, vol. 5, pp. V5-304-V5-307, Qinhuangdao, 25-27 June 2010.

[46] M. Tsugawa and J. A. Fortes, "Characterizing user-level network virtualization: performance, overheads and limits," International Journal of Network Management, Wiley Online Library, vol. 20, no. 3, pp. 149-166, 2010.

[47] R. Gad, M. Kappes, R. Mueller-Bady, and I. Ritter, "Network performance in virtualized environments," in 17th International Conference on Networks (ICON), IEEE, pp. 275-280, Singapore, 14-16 December 2011.

[48] Y. Zhu, Y. Zhang, C. Ying, and W. Lu, "Queuing model based end-to-end performance evaluation for mpls virtual private networks," in International Symposium on Integrated Network Management (IM), IEEE, pp. 482-488, Long Island, NY, 1-5 June 2009.

[49] P. Barham, B. Dragovic, K. Fraser, S. Hand, T. Harris, A. Ho, R. Neugebauer, I. Pratt, and A. Warfield, "Xen and the art of virtualization, acm," SIGOPS Operating Systems Review, vol. 37, no. 5, pp. 164-177, 2003.

[50] N. Bobroff, A. Kochut, and K. Beaty, "Dynamic placement of virtual machines for managing sla violations," in International Symposium on Integrated Network Management (IM), IEEE, pp. 119-128, Munich, 21-25 May 2007. 
[51] R. J. Figueiredo, P. A. Dinda, and J. A. Fortes, "A case for grid computing on virtual machines," in International Conference on Distributed Computing Systems, IEEE, pp. 550-559, Providence, Rhode Island, 19-22 May 2003.

[52] G. Khanna, K. Beaty, G. Kar, and A. Kochut, "Application performance management in virtualized server environments," in Network Operations and Management Symposium (NOMS), IEEE, pp. 373-381, Vancouver, BC, 3-7 April 2006.

[53] C. P. Sapuntzakis, R. Chandra, B. Pfaff, J. Chow, M. S. Lam, and M. Rosenblum, "Optimizing the migration of virtual computers, acm," SIGOPS Operating Systems Review, vol. 36, no. SI, pp. 377-390, 2002.

[54] M. Steinder, I. Whalley, D. Carrera, I. Gaweda, and D. Chess, "Server virtualization in autonomic management of heterogeneous workloads," in International Symposium on Integrated Network Management (IM), IEEE, pp. 139-148, Munich, 21-25 May 2007.

[55] C. A. Waldspurger, "Memory resource management in vmware esx server, acm," SIGOPS Operating Systems Review, vol. 36, no. SI, pp. 181-194, 2002.

[56] A. P. Bianzino, C. Chaudet, F. Larroca, D. Rossi, and J. Rougier, "Energyaware routing: a reality check," in GLOBECOM Workshops (GC Wkshps), IEEE, pp. 1422-1427, Miami, FL, 6-10 December 2010.

[57] R. Tucker, J. Baliga, R. Ayre, K. Hinton, and W. Sorin, "Energy consumption in ip networks," in Optical Communications,, p. 1, Brussels, 21-25 September 2008.

[58] X. Fan, W.-D. Weber, and L. A. Barroso, "Power provisioning for a warehousesized computer," in ACM SIGARCH Computer Architecture News, ACM, vol. 35, pp. 13-23, 2007.

[59] D. Economou, S. Rivoire, C. Kozyrakis, and P. Ranganathan, "Full-system power analysis and modeling for server environments," in Workshop on Modeling Benchmarking and Simulation (MOBS), pp. 13-23, Boston, MA 17-21 June 2006 .

[60] A. Fischer, M. T. Beck, and H. D. Meer, "An approach to energy-efficient virtual network embeddings," in International Symposium on Integrated Network Management (IM), IFIP/IEEE, pp. 1142-1147, Ghent, 27-31 May 2013.

[61] J. Chabarek, J. Sommers, P. Barford, C. Estan, D. Tsiang, and S. Wright, "Power awareness in network design and routing," in The 27th Conference on Computer Communications (INFOCOM), IEEE, pp. 457-465, Phoenix, AZ, 13-18 April 2008.

[62] B. Wang, X. Chang, J. Liu, and J. K. Muppala, "Reducing power consumption in embedding virtual infrastructures," in Globecom Workshops (GC Wkshps), IEEE, pp. 714-718, Anaheim, CA, 3-7 December 2012. 
[63] Z. Zhang, S. Su, X. Niu, J. Ma, X. Cheng, and K. Shuang, "Minimizing electricity cost in geographical virtual network embedding," in Global Communications Conference (GLOBECOM), IEEE, pp. 2609-2614, Anaheim, CA, 3-7 December 2012 .

[64] S. Su, Z. Zhang, X. Cheng, Y. Wang, Y. Luo, and J. Wang, "Energy-aware virtual network embedding through consolidation," in Computer Communications Workshops (INFOCOM WKSHPS), IEEE, pp. 127-132, Orlando, FL, 25-30 March 2012.

[65] C. Gunaratne, K. Christensen, and B. Nordman, "Managing energy consumption costs in desktop pcs and lan switches with proxying, split tcp connections, and scaling of link speed," International Journal of Network Management, Wiley Online Library, vol. 15, no. 5, pp. 297-310, 2005.

[66] R. Hays, A. Wertheimer, and E. Mann, "Active/idle toggling with low-power idle," in IEEE802.3az Task Force Group Meeting, 2008.

[67] C. Gunaratne, K. Christensen, and S. W. Suen, "Ngl02-2: Ethernet adaptive link rate (alr): Analysis of a buffer threshold policy," in Global Telecommunications Conference (GLOBECOM), IEEE, pp. 1-6, San Francisco, CA, 27 November-1 December 2006.

[68] S. Antonakopoulos, S. Fortune, and L. Zhang, "Power-aware routing with rateadaptive network elements," in GLOBECOM Workshops (GC Wkshps), IEEE, pp. 1428-1432, Miami, FL, 6-10 December 2010.

[69] J. F. Botero, X. Hesselbach, M. Duelli, D. Schlosser, A. Fischer, and H. D. Meer, "Energy efficient virtual network embedding," Communications Letters, IEEE, vol. 16, no. 5, pp. 756-759, 2012.

[70] E. Ghazisaeedi, N. Wang, and R. Tafazolli, "Link sleeping optimization for green virtual network infrastructures," in Globecom Workshops (GC Wkshps), IEEE, pp. 842-846, Anaheim, CA, 3-7 December 2012.

[71] D. Lo, L. Cheng, R. Govindaraju, L. A. Barroso, and C. Kozyrakis, "Towards energy proportionality for large-scale latency-critical workloads," in 41st annual international symposium on Computer architecuture, IEEE, pp. 301-312, Minneapolis, MN, 14-18 June 2014.

[72] C. Xin, B. Wang, X. Cao, and J. Li, "Logical topology design for dynamic traffic grooming in wdm optical networks," Journal of Lightwave Technology, IEEE, vol. 24, no. 6, p. 2267, 2006.

[73] L. Chiaraviglio, M. Mellia, and F. Neri, "Reducing power consumption in backbone networks," in International Conference on Communications (ICC), IEEE, pp. 1-6, Dresden, 14-18 June 2009.

[74] G. Shen and R. Tucker, "Energy-minimized design for ip over wdm networks," Journal of Optical Communications and Networking, IEEE/OSA, vol. 1, no. 1, pp. 176-186, 2009. 
[75] L. Chiaraviglio, M. Mellia, and F. Neri, "Minimizing isp network energy cost: formulation and solutions," Transactions on Networking (TON), IEEE/ACM, vol. 20, no. 2, pp. 463-476, 2012.

[76] Y. Zhang, M. Tornatore, P. Chowdhury, and B. Mukherjee, "Energy optimization in ip-over-wdm networks," Optical Switching and Networking, Elsevier, vol. 8, no. 3, pp. 171-180, 2011.

[77] F. Idzikowski, S. Orlowski, C. Raack, H. Woesner, and A. Wolisz, "Dynamic routing at different layers in ip-over-wdm networks-maximizing energy savings," Optical Switching and Networking, Elsevier, vol. 8, no. 3, pp. 181-200, 2011.

[78] G. Rizzelli, A. Morea, M. Tornatore, and O. Rival, "Energy efficient trafficaware design of on-off multi-layer translucent optical networks," Computer Networks, Elsevier, vol. 56, no. 10, pp. 2443-2455, 2012.

[79] H. Feng and Y. Shu, "Study on network traffic prediction techniques," in International Conference on Wireless Communications, Networking and Mobile Computing, IEEE, pp. 1041-1044, Wuhan, 23-26 September 2005.

[80] A. S. San-Qi, "A predictability analysis of network traffic," Computer Networks, Elsevier, vol. 39, no. 4, pp. 329-345, 2002.

[81] P. Mahadevan, P. Sharma, S. Banerjee, and P. Ranganathan, A power benchmarking framework for network devices, pp. 795-808. NETWORKING, Springer, 2009.

[82] C. Gunaratne, K. Christensen, and B. Nordman, "Managing energy consumption costs in desktop pcs and lan switches with proxying, split tcp connections, and scaling of link speed," International Journal of Network Management, Wiley Online Library, vol. 15, no. 5, pp. 297-310, 2005.

[83] S. Even, A. Itai, and A. Shamir, "On the complexity of time table and multicommodity flow problems," in 16th Annual Symposium on Foundations of Computer Science, IEEE, pp. 184-193, USA, 13-15 October 1975.

[84] B. M. Waxman, "Routing of multipoint connections," Selected Areas in Communications, IEEE, vol. 6, no. 9, pp. 1617-1622, 1988.

[85] A. Fischer, J. F. B. Vega, M. Duelli, D. Schlosser, X. H. Serra, and H. D. Meer, "Alevin-a framework to develop, compare, and analyze virtual network embedding algorithms," Open-Access-Journal Electronic Communications of the EASST, 2011.

[86] J. Zhu and T. Wolf, "Vnmbench: a benchmark for virtual network mapping algorithms," in 21st International Conference on Computer Communications and Networks (ICCCN), IEEE, pp. 1-8, Munich, 30 July-2 August 2012.

[87] M. T. Beck, A. Fischer, H. de Meer, J. F. Botero, and X. Hesselbach, "A distributed, parallel, and generic virtual network embedding framework," in International Conference on Communications (ICC), IEEE, pp. 3471-3475, 2013. 
[88] E. D. Andersen and K. D. Andersen, The MOSEK interior point optimizer for linear programming: an implementation of the homogeneous algorithm, pp. 197232. High performance optimization, Springer, 2000.

[89] "Geant; the pan european data network project." http://www.geant.net/.

[90] J. Bisschop, AIMMS-optimization modeling. ParagonDecisionTechnology, 2006.

[91] J. Y. Yen, "Finding the k shortest loopless paths in a network," management Science, vol. 17, no. 11, pp. 712-716, 1971.

[92] M. Grant and S. Boyd, "Cvx: Matlab software for disciplined convex programming, version 2.0 beta," September 2013. http://cvxr.com/cvx.

[93] J. Baliga, R. W. Ayre, K. Hinton, and R. Tucker, "Green cloud computing: Balancing energy in processing, storage, and transport," Proceedings of the IEEE, IEEE, vol. 99, no. 1, pp. 149-167, 2011.

[94] L. A. Barroso and U. Hlzle, "The case for energy-proportional computing," Computer, IEEE, vol. 40, no. 12, pp. 33-37, 2007.

[95] J. Dean and S. Ghemawat, "Mapreduce: Simplified data processing on large clusters," in 6th Symposium on Operating Systems Design Implementation, $A C M$, p. 10, San Francisco, CA, 5 December 2004.

[96] J. Dean and S. Ghemawat, "Mapreduce: simplified data processing on large clusters," Communications, ACM, vol. 51, no. 1, pp. 107-113, 2008.

[97] W. Li, H. Yang, Z. Luan, and D. Qian, "Energy prediction for mapreduce workloads," in Ninth International Conference on Dependable, Autonomic and Secure Computing (DASC), IEEE, pp. 443-448, Sydney, NSW, 12-14 December 2011.

[98] C. Guo, G. Lu, H. J. Wang, S. Yang, C. Kong, P. Sun, W. Wu, and Y. Zhang, "Secondnet: a data center network virtualization architecture with bandwidth guarantees," in 6th International Conference on emerging Networking EXperiments and Technologies (CoNEXT), ACM, p. 15, Philadelphia, PN, 30 November-3 December 2010.

[99] M. Chowdhury, M. R. Rahman, and R. Boutaba, "Vineyard: Virtual network embedding algorithms with coordinated node and link mapping," Transactions on Networking (TON), IEEE/ACM, vol. 20, no. 1, pp. 206-219, 2012.

[100] D. Nagle, D. Serenyi, and A. Matthews, "The panasas activescale storage cluster: Delivering scalable high bandwidth storage," in Conference on Supercomputing, ACM/IEEE, p. 53, 06-12 November 2004.

[101] J. Hwang, J. Yoo, and N. Choi, "Deadline and incast aware tcp for cloud data center networks," Computer Networks, Elsevier, vol. 68, pp. 20-34, 2014.

[102] M. Alizadeh, A. Greenberg, D. A. Maltz, J. Padhye, P. Patel, B. Prabhakar, S. Sengupta, and M. Sridharan, "Data center tcp (dctcp)," Computer Communication Review, ACM SIGCOMM, vol. 41, no. 4, pp. 63-74, 2011. 
[103] S. Kandula, S. Sengupta, A. Greenberg, P. Patel, and R. Chaiken, "The nature of data center traffic: measurements and analysis," in 9th conference on Internet measurement conference, ACM SIGCOMM, pp. 202-208, Chicago, IL, 4-6 November 2009.

[104] H. Wu, Z. Feng, C. Guo, and Y. Zhang, "Ictcp: incast congestion control for tcp in data-center networks," Transactions on Networking (TON), IEEE/ACM, vol. 21, no. 2, pp. 345-358, 2013.

[105] Y. Ren, Y. Zhao, P. Liu, K. Dou, and J. Li, "A survey on tcp incast in data center networks," International Journal of Communication Systems, Wiley Online Library, vol. 27, no. 8, pp. 1160-1172, 2014.

[106] A. Phanishayee, E. Krevat, V. Vasudevan, D. G. Andersen, G. R. Ganger, G. A. Gibson, and S. Seshan, "Measurement and analysis of tcp throughput collapse in cluster-based storage systems.," in Conference on File and Storage Technologies, ACM, vol. 8, pp. 1-14, San Jose, CA, 26-29, February 2008.

[107] E. Krevat, V. Vasudevan, A. Phanishayee, D. G. Andersen, G. R. Ganger, G. A. Gibson, and S. Seshan, "On application-level approaches to avoiding tcp throughput collapse in cluster-based storage systems," in 2nd international workshop on Petascale data storage: held in conjunction with Supercomputing'07, ACM, pp. 1-4, Reno, NV, 11 November 2007.

[108] Y. Chen, R. Griffith, J. Liu, R. H. Katz, and A. D. Joseph, "Understanding tcp incast throughput collapse in datacenter networks," in The 1st workshop on research on enterprise networking, ACM, pp. 73-82, Barcelona, 17-21 August 2009 .

[109] M. Grant, S. Boyd, and Y. Ye, Disciplined convex programming. Springer, 2006.

[110] H. Aljazzar and S. Leue, "K: A heuristic search algorithm for finding the $\mathrm{k}$ shortest paths," Artificial Intelligence, Elsevier, vol. 175, no. 18, pp. 2129-2154, 2011 .

[111] J. Hershberger, M. Maxel, and S. Suri, "Finding the k shortest simple paths: A new algorithm and its implementation," Transactions on Algorithms (TALG), $A C M$, vol. 3, no. 4, p. 45, 2007.

[112] S. Ricciardi, D. Careglio, U. Fiore, F. Palmieri, G. Santos-Boada, and J. SolPareta, "Analyzing local strategies for energy-efficient networking," in NETWORKING 2011 Workshops, IFIP, pp. 291-300, Springer, Valencia, 9-13 May 2011. 